Aleksey Nikolsky ${ }^{1}$ and Antonio Benítez-Burraco ${ }^{2}$

Authors' affiliations

1. Independent Researcher, Austin, TX, United States

2. Department of Spanish Language, Linguistics and Literary Theory (Linguistics), Faculty of Philology, University of Seville, Spain

Corresponding authors:

Aleksey Nikolsky (orcid.org/0000-0001-5572-9438): alekseynikolsky@ gmail.com

Antonio Benítez Burraco (orcid.org/0000-0003-4574-5666): abenitez8@us.es

\title{
Human aggression and music evolution: a model
}

\section{Introduction}

2. What is music?

2.1. Existing problems in defining music

2.2. A formal definition of music

2.3. Delving into the structural features of music

2.4. The functional typology of music

2.5. The dependencies between music functions

3. The effects of cultural transmission on music structures

3.1. The overview of the recent experimental research

3.2. Cultural bias in oral transmission of music

3.3. The role of formats of transmission in genesis of music idioms

4. The evolution of music: an overview of the latest literature

5. "Personal song": the missing link between the animal calls and human music

6. Human aggression and music evolution: some concerns

6.1. Aggression management through the course of human evolution

6.2. Possible causes for the reduction in reactive aggression: focusing on the environment and hospitality

6.3. The origins of hospitality and its relation to kinship

6.4. Kinship and personal song

6.5. The contribution of prosocial behavior to music complexity

7. A model of music evolution

7.1. Stage 0: Musilanguage

7.2. Stage 1: Proto-music

7.3. Stage 2: Timbre-oriented music

7.4. Stage 3: Pitch-oriented music

7.5. Intonational typology of music: from personal to collective

7.6. Stage 4: Tonality of collective music 


\section{Introduction}

In this paper we present a model of the genesis and evolution of music under the effects of changes in the management of aggression throughout the course of human cultural evolution. The connection between a general decline in reactive aggression ${ }^{1}$ and increased cooperativeness that underlies advances in sophisticated technology and culture was established in the so-called "selfdomestication" hypothesis of human evolution [1]. However, present-day humans also exhibit relatively high levels of proactive aggression. ${ }^{2}$ Here, we argue that the progressive decline of reactive aggression and the progressive increase of proactive aggression might have paved the way, through diverse effects on human interactions and social features, to the evolution of music. We hypothesize its course, starting with the hypothetical emergence of proto-music from musilanguage [2] as a system of communicating information, related to one's emotional state. In the shortest, our model presents the following account of the evolutionary development:

Once formed, proto-music supported continuous increase in differentiation between the coordinated personal and private uses of music-like structures, on the one hand, versus their uncoordinated collective use (as in musilanguage), on the other hand. The former obtained predominantly monophonic organization and acquired a diverse typology of signals within a very few basic genres, while relying on the anthropophonic intonation (see Section 7.5). ${ }^{3} \mathrm{At}$ this point, the reduction of reactive aggression stayed limited to the family circle.

Thereafter, monophonic organization evolved into monodic (i.e., supporting basic rhythmic accompaniment), while introducing the naturophonic intonation (Section 7.5). ${ }^{4}$ The preferred timbre-classes (musical equivalents of linguistic phonemes) were combined into sets, forming musical modes. This stage was accompanied by the substantial reduction in reactive aggression within the entire community and the genesis of proto-kinship. The latter probably brought to life an important new function of music - marking an individual with a unique musical pattern to reflect their social identity within a group (analogous to the so-called "personal song", as found amongst many indigenous ethnicities of the Extreme North).

The next major development was the invention and exploration of musical instruments, which generated a new type of musical intonation - organophonic. ${ }^{5}$ The latter introduced the pitch

\footnotetext{
${ }^{1}$ Impulsive aggression, typically resulting from the experience of fear or anger, as a retaliation to a perceived provocation or hostile intent.

2 Premeditated, goal-directed aggression, usually designed to achieve an objective beyond physical violence, motivated by the projected benefits.

${ }^{3}$ A manner of connecting 2 different pitch levels that is determined by the internal anatomical capacities of the vocal apparatus rather than an intentional attempt to produce a sound with specific sonic characteristics.

${ }^{4} \mathrm{~A}$ manner of connecting 2 different pitch levels that is determined by an intention to imitate the properties of some environmental objects, external to the signaler, akin to onomatopoeia.

${ }^{5}$ Organophonic (from organology - the study of musical instruments) intonation usually connects 2 pitch levels in a manner peculiar to a specific musical instrument, adopting that instrument as a model for musicking on other
} 
reference for vocal musicking and directed music evolution towards conversion from timbral to pitch orientation: at first in the form of ekmelic ${ }^{6}$ and thereafter emmelic intonations. ${ }^{7}$ This advance enabled the use of coordinated musical ensembles, probably family-based, promoting the development of kinship, and the emergence of hospitality codes. During this period, the overall levels of aggression must have reached their absolute minimum throughout human evolution.

The final stage was marked by the introduction of metrophonic intonation, ${ }^{8}$ which defined a set of pitch- and interval-classes to be used in a music composition. This reform radically transformed music by replacing the diversity of musical modes with a few standardized musical keys, operable by grammar-like rules and requiring multi-level subordinations to a single pitch-class - instituting the so-called tonality. Its genesis was accompanied by the emergence of ethos - a doctrine of the supposed psycho-emotional power of specific musical keys to influence individuals and society at large. The long-term outcome of cultivation of ethos was consolidation and politicization of society and rise of nationalism, which triggered the increasing growth of proactive aggression and escalation of warfare.

We start this paper by identifying the most important obstacles for studying the evolution of music (Section-2). To overcome them, we formulate a structural definition of music and identify 11 aspects of music expression and 7 structural features that distinguish music from oral language. We argue that without such formal distinctions no objective discussion of matters, related to music and musicality, is possible. Any inquiry into the evolution of music, in addition, requires an insight into functions of music. We break the most common functions of music into 14 operational types and demonstrate that they form pairs of dependencies, so that one function cannot operate without another function being already active. To establish the primordial (non-dependent) function, we order all the operational types according to their dependencies and end up with the progression that closely resembles the ontogenetic pattern of music acquisition in early childhood. We then extend this pattern towards adulthood, following the Vygotskian approach to sociogenesis [3,4], and establish the parallels between ontogenetic and phylogenetic patterns of development of music skills in light of the corresponding uses of music. This allows us to distinguish between 10 developmental phases, starting from the instinctive vocal production of music-like signals (similar to animal communication) and ending with the deliberate use of convention-based musical idioms (similar to idiomatic expressions of language). All 10 phases rely on the primordial function of hedonistic stimulation and the use of music as a "learning aid". The centrality of the capacity to enjoy sounds per se and become motivated by this enjoyment to explore and satisfy one's curiosity suggests that the entire evolution of music and its divergence from language can and should be explained by the development of hedonistic and learning functionality in higher order functions.

\footnotetext{
instruments, as well as for vocal music. Some organophonic models might generate sliding pitch, and therefore relate approximate pitch-levels rather than definite pitch-classes.

${ }^{6}$ Ekmelic (from Greek 'unmelodic', aka, unclear, not suitable for tunes) intonation is based on continuous frequency modulations and scalable melodic intervals, indefinite in pitch, inferred through reference to a particular position within a melodic contour and/or the range of a vocal or instrumental register.

7 Emmelic (from Greek 'melodic', aka, suitable for tunes) intonation is based on discrete changes in frequency in accordance with the set of selected pitch-classes, separated by relatively fixed melodic intervals that usually slightly vary in size depending on the melodic context to increase the melodic tension or relaxation.

${ }^{8}$ Metrophonic (from Greek metron - 'measure') intonation connects 2 well-defined pitch-classes according to a uniform rational principle, so that the distance between them can be expressed in some metric units.
} 
In the next section, we examine how transmission of music influences its structural features - a fundamental matter for the evolution of music. Biologically determined constraints on perception and processing of auditory signals shape structural patterns at every instance of their reproduction by different participants in a transmission act, depending on the number of participants. The entire evolutionary line of 10 developmental phases clearly displays the tendency of growing participation: from solitary and duetting at the earliest phases to the entire community at the last phases. This increase in numerosity interacts with 4 formats of music transmission and 14 operational types in a very complex way. We show how their interaction results in the emergence of a musical idiom through the chain of "music acts," gradually expanding from personal to collective. We integrate the findings of the experimental research on transmission of music (and language) with the ethnomusicological data and demonstrate that the increase of the number of participants in long transmission chains necessarily introduces cultural bias and tends to change the balance between transmitter and receiver from the emphasis on transmittance (favoring expression) to the emphasis on reception (favoring learnability). The end result of the entire chain comes as a kind of "natural selection," through the series of successful "adaptations" to new uses with new participants, and "extinctions" of those variants that failed to do so. This process is accompanied by the growing complexity of the social structure of "music actors": from a "motherchild" dyad to a conglomerate of thousands of people. What underlies this line of development is the relation of trust and care, modeled upon the affection between mother and child and projected over larger and larger groups of unrelated people. This is what we believe is the driving force that determines the evolution of both music and language - what we call "conspecific sympathy."

In Section-4, we review current theories about the origins of music. After briefly characterizing competing views, we focus on two principal approaches: music as a credible signal and music as a social bond. We argue that although their proponents see them as irreconcilable, in reality, they just focus on different stages in the evolution of music, and therefore fail to encompass its entirety (including all developmental phases). We support our view with ample evidence, mostly from present-day hunter-gatherer groups that live in ecological conditions similar to those during the Quaternary glaciation in Europe and from the tonal organization of the earliest forms of nonimitative spontaneous musical vocalizations, produced by human infants during their first 2-3 years of life. We demonstrate that the currently dominant view that music can be defined as a tool of social cohesion due to music's supposed inherent collectivism is applicable only to the later stages of music evolution. When it comes to the origins of music, it was not that the invention of music made humans social, but to the contrary, the emergence of prosociality enabled transformation of animal-like musilanguage into human-specific proto-music and thereafter - into music.

We see the missing link between animal communication and human music in the institute of the so-called "personal song," surviving amongst those indigenous populations of northeastern Eurasia, North America, and Greenland that retain lifestyles very similar to those of Eurasian populations of the late Paleolithic (Section-5). Personal song is the cultural tradition of assigning a specific melodic structure to each individual of an indigenous community as means of personal identification. Like one's passport, a personal song usually represents one's personality, age, geographic place of origin, and ancestral lineage - by means of re-configuring melodic, rhythmic, and timbral patterns taken from the ancestral songs. The "honest" use of personal song is protected

\footnotetext{
${ }^{9}$ We conceive the notion of "music act" by the analogy to "speech act" - as the totality of information transmitted by the performer(s) of music via all available channels of communication, including the circumstances of the music performance and the previous history of similar performances.
} 
in indigenous societies by religious taboos on unauthorized reproduction of someone else's song, punishable by the retribution of its owner (and his family), as well as the danger of public condemnation. As a result, personal song is used as lingua franca between neighboring Siberian ethnicities, instrumental for their custom of marrying into a different ethnicity/clan. This ties personal song to cooperation and friendliness, while supporting territoriality and ancestrality, similar to animal "songs," making personal song a likely prototype for a transitory stage between animal-like proto-music and music.

We dedicate Section-6 to investigating the possible origins of prosociality. To those causes that have been already identified, we add the deterioration of climate in those paleo-ecosystems that still remained inhabitable, thereby posing the pressure for human inhabitants (and possibly, some pre-human hominins) to cooperate in order to increase mutual chances for survival. The choice of this strategy in favor of fighting for scarce available resources was probably ideologically fixed (on some religious basis) - as observed in numerous hospitality codes, still existing across a huge area, from the Caucasus to Alaska. Their distribution was even wider in antiquity, suggesting that these codes constitute a prehistoric epi-cultural phenomenon, probably determined by geo-climatic factors. The ethnographic research on hospitality, especially amongst the surviving hunter-gatherer societies, provides an insight into a possible motivation behind the strategy to befriend a stranger despite chances of his aggression. The dialectics of hospitality-hostility underlie the emergence of hospitality and vendetta codes, both based on the opposition of "us" versus "them" along the kinship axis. We look into the possible birth time of kinship and tie it to the rise of personal song. The latter can be dated to the Paleo-Siberian population crossing Beringia, when it was accessible for land travel, and to the earliest signs of human presence in the Americas. The amazing conservancy of personal song, observed by ethnographers during the last 250 years amongst peoples of the Extreme North, its wide distribution (from the Kola peninsula to the Midwest US), supported by genetic data, and its organic relation to ancestry that remains central to the belief system of its users (the Ancestor Cult) - all suggest that prehistoric populations that migrated to America carried personal song with them rather than acquired it via cultural contacts over the Bering Strait later.

The available archeological evidence demonstrates the existence of an uninterrupted cultural tradition across most of Siberia and the Russian Far East at least from the Neolithic to the late 18th century (the time of first ethnographic records). We argue that the Altai-Sayan region was the most likely place, where the genesis of kinship, personal song, and cross-cultural hospitality took place. Once set in place, personal song probably triggered the ongoing feedback between the further development of social structures that, in turn, influenced music structures through the practice of musicking within a social unit (e.g., couples of parents and/or children, family, extended family, clan, etc.). Here, human attachments found correspondence in patterns of attaching timbre- and/or pitch-classes to generate musical modes, used by these participants in their musicking. In the same vein, the growth in complexity of social organization must have promoted the complexity of tonal organization - materialized in conversion of timbral modes into, at first, modes of indefinite pitch, then, modes of definite pitch, followed by the numerical growth of pitch-classes (from monotonal to multitonal modes, see Section 6.5). This development most certainly led to the emergence of hierarchical tonal organization in tetratonic modes, giving life to music syntax (analogous to the development of syntax in esoteric languages).

In Section-7, we lay out our own model of co-evolution of music and language in response to the influence of changes in aggression management: from their undifferentiated state in animal-like 
musilanguage, followed by their differentiation into human proto-language and proto-music, resulting in their divergence and subsequent autonomous development. We link this line of evolutionary development to the genesis and evolution of kinship and growing complexity of social structural units - starting from basic dyadic relations, supported by means of mostly personal and private communication, and ending with the nexus of relations in complex structured societies, supported by collective communication. Instrumental in this transformative interaction between social organization, on the one hand, and tonal organization of music along with syntactic organization of language, on the other hand, comes the evolution of intonational typology, which we outline, following Asafyev's theory of musical intonation [5-8].

In Section-8, we summarize the content of this entire paper. In final Section-9, we list possible ways of verification of our hypothesis and suggest directions for future research.

\section{What is music?}

The task of investigating the evolutionary origins of music requires first to agree on what constitutes "music" and distinguishes it from animal calls as well as from human language.

\subsection{Existing problems in defining music}

Much of the controversy in the research literature on evolution of music stems from the misunderstanding of the term "music": more often than not, authors talk about different things while using the same word. In the worst scenario, instead of providing a clear-cut general definition of music as the starting point of a discussion, papers merely list scores of features as to how different people have been viewing music - and then prompt the reader to use his intuition, as though the reader, supposedly like everyone else, already knows what music is. Structural definitions (i.e., how music-like sounds are typically arranged) and functional definitions (i.e., how people commonly use music-like sounds in their life) are often intermingled as if they are equivalent. But they are not. And overall, structural definitions tend to be either over-generalized to the extent of not excluding phenomena that are regarded non-musical by cultural conventions, in practice, or overly restrictive and Eurocentric, limiting the concept of music to the conventions of Western tonality alone (according to textbooks of Western classical music).

For instance, Mehr et al. [9] and Savage et al. [10] argue against each other's accounts of the evolution of music. However, both studies look into quite different cultural paradigms of music and adopt different perspectives of looking at them. The former defines music structurally: as "an auditory display built from melodies and rhythms". The latter defines music functionally - it claims that the conglomerate of the principal ways in which people use music-like sounds would suffice to distinguish music from non-music. Both definitions fall short of providing reliable criteria for identifying music.

Mehr et al. pick wrong structural criteria: neither melodies nor rhythms distinguish music from non-music. There are forms of music without melody (e.g., African talking drums or Kumi-daiko in Japan) and without rhythm (e.g., most known forms of plainchant and many forms of recitative). Even more importantly, there are artifacts that possess melodic contours and rhythm, which however, are not considered music by their creators and listeners. For instance, poetry in tone 
languages, such as Thai chanthalak is characterized by contrasts in melodic contour and rhythm but their organization differs from those in Thai traditional music [11]. In Western classical music, the ovation after a recital performance is not considered music in spite of the clapping of the audience being metrically regular due to the entrainment effect and the "bravo" shouts reproducing the same prosodic contour and thus possessing an inherent melodic shape that is reproduced by each shouter.

Savage et al. [10] emphasize the presence of discrete pitches and rhythmic proportions as the principal structural criteria of music. However, this definition disqualifies those forms of music that are based on continuous changes in pitch, indefinite pitch, and timbre-oriented musicking [12] as well as recitative-like genres and styles of music. On the other hand, under this view, a Western vocalist warming up by doing a rhythmisized solmization exercise "Mi Me Ma Mo Mu" in scales or arpeggios, is in fact, making music. The truth of the matter is that neither vocalists, nor their audiences consider such exercises to be a music work. Rather, they think that such warmups are not intended to express anything and are therefore unworthy of paying attention to. And people do not have to witness the performance of such exercise in order to come to this conclusion - hearing its audio recording would suffice, thereby entailing that music can be effectively distinguished from non-music purely by interpreting the auditioned music structures.

The "mechanistic" approach (based on the functional interpretation of how the music is used), which Savage et al. advocate to resolve controversies in identifying different types of music, relies on "musical behaviors" rather than musical structures. The problem with this approach is that "musical behaviors" apart from being quite deceptive (as highlighted in the example of solmization above) constitute a circular argument. In order to identify a behavior as "musical" one must already know what "music" is, so identification of music by means of analyzing a musical behavior is simply impossible. Moreover, as it has become recently common in Western ethnomusicology (many papers on music do not contain a single analysis of a musical text), study of musical behaviors focuses not on music structures but on people who believe or are believed to practice music. But what if such assumptions are wrong? ${ }^{10}$ This makes attempts to identify the presence of music patterns by means of musical behaviors alone quite arbitrary, questioning the validity of conclusions derived from such an approach. To draw a parallel, how would linguists react if someone tried to conduct a study of a language by taking into consideration only "speaking behaviors" and their functions, disregarding the analysis of phonetic and syntactic structures?

A comparison with what we observe in the domain of language is illuminating. Parallels between language evolution and music evolution are well-known [see 14,15 for review]. Although humans show a propensity for acquiring and using languages in order to fulfill many goals, linguists carefully distinguish between the human faculty of language (e.g., language-readiness or human linguisticality), the languages we speak (their suite of distinctive structural features as well as their commonalities with other languages), and the diverse uses of language. In our view, the starting point for any attempt to delineate the evolutionary history of music should be a comprehensive and reliable structural definition of music, followed by a comprehensive and reliable

\footnotetext{
10 In many indigenous societies, vocalizations associated with magic beliefs are not regarded as "music" per se, despite their close similarity (to the extent of being audibly undistinguishable) to the sound of genres that are regarded as music: e.g., animal spells, animal lullabies, children lullabies, and milking songs [13]. Similar confusion characterizes the use of recitative in liturgical service of a number of religious denominations. Yet another example is the blurry distinction between "rapping" and "singing", observed by many rappers and their fans. An example of "music" that has nothing to do with musical behaviors is the widespread use of Aeolian harps and wind-chimes across the globe from antiquity until modern day.
} 
characterization of musical behaviors, with both aspects ultimately pointing to the human generalized propensity to learn and use music (aka musicality). Thus, defining "musicality" does not make sense without defining "music" first.

\subsection{A formal definition of music}

In the next sections we will adhere to the following structural definition of music:

Music is such tonal organization of multiple aspects of expression (the 5 most important of which are: pitch, rhythm, meter, dynamics, and timbre) that entrains listeners and performers and transposes the performers' intentions to emotionally stir listeners through vocal and/or instrumental performance.

The concept of "tonal organization" entails the presence of salient changes in the temporal unveiling of a specific "aspect of expression." The latter can be defined as a coherent and continuous manner of channeling information within a selected substrate of acoustic properties of sound. For example, the aspect of melody relates successions of tones in frequency, whereas rhythm - in duration. According to the data of comparative ethnomusicology and psychoacoustics, music has at least 11 aspects that are used cross-culturally for expression and find equivalents in animal communication [13]. We shall briefly define all 11 aspects:

1. Melody (aka pitch contour, often qualified as the "horizontal" aspect of music) - changes of the fundamental frequency between the consecutive tones;

2. Harmony (often qualified as the "vertical" aspect) - changes in the configuration of those harmonics that are shared between the harmonic series of simultaneously sounding tones;

3. Texture (sometimes called the "depth" aspect, since it concerns the number of voices and parts) - a scheme of arranging consecutive and contemporaneous relations between all tones engaged in a music work or its sizeable section (e.g., homophony vs. polyphony);

4. Thematicity (aka music form, since it concerns the number of themes per music work) coherence and consistency of the salient features of pitch/time organization of music (aka "theme") by means of which one can identify a music work (e.g., monothematic vs. bi-thematic song that consists of verse and chorus);

5. Tempo - an overall impression of pace and character of a music movement (slow vs. fast);

6. Rhythm - changes in relative duration of the consecutive tones (short vs. long);

7. Meter - a scheme of periodic grouping of beats extracted from the music movement through the interaction of patterns of pitch and rhythm in a specific tempo (e.g., binary vs. ternary);

8. Articulation - a manner of attaching and/or detaching those tones, dyads, and chords that are grouped together (e.g., legato vs. staccato);

9. Dynamics - changes of amplitude between the consecutive or simultaneous tones, when one tone is perceived as deliberately louder or softer than another;

10. Register - breaking of the entire frequency range of a music work (aka "ambitus") or its part in multipart textures (aka "tessitura") into zones of timbrally homogenous pitch levels (e.g., light vs. dark registers);

11. Timbral quality - sustained spectral characteristics of successions of tones, dyads and chords, which can form contrasting relations in successions between different types of tonal coloration or their simultaneous combination (e.g., nasal, thin, sharp, fluffy, hard). 
Pitch contour, rhythm/meter, and dynamics constitute the most common aspects of music expression, so that the absence of structural changes in any of them (e.g., a strict monotone, a metronomic rhythm, or a perfectly flat dynamic level of the onsets of tones) should be taken as an indication that the auditory material under consideration is not music.

On the other hand, the presence of discernable patterns in tonal organization of each of 5 principal aspects of expression (pitch, rhythm, meter, dynamics, and timbre) can be safely interpreted as an indication that the auditory material constitutes music.

In order for the auditory stimuli to be considered music, sounds arranged in particular ways along the axes of 11 expression aspects must have the capacity to make a listener undergo psychophysiological changes in synchrony with changes in the corresponding aspects of expression. The simplest case is the connection between a detected structural pattern in music and a specific affective state - which can be direct or indirect and passive or active:

- Passive direct connection. The listener hears that the melody is created in a minor key and realizes that the music expresses sadness.

- Passive indirect connection. The listener hears that the music contains many patterns of dotted rhythm in fast tempo, which creates the impression of ongoing bouncing, which in turn, is associated with excitement and fun.

- Active direct connection. The music-maker feels sad or wants to project a sad expression through music and generates a melody in a minor key.

- Active indirect connection. The music-maker feels bubbly and frolicky or wants to project such expression and chooses the ongoing dotted rhythmic patterns to generate the fastbouncing music movement.

What is crucial here - every instance of detecting a conventional meaningful pattern (what we call "music idiom") triggers the realization of a corresponding psycho-physiological state. This realization can be immediately felt, if the connection between a pattern and an affective state has been culturally primed (minor key and sadness) or based on instinctive response (accentuation on every instance of a longer tone in a dotted rhythm causes a muscle contraction). Alternatively, the realization can be estranged from feelings: a listener or a music-maker can understand that ongoing dotted rhythm is meant to express excitement and fun, but not actually experience this state, say, because of depression. In this case, it is necessary to distinguish between an actual emotion of a music user and a "musical emotion" conveyed in music.

Even a more complex case is a situation of solitary performance, exceedingly common for numerous indigenous music traditions [12]. In Western classical music, too, musicians often perform privately, "for themselves". Even more widespread is humming or whistling specific tunes that match one's psycho-physiological state during some extra-musical activity. Professional musicians can "playback" a piece of music silently, in their mind. In all such cases, a performer constitutes a listener, generating a feedback loop: e.g., a singer hears the sound of his own voice and emotionally reacts to changes in music patterns, which in turn, fuels the expression of music. For this reason, solitary music-making and audiation still abide by the rule of correspondence between a specific structural pattern and a specific affective state.

Another crucial criterion of music is that the listener's emotional reaction must be transposable over numerous instances of performance. The same type of auditory material should evoke the 
same type of emotional reaction regardless of the exact circumstance in which the performance occurs: e.g., lullaby-like music should be calming, whether it is sung by a mother rocking her child, rendered by professional singers at the recital as an art-song, or played by an orchestra as a symphonic movement. It is this criterion that distinguishes genuine music from music-like behaviors, such as warming up before the performance or drilling arpeggios or scales. Music performance, as a rule, implies a deliberate intention (or a half-conscious orientation, for humming or whistling for oneself) to project a specific musical emotion. ${ }^{11}$

\subsection{Delving into the structural features of music}

Below we provide a detailed list of the main structural features of music. These structural differences allow us to figure out if a sound artifact constitutes music or not, but also to differentiate music from other verbal behaviors, particularly with oral language, based on their respective formal properties.

\section{Music idioms in multiple aspects of expression}

Music engages an assortment of idiomatic patterns for each of its multiple basic aspects of expression - melody, harmony, rhythm, meter, articulation, dynamics, texture, and music form of which only melody and meter find limited linguistic analogs. Below is the brief outline of 9 common aspects of musical expression:

a) Melody engages pitch- and interval-classes in combination with changes in melodic direction to produce a diverse assortment of idiomatic patterns (i.e., 2-tone intonations and motifs that usually consist of 3-6 tones and are associated with specific expressions). They characterize a particular music genre, style, and culture [17]. These patterns are semantically distinguished through musical genres (e.g., relaxing lullabies are characterized by the prevalence of melodic intervals of descending minor $3 r d$, gently falling, whereas mobilizing marches - by a leap of ascending 4th).

Such an organization finds a linguistic analog in the lexical tones of tone languages. However, lexical tones are considerably simpler than the melodic intonations - most often limited to just 2 opposites (rising or high vs. falling or low). Only occasionally lexical tones feature additional categories (up to 5). Yet another melody-like linguistic feature is prosodic intonation that characterizes sentence types (e.g., enunciative, interrogative, imperative, etc.). Compared to music, such systems are much simpler and support substantially fewer typological patterns for effective semiosis. In contrast, most common music systems of today usually employ 5-12 pitch-classes and 8-17 interval-classes, forming hundreds, if not thousands of various melodic intonations (see 6.5).

b) Harmony also engages pitch and interval classes but in a different dimension - "vertically" (i.e., contemporaneously), as opposed to the "horizontal" (i.e., successive) dimension of melody. Harmony generates chords and polyphonic counterpoints, where the configuration,

\footnotetext{
11 The inherent emotionality of music has been denied by Stravinsky and some of the later modernist and postmodernist Western composers. Their position seems to be a form of self-promotion. They evoke a "revolutionary" rhetoric to avoid market competition with previously successful composers, seeking to establish a brand new market niche, where they would be authenticated as their "pioneers". Notably, most of the composers who chose this route avoid competing within the niches established by their fellow-modernists, instead looking for ways to "invent" newer and newer experimental methods of "expression" - often disregarding whether these methods are perceptible by the concert audiences. Such an anti-conventional and anti-emotional stance violates the very biomusicological foundation of naturally formed music traditions (see [16] for the discussion).
} 
distribution, and typology of interval classes distinguishes one music system from another (e.g., Western classical homophony is based on the harmonic intervals of 3rd and 5th and triads, whereas the Georgian traditional polyphony - on the intervals of 4th and 5th and trichords, including 4th-chords).

Harmony serves as primary means to generate fluctuations in tonal tension and relaxation, where the former fuels the melodic and harmonic development that becomes terminated by the latter. Alternation of tension and relaxation generates harmonic syntax (subdivision of a flow of sounds into phrases and sentences) that superficially resembles clauses and sentences of natural languages. However, harmony does not constitute a musical universal - it evolves only in those music cultures that use multi-part textures with functional specialization of parts or their groups. Consistent use of harmony leads to the crystallization of harmonic "idioms." Common harmonic progressions obtain valence (aka affective quality) based on their gradations in tension and relaxation. Examples of harmonic idioms are: 1) sad minor triad vs. happy major triad, 2) sophisticated qualia distinctions between various types of the 7th-chords, and 3) progressions of chords, such as "resolution" (stable harmony follows unstable harmony) or "false resolution" (standard resolution is unexpectedly replaced with the more unstable harmony).

There is nothing even remotely similar to such organization in speech.

C) Rhythm engages proportional contrasts between longer and shorter sounds to produce dozens of idiomatic patterns by grouping a certain number of sounds together. The repertory of such patterns enables a listener to tell one musical culture from another (e.g., prevalence of punctured, aka "dotted" rhythms in Polish music vs. reverse punctured, aka "Scotch snap" in Scottish music). Rhythmic idioms are distinguished through the system of musical genres (e.g., the punctured figure "long-short-long", distributed over 2 beats, is associated with the expression of energy and characterizes processional genres like march or polonaise and leaping dances, such as mazurka).

Rhythm finds a very basic analog in phonemic vowel length of quantity languages. However, the latter are most often limited to just 2 opposites (long vs. short vowels) and rarely include a third intermediate length type. Rhythmic patterns can be formed by the interaction of quantity and accent (e.g., Yakut). The interaction of stressed and unstressed syllables can also generate rhythmic grouping in non-quantity languages, if they are isochronous (e.g., Spanish). However, compared to music, such systems are much simpler and usually do not generate stable patterns that contribute to semiosis. A music system usually employs 4-9 rhythmic divisions (e.g., whole-note, half-note, etc.) that can be of regular or irregular proportions, each of which can be divided into $2,3,5$, or even shorter rhythmic values. Moreover, a musical rhythmic group can contain up to 5-6 tones or even a long passage about 10-20 tones (e.g., a tirata).

Such diversity of rhythmic divisions and rhythmic values greatly exceeds the diversity of pitch classes, which explains why many music cultures based on fewer than 7 pitch classes are characterized by rhythmic complexity and heterogeneity. Many genres of music can be recognized by the presence of characteristic rhythmic figures that determine their expression (e.g., bolero, tango, waltz, mazurka, march). Rhythm of verbal speech does not support such characteristic patterning and diversity of elements.

d) Meter engages a number of sounds that become periodically grouped according to their duration (estimated by the units of so-called "beat") and the distribution of dynamic stress, 
forming a characteristic pattern of regular binary and/or ternary "pulse-classes," sustained throughout an entire music work or its sizeable portion. "Pulse-classes" can comprise complexes ("pulse-groups", e.g., $(3+3)+(2+2+2)+(3+3)+(3+3))$ in mixed or composite meters. Pulse-groups, in turn, can vary by the placement of a stress to form an anacrusis of different proportions in relation to the size of a periodic group. Most meters are defined semantically through the system of musical genres: e.g., march in general is characterized by having 2 or 4 pulse-groups of binary or ternary division with an anacrusis equal to a single beat - which is associated with the expression of strength and confidence. Thereby, genres put in place metric idioms: the presence of a metric "march idiom" in a symphonic movement will necessarily make it sound strong and confident, even if the movement is not strictly speaking a march (e.g., Tchaikovsky - 3rd movement from Symphony No.6). Often metric idioms develop their own typologies based on variations in metric characteristics. Thus, marches break into 3 metric sub-classes, each characterized by its own semantics:

- funeral, slow, and quick-step military marches, and recreational processions, e.g., pasodobles and cakewalks usually have "cut time", i.e., binary 2 beats as $2 / 4$ or $2 / 2$ "alla breve", which makes the movement appear relatively easy-going while being energetic - goal-oriented and directional due to the prominence of a single "left-right" pattern, each occurrence of which generates a new impetus;

- solemn, double and rifles marches, anthems, as well as circus marches usually feature "common time", i.e., compound binary 4 beats, which projects the impression of some effort or an enforcement due to the perceived straight-forward "inertia" implied by the grouping of two "left-right" patterns together in a single metric formula;

- two-step dances, smoother parade marches, such as Scottish pipe marches, march-like popular folk-songs, and operatic processions often use a compound ternary 6/8 meter, which projects the impression of relaxed swaying from side to side and induces the leisurely or entertaining feeling akin to social dances.

Metric "idioms" are similar to poetic feet that also can specialize in certain expressions that might suit specific poetic genres due to the employed scheme of distribution of a stress and a length of a foot. However, poetic speech is overall atypical for verbal linguistic communication (to speak in verses is, in fact, considered to be a symptom of psychiatric disorders - see "clanging"). Devices like alliterations, reduplications, rhymes, and assonances are used in languages only occasionally and selectively, and usually do not affect the meaning of an entire speech - unlike music, where the choice of meter drastically changes the expression of an entire music work (e.g., in the musical form of variations on a theme, one of variations often changes a meter, generating contrast between "march-like" courageous and "dance-like" pleasurable expressions in the rearrangement of the very same musical theme), so that a music-maker must commit to a certain meter in order to convey a desired content (e.g., it is simply impossible to create a march in the meter of 9/8).

e) Articulation engages different styles of connecting or disconnecting sounds. Each style can be thought of as a peculiar time-related idiom that obtains its semantic value through the implicit biological association with breathing patterns and relative lengths of a breathing group specific for a typical physical activity (the pattern of locomotion of which matches the length and articulation style of the articulation group). Thus, the ongoing staccato articulation is recognized by shortening the rhythmic values of musical tones by about $25 \%$ to make them distinctly separated, and it usually expresses fear, nervousness or cuteness/childishness (associated with the states of shivering, contraction, and helplessness). There are 9 simple 
articulations (staccatissimo, staccato, mezzo staccato, marcato, non legato, portato, tenuto, legato, and legatissimo) and there are many compound articulations that are generated by combining 2 or more articulations consecutively, thereby generating articulation groups (e.g., 2-note legato-staccato). The latter support music's capacity to suggest "expressive gestures", most evident in ballet music (e.g., 4-note staccato followed by 4-note legato in Tchaikovsky's The dance of little swans from Swan Lake expresses finesse and cuteness).

Such articulation "idioms" find no analogs in speech. Articulation of phonemes constitutes a remote homolog of musical articulations that contributes to expression in poetry through phonetic symbolism and onomatopoeia, as well as through alliterations and rhymes. However, such devices do not constitute an autonomous channel of conveying information different from lexic meaning of words in their prosaic use. Ideophones do contribute semantically to verbal phrases and sentences, but they only superficially relate to musical articulation. Even in singing, the latter bears no systemic connection to changes in configuration of one's mouth while articulating vowels and consonants. Instead, it relies solely on the temporal aspect of expression - on the extent of a gap that separates the onset of a tone to the end of a previous tone within the same group (e.g., short staccato vs. shorter staccatissimo), or, on the temporal extent of the emphasis placed on connection (e.g., barely connected tenuto vs. well-connected legato vs. overlapping legatissimo).

f) Dynamics engages a finite number of gradations in amplitude, each of which can be thought of as a dynamic idiom, semantically defined by the biologically ingrained association of intensity of sound with the intensity of an emotional state and/or proximity to the sound source (i.e., the extent of perceived emergency of an audio stimulus). Thus, fortissimo stands for the expression of extraordinary power, extreme danger that is very close, or an overwhelming emotion. Although speech also engages dynamics to emphasize the importance or urgency of certain ideas, verbal communication does not utilize discrete "dynamic classes" (such as musical pianissimo, piano, mezzo piano, mezzo forte, forte, fortissimo, crescendo, diminuendo, sforzando, fortepiano, subito piano, etc.).

Many languages regularly contrast stressed and unstressed syllables (in some cases, for differentiating between two homonymic words), thereby generating dynamic contrasts. But parallels with music do not extend any further beyond a basic capacity of placing dynamic accents here and there. And even such accentuation is not used continuously - ongoing dynamic contrasts obstruct the comprehension of speech (e.g., making one syllable very loud and another very soft, if applied consistently, would project the pathological impression). This is in contrast to music, where abrupt dynamic changes within the same music phrase only enhance the melodic and harmonic expressions (e.g., Beethoven - Symphony No.5, the 1st movement).

g) Texture engages a number of pitch/time components in consecutive and contemporaneous arrangement of sounds (i.e., texture simultaneously brings out "horizontal" linear relations between tones by segmenting patterns in parts and voices within a stream of sounds, while integrating the concurrently sounding tones into "vertical" components, such as chords). In essence, texture "chunks" the tones of a certain kind into vertical (harmonic) and horizontal (melodic) groups.

Such groups can be regarded as "textural idioms" suitable for a certain expression. Thus, melody, accompaniment, bass, or counter-melody in multi-part music constitute vertical "textural classes" (elements that can be engaged in building complex textures). Alberti bass is an example of the horizontal "idiom" that specializes in providing a smooth and pleasant accompaniment to the 
expressive melody (Alberti bass breaks arpeggio into the so-called "implied polyphony", where peak, trough, and middle points of every figure are marked as separate voices, thereby spreading the figuration over the wider registral range and making it appear lighter, while keeping it regular and, therefore, predictable and relaxed). Although semantically such "idioms" are not as clearly definable as melodic or rhythmic idioms, they nevertheless possess semantic specialization: e.g., a discrete bass part contributes to a sense of profoundness, whereas a figurative melodic accompaniment contributes fluidity, thereby making music appear less sturdy. Textural idioms often are used to convey contradictory and conflicting expressions (e.g., solid melody in the bass and fluid figuration in the treble register, as in the Etude C Major op.10 by Chopin).

This aspect of expression is unique to music.

h) Music form engages a number of musical themes in a sequential order (in monophonic, homophonic, and heterophonic textures) or in a contemporaneous counterpoint (in polyphonic or mixed homophonic-polyphonic textures). The number of themes in a music work can be regarded as a peculiar "idiom" that rhetorically determines the framework of expression for all the above-mentioned aspects of expression (e.g., it is impossible to convey a conflicting state in a work based on a single theme - two arguments are needed to generate the antithesis). Thus:

- a single-theme music work usually conveys a sustained emotional state that remains rather simple, homogenous, and unobscured - as in a typical prelude;

- a 2-theme work, as a rule, expresses a more volatile situation of the compensatory alternation between two contrasting states, one hesitant and another resolute - as in a typical song (lyrical and personal verse vs. affirmative and collective chorus);

- a 3-theme work acquires complexity and determination (the 3rd theme appears either as a compromise or a temporary distraction, so that the return of the previous themes appears as restitution); it is suitable for the presentation of dramatic interaction as in a typical march, where the opening theme is usually the most resolute, the second theme is less intense and/or busier, and the third theme deviates into a relaxing trio;

- a multi-thematic work with thematic development conveys the controversial or conflictual content as in a typical sonata, symphony, etc., where one idea collides with another idea, possibly obtaining supportive ideas, but only one idea prevails at the end.

The thematicity of music form resembles the rhetoric arrangement of speech into "paragraphs" to reflect the changes in topics or arguments. However, unlike the theme-based sections of music, the paragraphs are severely limited in their capacity to generate contrast to the preceding paragraphs (e.g., they cannot change a genre and/or style of presentation without breaking the continuity of a text) - not to mention that the polyphonic thematic arrangement of music (e.g., fugue) finds no equivalents in speech. Two speakers can accidentally speak on top of one another, but the manner of their overlapping is not regulated by any rules - unlike the rules of counterpoint that prescribe how pitches in different parts should be organized (and this counterpoint often engages 3-4 or even more, up to 40 parts, as in Spem in alium by Tallis).

The multi-channel nature of musical communication enables music to effectively convey conflicting, confused, and conflated ideas in a format that is easy to comprehend and memorize even for a layperson, without requiring much contemplation and deliberation (Fig.1). 


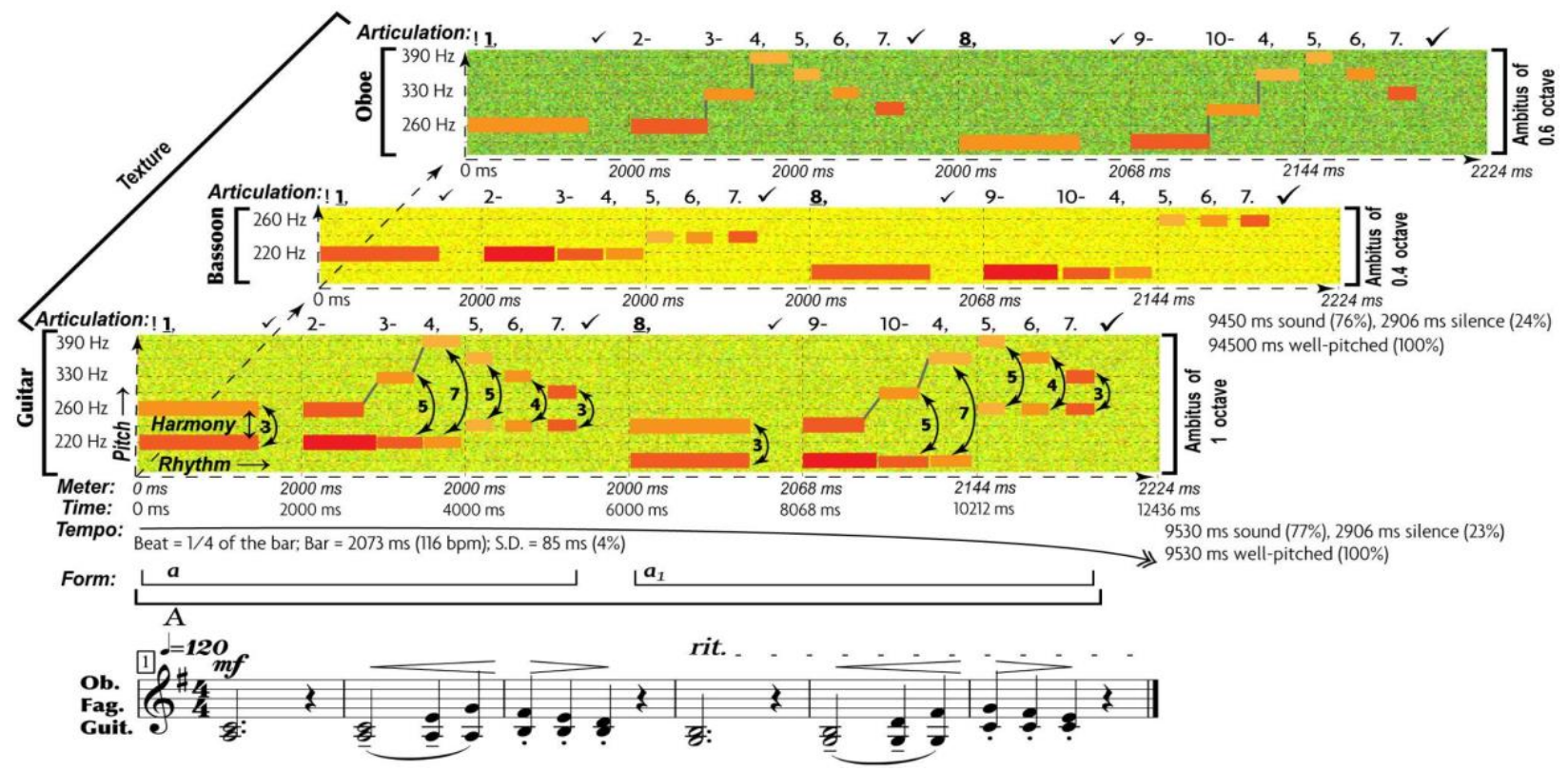

Figure-1. 11 aspects of expression of music. This visualization in the "musogram" format [18] elaborates the music notation at the figure's bottom. The lowest musogram (guitar) contains all the aspects marked out and named. Its horizontal axis (horizontal dashed arrow) represents time, vertical axis (vertical dashed arrow) frequency, depth axis (diagonal dashed arrow) texture. The latter joins all 3 musograms. Small colored rectangular bars indicate tones. Their relative vertical changes represent melody, with dash guidelines referencing exact frequency values. The changes in distance between the concurrent (superimposed) rectangles indicate harmony. The rectangular length represents rhythm. The breaks and ties between the consecutive rectangles comprise an aspect of articulation, indicated in a line above the frequency grid. Each tone is numbered, checkmarks indicate pauses (the longer the pause the larger the checkmark), and punctuation signs reflect the grouping of tones. The dash marks the connected tones (legato), comma-disconnected tones within the same phrase, period-the end of a phrase, and exclamation - the phrasal opening. Bold and underlined numbers mark anchor-tones (stressed by duration, dynamics, and frequency of occurrence). Thin gray lines represent connection: vertical between discrete pitches and tilted in the portamento. Color of the rectangles represents dynamics: from the loudest in yellow to the softest in blue. Thin vertical dashed lines indicate meterinferred from well-articulated occurrences of anchor-tones and longer rests. Tempo averages all metric units, in msec and beats-per-minute. The standard deviation shows how flexible the tempo is. A solid arrow with a double arrowhead reflects the tempo changes: ascending for accelerations, while descending for decelerations. The aspect of form reflects the thematic organization of the material, indicated by horizontal brackets and letters: thinner brackets and lowercase letters for motifs, and thicker brackets and uppercase letters for phrases. Each new material is marked by a new letter, and variationby a subscript number. Register is represented by the color of the grainy filling of the ambitus: from deep green for the darkest to light yellow for the lightest timbres. Timbre is indicated by the differentiation of the musical instruments and by greater thickness of harmonically rich tones. The complexity of this 11-aspect organization greatly exceeds the equivalent visualization of a speech stream in the form of "prosogram" [19]. The figure is reproduced with permission from [13].

Audio-1. Illustration of 11 aspects of music expression for Fig.1. http://chirb.it/IJsOgK

Each of the aspects can contain idioms that express different affective states, attitudes, attributes, and actions, thereby altogether presenting complex contradictory content - possibly, in the process of transformation (e.g., a smooth pleasant melody can be harmonized with dissonant chords and 
placed against a heavy aggressive bass line). This capacity is responsible for the reputation of music to be able to express what language struggles with. ${ }^{12}$

\section{Prevalence of integration over fragmentation}

The second principal difference is that music tends to systematically integrate multiple elements into a single perceptual entity, whereas speech tends to systematically fragment the stream of sounds into discrete and intelligible phonemes. This difference has to do with the inherent capacity of music to support the simultaneous production by multiple music-makers as opposed to speech that defaults to a single-speaker presentation format:

a. Compositeness. Even music that is strictly monophonic easily affords a monodic arrangement that contemporaneously joins multiple idioms. Thus, a single person routinely hums a melody with a self-accompaniment by tapping, finger-snapping, or clapping, which effectively generates two parts with autonomous idioms integrated into a single texture (e.g., multi-part rhythmic, metric, dynamic, articulatory, and textural idioms).

Speech does not engage anything similar to this - in fact, snapping fingers rhythmically to speech makes it sound like music (as in hip-hop). The non-lexical aspects of expression in speech, such as pace, rhythm, intonation, registration, usually only enhance the lexical meaning rather than convey additional information, and in rare exceptions, such as sarcasm, they do not form an autonomous channel of information, possessing its own patterns of expression. Humoristic and metaphoric meanings are detected not so much by prosodic means as by the context of previous speech and deictic reference to the circumstances of speech. Thus, rising intonation at the end of a sentence can be interpreted as a pitch pattern (similar to melodic contour of music) that signifies a question. However, the use of such a pattern does not come to contradict the lexical meaning of words and the syntactic construction of a sentence, which remain the primary means of conveying verbal information. In contrast, a monody in music, as a rule, integrates all idiomatic patterns present in multiple aspects of expression, which often differ in their semantics. Thus, rapping opposes the exciting power of diverse vocalized rhythms and the march-like directedness of binary instrumental metric pulsation, generating a peculiar impression of forceful struggle between rhythmic and metric expressions. Taking away either of them results in defeating the "rap" groove.

b. Voluminousness. Music possesses a parameter of voluminousness that is entirely missing in speech. Even a non-monodic strict monophonic melody can be performed by a varying number of performers. Thus, the melody of a verse can be sung solo, whereas the melody of a chorus by a choir, forming the contrasting timbral and textural arrangement of the responsorial type within the strictly monophonic texture. Similar contrasts can be generated between different vocal or instrumental settings (e.g., solo, a duo, a trio, tutti). Each of them is characterized by its own semantic contribution to other aspects of expression. For example, the contrast of solo and duo usually implies some kind of agreement between two individual affective states (two persons share something in common). The contrast between solo and tutti, on the other hand,

\footnotetext{
12 Here it would be useful to remind a number of famous quotations: "Where words fail music speaks" by Hans Christian Andersen; "Where words leave off, music begins" by Heinrich Heine; "Music comes to me more readily than words" by Beethoven; "Music expresses that which cannot be said and on which it is impossible to be silent" by Victor Hugo; "Music is the literature of the heart; it commences where speech ends" by Alphonse de Lamartine; and "Even if, in one or other of them, I had a particular word or words in mind, I would not tell anyone, because the same word means different things to different people. Only the songs say the same thing, arouse the same feeling, in everyone - a feeling that can't be expressed in words" by Felix Mendelssohn.
} 
implies that an individual sentiment somehow opposes the collective one. Hence, an acoustic parameter of voluminousness carries information related to sociality.

This parameter is simply inapplicable to speech. simultaneous pronunciation of the same text Even if multiple speakers memorize the same text and manage to pronounce it in sync, it would be very hard to follow what they say.

c. Lengthiness. The proneness of music for integration of sounds affects not only the vertical parameter (the aspects of harmony and texture) but the horizontal parameter (unleashing in time) as well. Thus, musical phrases can be enormously long even in a monophonic setting (e.g., J.S. Bach's Sonata for violin solo G minor BWV 1001, 1st movement, performed by Pikaizen, is 5 minutes long, yet it contains only 2 phrases). In polyphonic settings, such as in a fugue, phrases in individual parts overlap so that the entire music flow stops only at the very end of the entire music composition.

Verbal sentences, especially in oral speech, are severely limited in duration. A 2-minute-long sentence will appear as rambling, because it would impose a heavy burden on the verbal working memory. Not only the meanings of all words must be remembered to construct the meaning of a sentence (lexical meaning), but their manner of arrangement has to be considered (structural or linguistic meaning). As a rule, each sentence is supposed to express a single idea. Complex sentences are designed to specify circumstances, conditions, parallels, or causality of the principal idea. Changing an idea usually entails starting a new sentence. This is very different from musical phrases that are governed not by expression of a single concept but by expression of a single theme. Musical theme is often elaborated within a musical sentence, which constitutes a rule in many music cultures and styles (e.g., Baroque music). In non-Western cultures, musical phrases also can be excruciatingly long (e.g., in maqam or raga). Musical phrase is more of a process, whereas verbal sentence is more of a statement.

The integrative power of music makes it possible for music cultures to support much greater complexity in sequential (horizontal) and contemporaneous (vertical) arrangement of sounds in comparison to speech. The latter is severely constrained by the necessity to emphasize the phonemic contrasts, while keeping them well-articulated in the output by a single speaker, therefore adopting a strict principle of taking turns. Talking on top of someone else is considered an obstacle to normal verbal communication in most cultures, while for music, singing and playing an instrument on top of another performer constitutes a universal norm.

The other constraint of speech has to do, as noted, with the limitations of short-term memory that is required to keep storing lexical meanings for each subsequent word in a sentence before the meaning of the entire sentence can be inferred. Short span of short-term memory necessitates the strategy of caching the information within the sentence and emptying the cache at the end of that sentence, retaining only the memory of the point of a sentence. Comprehension of speech consists of cycles of such caching. However, this strategy of chunking and compression remains effective only up to a certain limit - at some point a listener inevitably forgets the points of the first sentences in a speech and loses track of the entire speech. Music is free of such constraints, because its main purpose is to enable listeners to experience musical emotions rather than to convey concepts. For this reason, listeners have no problems comprehending a 2-hour long symphony of Western classical music (Mahler - Symphony No.3) or even a day-long nubah of Arabo-Andalusian music tradition [20]. 


\section{Relatively low information density of a music work}

Since music specializes in the transmission of emotional information, while speech - referential information (although expressive uses of language are also common), they both drastically differ in relation to the informational density (number of meaningful patterns in the stream of information per unit of time):

a. Contemplativeness. Continuous verbal interaction is usually characterized by high informational load, where speakers exchange information in an ongoing manner with unpredictable outcomes, so that the redundancy or the slow pace of transmission are viewed as faults. A typical conversation shifts between dozens of subjects that address various aspects of the same topic and can switch from topic to topic a number of times. Continuous music, in contrast, relies on a long exposure of the same musical idea in order to secure entrainment and emotional contagion. Even the most "restless" composers, such as Schumann or Mendelssohn, have many works where a single affective state is exposed for 3-4 minutes. Unlike speech, music is contemplative by design. Non-Western music traditions tend to be even more contemplative than Western classical music of the Common Practice Period - e.g., an entire raga or a maqam is typically dedicated to the exposure of a single musical emotion, which usually takes at least 10-20 minutes.

b. Redundancy. Contemplative design of music puts in place many devices of arrangement that would be totally foreign and even detrimental to speech communication, had they been somehow applied to speech. Thus, the ostinato accompaniment (a sustained repetition of the same pattern in a supporting part) and multiple exact repetitions of the same tune are exceedingly common for modern folk and popular songs as well as many forms of traditional music. The principal idea behind the use of such musical devices is to prolong the exposure of a thematic material while adhering to the ongoing reproduction of the same structural pattern. Both functions are designed to make music more predictable and therefore likable, and to secure effective emotional contagion and entrainment for the audience. As a result, it is not uncommon to have a 3-minute-long song made of numerous repetitions of just 10 tones (see the example of Audio-11 in 7.5).

Such high rates of repetitiveness are atypical for a speech delivered in any known language. A rare exception might be the format of magic spells and incantations that usually border with music. On a lexical level, tautology generally is considered a fault of style. A very limited use of repetition for specific effects is regulated by rhetoric figures: a single repetition in antimetabole, antanaclasis, anadiplosis, epanalepsis and polyptoton; 1-4 repetitions in epizeuxis, 3-4 in epistrophe and mesodiplosis, 2-8 in anaphora and diacope [21]. ${ }^{13}$ Compared to music, this level of redundancy is insignificant. Thus, a popular song "Superstition" by Stevie Wonder is based on about a hundred repetitions of the same riff [22]. Even in the tradition of classical music, where frequent mechanical repetition of the same brief thematic material is generally regarded as bad taste, there are genres based on exact reproduction of the same theme in the same part - e.g., passacaglia (for example, 21 continuous repetitions in the famous Passacaglia and Fugue in C minor BWV 582 by Bach).

\footnotetext{
${ }^{13}$ More frequent repetitions of the same pattern of verbal expression tend to make speech acquire musical traits, as evident in such genres as street cries, forest calls, magic incantations, shamanic healing rituals, moherese, herders' conversations with domestic animals, or animal spells for sick domestic animals. In all these examples, a repeated pattern that expresses a particular affective state or an attitude becomes prolonged, directing the listener's attention to the tonal properties of sound rather than the lexical meaning of words - which makes the sound music-like.
} 
Formulaic solo songs of indigenous Siberian ethnicities can be repeated as many times as the accompanying activity lasts, which for riding songs can take hours, if not days [23].

c. Multifunctionality. Prolonged exposure of the same thematic material in multi-channel transmission equips music with the unparalleled capacity to reserve each channel for specific functionality. Potentially, each of the aspects can convey different things through proprietary idiomatic patterns. In fact, this arrangement has become a standard for the popular music industry, where most forms of music are set to the ensemble of 3-6 performers, each specializing in a particular aspect of expression: solo vocal on melody; group vocals, keyboard(s) and rhythm guitar on harmony; rhythm guitar and percussions on rhythm; solo guitar on alternative melody and melodic figurations, extra keyboard on pedal-like sustained pads, wind instruments on accentuated "licks", drum set and bass guitar on meter and ostinato riffs - coloring each of the parts with idiosyncratic timbre, dynamics and articulation [24]. This specialization in musical expression has established professional specialization and the consensus for which set of skills and which repertory of patterns a performer on a particular instrument, or a singer, has to know in order to find a job.

Nothing similar to this exists in verbal communication. Although it shares with music the expressive aspects of rhythm, pitch, tempo, dynamics, registration, and articulation, its primary focus falls on the domain of timbre - namely, on phonological contrasts between phonemes, syllables and words' boundaries, which requires strict solo delivery and entirely prohibits the creative aspect of composition of parts [25].

The apparent contradiction between the high information load of multichannel musical delivery and the reduction in information density due to contemplativeness and redundancy of music is explained by the specialization of music on emotional communication. The need to secure effective emotional contagion and entrainment pushes music-makers to ensure that the exposure of a specific musical emotion is never too brief, sketchy, or transitional. The use of new material within each aspect of expression must be spared - preferably, 1-2 characteristic idioms. As a result, music loses to speech in the informational density of any single aspect of expression but wins in the combined informational density of all its aspects at any specific moment of time.

The densest musical aspect, melody, is still inferior to speech in its capacity to reflect multiple changes in "real time" without affecting ineligibility of musical communication. Thus, music comes no way close to how sport TV commentators manage not to lag behind even in very animated events, such as football matches. This is because music demands more time to adequately present musical emotions and to make sure that listeners have an opportunity to experience them. However, the joined load of all aspects of musical expression is capable of capturing all sorts of shades and hues in the affective states, even if they are most perplexed, mixed, convoluted, and conflicting. Music can show their transformation in time between multiple actors. This extent of theatricality is simply beyond the reach of verbal expression. ${ }^{14}$

\footnotetext{
${ }^{14}$ Here it would be appropriate to point out the difference between a theatrical play and the opera on the same plot, e.g., Le roi s'amuse by Victor Hugo vs. Rigoletto by Giuseppe Verdi. The verbal format of restricting the information flow to a single speaker at a time severely undercuts the capacity of Hugo to reflect nuances in the interaction of his protagonists on stage, while keeping a fast pace of dramatic action. The multichannel format of music, on the other hand, enables such a spectacular display of 4 contemporaneous contrasting affective states as in the famous Quartet of the III Act - at the cost of noticeable slowing of the dramatic development.
} 


\section{Formative power of musical theme}

Musical theme is yet another attribute of structural organization that distinguishes music from speech. Musical theme can be defined as a segment of music that makes sense on its own, does not sound interrupted, expresses a specific image, character, or a musical emotion, and enables the recognition of a specific music work upon its audition [26]. In musical literature, "musical theme" is often equated with the subject of a sentence, which is commonly the topic (or referential scope) of that sentence. However, this analogy is rather loose: there are many music works based on a single theme, whereas each sentence usually has its own topic. More appropriate is the analogy between a musical theme and a discourse topic (i.e., what a text fragment or conversation is about): both secure the coherence of an entire composition and the relevance of its content, supporting its subsequent identification. However, there are still a few important distinctions between the two.

Musical themes differ from discourse topics by the capacity of any of the numerous aspects of musical expression to provide a salient pattern that makes this music identifiable. Although melody serves this purpose most commonly, a theme can also be recognized by a harmonic progression (e.g., any chaconne of classical music or any jazz theme), rhythmic figure (most of social dances), metric formula (some genres/styles, such as bossa nova that features the grouping $8 / 8=3 / 8+3 / 8+2 / 8$ ), articulation formula (most etudes, e.g., staccato, legato, and mixed combinations), and textural formula (many preludes, e.g., melody in the bass and the chords above it). In contrast, sentence or discourse topics do not exhibit such a rich multidimensional nature. Although sentence topics can be marked by intonational changes, particles, affixes, or specific syntactic constructions, languages usually exploit one of these resources only. For this reason, the presence of thematic organization [27]: repetition, variation, contrasting change, and recapitulation (i.e., the return after something else) of specific thematic material in all or a few of the above-listed 6 aspects of expression throughout a complete audible "text" - should be taken as an indication that the auditory artifact under question is music rather than speech.

\section{Absolute pitch}

Pitch-oriented music may not have been the first form of music to emerge, but it has been steadily acquiring a dominant status over the timbre-oriented music all over the world, with ever increasing pace, especially lately, transforming timbre-oriented traditions into pitch-oriented [28]. The result of this global tendency is the growing spread of the capacity to perceive absolute pitch, of which no record exists prior to the 18th century, and the earliest records all point to the emergence of Western tonality as the underlying foundation in the genesis of the concept of "perfect ear" [29]. The latter could constitute a late adaptation to the grown importance of frequency discrimination in industrial societies, or a new cultural tradition closely related to the rise of "prodigism," or, both. The estimates of the commonality of "natural" (aka, "untrained") absolute pitch hearing vary from $0.0001 \%$ of general population to $15 \%$, whereas the "trained" perfect ear (less reliable and more limited across different timbres than the "untrained" perfect ear) can be developed by virtually anyone who regularly follows the adequate methodology of ear-training (ibid.). At any rate, the possession of absolute pitch hearing affects the perception of music structures in 2 ways:

a. Concert pitch reference. All pieces of music created by those who possess absolute pitch hearing share the standardized tuning of pitch classes (e.g., referenced to A4=440 Hz) across different pitch-class sets. In effect, all "Do's", "Re's", "Mi's", etc., obtain their absolute pitch values no matter to which key and/or mode they belong. For possessors of absolute pitch, these absolute values become more important means of identifying the tonal organization in music 
than relative framework of reference, such as the recognition of intervallic distances between different pitches and of degrees in a key or a mode (since absolute pitch processing requires no attention and occurs automatically).

There is no place for such a method of frequency analysis in perception of speech. Relative stability of pitch levels for vowels, known as "intrinsic pitch of vowels", is sustained only on average (with high fluctuations in standard deviation) per individual, substantially varying between different speakers [30]. Neither intrinsic vowel pitch nor lexical tones of tone languages provide an equivalent of absolute pitch in music, because they do not support identification of meaningful particles of communication by means of frequency analysis. The critical overview of geographic and historic distribution of absolute pitch across the globe suggests that absolute pitch is an exclusive musical phenomenon that has no linguistic origin [29].

b. Qualia of specific keys. Paradoxically, the standardization of pitch values across pitch sets, which uniforms all keys within the equal temperament tuning, by the same token enables the absolutization of the pitch values within each specific key outside of equal temperament. Division of an octave in 12 equal semitones, in effect, concisely equalizes all keys, whereas Pythagorean and meantone tuning systems make some semitones slightly larger or smaller than others, prioritizing certain interval classes - which makes the sound of one key differ from another key [31]. Pythagorean tuning, prevalent in civilizations of antiquity [32], but abandoned by Western classical tradition once it committed to the polyphonic theories of composition [31], was revived in the 18th century after the adoption of temperament. This is to compensate for the loss of distinctions between the "equalized" interval-classes within the same key, which led to the decrease in melodic expression, especially for strings and vocals [33]. Pythagorean tuning emphasized the differences between major and minor, as well as between augmented, diminished and natural intervals. Therefore, Pythagorean intonation has become a status quo for the melodic aspect, whereas "just" (aka pure) intonation has set the model for the harmonic aspect of expression [34]. The use of temperament has been restricted to keyboard instruments (piano, organ) for its shortcomings in expressive intonation [35].

Within this framework of tuning, all forms of solo and ensemble music that do not include piano or organ make it possible for possessors of absolute pitch to recognize a unique sound of each key due to the placement of slightly sharpened or flattened intervals on a specific degree of a key. The functional relation of such an interval to the tonic of a key determines the qualia of a given key. The semantic definitions of each of the keys were codified in the influential treatise by Christian Schubart Ideen zu einer Aesthetik der Tonkunst (1806). For example, C minor was considered suitable for declaration of love and Romantic lamentations, whereas $\mathrm{C}$ major for expression of innocence, naivety and simplicity; B minor for submission to one's fate and patient awaiting, whereas B major for intense display of wild passions [36].

These semantic distinctions find no analog in speech.

Overall, it seems that the stability of absolute pitch values across different auditory artifacts can serve as a reliable indicator that the latter constitute music and not speech. The same applies to the situation when different auditory artifacts adopt the same reference pitch-class while demonstrating a close similarity in semantic qualia (i.e., numerous modern sound-recordings of solo vocals that reference $31 \mathrm{~Hz}$ and its multiples, aka "B", as the most stable tone and sound exceedingly tragic are likely to constitute music). 


\section{Semantic capacities of intervallic typology}

Intervallic typology provides a unique source of expression in music and controls the structural uniformity within a chosen manner of distinction between the interval-classes [37]. Thus, anhemitonic pentatonic music (i.e., an intervallic system that does not use semitones at all) is common for expressing stable harmonious, balanced, overall happy (even minor keys sound contended), and contemplative characters with very limited means of generating tonal tension, contrast, and diversity. Diatonic heptatonic music (an intervallic system that entails only 2 semitones, definable through the series of 6 intervals of perfect 5th) is common for expressing contrasting states of positive and negative emotions that remain distinct, overall optimistic (even minor keys sound pleasant), often simple, childish, or naive. Chromatic music (multiple consecutive semitones) is common for expressing intensely negative emotions, unstable or conflicting emotional states, or some struggle. Microtonal music (intervals smaller than a semitone) is common for expressing extreme suffering, grief, tension or, on polar opposite, refined pleasure and elation.

The commitment of a music-maker to any of such types of interval-classes pre-compositionally determines the semantic scope of a music work. Moreover, switching from one intervallic type to another within the same work (e.g., from a Western tonal key to the whole-tone musical mode or to an anhemitonic pentatonic mode, as found in many compositions by Debussy) presents a unique expressive means akin to modulation from one musical mode to another.

Such means of expression falls completely out of scope of verbal communication. The presence of intervallic uniformity and contrasts between a few intervallic types can serve as an additional criterion of distinguishing music from non-music in difficult cases.

\section{Formative power of the concept of euphony}

Euphony serves as a universal fundamental formative principle of tonal organization in music that bears little to no semantic contribution to the task of conveying emotional information. Musicusers always combine certain types of sounds that give a "good" match. No matter which expression is communicated through music, musical modes and keys are generated based on the sensibility of music-users to the concordance of the preferred "tone-classes" (pitch-classes for frequency-oriented, timbre-classes for timbre-oriented, time-classes for rhythm-oriented forms of music) and their suitability for a desired expression. Thus, the notion of "goodness" forms music.

The pleasure from listening to music, as a rule, underlies the expression of non-pleasurable affective states, such as grief, melancholy, fear, anxiety - and this is what distinguishes musical emotions from real-life emotions. Listeners routinely describe their experience of listening to sad music as pleasurable. This is as though musical representation of negative feelings somehow idealizes and elevates them, so that their expression can be aesthetically appreciated. The concern for euphonic uniformity in the chosen style of arrangement of sounds is central to tonal organization of music, applicable to all aspects of expression, but most obvious in those aspects that deal with the frequency domain (perhaps, since melody is the most expressive aspect of many, if not most music cultures).

The notion of euphony is embedded in the concept of "musical mode." Although the notion of concordance of tones in a musical mode received only a few mentions in the Grove Dictionary's entry on "mode", the issue of tonal euphony has constituted the prime object of discussion by great many music theorists from Antiquity to the 19th century [38]. Concerns for concordance of the 
combinations of pitches also underlie the conventions of tuning musical instruments in numerous folk non-Western traditions [39]. In Russian musicology, the very term "musical mode" (Russian, "muzykal'nyi lad") implies concordance ("lad") that is pleasant to senses. The formal definition of musical mode has been coined by Yurii Kholopov in the USSR Encyclopedia of Music: "mode is the concordance of tones, pleasant to ear, and manifested in systematic relations of pitch classes that are joined in a set by means of subordination to a specific pitch class (or a few pitch classes) and by combination rules that regulate construction of melody" [40]. Following the legacy of Boleslav Yavorsky (1908), the concept of musical mode is a part of the theory of musical intonation [41]. Developed by Asafiyev, this theory holds a musical mode as a "container" of intonations most important for a given musical culture [6] - a convenient model for understanding the tonal organization of traditions based on oral transmission [42].

The concept of musical mode is paramount for studying musical syntax: why certain combinations of tones make sense, while others do not, and how music-makers actually construct their music especially in music systems other than Western tonality. The existing corpus of research literature on the psychoacoustic mechanisms responsible for the integrity of a music work (such scholars as Krumhansl, Huron, Lerdahl and Jackendoff) covers mostly the hierarchical structures of Western tonality. The other forms of tonal organization have been gathered by musicologists under the umbrella of "modality" ever since Alexandre Choron introduced it as the antithesis of tonality, in 1810, characterizing both as contrasting schemes of musical thinking [43]. In his famous Esquisse de l'histoire de l'harmonie, François-Joseph Fétis placed Choron's antithesis into the evolutionary framework - as different stages (tonality succeeding modality) and mental cultures of integrating musical tones according to the sensibility of music-users. This evolutionary perspective tied schemes of tonal organization of music with different psycho-cultural models of euphony. Fétis' authority as the author of the first European music dictionary has established the tradition in Western musicology to draw the history of harmony as periods of euphonization of different interval-classes (e.g., appreciation of the interval of a 4th by Ancient Greeks and Romans, followed by its dethroning and replacement by the interval of a 3rd in the 15th century Western Europe). This history has been presented as the history of the antithesis between consonance and dissonance [44].

The phonology of languages shares many common features with music - timbre-oriented instrumental music (indigenous Jaw Harp traditions of East Asia) is often based on vocal systems very similar to languages, including combinatorial syntax akin morphology. However, matching of morphemes in languages applies only to a very limited number of their combinations. Pitchoriented musical modes, in contrast, are distinguished by the concordance of all combinations of pitch-classes along a selected acoustic attribute. This holds true even in those music cultures that adopt acoustic roughness as a model of euphony - as in the so-called Schwebungsdiaphonie [45]. For this reason, people routinely enjoy listening to hour-long music recitals of music from a distant foreign culture, whose conventions they do not understand at all, whereas the enjoyment of listening for an hour of unfamiliar foreign language (even if this is the master poetry) is not nearly as common.

Whether consonance and dissonance are seen as a biologically ingrained phenomenon, a cultural tradition, or both, the truth of the matter is that their existence is fundamental to music and based on the propensity to appreciate the euphony of sounds [46]. This nearly universal association of music with enjoyment from hearing certain combinations of sounds finds support in neuroscience - music constitutes a unique medium of appreciating the beauty of sound per se [47]. Inability to 
enjoy music is exceedingly rare amongst humans, indicating that music evolved as direct neural pathwork, designed to interconnect auditory sensory organs with the emotional reward circuits in the brain [48]. Aesthetic appreciation of tonal organization of melody, harmony, and other aspects of expression characterizes music and is uncharacteristic for speech communication, apart from certain poetic styles (e.g., gongorism) and, perhaps, some rare phenomena like "servant" or "decorative" morphology in such languages as Khmer [49]. It is this imperative of appreciation that explains contemplativeness and redundancy of music: people love to listen to the pleasurable combinations of tones again and again - in contrast to their perception of speech, where they look for novel information and become annoyed by multiple repetitions.

All in all, the above-listed 7 traits together provide a reliable way of distinguishing music from speech solely on the grounds of structural analysis of the sounds - disregarding the information about behaviors and goals of actors engaged in auditory communication.

\subsection{The functional typology of music}

After providing a definition of music and identifying specific features of music, we shall list the types of functions typically performed by music, according to the ethnomusicological, cognitive, and sociological data [12,13,17,29,50-67]. Active (performance) and passive (listening) uses ${ }^{15}$ of music can be grouped in 14 general operational types, following the criteria of cross-cultural stability of their pragmatics and presence of specific structural means to distinguish each type of use. Below is the list of them, from the most to the least common, as currently observed.

1) Hedonistic stimulation. The pleasure from music supports one's well-being:

a) enjoying music per se - currently, the most popular form of passive listening and active musicmaking as a hobby, personal as well as collective,

b) enhancing some extra-musical activity - exceedingly popular in passive background listening at recreational events and while doing chores, such as shopping, as well as in personal active use (e.g., humming or whistling a tune while walking),

c) compensating for suffering some form of cognitive dissonance or stress - most common in form of listening to favorite sound records while executing tedious tasks;

2) Emotional communication. Music reflects one's current emotional or/and physical state:

a) directly signaling to listeners in live settings - the prevalent use in folk cultures,

b) projecting an ideal affective state suitable for a specific social occasion (e.g., birthday, national or religious holiday) - this is common in passive and active uses, especially family,

c) self-reflecting and exploring one's own personality - typical for passive personal use,

d) expressing on behalf of a fictional protagonist of a music work (e.g., a character who is as though speaking to the audience through the lyrics of a song or is represented by the musical movement of an instrumental piece of music) - active use only, a performer impersonates a

\footnotetext{
15 We do not distinguish between the terms "function" and "use" the way Alan Merriam did [68], and how it became customary in Western ethnomusicology. We consider "use" a mere application of a specific "function" rather than adopt Merriam's antithesis of use and function as, respectively, "situation" versus "reason" for engaging into a musical behavior. We are interested solely in identifying the principal ways humans interact with certain types of music structures across different cultures.
} 
character akin to an actor playing a role; this is the default of Western classical, theatrical, film, and popular forms of music, as well as for children make-belief games;

3) Emotional regulation. Music controls and regulates one's affective state, mood, disposition, or attitude in order to attain certain goals:

a) triggering a mood/disposition that would be beneficial for one's activity - by far the most common passive application (e.g., listening to heavy metal during heavy workouts in a gym),

b) reinforcing an already present affective state that agrees with a person's goals (e.g., listening to relaxing music when feeling tired in order to rest) - typical for passive use of music,

c) venting out an intense emotional experience by listening to the music that expresses it or by performing such music (e.g., playing heavy metal guitar when angry),

d) getting rid of an undesired mood/disposition by listening to a music or performing music that expresses the opposite affective state (e.g., listening to happy music when feeling sad),

e) diversifying one's daily emotional experience in case the latter tends to be deficient, monotonous, or too repetitive - common for patrons of musical theater and performers,

g) modifying one's emotional state to bring it in line with a social group in a collective activity or event - very common for patriotic and religious music,

f) virtual companionship - in passive use, often a favorite performer or composer is viewed as a virtual companion in sharing the same affective state, in active use - a composer;

4) Interpersonal bonding. Music secures close inter-individual bonding and mutual care:

a) mediating between a caretaker and an infant - the most common cross-cultural forms of motherese, lulling and musical playtime,

b) mediating between a person and a supernatural power in sacrificial and tributary religious rites, designed to secure a favorable outcome for a person (e.g., a prayer) or a congregation (e.g., a hymn) - such rituals have been extremely important throughout cultural evolution,

c) supporting romantic relations in small-size group musicking (e.g., romantic Jaw Harp duets) and social dances - especially the latter have been nearly omnipresent throughout history;

5) Coalition status display. Music displays the coalition membership of an individual:

a) manifesting one's social identity through the choice of music genre/style - active and passive use of music in old times (personal song) and at present (peer-group membership);

b) projecting a wish-to-be identity - a byproduct of the above, designed to enhance one's public image by displaying the patronizing attitude to socially prestigious forms of music,

c) affirming one's cultural and/or ethnic identity as means to empower oneself mentally and overcome difficulties and challenges in life - omnipresent use, active and passive,

d) signaling the coalition quality to other members of the same social group, distinguishing members in status - this function used to be important in collective music-making in preindustrial societies, rewarding more skillful members; the remnant of it can still be observed in idolization of "stars" of popular music;

6) Compliance to norms. Music disciplines the long-term attitudes, preferences, and goals of a person in accordance with some ideals: 
a) making an individual feel unified with a deity, supernatural force, nature, and a congregation of worshippers in order to gain power, confidence, harmony, and stability - this use is close to emotional regulation but bears spiritual rather than social orientation (common use of metric idioms to evoke the state of trance or meditation),

b) bringing an entire social group to experiencing the same affective state suitable for collective execution of some important task (e.g., warfare) - this use differs from emotional regulation by having collective rather than personal orientation,

c) homogenizing and ritualizing behavior within a social group by stimulating compliance with social norms - this use was important in pre-industrial societies, especially of the East;

7) Recreation. Music provides diversion, entertainment, and fun:

a) sporting recreational activities, such as tongue-twister songs, ditties for social games (e.g., dice), onomatopoeic imitations, phonation play (e.g., katajjaq), musical jokes/humor (e.g., assalalaa), music contests - active use, common for pre-industrial cultures,

b) adult's playing with children, such as nursery rhymes and ditties - common in all societies,

c) children's playing, such as musical babbling of infants, solitary vocal improvisations by preschool children, and social play-songs (counters, jumping songs, etc.) - universal use,

d) exploring a peculiar musical instrument (e.g., kazoo), usually solo - less common use,

e) appreciating virtuosic, timbrally unusual, or programme music - passive, common use;

8) Self-promotion. Music puts one's faculties at public display to one's advantage:

a) displaying features that are valued as cultural virtues in individual, family, or larger social group's musicking (e.g., male strength, female grace, children cuteness) - this active use was more important in pre-industrial societies,

b) the same as above but for self-affirmation and confidence - active solitary use,

c) capitalizing on one's proficiency in performing technique (vocal and/or instrumental) - this function became pronounced in Ancient Greek culture and ever since it is still highly regarded in modern industrial societies;

9) Physical aid. Music supports a characteristic pattern of physical motion by means of rhythmometric, articulation and melodic patterns of music performed along with such motion:

a) facilitating the execution of demanding physical work (e.g., harvesting, planting, rowing), achieved via musical genres (e.g., agricultural work-song, chanty, or march) - active use was common in pre-industrial societies, presently exists also in passive form (parade marches),

b) matching a pattern of playing a game by a group of children - common active use,

c) accompanying the actions of make-belief characters in solitary playing dolls or soldiers - id.,

d) supporting the execution of a sport exercise (e.g., gymnastics, figure-skating) - id.;

10) Learning aid. Music helps memorization of knowledge important for success:

a) singing songs created by adults for children as mnemonic devices for remembering important information (e.g., alphabet songs) - this active use seems to be rather recent,

b) singing work-songs that contain instructions for executing a certain work (e.g., weavers' songs of Central Asia and China that name operations necessary for weaving a rug) - rare, 
c) singing traveling songs that specify landmarks or encode a scheme of orientation optimal for a given topographic environment - rare (indigenous people in Siberia and Australia);

11) Contemplating an event. Music evokes the imagery of an important occasion, place of interest, or monument - this use can be both active and passive, more pronounced in seasonal and special occasion genres of music (e.g., birthday, graduation, wedding, Christmas music), when the latter are performed off-season; in pre-industrial societies, there are often vocal and instrumental compositions specific to festivals (opening or closing), sporting events (wrestling music), and venerating important landmarks (sacred hymns and incantations);

12) Calling to action. Music conveys specific commands, especially at long distances:

a) codified use of music motifs to trigger specific responses from people (e.g., military bugle signals, or patterns of bell ringing in the Orthodox Church),

b) specific herding calls, whistles, and patterns played on musical instruments to trigger specific responses from domestic animals (cows, horses, reindeers, goats, sheep),

c) specific vocalizations believed to evoke a desired action of supernatural forces (spells, incantations, shamanic kamlaniye);

13) Conservation of memories. Music preserves one's integrity and one's relation with close people by means of common reminiscences of the valuable past: ${ }^{16}$

a) every individual acquires a repertory of music pieces associated with the memory of the beloved and of the self in a distant past, so that the repetitive listening to them or performing them for oneself or others preserves one's integrity,

b) the same as above but collective (e.g., former classmates),

c) singing during the episodes of prolonged solitude and isolation in order to maintain selfawareness and integrity of thinking or to stay awake [12],

d) high mnemonic capacity of music is reflected in earworms (an exclusive musical phenomenon), widely used in music therapy to treat mental disorders that affect memory;

14) Personal profiting. Today music is often used to earn money or fame. This function, not very typical for unprofessional folk traditions, ${ }^{17}$ has been evolving since the late Middle Ages within the Western classical music and developed its own repertory of music idioms called to make listeners like this music and consume it again. Such idioms allow listeners to identify socalled "commercial" forms of music by ear (numerous postmodernist styles of composition can be regarded as implementation of this function).

\footnotetext{
${ }^{16}$ Despite this use being one of the most widespread reasons for modern-day listening to certain compositions as well as playing them, we listed it at the end. This is because of its individual and subjective nature. This use seems to lack dedicated structural features that could distinguish it from other uses. Promising is the research of the earworm phenomenon that is characterized by the presence of certain features in patterns of pitch and rhythm.

${ }^{17}$ Although the idea that music can be owned and traded for some goods or currency is quite wide-spread in indigenous traditional cultures over the globe, music-making "for profit" is usually not regarded as a personal commercial occupation in societies that do not have professional musicians [68]. Traded music usually belongs to a community, a clan, or a family. Personal songs also could be traded but they were not manufactured with the purpose to sell [69].
} 


\subsection{The dependencies between music functions}

Importantly, 14 operational functions of music listed above reveal clear dependencies. Thus, function X (calling to action) relies on functions IXa and VIIIa: to recognize a musical signal and distinguish its meaning from another signal (e.g., bugle "retreat call" vs. "assembly call") one must remember that signal (conservation of memories) and retrieve it at will (contemplating an event). Rearranging the order of the operational functions so that every dependent function goes after its determinants will reveal the plausible evolutionary succession functions that can be plotted against the structural features which distinguish music, identified in Section 2.3. This, in turn, will suggest the lineage in the evolution of tonal organization of music (Fig.2).

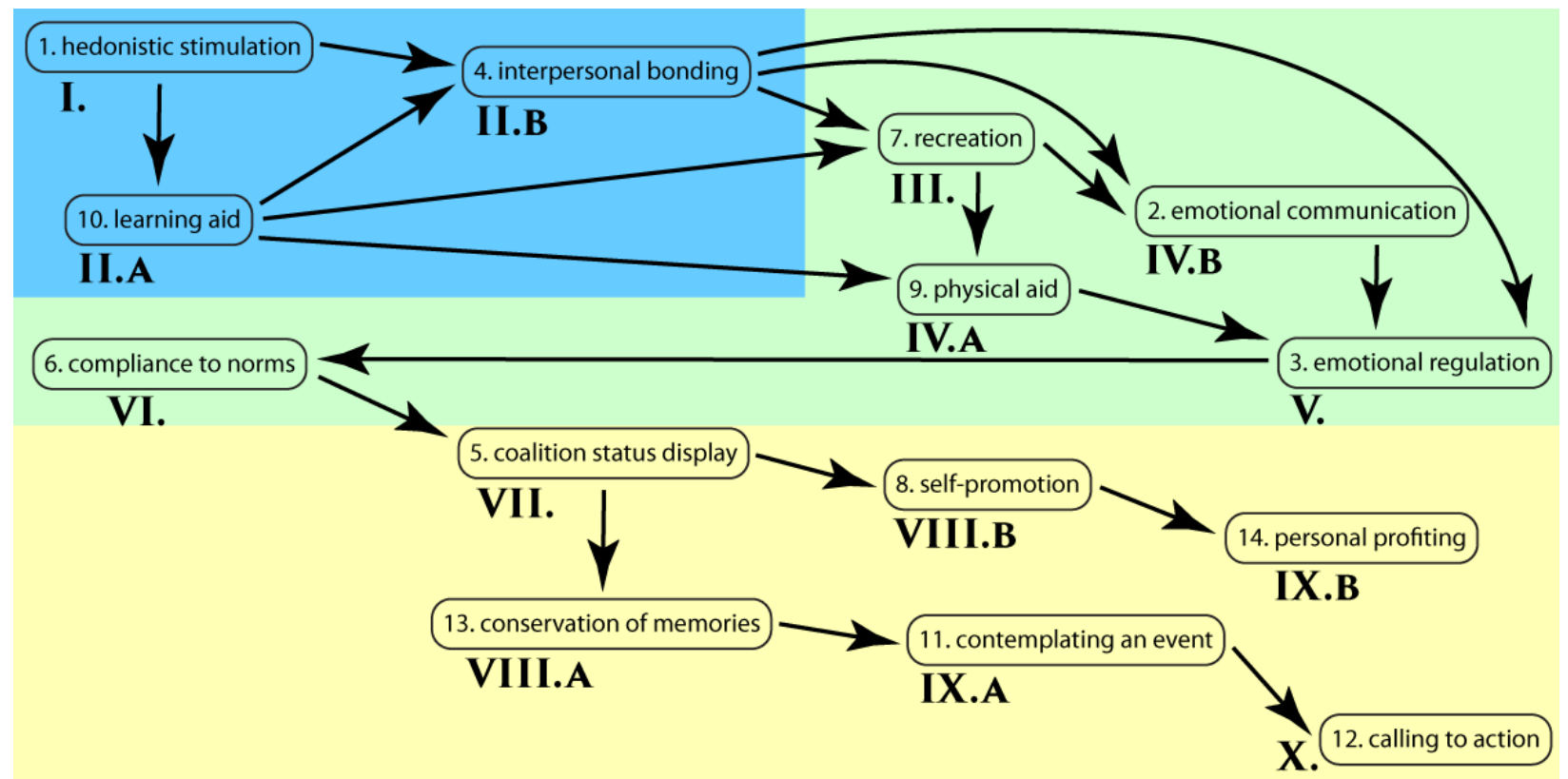

Figure-2. Evolutionary development of operational functions of music. The boxed names of each function are numbered according to their current popularity. The roman numerals below each box indicate the hypothetical order of all developmental phases in the evolution of music. Arrows reflect the derivative relations between the functions. A blue rectangle at the left top encloses 3 functions that are undifferentiated from verbal communication and characterize the "musilanguage" [2]. A green rectangle marks 5 functions that characterize the acquisition of music skills in early childhood in their typical succession. A yellow rectangle at the bottom encloses 6 functions that characterize adult and adolescent common uses of music. This figure is original.

The most likely candidate for the primordial function is hedonistic stimulation - this function underlies all other functions and can be considered fundamental to the experience of music [63]. Moreover, numerous animal studies have reported reduced stress behavior (including reduction of aggression), increased positive behavior, and beneficial physiological responses to human music [70-77]. Such effects of music can be attributed to the oxytocin and vasopressin neurochemistry of the reward system shared by humans and animals [78]. The rewarding response to processing music might be reinforced chemically through the mesolimbic dopamine pathways and by electrical stimulation of subcortical regions, involved in emotion and reward processing common for many animals, and of cortical regions that are implicated in complex cognitive function [79]. Basic neural mechanisms in the brainstem and auditory cortex seem to be functional at birth, allowing human infants to distinguish musical sounds from other auditory stimuli [80]. By the age of 6 months, infants respond to speech of caretakers by attempting to socialize, yet quietly 
contemplate musical sounds [81]. At about this age, they display liking for some of the acoustic attributes of musical sounds [82].

All of these suggests that the hedonistic function constitutes a starting point in musical development and probably is biologically ingrained. This hedonism is of major concern for us, since the emotion of pleasure constitutes an antipode to anger (i.e., an acute experience of displeasure). Experience of happiness is known to promote liking, whereas anger - to decrease liking, thereby directly affecting the willingness of individuals to interact [83]. The anti-aggressive influence of hedonistic experiences is evident in behavior of domestic animals as opposed to their wild conspecifics. Changes in the dopamine system have been found in domesticated animals $[84,85]$. Similar physiological mechanisms seem to have taken place in the evolution of humans. There is evidence of selection of pathways related to dopaminergic synapse [86]. Likewise, domesticated animals exhibit higher densities of oxytocin cells [87]. Oxytocin inhibits stresstriggered activity of the hypothalamic-pituitary-adrenal (HPA) axis [88]. In turn, the hypofunction of the HPA axis has been hypothesized to trigger the behavioral and physical changes linked to domestication ([89]; more on this in Section-6). The hedonistic function seems to underlie other functions, because enjoying musical sounds generally makes a human listener less prone to engage in aggressive behaviors, thereby promoting social interaction, conducive for the emergence of higher order functions.

The ontogenetic line of acquisition of musical skills, perhaps, provides the most instrumental source for the reconstruction of the earliest stages of phylogeny of music - especially, the genesis of the divergence between verbal and musical modes of expression [29]. We expect these patterns of ontogenetic development to apply to phylogenetic development. The transformation of protomusic into music must have followed the ontogenetic principles. After all, cultural evolution is directly formed through the ontogeny: cultural phenomena can exist in no other way but through the transfer from one generation to another, where a collective of people is always a sum of individuals. A society cannot sing a song. Only an individual can sing a song. And every generational transfer requires an adult expert catering to the psychophysiological limitations of an infant. Thereby, the phylogenetic conservation as a rule relies on the ontogeny [29].

Hence, the musical functions that find their counterparts in speech functions are likely to precede the emergence of those functions that are specific to music. The earliest ontogenetic musical behaviors appear shortly after the birth as interpersonal forms of musicking that teach an infant to be receptive to and distinguish between the stimulating and relaxing musical stimuli, projected by adult communicators through expressive vocalizations, gestures, mimics, touch, and interactive motion (like manually moving the infant's legs to the rhythm of vocalizations) [90]. Learning how to communicate, following the directions suggested by pleasant stimulation, seems to constitute the evolutionary important consequence of interpersonal bonding at the onset of the acquisition of music skills [91]. The infant does not yet engage in active musicking - the only "musical" feature voluntarily produced by him is the harmonic timbral coloration: phonation of resonant frequencies in the vocal folds that emerges at about 2 months of age [92]. However, the cross-modal correspondences between auditory, visual, haptic and locomotive experiences, induced by caretakers, along with child's innate impulses, activate child's "knowledge instinct" - i.e., an internal drive to improve the knowledge of the surroundings [93]. On par with the instincts for food and procreation, this instinct bears immense evolutionary importance across higher mammals, increasing long-term chances for their survival, which justifies short-term risks of curious behaviors (injury or even death). And "epistemic curiosity" [94] aligns very well with music. 
Curiosity rewards sensory change and enhances memory [95] - in line with the mnemonic (conservation function) and the diversifying (recreation function) uses of music.

The learning aid function interacts with the interpersonal bonding function throughout the first year of life, preparing the next function - recreation. The latter is manifested in "musical babbling" [see 96-99] and self-entertaining exploration of the expressive capacities of vocables. These activities are the earliest active forms of musicking, although they are poorly differentiated from verbal vocalizations. Their phylogenetic analogs should be regarded as organic parts of "musilanguage" - a paradigm of a common evolutionary precursor of music and language [2].

Developmentally, music grows out of musical babbling that is characterized by wandering pitch and stretchable melodic intervals. Babbling gradually becomes accompanied by physical movement of increasing variety, involving feet, head, and hands, which become coordinated through synchronization of physical motion to melodic "motion." This coordination grows to become consistent throughout the second year of life, after which it seizes to improve. As noted by Holahan [98], at first, children do not babble for any other reason than musical stimulation, and tend to join discrete musical elements in attempt to synchronously follow the salient features of external musical stimuli (they do not aim to generate tonal organization on their own). Later, children notice and explore expressive capacities of their utterances, initially to the external music and thereafter without it, completely on their own - but such musicking does not give rise to patterning of pitch or rhythm. Finally, babbling becomes more coherent and only partly resembles (but is not identical to) the musical features of familiar songs. At this level, the first signs of a recurring "pitch center" and a consistent tempo appear, indicating that children become aware of the relationships between the successive sounds of a melody and the rate of these sounds' change.

From this point on, music development diverges from speech development, and music functions start contrasting speech functions more and more. Verbal babbling starts conveying referential information in a dialogic format with a caretaker (but often involving solitary impersonation of an imaginary interlocutor), whereas musical babbling mostly conveys affective information in a selfdirected monologic format, entertaining the child [29]. The recreation function (No.7 III of Fig.2) and its two derivative functions, physical aid, and emotional communication, can still be carried out by verbal communication. Like music, speech can convey emotions, entertain (onomatopoeic imitations, tongue twisters, nurseries), and accompany physical activities (play-games, work) although not as effectively as music. For this reason, it is hard to delineate, which exact uses belong to the musilanguage and which - to the fully-fledged music. We shall try to draft a plausible order of music functions following the sociocultural approach to the mental development by Vygotsky: by establishing which function becomes a "central neoformation" (i.e., formation of a new mode of interaction with cultural environment that requires the emergence of new psychological function) [3] in each of the developmental phases in acquisition of musical skills.

The beginnings of music development are characterized by syncretic co-dependence of functions. The bonding function relies on the hedonistic and learning functions, while determining the recreation function: an infant acquires the capacity to babble and have fun by mimicking and exploring the sounds and articulations learned from caretakers during interpersonal communication. The recreation function, in turn, determines the physical aid function and the emotional communication function: a child figures out which form of activity corresponds to a music pattern, based on the experience of recreational exploration of sounds. The realization that music can reveal one's emotional state comes from multiple observations of the correspondence 
between the improvised patterns of sounds and the concurrent affective state. The latter also directly relies on the experience of interpersonal bonding, because musical emotion organically relates to emotional contagion, which requires the presence of at least two affiliated parties. Emotional regulation (No.2 IV.B) also is syncretic: it requires emotional communication via musical emotions, experience of interpersonal bonding through music, and knowledge of a repertory of basic musical patterns, reserved for expression of certain musical emotions. This knowledge is acquired through the physical aid function (No.9 IV.A), which puts in place the system of musical genres - a child learns which musical patterns match characteristic forms of locomotion in games with other children and solitary playing with toys, construction games, and drawing. They all typically involve impersonation of imaginary characters through singing or motherese-like talking.

Three earliest functions, enclosed in the blue rectangle in Fig.2, seem to be genetically programmed and common not only across human music cultures, but across numerous social animals that possess a system of co-specific vocal communication [100]. These functions are most actively exploited by human infants during their first year of life. The subsequent 5 functions (marked by green color) are usually mastered through the 2 nd to 4 th years of life. The babbling stage (recreation function, phase-III), described above, leads to the exploration of play-singing (physical aid function) to accompany child's motions in a game and to impersonate characters represented in games, drawings, or phantasies [97,101,102]. In parallel, starting from the age of two years, children engage in spontaneous creation of brief original non-imitative songs based on pitch contours of indefinite pitch - such songs are usually private (children often stop singing at presence of others) and retain a specific thematic material, performed for a period of a few weeks and renewed afterwards [96,97,103,104]. These songs introduce the active use of the function of emotional communication, at first self-directed and shortly later directed at surrounding people $[97,105,106]$. The main incentive of making a song here seems to be an impulse to come in touch with personal feelings and fix an impression of the encounter with a world in an attempt to understand it [106].

The discovery of musical emotions in self-communicated songs and the recognition of music genres through child's observation of correspondences between certain physical activities and music patterns constitute the main achievement of phase-IV. It opens the gates to conscious manipulation of one's affective state solely by musical means, without conducting the corresponding physical activities. Mastering of emotional regulation occurs at phase-V. About the age of 3-4 years, children acquire the ability to recognize musical emotions of sadness and happiness in music they listen to [107-109]. Four-year-olds can intentionally express musical emotions in their own original songs [110-112]. At around the same age, children start using music to deliberately regulate their emotional state to their liking [113]. This 5th phase helps growing children meet their main challenge in emotional development - the necessity to adjust their individualistic impulses and demands on their caretakers to the interests of other members of their immediate social environment. This poses the need to comply with behavioral norms of a growing circle of people (at first family and friends, then the preschool staff, the kindergarten, with their peers and their relatives, etc.) [3,114-116]. In most folk traditions of pre-industrial societies, genres of children's music satisfy their primary objective to promote a smooth transition for assimilation into the adult culture, where adherence to the rules of active music-making serves as a prototype of adherence to the rules of social behavior [117]. 
Phase-VI introduces the last of the functions that characterize the acquisition of musical skills by a normal preschool child. The importance of this point is that those functions that are required for normal musical development in early childhood are likely to be cross-cultural and even universal, whereas the functions that follow phase- 6 are likely to be culture-specific. The earlier a function is positioned in a timeline of music acquisition, the more it is controlled by the biologically ingrained mechanisms of processing music, and the later it is positioned, the more it depends on learning and culture. ${ }^{18}$ Newborns across the globe sound more or less the same in their narrow repertory of cries and coos [119]. In contrast, music created by 4-8-year-olds bears footprints of their native music culture and therefore considerably varies between different cultures [117].

- The first 3 functions (phases I-II, marked blue in Fig.2) probably constitute universal traits of music and share genetic roots; they correspond to the musilanguage stage in the evolution of music.

- The next group of 5 functions (phases III-VI, marked green) are cross-cultural and rely partially on biologically ingrained mechanisms of processing music and partially on cultural exposure; phases III-IV correspond to the proto-music stage, whereas V-VI - to the transition from proto-music to music.

- The last group of 6 functions (phases VII-X, marked yellow) are culture-specific (some of these functions can be entirely missing in a particular culture) and represent the cultural evolution of music.

Phase-VII is intermediary between the "genetically controlled" and encultured functions. In traditional music of indigenous people of Northern hemisphere, the institute of personal song is the clearest example of the coalition status function - it displays the kin, ethnicity, and place of origin as an inter-ethnic "identity badge" of a song owner [12]. The obvious signs of decline and disappearance of personal song in regions where it used to be functional suggest that the coalition status function has been fundamentally transformed from active use by all members of a traditional society into passive use, characteristic for Western industrial societies. This change must have accompanied the transition from traditional nomadic lifestyles to Western lifestyle. This transformation removes the conserving taboos that protect the institute of personal song (its "honest" use and cross-cultural intelligibility) and turn personal song into just a regular song that is supposed to be appreciated like other forms of music. This process was observed during the end of the 20th century in Chukotka [120]. In Western music culture, the coalition status function takes a form of public exposure of one's preference for that kind of music whose public image complements one's social identity and social ambitions.

Phase-VIII is bifurcated into two parallel functions. Coalition status display enables both: conservation of memories and self-promotion through music. The former is already evident in the tradition of personal song - the custom of singing personal melodies of the beloved relatives, when they are away, to alleviate nostalgia [12]. Singing one's own personal song continuously during the critical time of solitary struggle for survival (snowstorm during one's travel) in order to maintain the presence of spirit and self-awareness also constitutes a mnemonic function. On the other hand, the personal song is linked to the function of self-promotion as well: personal melodies are still often used in Siberia and Russian Far East in boasting songs of males (e.g., "drinking

\footnotetext{
${ }^{18}$ Lev Vygotsky and Aleksandr Luria dedicated years of studying mono- and dizygotic twins (150 pairs of the ages 614 ) to establish the cut-off age at which child's higher psychological functions (including music) become relatively free from "genetic control" and concluded that this typically occurs within the range between 7-12 years of age [106 p.313314]. This conclusion generally agrees with the ethnomusicological observations that the earliest original songs created by children share many common features across the world's cultures [118].
} 
songs" that usually praise one's strength and smartness) and in autobiographical songs of females (listing their achievements in supporting the family) [121,122].

Both functions of phase-VIII also have passed through fundamental transformation that accompanied the socio-economic changes throughout human history. Conservation of memories has become predominantly passive - taking the shape of patronizing a specific type of music and specific songs related to one's nostalgic memories. This use has lost the semiotic component that was present in personal songs, replacing it with subjective personal and interpersonal aesthetic appreciation (within a small group of close people), based not on exploiting specific structural properties of music but on idiosyncratic past experiences. In contrast, self-promotion retained its active format of presentation but became exclusive - employed by only those individuals who had discovered their special musical aptitudes and capitalized on them by learning impressive technical skills, sufficient to draw people's admiration. This use also suffered semiotic deterioration, since the success of technical show-offs does not rely on the audience's understanding of musical structures.

Each of two functions of phase-VIII receives its derivative in phase-IX. Conservation of memories puts in place an ability to conserve a reference to a particular event, place, or person and to retrieve this reference, whenever a referent is absent during the music act. Thereby, the listener obtains the capacity to contemplate an event at personal convenience. In Western classical music, this function carries the heaviest semiotic load, most obvious in programme music. On the other hand, the function of personal profiting clearly evolves from the function of self-promotion: those exceptional musicians who obtain recognition for their music skills can benefit from their reputation. However, this function activates an economic incentive to reduce conventionality of music expression and, therefore, reduces the semiotic efficacy of music. Personal profiting triggers competition between music-creators for monetizing their skills, which promotes originality and innovation, while encouraging showmanship of the style rather than success in expressing a chosen theme. This tendency often supports mannerist forms of music that can eventually lose their semiotic ground and become essentially meaningless (meaning anything the listener wants to hear), as it happened in the transformation of modernist music into postmodernist [16].

Finally, the calling to action function of phase-X emerges from the function of contemplating an event. Certain musical idioms (e.g., fanfare) obtain the capacity to signal specific actions and become adopted as "commands" in specific applications (e.g., military calls), because of their widespread adoption [123].

As the examination of Fig.2 indicates, there is a substantial difference between the earliest and the latest functions, as well as between the traditional and the modern uses of these functions. These differences are to blame for frequent disagreements between scholars of the evolution of music. Thus, returning to the controversy that we started with, Savage et al. [10] seem to focus on phases IV-IX, when they try to define music, whereas Mehr et al. [9] - on phases I-VI. It is easy to see that their approaches only partially overlap (phases IV-VI). Overall, the discrepancies between those functions they choose to consider exceed their commonalities. And indeed, it is hardly possible to define a common ground between the earliest and the latest phases, if to take in consideration only musical behaviors without musical structures. Even the very foundation of music - the hedonistic function - is hard to spot in such modern genres as death rock, unless we look into the euphonic aspects of their characteristic features of meter, rhythm, and melody. 
The problem is that the social orientation of phases VI-X enables drastic transformation of those uses that characterize the personally oriented phases I-V. Let us take the example of children's music. In traditional folk music, this function is all-inclusive, actively exercised by all children in a community. Children's music is not separated from the music of grownups into a special niche children use adult's musical lexicon to prepare themselves for adult music activities [117]. In modern Western societies, children's music is isolated from adult music and uses a different lexicon (e.g., nursery rhymes can be easily distinguished from art songs). Access to the adult lexicon is usually available to only those children whose musical aptitudes are recognized by teachers, parents, and peers as exceeding the conventional "norms" and therefore deserving musical training [110]. Such children perform adult's music, following the same criteria as adult performers. Paradoxically, the majority of Western children abandon the practice of spontaneous active vocal music-making that they carry on throughout their early childhood. This usually occurs during middle school, due to negative self-esteem, so that their music use becomes strictly passive. In essence, Western school-age children voluntarily restrain their music communication, making it one-sided - oriented towards personal consumption. ${ }^{19}$ This is in complete reversal of the situation in traditional folk music, where children start their music development from personal use and learn to actively communicate through music, mastering its social use.

Evidently, any comparative study of music, especially in the evolutionary perspective, should not rely on functions and uses of music alone - their analysis makes sense only in conjunction with the comparative analysis of music structures. Only then it becomes possible to secure the comparison of apples to apples and oranges to oranges in looking into how specific music patterns are systemically used to convey information.

\section{The effects of cultural transmission on music structures}

Yet another issue that often causes confusion is the relative structural instability of music idioms. Even the most discrete of music structures that distinguish music from speech - pitch-classes of melody and harmony and rhythm-classes of metric organization - feature high variability in performance practice. Performers routinely engage in "expressive timing": i.e., they exaggerate rhythmic contrasts to increase rhythmic expression and facilitate recognition of rhythmic idioms, extending or contracting rhythmic values depending on the tempo, articulation, and the stylistic context [125]. As common is the use of "expressive intonation": ${ }^{20}$ i.e., sharpening or flattening the

\footnotetext{
19 It will not be an exaggeration to conclude that, recently, personal forms of consumption of music have exceeded social forms of using music globally. The most recent report of the International Federation of the Phonographic Industry states that $86 \%$ of consumers listen to music through on-demand digital streaming, $75 \%$ use smartphones to listen to music, $66 \%$ typically listen to music in the car while driving, $40 \%$ listen to music while working or studying, $54 \%$ while cooking and cleaning, and $19 \%$ when going to sleep [124]. All of these uses are non-social. In contrast, only $36 \%$ of consumers typically listen to music at concerts, gigs, and festivals. Notably, $50 \%$ of the younger audience, 16-24 years of age, choose audio streaming if there were only one way to listen to music. Obviously, this trend originates from music cultures of industrial societies and is foreign to traditional indigenous forms of music.

20 The term "intonation" is used differently by linguists and musicologists. The former use it in reference to the prosody of speech - the characteristic rise and fall of a pitch level in pronunciation of phrases and sentences. The latter refer to the method of treating a musical pitch in performance, which encompasses both, the theoretical aspect (e.g., the system of tuning) and the practical aspect (accuracy of playing or singing in tune and the extent of expressive exaggerations) [126]. Yavorsky forged yet another understanding of "musical intonation" in his framework of "musical speech". He defined intonation as "the "elementary unit of music structure that binds its semantic content to similar verbal intonation" [41]. Asafyev further advanced the "intonation theory" by conceptualizing "intonation" [Rus. "intonatsiya"] as a "tonecell" that presented a dyadic melodic interval characterized by 3 parameters: intervallic distance, melodic direction and
} 
normative pitch value in order to emphasize tension and relaxation, to shade and color melody, or to produce a better sounding chord [34]. The extent of exaggeration is governed by public conventions and is directly related to the intensity of musical emotions. Performance of music in a strictly accurate manner, without any inflections, is universally perceived as automatic, nonhuman, and substandard - especially in emotionally charged genres of music (e.g., lamentation or hymn).

Lack of documentation on rules of expressive timing and intonation has led some scholars to doubt that music possesses syntactic organization at all and to believe that discrete elements of music are defined arbitrarily by performers based on their individual liking. The falsehood of such beliefs is revealed by the presence of codified rules and the literature on appropriateness and inappropriateness of exceptions from these rules in those music cultures that have developed a formal music theory (e.g., Western classical music, maqam, raga). Many folk cultures, especially of populous ethnicities, are known to possess implicit music theory that is orally taught by mastermusicians to their pupils. However, the rules of such unfixed theories are subjected to diachronic transformation that can occur quite fast. Inherent volatility of musical grammars raises yet another crucial issue - the formative power of cultural transmission on music structures.

\subsection{The overview of the recent experimental research}

Pioneered in the field of evolutionary linguistics, research on cultural transmission has unveiled that the practice of multi-generational learning and communication directly affects linguistic structures. Recent experimental studies on artificial language learning, comparative animal communication, and experimental semiotics have forged new methods of identifying and measuring the contribution of cultural transmission to the structural features of languages. As a result, the traditional linguistic disciplines of phonology, morphology, and syntax became expanded to cover the study of artificial languages and animal communication, which brought to life a new field of study: of combinatoriality and compositionality. It was conceptually formulated by Charles Hockett in 1960 as part of his theory of duality of patterning [127]. Studies on combinatoriality focus on how and why combinations of meaningless elementary units (e.g., phonemes of natural languages) can generate meaningful morpho-syntactic units (morphemes and words). Studies on compositionality focus on more complex levels of syntactic organization (e.g., principles of generation of sentences).

Combinatoriality and compositionality characterize human communication. Very few animal species combine different signals in a single compound, which occurs in the most basic form. Building blocks, used by animals repeatedly to construct a call, do not carry semantic power, so that the rearrangement of blocks does not change the meaning of a call [128]. Only humans were able to take advantage of the capacity of combinatorial communication to use fewer elements to express the same number of messages as non-combinatorial systems. This is because only humans can reliably express and recognize communicative and informative intentions. Linguistic meaning is inferred based on the listener's assumptions of the speaker's intentions even in the absence of the object of reference in the speaker's immediate environment. We are the only species capable of this. For other species, the major obstacle for developing combinatoriality is the holistic nature of their signaling. A single piece of information is conveyed by a single call in its entirety and

relative euphony [7 p.289]. It is this understanding of "musical intonation" as a semiotic sign forged in a community of music-users that was adopted by musicologists of countries of the former Soviet bloc and became the cornerstone of the musicological intonational analysis of all forms of music [8]. 
situations where the triggers for signaling are composite are rare - i.e., compound relations between the call's referents cannot trigger calls that would have compound relations [128]. This is in contrast to humans, whose communication can naturally evolve from non-combinatorial to combinatorial, based on natural selection for optimal acoustic distinctiveness [129]. It was experimentally demonstrated that initially continuous series of slide-whistle sounds were eventually broken into discrete recombinable elements as a result of being taught and reproduced through a multi-user transmission chain [130]. Especially illuminating are the studies of the emergence of village sign languages. They reveal that the combinatoriality of the signs (e.g. different distinctive features, like hand shape, are combined in diverse ways to generate a multiplicity of signs) develops gradually as the volume of input increases, the community grows in number, and signers interact more frequently and in more diverse contexts [131,132].

Cultural transmission of linguistic structures promotes compressible representations. Each participant in a transmission chain tends to intuitively strive to minimize entropy while learning a structure, which has cumulative effect at the end of the chain. Lower entropy enables compression of information, which in turn, paves the road for the emergence of compressible regularities, generating grammars [133]. The compromise between compressibility and expressivity determines the genesis of compositional organization: novice learners promote compression of linguistic structures to simplify learning, whereas master speakers promote expressivity to increase the impact of their communication [134]. Another transmission-related factor that contributes to generation of grammars is the regularization of variants - each instance of learning tends to introduce a modification aimed to uniform those structures that are just slightly different [135].

The same factors that determine linguistic transmission seem to be at work in transmission of music, as it follows from our review of functions and structural features that distinguish music from speech - at least for its earliest ontogenetic functions and for the aspects of pitch, rhythm, dynamics, and timbre that are shared with language. Moreover, the greater capacity of music to secure entrainment and emotional contagion should promote more effective communication as compared to languages, and the greater mnemonic power of music should support more effective learning. The balance of power between the communication and learning capacities of music is likely to depend on the number of people engaged in a music act - a variable that is practically absent in verbal communication. ${ }^{21}$ The greater the number of participants in a music act, the more important a transmitter of information becomes as opposed to a receiver, prioritizing expression over learnability. The reverse is true for the smaller number of participants. At the extreme, solo musicking "for-oneself" virtually removes the concern for the impact of expression. Mother's singing and motherese, prototypical for the interpersonal bonding function, focus on the receiver's end of communication, prioritizing learnability over expression.

This distinction is very important for structuring music idioms. Musicking for and within a large group of people encourages a performer to employ musical rhetoric - to intuitively strive to stir the listeners, which promotes intensification of emotional display and innovation of musical means of expression, in the long run accelerating the evolution of tonal organization of music [136]. In contrast, musicking for oneself and for another person promotes conservation of musical means of expression - a music-maker does not have an incentive to exaggerate the expression for a single well-familiar listener (usually a family member) and is likely to stick to the "good old" idioms

\footnotetext{
${ }^{21}$ In oral languages, person-to-person communication overwhelmingly dominates over group communication. That said, there is evidence (briefly mentioned in section 7) that semantic compositionality is greater in languages spoken by larger human groups.
} 
appropriate for a particular expression. The same formula is accurately reproduced in every act of music transmission. High conservation usually freezes the evolution of tonal organization. Prevalence of solo- and duet-musicking in a music culture might be the reason behind greater preservation of native music tradition in comparison to native tongue, noted by some ethnomusicologists $[65,137,138]$. Greater mnemonic power of music, evident in a purely musical phenomenon of earworms, is likely to secure the advantage of music.

The dividing line between the transmitter-oriented and receiver-oriented music seems to lie somewhere around 4-5 participants. It is closely related to the issues of critical distance of hearing music: like language, timbre-oriented music requires close distance and therefore is naturally limited to a few individuals who can fit in a spot within a few meters around a music-maker, whereas pitch-oriented music affords much larger distances [12] - up to a few kilometers, as in such genres as forest calls of gatherers, pastoral calls of herders, and "talking drum" music.

Recent experimental studies on transmission of music confirm that the transmission has power to alter pitch and rhythm aspects of the transmitted pattern. Thus, a study arranged in the format of a multi-generational signaling game demonstrated an increasing diatonization of the initially chromatic melodic model [139]. This transformation seems to have originated from the pressure to regularize a hard-to-grasp chromatic organization on the part of those receivers whose information-processing bottleneck was smaller. They tended to simplify the intervallic typology of the music they heard. Therefore, the entire transmission chain became structurally shaped by the participants who had the least developed ear. This conclusion of the authors of this study agrees with the observations of musicologists that indigenous folk music tends to contain simpler forms of tonal organization in comparison to "art music" of cultures that possess codified music theory, formal music education, and music notation [140]. The underlying cause must be that folk music relies on person-to-person transmission and implicit learning (very much like the settings in the above-mentioned study [139]), which are more vulnerable to constraining effects of the brain to process the auditory data. Music notation and formal training provide an additional resource for overcoming the natural limits of one's music hearing.

\subsection{Cultural bias in oral transmission of music}

Oral transmission cultivates different intonations in comparison to transmission of notated music - even within the very same geographic region. For example, according to the statistical analysis of the melodic structures of the corpus of folk English and Irish tunes and experimental multigenerational study, the phrasal cadences of Western folk music tend to use the descending step towards the tonic, whereas in Western classical music cadences, the tonic is usually reached by the ascending half-step [141]. The commonality of the latter is even reflected by the nickname of the VII degree in a diatonic scale - the "leading tone" - since it "leads" to the upper tonic. One of the leading Russian analysts of music, Leo Mazel, also noted that the "leading intonation", so characteristic for classical tradition, is quite rare in folk songs of Slavic peoples, as well as in Middle and Near Eastern melodies [142]. The origin for this discrepancy seems to come from the influence of the Ancient Greek music system that was based on the descending melodic standard, reflecting the consensus that ascending steps accumulated tension, whereas descending steps relaxation [143]. This convention is still observable in music of Persian, Arabic, and Andalusian traditions. It is documented in the tradition of Byzantine chant: although the Octoechos music system allows the "leading" tone to ascend to a more stable tone, more commonly phrases are terminated by a descending 2nd, in a "Phrygian" style (A-G-F-E) [144]. Such an ending was 
considered more appropriate for "Godly" music compared to the disturbance of the ascending motion. The use of the semitonal VII degree is a great rarity in the majority of known ecclesiastic melodies. The descending standard of European and Western Asian traditions might constitute the Greek heritage of the Byzantine culture, reinforced by the authority of the Church, and adopted by local secular traditions.

The example above is just one of many examples of cultural bias - i.e., a tendency of individuals and social groups to prefer a specific structural feature over another due to the ease of its recognition, its ubiquity in a native cultural tradition, and/or social prestige associated with it [145]. The first of these factors can be biologically ingrained: brain circuitry makes it is easier to process consonant harmony than dissonant, melodic steps than leaps, regular rhythms than irregular, binary meters than non-binary, tempo whose rate is matched to one's heart rate than other tempi. But learned distinctions can also be easier to process - e.g., nearly automatic recognition of familiar musical modes, such as minor vs. major. The other two factors are strictly cultural. All 3 factors are engaged in transmission of music, more important for a receiver. The best demonstration of this is the practice of transcribing folk tunes by ethnomusicologists. Despite all training and special notation signs reserved for reflecting the nuances of pitch and time organization, hardly two ethnomusicologists transcribe the very same music they witness or the same sound recording in the same way - the pitch and rhythm values, as well as metric organization is nearly always represented differently by each of the transcribers [146]. And even specific training to find the best transcription options does not guarantee the uniformity of transcription [147]. Not only do transcribers diverge in their interpretation of what they hear, their transcriptions often distort the actual tonal organization in the transcribed music. Thus, the software-based frequency analysis of transcribed archive field recordings of Lithuanian traditional songs, followed by the statistical analysis of frequency values, revealed that many of the notated chromatic alterations were not justified and presented, in fact, an exaggeration of expressive diatonic intonation of the performers $[148] .^{22}$

In a similar vein, all published transcriptions of the non-imitative music vocalizations by infants are fundamentally distorted by being represented in the form of notation of Western classical music or by graphic visualization based on the equal temperament [29]. Obviously, such interpretations are bound to be erroneous, since children develop the ability to recognize pitch-classes as members of specific pitch-class sets not any earlier than by the age of 3-4 years. Prior to this age, children must perceive tonal organization following a scheme that is different from Western tonality (that they have no means of hearing) - even if they are exposed to Western music in their cultural environment. Transcribers of children-made music introduce their cultural bias in the transmission chain: their distortion can feed the next round of transmission, if one of their transcribed tunes is published in a collection of music for children, and preschool teachers start teaching this published version to children in their daycare.

Cultural biases are not limited to Western interpreters of non-Western and children-made music. Thus, traditional Turkish musicians face the same challenge when interpreting intervallic values in the makam music [149]. Even cultures that do not possess a formal music theory, and therefore lack standards for defining pitch-classes, are subjected to biased frequency resolution. Alekseyev demonstrated systemic distortions in the Russian-Ukrainian song "Provody", originally created in a diatonic minor heptatonic mode, when this song was reproduced by Buryat and Yakut traditional

\footnotetext{
22 Notating a sound as an alteration of a normative pitch class implies that the performer recognizes that sound as an inflection of a pitch-class of a musical mode as opposed to regarding that sound as a pitch-class of its own.
} 
musicians [136]. The Buryat version converted the half-tone interval-classes into whole-tone, thereby transforming a song into anhemitonic pentatonic, in accordance with the pentatonic standards of Buryat music. The Yakut version also modified half-tone intervals, but in a different way - by converting the very same melody in the anhemitonic tetratonic mode. Biases like that are responsible for what can be termed "musical accent" [150]. Like a sociolinguistic accent, musical accent allows native music-users to identify "foreigners" that attempt to use their music system. And just like speaker's accent rises from the competition between two phonemic systems, when the second language has been acquired later in life, so that its phonemes are mapped onto the phonetic system of the native language rather than in parallel to it, musical accent emerges in those individuals who learned a foreign music system past early childhood [151]. Musical "accent" constitutes a cultural bias that has the power to skew the entire lineage of cultural transmission towards the systematic transformation of pitch- and interval-classes.

What is important, cultural bias is experimentally found to introduce tonal organization into totally stochastic models, affecting not only the pitch aspect but also music form and articulation. The continuous glissando melodic shapes, generated on slide-whistles and passed through the transmission chain, became "rasterized" into discrete pitch values, forming sets of less than 7 pitch-classes per each participant, obtaining motivic patterns, evened up in the trajectory of their shape, and broken in shorter phrases $(<9 \mathrm{sec})$ [152]. Similar regularizing effects were observed in the domain of rhythm: after just several transmissions, initial random combinations of various durations acquired structurally similar patterns, combined into rhythmic figures (the authors call them "rhythmic riffs"), and "quantized" into rhythm-classes ("durational categories") that eventually became isochronous, related by small-integer ratios [153]. Interestingly, the ordering effect was more pronounced, when transmissions were performed by the same individual reproducing the playback of his own prior performance, but at the cost of lower compliance to small-integer ratios - i.e., person-to-person transmission tended to average each person's individual preferences of idiosyncratic ratios [154]. This distinction agrees with the musicological descriptions of musical babbling (see above), which is characterized by prolonged solitary improvisatory reproductions of the same pattern. It is plausible to conclude that music cultures, where personal solitary forms of musicking occupy an important place, are likely to possess idiosyncratic rhythmic divisions and metric organization - in contrast to music cultures characterized by collective music-making. This conclusion finds support in the comparative analysis of "personal music" of different Siberian ethnoses, many of which can be recognized by a peculiar rhythmo-metric organization $[136,138,155]$.

The experimental demonstration of formative power of chain transmission might project the impression that music could have emerged from random vocalizations by chance, as a result of the series of "random mutations." However, the probability of this is rather low. We should remember that none of the subjects in the above-cited experimental studies were free from cultural bias virtually anyone had been raised in some music culture and therefore obtained a native musical "accent." Musical accents make their owners "rasterize" and "quantize" musical sounds in terms of familiar pitch- and rhythm-classes. Exceptions to this are few: the newborns and the deaf. But it is technically very challenging to conduct the transmission experiments on them. Judging by the ontogenesis of music skills, ongoing random vocalizations characterize the babbling stage, but this stage follows the musical interaction with the caretakers, which is culture-based. Motherese and parental singing direct infant's creativity towards exploration of expressive means found in parental music culture. Even during the musilanguage stage, when no music prototypes were around, mother's vocalizations must have set the direction for infant's experiments with sounds, 
so the variations in infant's babbling were doubtfully random. It seems more likely that in the absence of cultural tradition, sometime in distant Paleolithic past, structural changes in the transmission chain of musilanguage were determined by widely adopted proxies of social scripts and tool-making technologies, which gradually put in place meaningful patterns for each of aspects of expression of what was later to become "music" [13].

Here we have to underline once again the role of semantics in shaping structural features of sounds through transmission chains. The type of information transferred through a chain and the format of transmission both influence structural transformations. The very same subjects in the same chain generate different structures from the same set of novel vocalizations, when asked to convey referential versus emotional information - in the first case the end result resembles speech, whereas in the second case - music, and their divergence is cross-culturally marked right from the start of a chain [156]. The cross-cultural stability of this divergence suggests that there is more to it than mere introduction of cultural bias in the interpretation of the heard vocalizations on part of the subjects of the experimental study. After all, it is the function of use that is known to shape structures of languages and music systems. And the experimental evidence confirms that artificial rhythmic progressions set to refer to emotions increase structural regularity and isochronicity while maintaining one-to-one mapping to meanings, when passed through the transmission chain [157] - so as the artificial melodic progressions mapped to emotional meanings [139]. In both cases, the regularization is determined by the individuals more limited in their capacity of auditory analysis and memorization.

\subsection{The role of formats of transmission in genesis of music idioms}

The format of transmission is also instrumental in shaping music structures. The person-to-person format, adopted by experimental studies of transmission, is not the only one. Bruno Nettl lists 4 types of transmission, each of which is characterized by its own incentive and the extent of divergence from the original [66]:

1. "Linear" - person to person - an incentive to accurately reproduce a model;

2. "Curvilinear" - group to person - an incentive to resolve the discrepancies between multiple users and fix an ideal (optimal) version;

3. "Tree-like" - person to group - an incentive to explore modifications of a model;

4. "Double-tree" (Nettl engages a metaphor of a tree infested by a creeping plant to refer to the situation where receivers merge the structures of a model with the structures of some other similar tune, popular in their community) - group to group - an incentive to adapt a model to a preferred genre/style.

To these 4 types, we should add the 5th type - "self-transmission," as in musical babbling and prolonged episodes of solitary musicking (of herders or travelers). Such activities involve transmission of the same musical material (theme) over the repeated acts of self-communication. Following Nettl's paradigm of naming, this type might be called "circular", since the transmission here starts and finishes at the same place, and the information keeps circulating within the closed system. A music-maker here has an incentive to maximize modifications of the initial model to entertain oneself. Our second adjustment of Nettl's classification will be the renaming of his type2 into "inverted tree", since it inverts the transmission scheme of type-3, and their re-ordering, because "inverted tree" is derivative of the "tree" structure (i.e., without "tree-like" variations 
already in place, resolution of discrepancies is not needed). So, our revised classification will look like this:

1. Circular (1 person, i.e., "soliloquy") - "creative": maximal variation to avoid the boredom. A music-maker keeps experimenting with an interesting musical idea, exploring expressive capacities of a musical instrument or a phonation type, or adjusting a favorite tune or personal song to accompany various daily activities.

2. Linear (2 persons, i.e., "dialogue") - "preserving": minimal variation to secure effective communication. A person engages into duetic musical communication with another person, following the format of a verbal conversation, by taking turns, where one party either mimics another party (echo format) or continues the same musical idea (verse format - i.e., delegating different verses of the same song to different performers).

3. Tree (1 person to a group, i.e., "responsorial") - "optimizing": medium variation to enhance a model. This is the most common type in folk music, usually triggered when a prototype proves to be too idiosyncratic and complex (manneristic) for a reproducer. Then the performer looks for ways to replace the idiosyncratic traits with conventional. If an adjustment proves to be idiosyncratic, too, it becomes a target for further tweaking by the next performer - until the tune becomes "polished", i.e., averaged enough to satisfy the majority of music-users within a community. Once this point is reached, the transmission transforms into the inverted tree type (see below).

4. Inverted tree (group to 1 person, i.e., "soloist") - "corrective": small variation to resolve discrepancies. This type counter-balances the "tree" type in a chain of "averaging" an attractive melody to make it more attractive for everyone in a community of music-users akin to morphing of photographic images [158]. The origin of the "beauty of the average" effect lies in the processing ease, specifically in processing fluency [159], and is shown to occur in the domain of pitch and timbre [160]. Once a tune is effectively "averaged", it usually becomes conserved in its optimal form as a model for imitation (linear transmission). The inverted tree transmission is often required in religious applications of music, where a variety of people is supposed to faithfully reproduce a "supernaturally" important music that is expected to work only when properly rendered.

5. Hybrid tree (group to group, i.e., "antiphon") - "merging": large variation to assimilate a foreign model. This type is evoked when a model turns out to be too similar in its structural features and its semantic value to an already popular tune within a given community of musicusers. Performers half-consciously mix up two models, and the hybrid obtains life of its own (open for new transmissions). Hybridization can occur when a popular tune is imported into a different genre - in this case, it has to be adjusted to meet the standards of a new genre (e.g., "jazzing up" a famous classical music theme).

Each of these types exists within a spectrum of applications. For instance, jazz music often combines circular, tree, and hybrid tree transmission. However, the benefit of distinguishing between 5 above-mentioned transmission types is that together they permit a researcher to capture how a given musical structure can be implemented in real life. To remind, any structure must be effectively reproduced (i.e., retain its basic structural and semantic identity) through the extensive chain of multiple reproductions in order to stay alive across generations. The interaction of transmission formats, their incentives, operational functions, and typical uses makes transformation exceedingly complex, nothing like lab settings of the experimental studies of cultural transmission. We shall try to show a gist of this complexity in Fig.3 below. 


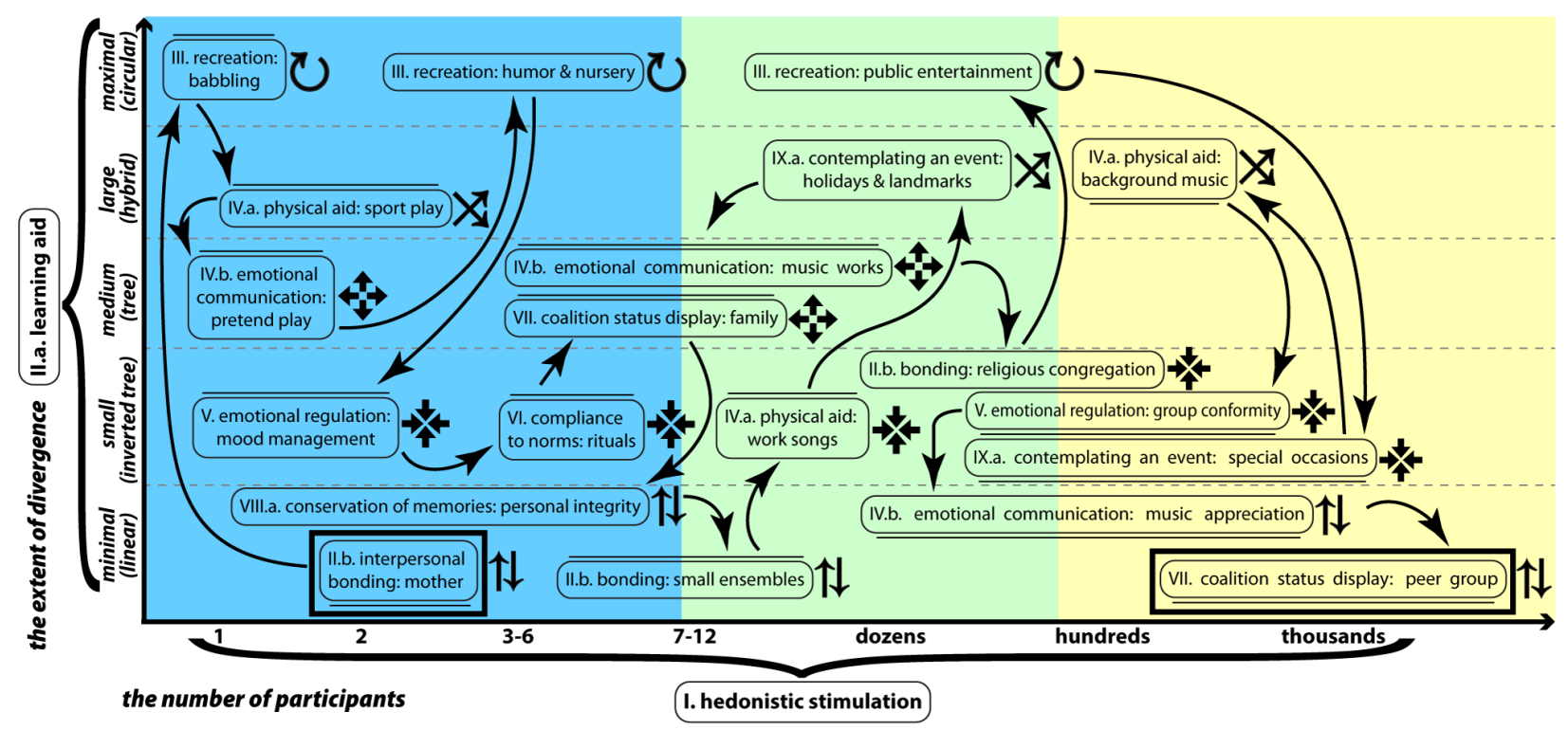

Figure-3. The example of transformation of a musical intonation through transmission in a modern non-urban society that retains folk music traditions. A thick rectangle at the bottom left marks the starting point and that at the right - the ending point of the transmission chain. The horizontal axis indicates the number of participants in each transmission act, reflecting the growing expressivity (i.e., the orientation to stimulate a receiver), required to reach greater audiences. The vertical axis indicates the extent of divergence between the offshoot versions of a transmitted intonation in each transmission act. This axis is segmented by 4 dashed lines into 5 areas representing 5 transmission types, in the order of the increasing divergence towards the top. This axis reflects the decreasing regularity. Thin rounded rectangles enclose operational functions (as identified in Fig.2), tagged with a specification of the type of use that characterizes this function at a given transmission phase. Functions are numbered according to their operational dependencies, following Fig.2. Thin arrows show which functions succeed each other. An icon at the right side of each rounded rectangle represents the transmission type. An extra line under the rectangle indicates that a function is passive (i.e., oriented towards the listener), above the rectangle - active (oriented towards the performer), and its absence shows that the function can be both passive and active. The background colors reiterate Fig. 2 by marking 3 general evolutionary stages - to reflect the extent of cultural uniformity in transformation of structural and semantic features of the transmitted musical intonation. This figure is original.

This figure tracks the transmission of a melodic intonation through almost all operational functions - except self-promotion, personal profiting, and calling to action - which is representative of the cultural experience of an average member of a present-day community that still cultivates folk music. The depicted transmission chain illustrates how a melodic structure initially generated by the mother to lull her infant eventually turns into the musical idiom for the entire music culture, including most of its members, following the theory of musical intonation by Asafyev [5-8]. The crystallization of a musical idiom involves not only the effective attempts of transmitting the initial structure and its semantic value but the ineffective attempts as well. The process of "natural selection" for a musical idiom consists of the totality of successful "adaptations" to new uses and failures to do so, followed by series of extinctions of unsustainable uses. A musical structure and its meaning are shaped both by positive and negative definitions: as what they are and what they are not - i.e., what a given musical idiom is suitable to express and what it is unsuitable for.

Fig. 3 can be thought of as the illustration of the long journey of the intonation of a sliding descending 3rd, most typical to soothing motherese and lullabies [161]. At first it turns into a motif 
of the "universal chant" of children [162], cross-culturally associated with teasing [102]. And it ends up incorporated into the theme of Ella Fitzgerald's song "A-Tisket, A-Tasket", which in 1938 hit No.1 in Billboard's sheet music, Record Buying Guide, and No.3 in Your Hit Parade charts, thereafter turning into a jazz standard. This theme expresses the cuteness of a little girl.

Audio-2. Nganasan lullaby. The series of varied reproductions of the intonation of the descending minor 3rd in glissando style. Courtesy of Oksana Dobzhanskaya. Used by permission. http://chirb.it/K9pKLn

Audio-3. This recording captures spontaneous singing of a 4-year-old girl during her playtime. The melody is identical to Hargreaves' notation of what he called "universal chant" [162]. It consists of 4 variations on the intonation of the descending 3rd, where the 1 st tone is stressed by longer duration and greater amplitude (F\#-D\#, G\#-F\#-D\#, F\#-F\#-D\#, G\#-F\#-D\#). Courtesy of Jan Sverre Knudsen. Used by permission. http://chirb.it/kLeNkG

Audio-4. Ella Fitzgerald - "A-Tisket-A-Tasket", a clip from the film "Ride 'Em Cowboy" (1942). The theme is based on the "chant". https://www.youtube.com/watch?v=1bgFkeDLpSI

The original intonation (Audio-2) serves the bonding function (IIb) between the mother and the infant, reminding the latter that she is loved and cared for. The communication is strictly linear: the mother calms the child, and the child shows satisfaction and happiness. The intonation is well conserved because the child does not actually reproduce it. Reproduction is delayed to the babbling stage (III), when an infant entertains herself by toying with the learned intonation. Circular solo variations explore the limits of transforming this intonation. The invented versions are put to accompany the physical activities of the child (IVa), at first solitary and then with a partner. Different activities, executed by the same participants to the same intonation, promote hybridization of different versions. The discrepancies between different versions become further exaggerated in solitary pretend play with toys and activities such as drawing (IVb). Different protagonists are likely to inspire the child to adapt the intonation to each new impersonation. The fun of creative modifications calls for the second round of exploration of the same intonation (IIIbis) - this time in recreational activities with family or friends, musically engaging the grownups. The most salient and memorable versions of the same intonation, forged into a motif through these activities, are thereafter tried out in new personal contexts (Audio-3). This exposes their usefulness for mood management $(\mathrm{V})$. The child discovers that this motif is affiliated with musical emotions that can be handy to reduce negative emotional experiences. This initiates the inverted tree transmission: the child gets rid of those variants that prove to be inefficient in emotional enhancement. The acquired schemes of emotional regulation are put to use in curbing the child's instincts to demand immediate satisfaction of her perceived needs (tantrums). The motif brings her emotional state in tune with the other family members and is incorporated in daily rituals (e.g., playtime) (VI). Ritualization further increases the tendency of the inverted tree transmission to filter out multiple versions. Habituation to the family rituals makes the child an active member of a family, promoting caretaking behaviors towards other members. As a result, the child develops a family identity, which includes music - this favorite "chant" (VII). The display of family status encourages the child to generate more versions of it to suit the variety of family activities.

The next link in the chain, conservation of memories (VIIIa), opens a new cycle of transmissions by terminating the family-oriented cycle and focusing on the extra-family uses of music (e.g., the preschool and kindergarten environments). The child builds a new social identity for the interactions outside of the family circle, which poses the need to revise the musical preferences. 
This activates the function of conservation of memories in order to maintain personal integrity under external pressures. Once established, this function is carried throughout one's life, enabling bonding with non-family individuals (II-bis). Both, conservation of memories and extra-family bonding, are oriented towards preservation of musical material, where the same motif is accurately reproduced throughout multiple music acts to support one's sense of "self" and of "inner circle" of the closest friends. The established notions of "I" and "we", along with their musical concomitants, open the possibility of engaging into collective musicking while doing something together. In traditional folk music cultures, the first experience of this comes through participation in the performance of work-songs (IVa-bis), since children as a rule are delegated some work. Like sport play in earlier childhood, work-songs cultivate the correspondences between music structures and specific physical activities (i.e., characteristic gestures, such as pulling, pushing, lifting, hitting, etc.). The need for their close match causes the transmission to eliminate the poor correspondences (the inverted tree type). The experience of collective work-singing enables further advance in the numerosity of participants in a music act. Celebration of holidays and special occasions occupies an important place in the social life of traditional societies, where the child can observe the musicking patterns of multiple performers and partake in some music acts (IXa). Under the influence of these patterns, the music intonations acquired earlier in life are likely to be revised and enriched. At this point, the child can comfortably meet the aesthetic standards of a folk community and incorporate a set of mastered musical intonations into creation of her own theme (IVb-bis) (Audio-4). This application takes the earlier experience of emotional communication through music to the new height of communicating to an audience, be it real or imaginary. Reproductions of the same music are bound to introduce the multiple versions (the tree type).

The next transmission cycle further expands the audience size. The second revision of the bonding function initiates this expansion by binding the adolescent with peers in school, their relatives, and friends, and gradually extending to other social hubs, such as a congregation in church (IIb-bis2). If the first bonding was about childcare, the second - about collaboration, this bonding is about ideology - oriented towards establishing the circle of similar-minded people. Religious community is the most culturally common implementation of this function. The task of conforming to the congregation's taste causes a major stylistic rearrangement of one's repertory of intonations and the discarding of stylistically undesirable versions. The selected versions are passed through the new round of creative exploration within the newly found cultural community (IIIbis-2). The theme obtains its arrangement. At this point, the active use of music starts yielding to passive consumption that characterizes modern societies. The stylistically preferred variants of intonations become filtered out again (the inverted tree type), this time by finding their closest matches in the available music market and patronizing the music works that contain these matches, as well as artists who perform these works (IXa-bis). These music works acquire associations with specific events and occasions, which semantically redefines their characteristic musical intonations. The arrangement is finalized. Once the pool of preferred music works and artists is established, the adolescent starts consuming background music throughout much of the daily activities (IVa-bis2). This use constitutes the present-day passive equivalent of the physical aid function, since the choice of music has to match the physical attributes of the activity to which music serves as a background. Musical structures typical for each of the background applications further influence the choice of musical intonations - the old preferences become redefined once again. Music styles and genres that cultivate the newly redefined preferences are thereafter used to support emotionally one's conformity to the standards of the ideologically preferred social group that might include thousands of people (V-bis). Modern technologies support interaction of millions of people via social media and personal digital devices. This use once again narrows down the range of 
aesthetically attractive intonations. Music works that contain these intonations form the base for one's music appreciation - our hypothetical adolescent is likely to retain the affinity for the appreciated types of music through adulthood (IVbis-2).

Quite long transmission chain in our example is terminated by the crystallization of a new musical idiom exemplified in a musical theme of a popular song (A-Tisket, a-tasket) that represents the musical taste of a specific social group - appreciation of this song works like a badge, wearing of which displays to the public one's membership in that group (VII-bis).

All 19 links have contributed to the structural and semantic (re)shaping of the original melodic intonation through cycles of complex transformations, starting and finishing with the linear transmission, called to conserve this intonation. The entire course of development ran like ripples in a pond caused by throwing a stone in it: spreading over the greater body of people, gradually losing momentum as the transmission distance increases. Active use of the earliest phases became superseded by passive use along the axis of increasing cultural diversity (very stereotypical crosscultural beginning and hardly predictable culture-specific end of the chain). In other words, the priority of expressivity in personal and interpersonal communication was eventually taken over by the priority of ease of perception (learnability). In parallel, the entire chain started off by the utilitarian signaling of an emotional state, but ended up by demonstrating cultural identity via aesthetic and social conventions. This transformation occurred through "recycling" of the same functions along the axis of progressive increase of the number of participants - reflected in our analysis of Fig.-3 by the use of "bis" after the function number. Seven functions were recycled this way. Each of them became transfigured by the multiplicity of participants in a music act. As a result, the entire chain unveiled itself like a nexus of spiraling functions, some of which changed their transmission typology (e.g., coalition status display switched from the tree to the linear type), whereas others did not (recreation stayed circular).

If we track the transmission chain in Fig.3 in reverse, the nature of the spiral organization becomes obvious. Status display in a peer group is possible, because an adolescent from our example wants to befriend this group and appreciate music together. The desire to join in is fueled by the love of a certain type of music. This love, in turn, emerges from the fun of collective creative exploration of music. Having fun is made possible by the realization of ideological bonding beyond family and school. Such bonding is promoted by discovery of the power of one's music performance to make listeners love the performer. This discovery occurs in celebrations of holidays and other occasions. Participation in celebrations becomes meaningful because of the experience of partnering in a work-song. Such partnering is enabled by building the bonds with collaborators, the incentive for which comes from the previous positive experience of earning a respected membership in a child's family. The commitment to overcome natural instincts to demand satisfaction of one's desires originates from the emerging liking of rituals. The liking of rituals is prompted by the positive experience from following the rules of playing games. Playing rules are adopted, because the child is attracted by the characters of toys that set the paradigm for impersonation. Pretend play is inspired by the pleasure from experiencing different modes of locomotion. The latter are tried out under the direction of caretakers who love the child. It is easy to see that each transition from function to function relies on the experience of love, pleasure, and trust. The paradigm for this reliance is set by the opening link in the transmission chain - the interaction between the mother and the infant that ensures the latter of mother's devotion and care. 
Certainly, it is hardly possible to verify how crucial the experience of motherly love is for the musical development of a child. Conducting such an experiment would be unethical. And under normal circumstances, the absence of a motherly figure in early childhood can be found, perhaps, only in a handful of "Mowgli cases" the records of which contain very scarce data on music capacities of such "Mowglis" [163]. The totality of what we know about ontogeny leads one to believe that without the mother's assurance and support through musicking, such as lulling, the entire development of a child in the domain of music would be impaired. This view is reinforced by what we know about the disastrous consequences of being deprived from linguistic stimuli during early childhood for further language acquisition, as in the so-called "feral" children.

This term refers to children who grow without human contact, either intentionally or through accidental circumstances. Feral children fail to develop many of the typical human abilities, particularly those that are needed for properly interacting with others and becoming integrated into society $[164,165]$. Perhaps the best known of feral children is Genie, a girl locked in a room until the age of 13. When she was discovered, she exhibited high levels of reactive aggression, avoided eye contact and touch, and was unable to speak [166]. Even after intensive training, the girl failed to develop a full-fledged language. This case was later used to support the hypothesis of the existence of a critical, biologically determined, period for language development in the child [167]. That said, most of feral children might have suffered from different underlying anti-social conditions, like intellectual disability (the case of Genie, according to [168]), autism spectrum disorder (ASD), or reactive attachment disorder (RAD), a condition with a genetic basis, like ASD, which is commonly diagnosed in children who have experienced extreme social adversity during early childhood $[169,170]$. Interestingly enough, these children can be argued to exhibit some sort of reversion of features associated with the "domestication syndrome" [171], which is a main concern to us in this paper (see section 6).

The entire foundation of music rests on the emotions that are opposite of aggression. Aggressive disposition towards a person automatically discards the possibility of musicking together in any form, whether active or passive. The state of aggression precludes cooperation necessary for transmission of music and breaks the transmission chain. And, judging from known indigenous cultures of personal music, strict self-communication is not sufficient to bring to life and shape new musical idioms. Even in the most isolated and sparsely populated areas, indigenous people musick interpersonally, usually between a parent and a child, and between marital partners. Generally, it seems that changing transmission types while switching between different functions is crucial for the crystallization of a musical idiom - especially, the transition from the tree to inverted tree format. The resulting canonization of the "beauty-of-average" version of a musical structure is instrumental for the emergence of musical semiosis.

All in all, experimental research on music transmission has not yet revealed any impact on compositionality [157], but found an increase in combinatoriality of rhythm [153]. From the musicological perspective, music follows rather few combinatorial rules - by a margin fewer than language - e.g., a leap in melody usually requires a step in the opposite direction [172], or, short rhythmic values require a longer value to terminate a rhythmic group [173]. However, such rules are usually quite loose: it is not difficult to find plenty of exceptions even in music cultures that possess fixed codes of music theory and formal schooling in music. The reason for this must be the specialization of music on prolonged exposure of a musical emotion. 
Unlike language that is designed to support quick real-time updating of referential information back-and-forth between interlocutors, music is designed to ensure that listeners experience a particular affective state. If changes of linguistic elements in a stream of speech most of the time directly add newer information for the listener, changes of musical elements in a stream of music most of the time do not change the meaning of music. Normally, musical meaning is changed by changes of musical themes, which occurs rather rarely. Majority of musical works outside of Western classical tradition feature one theme per work. If a non-Western music work is based on two or more themes, each theme requires a considerable exposure (about 2-3 minutes or longer). The most informatively dense forms of Western classical music, such as a sonata form, feature the shortest exposures of themes (sometimes as short as about half-a-minute), which still is by a magnitude longer than meaningful units of language - words.

The primary means of compositional semiotic arrangement for music is not the micro-level of musical elements but the macro-level of discrete sections of music form, dedicated to the exposition, variation, development, or recapitulation of a theme. For many forms of music, combinatorial organization does not really matter: i.e., rotating two meaningful elements does not alter the expression of a phrase that encompasses them. However, compositional organization of higher order structures is much more semantically susceptible. Thus, rotating prelude and fugue in a Baroque genre of "prelude and fugue" would drastically distort the rhetoric plan and the overall meaning of the entire cycle intended by the composer [174]. Such "fixed" cyclic music forms by no means are limited to Western classical music: e.g., there are Andalusian, Moroccan, Turkish, Persian, and Indian suite traditions [175].

The fundamental difference from language is that "syntactic" elements of music supply the musical theme with shades and tints of meaning rather than add little pieces of new meanings. Therefore, transmission of music structurally shapes only the thematic elements - those structures that readily become salient in conveying a specific musical emotion - leaving the rest of the musical elements up to chance. Often, a novice learner simply does not notice a supportive musical element, focusing his attention solely on the most salient thematic element. In such a case, the transmission chain for the secondary element becomes broken. That is why the repertory of music idioms is overall so vague and unequal. Thematically common idioms are relatively well structured (e.g., fanfare), to the extent of being verbally definable in a manner of a dictionary [123], whereas thematically uncommon idioms, whose role is primarily supportive (e.g., Alberti bass), have rather indistinct semantic values, so that it would take a long musicological essay to define them semantically. Another reason for semantic vagueness of music lies in its integrative nature: multiple aspects of expression each provide supportive idioms and the listener extracts their aggregate meaning. If an idiom or two is missed, the rest of the idioms suffice to convey semantic information. The consequence of this is that the competence of master-musicians widely exceeds the competence of average folks, especially in music cultures, where active production of music is reserved to only those who are considered musically gifted and/or musically schooled. Master-musicians usually implicitly acquire extensive glossaries of music idioms across all aspects of expression in music styles and genres they regularly practice.

Consequently, music transmission fundamentally differs from language transmission by not increasing the contrasts between musical elements to make their perception clearer and by simultaneous combination of multiple idioms, reserved for different aspects of expression. For example, a bossa nova theme can be recognized by the agile syncopated rhythmic figure placed against a smooth lazy melody, accompanied by the long non-repetitive progression of complex 
chromatic chords, whose harmonic resolution is postponed until the end of the progression [176]. Folk bossa nova musicians easily recognize and reproduce these features by ear.

One of the ramifications of the merging nature of musical "syntax" is that its transmission is strongly influenced by the number of participants and their auditory skills in recognizing specific acoustic attributes of sounds. The fewer participants, the less transparent signal mappings are, and the greater dependence of semiosis on the iconic encoding. From this angle, music transmission is similar to language transmission. However, if in language iconicity occupies little place, confined to such rather rare phenomena as onomatopoeia or ideophones, for musical communication iconicity constitutes the status quo. Multiple cross-modal (informally speaking, "synesthetic") correspondences between hearing music structures and associating their properties with properties of visible or tangible physical objects and patterns of their motion make perception of music quite intuitive, enabling music to represent objective reality [53].

Musical intonation is usually ascribed semantic values, based on similarity of its salient acoustic attribute to a salient feature of a semantic value (thus, descending melodic intonation receives a semantic value of relaxation, where actual reduction of intensity serves as an iconic feature). Musical rhythms are iconic in the same way. Iconicity makes implicit learning of music easier than implicit learning of language similarly to how onomatopoeic words are easier for a young child to learn than non-onomatopoeic. For this reason, as well as because of the high thematic redundancy in a music work, learnability of music tends to have less formative power over its transmission and is not always effective in counterbalancing expressivity. Active personal forms of music, cultivated in indigenous societies of Northeastern Eurasia amongst scarce populations, rely on the linear, circular, and inverted tree formats of transmission, which strongly regularizes the personal tune and conserves its salient (thematic) features. Active collective forms of music, cultivated in densely populated areas, such as in northern India, mostly engage the tree, inverted tree, and hybrid tree transmission, which promotes expressivity and innovation.

A very important note on music transmission is that the inherent connection of music to emotion and cross-modal referencing of certain cultural environments (e.g., social structures: mother-child in lullaby or battalion in march) and natural environmental features (landscape: water in chanties or mountain in climbing songs) make the task of acquiring command of music communication necessarily emic. The iconicity of music makes most indigenous musical traditions somewhat deictive - the iconic attributes must be observed by communicators in their original cultural and natural environment in order to be easily learnable and appropriately retrieved when needed. Thus, a song designed for walking on a plane surface features very different structural patterns than a song designed for walking up the mountain.

Similar correspondence seems to apply to the phonological bases of languages, whose ties to the geographic and cultural environment appear stronger in early stages of the evolution of languages. Recent research, based on extensive language databases, showed that some phonological features might have been influenced (and can be explained) by the physical environment in which they are spoken - e.g., the effect of vegetation on sound inventories, which finds parallels with vocal communication of many vertebrates [177]. Another example is the negative effect of dry climates on tone usage [178]. The global distribution of tonal languages that are concentrated in tropical and subtropical regions might be determined by the perturbations of phonation caused by desiccated ambient air. Interestingly, this effect is confirmed by historical linguistics. Eastern and Central-Western Bantu languages that moved to drier climates are known to have generally fewer 
tonal contrasts. Nonetheless, the tone systems of the languages within these sub-groups that reentered wet areas became more complex [179]. Pretty obviously, the effect of the physical environment on language change and language diversity can be direct, but more often, it occurs indirectly through the agency of ecological factors (e.g., shortages of food supply or spread of diseases) as well as demography (migrations and population contacts, or changes in the structure of social networks) [179]. It is, therefore, not surprising that the strongest environmental effects on the structure of languages are related to social factors, such as the looseness or tightness of social networks, the proportion of adult learners of the language, the degree of bilingualism, or the number of speakers [363].

Since linguistic phonemes come very close to timbre-classes of timbre-oriented music, it is reasonable to expect that geomusicological and geolinguistic traits share the same origin and contain similarities: e.g., Tuvan language features the unusual pharyngeal vowel and consonant harmonies, and pharyngealization constitutes the regional trait of a number of neighboring languages of the Baikal-Sayan area [180]. At the same time, Tuvan traditional music is characterized by a rich arsenal of deep-throat techniques of singing [181], also found amongst the neighboring ethnicities of the Altai-Sayan area [138].

Having our definition of music, its functions, and transmission formats in mind, we can proceed now towards critical examination of the latest takes on the evolution of music, especially the social bonding theory that has recently gained support from scholars of different fields of study.

\section{The evolution of music: an overview of the latest literature}

Over the time, after the "classic" introduction into this issue by Wallin et al. [182], many different theories about the origins of music have been posited. It is beyond the scope of our paper to evaluate all of them (but see the most recent updates [183-192]). Nonetheless, for a better understanding of our own hypothesis and its contribution to the field, we now provide a very succinct summary of the most recent developments.

Overall, theories about the origins of music fall into two basic groups: exaptationist accounts and adaptationist accounts. Exaptationist's accounts support the view that music emerged as a nonfunctional by-product of some pre-evolved ability/capacity, like language, audition, signaling vocalization, sound imitation, motor abilities, or even broad cognitive abilities like Theory of Mind. In a recent paper, Mehr and colleagues [9] provide several reasons that dismiss such exaptationist views and argue that music was selected because it provided humans some evolutionary advantage(s). Many other species are known to engage into music-like behaviors (like entrainment to a beat) and produce complex, song-like vocalizations that are socially learned and fulfill specific roles (mating, territorial defense). Mehr and colleagues consider human music the extension of such communication systems and set forth the following reasons to support their adaptationist view of music:

i) like language, music features complex design, where some aspects are universal while others are culture-specific; 
ii) newborns show an innate predisposition to distinguish music from other auditory stimuli, without the need of any extensive training;

iii) music perception relies on a specific neuronal substrate, the impairment of which causes distinctive music-specific damage;

iv) music is not a recent cultural invention, but an ancient capacity; and

v) all human cultures have developed musical behaviors that fulfill specific roles (rituals, ceremonies), whose form-function links remain pretty stable across cultures and societies.

These parallels between language and music are intriguing and have been examined in detail by several authors (see $[193,194]) .{ }^{23}$ Overall, it makes sense to argue for a human musicality (i.e., an innate predisposition to recognize, process, and create music, encompassing all the biological, perceptual, and cognitive aspects of music) that parallels our linguisticality (after Haspelmath [195]). Provided that the child is raised in a suitable environment, musicality results in the acquisition of the skills necessary for perception and production of music works that are deemed important within that child's cultural and social environment. Musicality relates to music pretty similarly to how linguisticality relates to languages: human linguisticality is expressed in several thousands of languages from which an infant selects those that are spoken around and acquires them in order to secure the needed care from caretakers.

According to some views (famously, Brown's musilanguage [2]), language and music share a common origin, although they later bifurcate in their evolutionary development. There are 6 possible ways in which music and language could have related to one another [196]:

i) autonomy of the music and language components within musilanguage and thereafter;

ii) inseparable origin in musilanguage, followed by divergence and autonomous development;

iii) inseparable origin and inseparable further development;

iv) full autonomy within musilanguage, followed by their convergence;

v) music emerging as language's offshoot (Spencerian view) or

vi) language emerging as music's offshoot (Darwinian view).

Of these theoretic possibilities, only 3 find support amongst modern scholars: ii) [2], v) [197], and vi) [198]. The second scenario seems to be gaining consensus, challenged primarily by the Darwinian view, while the Spencerian view is supported by fewer scholars.

Because of the shortcomings and limitations of exaptationist approaches to the evolution of music, diverse adaptationist accounts have been proposed instead. While most of these views argue for an extra-individual role for music, generally related to the establishment and consolidation of social bonds within human groups, some adaptationist views argue instead for an "internal" role, usually related to the management of emotions. For instance, Perlovsky supports the view that music helps us overcome what he calls "cognitive dissonances", i.e., unpleasant emotions resulting from our confrontation with the world's contradictions [186]. As he sees this, music helps to

23 Although it is beyond the scope of this paper to review in any depth the parallels between music and language, we do occasionally draw specific parallels between them for 3 reasons. First, because they both might have common origins in musilanguage. Second, because evolutionary linguistics has explored in detail the language origins, we might build on some of its findings and even methodologies. This will be particularly true for section 7 (our model). The effects of the environment on structural aspects of language (as noted above) constitute yet another area where the findings by linguistics can be useful for musicology. 
achieve some sort of comforting synthesis of otherwise antithetic impulses triggered by our conscious interaction with the world. Interestingly, Perlovsky also argues for a common root of music and language in animal vocalizations, with some vocalizations detaching from communicating emotions and evolving into language, and with others preserving primordial emotional connections and evolving into music.

As noted, most adaptationist hypotheses of music evolution argue for an "external" role of music. According to some views, music evolved to create and maintain social bonds as human groups grew larger, and primate-like social bonding behaviors (like grooming) turned to be inefficient (see Dunbar, [199]). This is the essence of the "social bonding" hypothesis mentioned above, which argues for a positive effect of music on diverse hormonal and neurobiological mediators of prosociality: the stress-response system that counteracts psychological stresses caused by social living which threaten the cohesion of the group [199], the endorphin producing system that favors a positive attitude towards others [200], and the dopaminergic reward system [10]. Significantly, rare cases of musical anhedonia result from altered connectivity between auditory and reward systems [48]. At some point, the "internal" and the "external" accounts of the rewarding effects of music can conflate. Thus, according to Harvey [192,201], music can promote group coherence, as well as personal well-being.

According to other views, the motivation that underlies music evolution was not the rewarding effect of music on the biology of the performers (with the aim of favoring the desired social behavior), but the implementation of a way to convey credible information to others. For some authors, such credible information concerns the physical and even cognitive condition of the sender, so that music is hypothesized to have resulted from sexual selection, evolving to signal mate quality (e.g., [202,203], among others; see [9] for some criticism, particularly, the weak link between sexual dimorphism and musical ability/production). For other authors (most notably, [9]), music evolved to provide increasingly sophisticated credible signals that are needed to cope with progressively complex social conflicts of interest, like those resulting from multi-level social organization or higher levels of (allo)parental investment. Contrary to the view of music as a social bonding device, under this view music just makes social cohesion more perceptible rather than directly causing social cohesion that has been obtained by different (non-musical) means - music merely signals already existing cohesion.

This important conclusion is in agreement with our summary of operational functions, their evolution and transmission of music, presented above. Indeed, the establishment of a bond precedes the onset of musical communication between mother and infant, and active musicking on part of an infant starts at least a year after passive exposure to motherese. There is no reason to think that cultural evolution of music did not follow the same path. Had music possessed inherent power to cause bonding, it would not have been used on the battlefield to intimidate the enemy. To the contrary, Titus Livius in his "History of Rome" reported that in the 4th century BCE Gallic troops went into a fight singing dissonant battle chants. Sassanian martial music used kettledrums and battle cries to undermine the morale of the enemy [204].

However, despite the rightful critique of the exaggerated exaptationist accounts of the bonding function of music, Mehr and colleagues [9] side with the exaptationists in their statement that "music is clearly rooted in sociality". Compare it with the conclusion of Savage and colleagues: "dancing to an isochronous beat with a metrical hierarchy, singing learned melodies based on discrete scales in harmony, using predictable, repetitive musical structures, and using musical performances as cues for social identity - are widespread throughout the world's musical systems. 
These features have clear functions for group performance, but little or no function in solo performance..." [10]. This generalization is simply erroneous. Many ethnicities whose indigenous music traditions are strictly monophonic utilize metric organization, discrete pitches, formulaic design and use music to identify an individual [12].

The huge geographic area north of Japan, Mongolia, and Afghanistan, stretching from Chukotka and Kamchatka to Finland and Scandinavia, and then Greenland, Canada and Alaska are populated by people who do not cultivate choral singing at all. Music of the indigenous population of the entire continents of Australia, North America, Andean area in South America, East Africa, upland minorities of the Golden Triangle of Burma, Laos, and Thailand is predominantly monophonic (see $[205,206])$. The overall geographic prevalence of monophonic music is obvious from the map from the International Research Center for Traditional Polyphony ${ }^{24}$ (Fig.4).

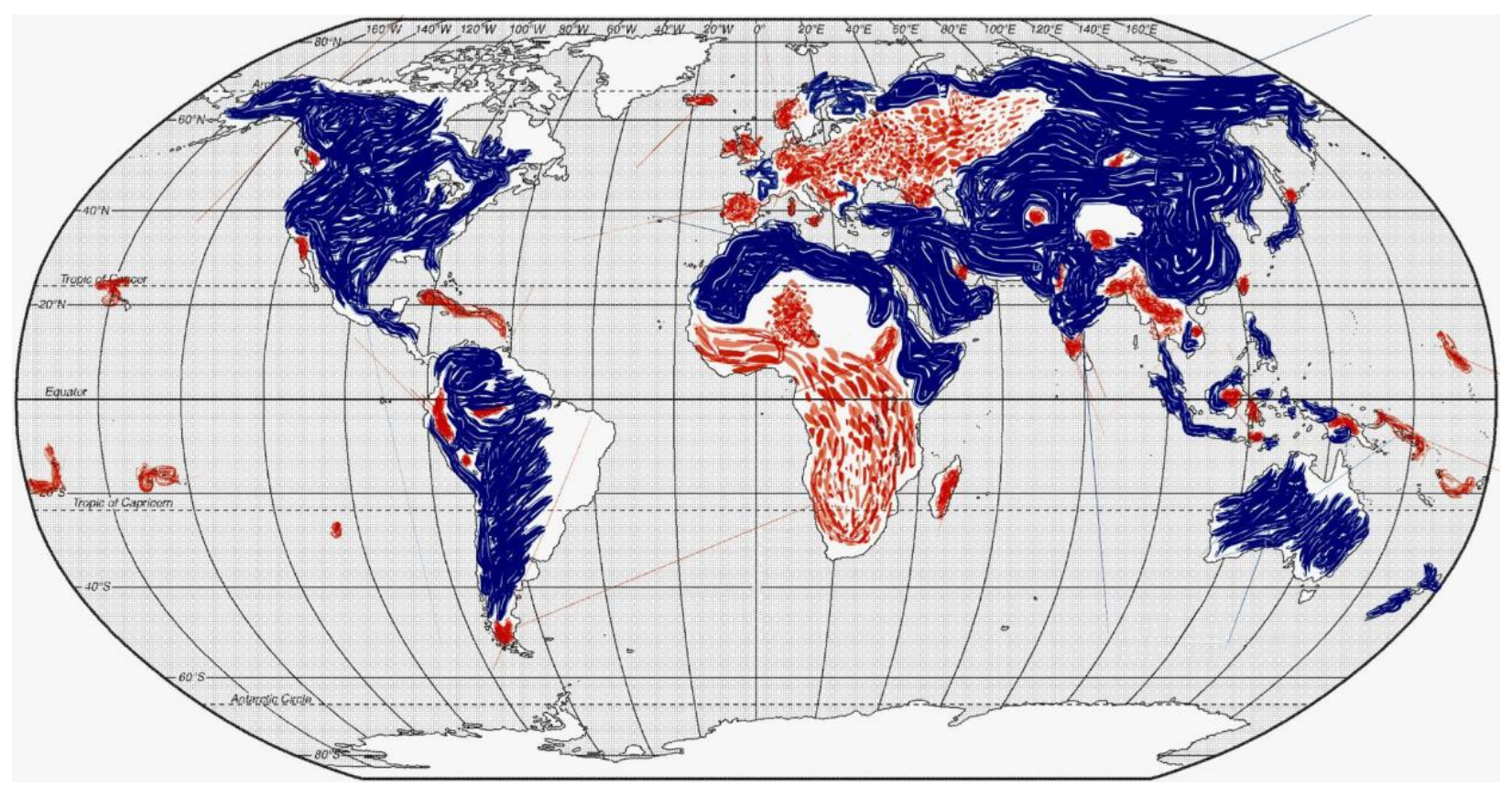

Figure-4. Global distribution of multipart and monophonic music. Blue color marks the areas inhabited by the ethnicities that have monophonic indigenous music traditions and orange color - those with multipart indigenous traditions. Courtesy of Josef Jordania. Used by permission.

In reality, monophony prevails over multipart music even more than the map shows, because many multipart cultures contain monophonic music genres, whereas the majority of monophonic cultures

\footnotetext{
24 The term "polyphony" is often used indiscriminately. According to the Oxford Dictionary, polyphony is "a style of simultaneously combining a number of parts, each forming an individual melody and harmonizing with each other". The criterion of multi-melodic arrangement has been emphasized as a key distinction between 4 major typologies of textural arrangement - polyphony, homophony, heterophony, and monophony in the corpus of literature on music theory [207]. Unfortunately, the current consensus of ethnomusicologists has blurred this criterion in the ethnomusicological definition of polyphony: "a mode of expression based on simultaneous combination of separate parts, perceived and produced intentionally in their mutual differentiation, in a given formal order" [208]. Instead of clearly stating that polyphony is characterized by the simultaneous exposure of multiple melodies and naming principal structural cues for identifying a melody, this definition puts forward a very vague structural criterion of "intentional differentiation" in a "formal order". As a result, in modern ethnomusicological literature, especially coming from Western countries, homophonic textures are often mistaken for polyphonic. This misnomer probably stems from the increasing trend of the lack of training in the structural analysis of music in the curriculum of many Western universities [209]. For the context of our discussion, the musicologically accurate term to refer to the opposite of monophonic musical arrangement is "multipart music" (which encompasses both polyphony and homophony).
} 
do not contain multipart genres. If music indeed is rooted in sociality, then why do the population of such huge areas of land not practice forms of music that are impossible to perform other than by the collective effort of a group? Jordania argues that initially all world's music was multipart but thereafter suffered decline and was converted to monophony [205,206]. However, Jordania's explanations do not answer why would masses of people all over the world lose the need to incentivize social bonding, if the latter is the primary reason for music's existence? Quite the opposite should be expected in light of the consistent growth of the world's population, starting from the Neolithic, and the increasing technological importance of cooperation. In fact, some highly technologically developed civilizations with a large population and oldest urban cultural tradition completely miss the tradition of collective singing - not even of a monophonic melody! Thus, when Bartok tried to record samples of choral music from Anatolia in the 1920s, the locals simply could not understand what he was asking for, because everyone sang only solo [210]. ${ }^{25}$

The pattern of acquisition of music by children delivers another strong argument in favor of the precedence of monophony over polyphony. No infant has ever been reported to spontaneously sing polyphonically in the counterpoint to another singer - not even sing in tune a monophonic melody along with someone else. All reported accounts of spontaneous music-making before the age of 23 are either monologic, dialogic (i.e., responsorial), or masking some musical background (an infant becomes excited by musical stimuli and starts cooing without any coordination in pitch or rhythm to those stimuli) [29]. It takes children about 4-5 years to learn to sing together with someone else in a coordinated manner - and this is only in societies where choral singing is commonplace. Putting this differently, a solo tonal organization has to crystalize first in order for a polyphonic tonal organization to evolve in an indigenous music culture. Jordania (as well as a number of other scholars) puts forward the Pygmy polyphony of Central Africa as a model of the primordial form of music that supposedly was carried out of Africa to the rest of the world [205]. However, the Pygmy children follow the same developmental path of first mastering monophony before exploring duetic and polyphonic singing and are nurtured by the strictly monophonic motherese [212].

Overall, the available evidence suggests that genuine chorusing in a group as a single entity ("choir") to share emotional content and/or to strengthen social bonds is a higher order phylogenetic and ontogenetic construct. In truth, without a well-established monophonic tradition, no formation of music modes, scales, divisions of rhythm, and metric typology is possible - not to speak of passing the musical skills to handle such schemes over generations in quantities large enough to secure their survival as a tradition. Important for our concerns here, learning musical intervals and singing in tune demand the format of a face-to-face responsorial, where the teacher shows the model, the learner tries to imitate, and the teacher corrects the committed faults. Accordingly, polyphony demands long face-to-face interactions to emerge.

It seems that proponents of the bonding theory drastically overestimate the spread of multipart music and the place of bonding function in the timeline of the evolution of music. Indeed, social bonding becomes more and more important as the density of population keeps increasing. This is clearly manifested in the global tendency of frequency-oriented music traditions to supplant the timbre-oriented ones in those ethnicities that adopt the settled lifestyle and live in large groups

\footnotetext{
${ }^{25}$ As Bartok's interpreter, Saygun explains: "It is important to observe that the Turks do not enjoy singing together as a group. In fact, both amongst villagers in Anatolia and students at city-schools, solo singing is more dominant than group-singing. One of them sings a "Turku" [a popular folk song] and the others listen. Even occasionally when others join in, they often sing out of tune... This does not mean to say that the Turk does not have an ear for music, it simply shows that they have not been trained to sing as a group" [211].
} 
(timbral music affords only close-range use that supports no more than a handful of participants). However, the sustained population growth has started no earlier than from the Holocene - just a "tail" in the timeline of human evolution (about 3\% of the 300,000-years-long course of human evolution). Social bonding obtained the pronounced formative influence on tonal organization of music and its semiosis only at later stages in its evolution - after the emergence of city-states and palace/temple professional music (ca 30th century BCE).

Equally overstretched appears the idea of direct tying the population size to the passive use of music: e.g., "once group size increases substantially, it may not be feasible for all individuals to participate actively in a coordinated manner, but music can facilitate bonding via passive (including digital) participation" [10]. Group size per se does not prevent partaking in the collective music performance - even for those who cultivate monophonic music. Thus, Yakut musical tradition, primarily monophonic, solitary and restrained to musicking of no more than 23 close relatives, features a single massive annual festival, celebrated by the collective ritual rounddance osoukhai [213]. In 2012, in Us Khatyn, it broke the world Guinness record (registered by the Guinness judge Jack Brockbank): over 15,000 people simultaneously participated in dancing and singing in 36 gigantic circles.

As we discuss in more detail in the Sections 6-7, the key factor in genesis of prehistoric music (and language) seems to be the population density and its interplay with the environmental conditions: harsh enough to promote prosocial behavior and to trigger the acceleration in the development of new sustenance technologies, yet not too harsh to bottleneck the transmission of knowledge over generations within the territory large enough to secure the survival of novel traditions. Those few ethnomusicologists who still conduct comparative study ${ }^{26}$ consider 2 alternative scenarios of the rise of music: African polyphony (e.g., Jordania, Arom, Grauer) and Siberian monophony (Alekseyev, Sheikin, Zemtsovsky). "Hot" African model, characterized by relatively high population density prior to the Last Glaciation, suits the "polyphonic" scenario of very gradual evolution of collective musicking, exported from Africa. "Cold" Siberian model, characterized by demographic scarcity and the institute of personal song, suits the "monophonic" scenario of emergence of music through the "art revolution" during the last Glacial.

Of particular promise are two emerging approaches to the investigation of the origin of music. The first of them grounds human musicality on broad aspects of human embodied cognition [188] and on abilities and capacities that are shared with other animals, like vocal learning and beat perception [191]. Such evolutionary continuity and generality of the biological underpinnings of human musicality is in line with recent accounts of human linguisticality, which find continuity with the cognitive and communicative abilities of other species (see [216-218] for recent views). The self-domestication hypothesis of human evolution [1], which is central to our hypothesis, also falls within this type, since it acknowledges a great degree of continuity between human evolution and the evolution of other species - e.g., bonobos are claimed to have also gone through a selfdomestication process, like humans [219].

\footnotetext{
${ }^{26}$ Unfortunately, the view that ethnomusicology should altogether avoid cross-cultural comparisons in order not to hold one culture in any way higher-ranked than another and the resulting denial of global evolutionary development of human music have become a major obstacle for bringing the comparative study of music on par with comparative linguistics [209]. Notably, Bruno Nettl, often cited in the literature on psychomusicology and evolution of music, in just a decade has reverted his views expressed in his chapter in the now classic "The Origins of Music" [214] and pronounced the legacy of Wallaschek, Stumpf, and Hornbostel outdated [215], thereby denouncing his own earlier call for searching for musical universals and establishing the landmarks in cultural evolution of human music.
} 
The second approach is based on the idea of a coevolution between musicality and music, where both were mutually enhancing and shaping each other. Thus, van der Schyff and Schiavio [220] argue in favor of a "biocultural" approach to the evolution of music, which holds the biological and cultural dimensions as aspects of the same evolving system. Likewise, Savage and colleagues [10] argue in favor of the coevolution of music and musicality via iterative niche construction, driven by their positive effect on human social bonding. This approach parallels that of modern evolutionary linguistics. Languages have been shown to impact our cognitive architecture in order to support quicker and more efficient mental processing [221], to the extent that gene-culture coevolution is expected to be a relevant component of any narrative of language evolution $[222,223]$. Along the same lines, cultural niche construction has been hypothesized to have contributed to some of the features exhibited by modern languages, and, more generally, to our linguisticality [224].

It seems that our cognitive architecture imposes specific restrictions on the structural features of languages as well as music. Some sort of gene-culture coevolution must have accompanied the emergence of complex languages and music systems under the effects of reduced reactive aggression and increased prosociality via its effects on cultural niche. Changes in the management of human aggression could account for most aspects of the cultural niche that enables the growing sophistication of language structure via a cultural mechanism [225]. Aggression management mechanisms, growing language complexity, and changes in ways of using languages all might be inter-connected by a feedback loop [226]. Similar feedback could connect aggression management with progressive sophistication of music syntax and functions that music typically executes.

In our model (Section-7), we will adhere to the possibility that music and language evolved independently from a common origin in musilanguage, as also argued by Brown in his updated model of musilanguage [227]. Hence, their common roots urge for a re-aligning of musicological and linguistic methodologies [15]. In this paper, we shall follow this direction, relying on the human self-domestication hypothesis of language evolution [1]. We expect this approach, when properly qualified and improved (see Section-6), to help us reconcile hypotheses about music evolution that have been presented by their authors as irreconcilable. But before doing that, we will examine in detail the nature, origins, and social functions of personal song. One reason is that, in our view, it can be construed as a sort of bridge between our pre-adaptations for music (particularly, animal calls) and modern forms of music (particularly, the type of music that emerged in the Holocene). A second reason is that the factors that contributed to the genesis of personal song provide an insight into how environmental influence (natural and cultural) can transform music. Changes in the management of aggression through the course of human evolution that we discuss in Section-6 very much depend on our analysis of the genesis and evolution of the institute of personal song.

\section{5. "Personal song": the missing link between the animal calls and human music}

The ethnomusicological data confirms that credible signaling hypothesis [9] is generally right in claiming that music emerged as the "credible signal". In most surviving indigenous folk music traditions, the performer still, as a rule, does not "lie" through musicking but conveys what is in 
his heart and mind. Whether he performs pre-existing songs, created by other musicians, epic tales, where he must speak for various protagonists, or shamanic kamlaniye, which requires impersonations of spirits and souls of other people, he and his listeners believe that he temporarily becomes "possessed" by some other power that communicates through his mouth [228]. Even in reproductions of popular "cover-songs," a folk singer typically conceives the lyrics as "direct" rather than "indirect" or "scripted" speech [229]. Often, music directly charges the performer, who was not disposed to the musical expression of a pre-existing song prior to its performance, with the musical emotion of a song, which changes that performer's emotional state to match the musical emotion. And listeners take a rendition of a music work at its face value: things that the performer conveys stand as "true" on his part at the moment of performance.

Deliberate "putting on an act" as a method of performance practice can exist only in music that segregates the performer, the composer, and the listener - which is solely Western classical music and its offsprings (musicals, commercial popular and folk music, etc.). And even in this tradition, insincerity and "emotional faking" on part of a performer is regarded as a fault that characterizes the prevalence of mercantile concerns over aesthetic ones, and is associated with the downside influence of the entertainment industry [230]. A good classical performer is expected to treat a musical composition as a "method-actor", living through the emotional experience of a protagonist he impersonates [231].

Altogether, music has remained a categorically "honest signal" for most of its evolutionary development and only over a few last centuries has acquired the capacity to "pretend" [232] which is hardly enough to pronounce music "dishonest" by design. ${ }^{27}$

The premise of "honesty" is a crucial point of difference from linguistic communication, aimed to convey propositional meanings, where "white lies," flattery, and "political correctness" occupy an important place. The principal reason for this seems to be the non-synchronous nature of verbal communication - speakers take turns. Collective utterances by a group of people, representative of their true state, can bear no continuously updated information, mutual for each of the participants, and usually express an affective state or attitude, which stays unchanged for all of them. Strict soliloquy delivery, on the other hand, facilitates the manipulation of a listener, including intentional misleading. Collective experience of the same affective state makes deliberate lying and pretense difficult. In a small group setting, which is the most common form of collective performance globally, a singer who does not share the same musical emotion with his partners appears insincere or inexpressive to his partners and listeners. Any ethnomusicologist with experience of field research would testify that when an invited performer does not feel the same as his partners in relation to the expression of a requested song, that performer usually refuses to participate [65].

It is important to underline that the antithesis of fundamentally "honest" music and often "dishonest" language is limited to the later evolutionary stages in their development, when both were cultivated amongst large complex populations (e.g., tribal confederations and early states) after the beginning of the Holocene and before the 18th century, when the tradition of musical acting was forged in the performance practice of Western instrumental classical music (within the doctrine of Affektenlehre). During the earliest stages of music and language evolution, levels of

27 As far as we know, the only scholar of the evolution of music who explicitly concluded that music is based on deliberate deception of the audience by a performer is Trehub [233]. However, she has never responded to the criticism of her position by Juslin and Västfjall [234]. 
aggression were still high, so that most uses of language must have been expressive by nature and reflected the genuine emotional state of a speaker - very much like music. In other words, we could confidently expect that the earliest forms of language were also an "honest" signal, and that white lies and the likes only emerged later, as language started specializing in transmission of objective meanings. The initial "honesty" of language can be imagined based on the ethnographic reports of the first travelers in the north-eastern Asia - e.g., the verbal communication of the Yukaghirs [235].

The roots for the default "honesty" of musical communication in collective settings are best understood by looking into the tradition of personal musicking. We have already mentioned a peculiar cross-cultural phenomenon of personal song that constitutes a unique institute of social and geographic identification of an individual in numerous indigenous cultures in the Northern hemisphere [12]. In many traditional societies there, every member is assigned a personal song (usually soon after birth by the parents, to be replaced by an original composition upon puberty). By its melodic and rhythmic structures, the personal tune reflects the characteristic personal traits, general temperament, and age of its owner and by its similarity to personal tunes of other people it indicates song-owner's ethnicity, kin, and place of origin to those familiar with these conventions. Throughout one's life, a personal song is used as a musical "clone" of its owner - an aged person changes a personal tune to reflect lack of energy, one sings a song of a dear relative when departed (which is believed to communicate to the soul of that relative), singing one's song by an unrelated person is regarded as inflicting harm on the owner, shamans heal people by evoking their personal songs (using a personal tune like a Voodoo doll). Song owners often modify their personal tune to make it match their current emotional state or activity - as though constantly reflecting musically on their existence. Much of the day of an indigenous person is dedicated to such singing. Sheikin calls it Cartesian: "I sing, therefore I am" [138].

Just like the use of fake passports is punished by law, indigenous societies protect the honest use of personal song. The latter is considered the valuable property of its owner that can be given away as a gift, exchanged for some goods, or inherited. Unauthorized singing of another person's song is punishable by the owner of the song and his relatives. The entire society is likely to ostracize the offender, causing dire consequences of living in harsh conditions while relying only on oneself. Even more scary to the natives appears the prospect of punishment by the spirits that patronize the owner's clan.

The institute of "personal song" seems to present an evolutionary extension of the functionality of animal calls, specifically, credible signaling of social animals. Such signals are used for identification in mate choice, individual recognition in interactions among conspecifics (e.g., territoriality, dominance, and cooperation), and for displaying formidability, health, or behavioral type of the signaler. Many structural aspects of such calls "are shared by all species members, each individual employing some fraction of the universal lexicon" while "providing ample acoustic vehicles for local dialects and individual differences" [236]. Some birds even create novel song types by recombining "syllables" of their initial songs. The specific structure of a birdsong informs the conspecific birds of the singer's identity, membership in a local group, its belonging to a certain species, and genetic quality [237]. Incidentally, similar signaling systems, including the rearrangement of syllables in order to identify an individual animal, have been found in primates [238].

This is remarkably similar to the human personal song that reveals a singer's ethnicity, place of origin of his parents, kin, and age by means of re-arrangement and specific modifications of 
parental songs [239]. Likewise, the creation of novel song types by recombining elements of previous songs, encountered amongst some animal species, stands in line with the composition method by which Siberian natives generate personal songs for their newborns. Moreover, the division of the animal species-specific system of signaling into local "dialects" and individual styles across the animal kingdom closely resembles the geographic and ethnic distribution of personal songs in Siberia and the Russian Far East. Each ethnos possesses its own melodic, rhythmo-metric and timbral markers, and different geographic communities of that ethnos (e.g., tundra vs. forest Nenets) employ "local dialects" of a tribe-specific style of a personal song. Interestingly, many of the indigenous people do not consider personal song as "music", regarding it as a spiritual phenomenon that has to do with one's soul. This special status supports the possibility that personal song originally evolved directly from musilanguage, prior to formation of music as we know it.

Finally, just like animal communication, the entire institution of personal song is based on the issue of territoriality and mating. The emergence of personal song is attributed by the experts to the need to mark the location of one's residency and minimize incest marriages through securing the possibility to take a wife from the neighboring tribes. This cross-cultural Siberian custom (present amongst almost all its indigenous peoples) tends to make each ethnos friendly to its neighbors - bringing to mind the "dear enemy effect": i.e., a tendency of the neighboring territorial animals to become less aggressive to one another once the territorial borders are well established [240]. The "dear enemy effect" seems to be determined not by the need to defend one's territory because of feeding, but it has to do with breeding [241]. Overall, at least in some sense, the institute of personal song represents the intermediate stage in the transition from animal communication to human music.

Both the hypothesis of music as a credible signal and, particularly, the hypothesis of music as a social bonding device, support the view that music strengthened links between individuals via the exchange of information or/and the collective experience of living through the same musically supported event. Nonetheless, both hypotheses assume that human demography in the past allowed such collective meetings and particularly, that human beings were prone to interact with others. In truth, during most of the Middle and Upper Paleolithic population was very sparse, mostly due to the deterioration of the climate conditions. For instance, demographic estimates for the huntergatherer populations during the Aurignacian period ( 42-33 kya) point to a mean of 1,500 persons for Western and Central Europe combined, extending into the early Gravettian period [242]. There is evidence that harsh climate changes affected not only Europe and North America but Northern Africa and Sahel as well [243]. Accordingly, the population density of Magdalenian Europe during the Last Glacial - 0.036 person/km2 [244] - was close to the population of Northern Siberia prior to its colonization by Russia in the 17-18th centuries: 0.065 person/km2 [245]. Hence, in our opinion, showing off the qualities of one ethnos to impress another ethnos, not to say to promote social bonding, was barely possible on a systematic basis due to the low population density. Even if such music was originally created in warmer and milder climatic conditions in the vicinity of Horn of Africa and exported to the Western Asia and Eastern Europe, it must have been radically transformed by the millennia-long episodes of cooling, so that by the time of the beginning of global population growth in the early Holocene, the entirely new tradition of musicking was in place.

Much more likely scenario is that the primary motivation for the use of music during the Middle and Upper Paleolithic was not inter-tribal competition, as many scholars suggest, but 5 principal 
goals of personal and private forms of music as practiced by the modern ethnicities of the Extreme North (see [12] for further details):

1) to mark one's social identity, territory, and temper through the personal song;

2) to support the ancestor cult, central to the kin-based ideology of indigenous populations; ${ }^{28}$

3 ) to reinforce self-awareness and sanity under stressful conditions (extensive period of solitude, long solitary travel in hostile weather conditions, during sheltering, illness, etc.);

4) to regulate one's interactions with the physical and cultural environment (rituals of hunting, marrying, burying, celebrating important events, etc.) for harmonious coexistence;

5) to win a favorable attitude of spirits/deities of local landmarks, crucial for sustenance.

The best idea of prehistoric human music seems to be the music culture of Samoyedic peoples in the Taimyr region: Nganasan, Enets, Selkup, and Nenets. The archeological evidence demonstrates that art forms of many modern indigenous ethnicities of Northeastern Eurasia strongly resemble the Mesolithic and especially Neolithic local artifacts, indicating an uninterrupted cultural tradition [138,247-252]. The ethnographic estimate of first arrival of proto-Samoyedic people in Taimyr is by the Mesolithic period, around 7 kya [253], and the earliest discovered settlement there is dated by no earlier than 8 kya [254]. However, a recent find of a mammoth kill site, with human-inflicted injuries, at Sopochinskaya Karga, east of the Yenisei Bay (c. $72^{\circ} \mathrm{N}$ ) was dated 47 kya [255]. This date agrees with the very reliable date established for the Ust'-Ishim man, found up the flow of the Ob' River [256]. In fact, the Ust'-Ishim man is the world's oldest remains of Homo sapiens that contain the complete genome. The consensus of geneticists places the Ust'-Ishim man in the first wave of humans that migrated out of Africa eastward, nearly 10,000 years after the incidents of human interbreeding with the local Neanderthal population (ibid.). Modern indigenous people of Siberia share $38 \%$ of their ancestry with the Ust'-Ishim man, and Western Siberians trace $57 \%$ of their ancestry to ancient North Eurasians, as represented by the 24,000-year-old Siberian Mal'ta boy [257]. The analyses of 34 newly recovered ancient genomes dated 31-0.6 kya show that the first inhabitants of northeastern Siberia were related to early West Eurasian hunter-gatherers and mixed with the newcomers from East Asia, which gave rise to Ancient Paleo-Siberians, closely related to far-Northeastern Siberians and Native Americans [258]. Siberia, the northern Far East and North America constitute exactly the heart of the surviving circulation of personal songs.

Although Ust'-Ishim is 1,500 km away from Sopochinskaya Karga, its inhabitants could have conveniently traveled North by the Ob' River, whose gulf is only $300 \mathrm{~km}$ away from the Yenisei River. The area between both rivers is populated by the Nenets and Enets who probably inherited the culture of Paleo-Siberians [259]. The archeological data suggests that the entire coastline stretch from Taimyr to Aldan $(2,400 \mathrm{~km})$ was occupied by a single gigantic Mesolithic culture, the homogeneity of which increased even further towards the later Neolithic [253]. This Neolithic culture is called proto-Samoyedic in Russian literature and is dated 6-1 kya, based on the data of paleolinguistics, paleobotany and lexicology [260]. Its breaking into currently existing Samoyedic cultures is dated by the middle of the 1 st millennium AD. Therefore, it is plausible to regard the institute of personal song as the remnant of a prehistoric music system that was spread across

${ }^{28}$ Indigenous Siberian understanding of kin within the belief system of the ancestor cult finds close parallel in the morphology of personal song. Perhaps, this correspondence is most illuminated in the concept of seok ('kin', 'bones', and 'cemetery') in Altaian languages - understood as "the quintessence of live matter, capable of securing future births" [246]. A trans-Siberian convention regards "bone" as a substrate that connects the deceased ancestors with their living posterity - so that all kin-members share the same bone ingredients. Within this framework, an individual is characterized by the re-configuration of the parental bone ingredients. This is essentially similar to one's personal song constructed from the characteristic elements of parental personal songs [239]. This parallel plays into the legitimacy of applying genetic models to the transmission of musical structures (addressed later in this chapter). 
Eurasia during the Last Glaciation, when the tundra belt covered most of Eurasia, expanding southward to $57^{\circ} \mathrm{N}$ (the latitude of Latvia), where it turned to steppe [261]. Flat landscape stretched from Western Europe to Beringia, enabling migration of animals and human hunters, chasing their prey all the way to North America [262]. It is through this migration personal song (aka "private song" in the US ethnographic literature) must have reached as far south of the USA as Sioux settlements in South Dakota, Iowa, and Illinois [263].

The most fascinating aspect of personal song as practiced by the Nenets is that the comparative analysis of the morphology of personal songs enables a competent listener to identify a Samoyed's genealogical tree [239]. One of the main specialists on tonal organization of Nenets music, Niemi, concludes that the principal reason, why the traditions of so many Siberian ethnicities do not contain collective singing and often completely lack musical instruments capable of generating multiple pitch classes, is the primacy of the identification functionality of personal song (ibid.). The latter imposes the need to recognize in real time the details in timbral, melodic, and rhythmic organization of a personal tune, discouraging people from simultaneous combination of musical tones, which obscures discrimination of structural properties of music, especially timbral.

Here we wish to highlight the timbre-oriented nature of "personal music". Multiple evidence supports the view that the timbre-oriented music preceded the pitch-oriented music that is commonly found in modern times in many places, particularly, in Western Europe. We have already touched upon the ontogenetic argument that all normal human infants start their acquisition of vocal skills from timbre-oriented vocalizations and gradually develop the conservation skills of converting timbral changes into pitch changes in processing melodic contours [29].

Another argument is that the tradition of personal music in Northern Eurasia and North America is amazingly conservative on a personal level - it has hardly changed at all in communities that maintain the traditional lifestyle throughout the past 200 years ${ }^{29}[136,138,264-266]$. Therefore, seeing Northern traditional timbral music as a "defective" emulation of "more accomplished" pitch-based music, borrowed from Southern neighbors, sounds highly unlikely. Why have millennia of emulation not resulted in acquisition of accurate discrimination of pitch on par with the neighboring pitch-based cultures? The likely answer is that timbre-oriented traditions of musicking rely on the modes of perception and processing of sound that differ from those of pitchoriented music.

Yet another argument is that there is no evidence of pitch-based musical traditions that were replaced by timbre-based traditions, whereas there are plentiful examples of the reverse replacement of an older timbre-based tradition with a newer pitch-based tradition, adopted from the politically domineering neighbors along with a new lifestyle and new sustenance methods (in Siberia and the Far East such influence usually comes from Russia and China). The reason for such one-sidedness seems to lay in psychoacoustics: it is easy to extract pitch from the audio spectrum by focusing on the periodic vibrations and finding the fundamental frequency for the identified harmonic content of the auditioned spectrum. And it is difficult to turn attention away from the harmonic content, once it has been discovered, and interpret a harmonious musical tone

\footnotetext{
29 This is aside from the natural decline of indigenous traditions due to migration of indigenous population away from native territories, settlement in towns and cities, and cross-marriages that led to substantial creolization of indigenous minorities. The currently observed pattern of weakening and disappearance of personal song in the regions of Middle Asia, South Siberia, and South Far East, geographically more accessible and open to trade contacts, migrations, and conquests [138], suggests that the original "Ecumene" of personal song during the prehistoric times spread substantially farther to south and west than Fig.5 shows.
} 
purely on timbral basis. The validity of this limitation is evident in the entire line of development of Western classical music tradition. It started with such forms of music where timbre carried a formative role - e.g., the so-called "florid organum" of the Notre-Dame school of the 12th century, where the upper part was defined as pleasant, light, and lucid, whereas the lower part as stern, firm, and dark [267]. And by the 18th century, classical music ended with disqualification of timbre to the secondary role of mere embellishment of pitch [268]. The latter is most evident in the widespread practice of transcribing popular pieces of music for all imaginable instrumental and/or vocal settings.

The "self" of a personal song is organically confined to the timbral aspect of expression via its implicit connection to the unique timbral characteristics of one's voice that allow friends and relatives to recognize the possessor of that voice upon hearing its sound recording. Personal song most certainly was brought to life by the function of recognizing a person by the sound of his voice - to address the challenge of identifying specific individuals in an ever-growing population. The need for unique auditory markers must have promoted the exploration of multiple aspects of expression, the most salient of which are pitch contour, rhythm, and timbre. The ongoing transmission of their characteristic patterns amongst the community members probably shaped the earliest forms of music communication. Ancestor worship, totemic, and animistic beliefs, so common in societies of hunter/gatherers, have posed the need to not only create a unique auditory pattern to mark every newborn member, but also to keep the auditory markers for the deceased members and to generate similar markers for ancestral figures of each kin and important patronizing deities. Siberian indigenous people define their place in the world by mapping themselves in their kin lineage, tracking it all the way to a totemic ancestor, and use the system of auditory indexation for orientation within their genealogical line [246]. That is why they know by heart many generations of their ancestors, well above the 3 generation standard of Westerners, and have amazingly sharp memory for personal songs, even those that they heard occasionally decades ago [269].

Intuitive morpho-syntactic analysis of personal songs of people and deities, whose relations are also modeled after human kins, constitutes the foundation of the system of auditory indexation that gives meaning to lives of indigenous northern Siberians.

The idea that the comparative morphological analysis can define the "life-history of a melody" and its geographic distribution, thereby helping reconstruct the long past of undocumented folk traditions was proposed in 1957 by Wiora [270]. The methodology for it was successfully developed and implemented by Zemtsovsky [271] and, independently, by Sipos [272]. Grauer noted that transmission of a song parallels the transmission of the human genome, and the task of establishing the evolutionary pathways of music can be achieved by tracking the distribution of "musical genomes" [137]. Jordania considered musical mode "the DNA of musical cultures" and used statistical distribution of modes of contrasting intervallic typologies to infer the global patterns in cultural evolution of music [206]. Savage brought the idea of genomic approach to music to a new height of science-based quantification by adjusting the approach of Dawkins' memetics (modeled on molecular genetics) to track variants in cultural transmission of melodies of popular and traditional folk tunes [273]. Finally, Jan elaborated Savage's technique to identify what he called "musemes" - i.e., perceptual-cognitive elements of music, revealed by motivic structure of songs - seeking to establish the parent-child relations between diachronic variants of the same song [274]. All of these "genealogic" developments have been criticized by modern Western ethnomusicologists for their alleged Eurocentric bias, scholastic estrangement from real- 
world practice of musicking, and wrongness of drawing parallels between genetic and cultural heritage. Such critics themselves are proved wrong by the naturally evolved ancient institute of personal song, where the family of personal tunes in the most direct sense represents the genealogical tree of a given kin by virtue of reusing structural elements of parental songs (morphology) and methods of tonal organization, characteristic for specific geographic locations (syntax). For instance, forest Nenets often employ "additive pitch organization" - i.e., they insert small melodic motifs that contrast formula-like personal tune to generate "open-end" recitativelike improvisatory development in metrically flexible development - very much unlike the metrically rigid formulaic style of tundra Nenets [275].

To sum up, the existence of the institution of personal song indicates that human music naturally grew from animal calls by means of gradual accumulation of timbral, melodic, rhythmic, and metric structural features, designed to index the ever-growing synchronic (live) and diachronic (deceased) number of persons, while reflecting their family relations. The same method of auditory indexing was implemented to organize the interaction between people and supernatural entities, whose relations were modeled after human kins. Two primary biological factors behind this system were the necessity to control for incest marriages and interbreeding and the instinct to mark and reserve territory for one's posterity. The cardinality of these both issues for one's survival in harsh climatic conditions must be responsible for the imperative of honesty in the use of personal song. Once personal song was cross-culturally adopted as a reliably credible signal, honesty became the staple of music that evolved from personal song: e.g., "personal patterns" of timbre-based instrumental music, duetic forms of vocal and instrumental musicking, etc. [12].

Something similar to personal song probably constituted a transition from animal-like communication of hominins to human music in the conditions of low population density and harsh climate, when tribes had to keep moving over sizable territory in order to find resources necessary for survival. The bonding function of Samoyedic-like musicking is secondary to 5 functions of the above-listed personal forms of music and is confined mostly to child rearing [122,260]. Scarcity of population takes away a systemic need in using music to promote greater cohesion for intertribal competition. It is not the personal songs that bind people together but the kin relations that determine the similarities between personal songs.

\section{Human aggression and music evolution: some concerns}

As noted in previous sections, a lot of evidence suggests that the formation of conventions of music communication demanded the increase in contacts between individuals and stability of intergenerational cultural transmission. It is hard to imagine how these requirements could have been met without radical reduction in aggressiveness and promotion of friendly behavior and cooperation. One important reason for this is that the physiological mechanisms, involved in the control of aggression, exert their influence on the functionality of many body parts, cognitive capacities, and behaviors, involved in complex abilities such as music and language. Let us overview the issue of aggressiveness in regard to cultural evolution.

\subsection{Aggression management through the course of human evolution}

In comparison to extant primates (perhaps, with the exception of bonobos) and, presumably to extinct hominins, humans exhibit an increased prosocial behavior. The so-called "self- 
domestication" hypothesis, proposed by Hare [1], attributes this to an evolutionary process that parallels the changes experienced by domesticated varieties of mammals. The principal difference is that if animals were domesticated by humans via selection for tameness, the supposed "domestication" of humans must have occurred without the agency of a certain species - through the series of unintentional adaptations of human behavior in response to external triggering factors (hence Hare's label "self-domestication"). Its end-result is the same as taming wild animals. The emergence of neurobiological mechanisms, regulating aggression, has made humans less emotionally reactive and more tolerant of strangers - quite on par with domesticated animals. For humans, animal taming finds its analog in cultivating prosocial behaviors, particularly, in the reduction of the in-group reactive aggression.

Because aggression management engages diverse hormones, regulating many parts of the body (particularly, the hypothalamic-pituitary-adrenal axis), selection for tameness likely results in changes in many diverse physical, behavioral, and cognitive features. This is what we indeed observe in most animals, termed as a "domestication syndrome" [276-278]. Many of the features encompassing this "syndrome" - i.e., a set of specific morphological traits and behavioral patterns - can be formulated for humans. Humans differ from extinct hominins and extant primates by smaller cranial robusticity, reduced sexual dimorphism, neotenic traits (e.g., a childish face and a prolonged juvenile period), increased prosocial behavior, reduced reactive aggression, and increased play behavior [279-285]. According to the proponents of the self-domestication hypothesis, these changes have power to enable the emergence of most of our species-specific distinctive features, including our enhanced social cognition, increased cooperation, extended social networks, and ultimately, our sophisticated culture and advanced technology. Learning, teaching, and playing behaviors are known to carry a long-lasting impact on cultural evolution.

There are some important differences from domesticated animals. Most importantly, we do not exhibit a notable reduction in brain size. However, not all domesticates display the full suite of features associated with the "domestication syndrome" (see [286] for review). ${ }^{30}$ Criticisms have been also raised regarding the ultimate physiological mechanism responsible for the emergence of domestication features, particularly, the view that the latter result from a reduced input to the neural crest cells, as suggested by some proponents of the "domestication syndrome" hypothesis [276] (see [277,278] for recent discussions). Still, scholars seem to be reaching a consensus about the long-term impact of the increased tolerance on human physiology and its contribution to the human-specific phenotypic traits. These influences are attributed to direct selection against reactive aggression or to the overall preference for self-control [287].

However, we have to note that this hypothetical "self-domestication" should be regarded not as an evolutionary process brought to completion, but rather as a phenotype that can be manifested in humans in a variety of ways, depending on environmental and cultural factors. Thus, in societies where women have higher social status, sexual dimorphism is reduced as a result of a sustained female preference for less-aggressive males. This suggests that in some societies, features of "domestication syndrome" might be found intensified, whereas in others - attenuated [288]. At the same time, humans differ from such animals as bonobos (also claimed to have passed through a self-domestication process) by higher levels of proactive aggression, which tends to generally increase the frequency of conflicts with non-kin conspecifics [289]. A general increase in proactive

\footnotetext{
30 It is true that Cro-Magnons exhibited larger brains/skulls compared to extinct hominis, but it is equally true that present-day humans have smaller brains/skulls compared to archaic (modern) humans (from ca $100 \mathrm{kya}$ ). This reduction can be indeed associated with the effects of the "domestication syndrome" as defined for domestic animals.
} 
aggression is especially pronounced at the later evolutionary stages, starting from the Holocene. Supporting this view, distinctive features of "self-domestication," particularly craniofacial feminization, reached its peak at the end of Upper Paleolithic, and are attenuated in present-day populations [290]. At the same time, selected candidate genes for mammal domestication show evidence of positive selection in Europe as recently as $6 \mathrm{kya}$ [291]. The latter is a time of important social changes in Eurasia, including a notable increase in population density, the development of complex trading networks, and the spread of sedentism. These factors impacted both inter- and intra-group human interactions. The dynamic nature of aggression management throughout the last 40,000 years reinforces the view that "self-domestication" is an ongoing process in the human species, manifested differently in different populations in response to environmental variation.

Although we find Hare's concept of "self-domestication" valuable for understanding the evolution of music, his choice of this term can confuse a reader, when applied to Paleolithic cultural traits: could "self-domestication" have occurred before the actual domestication? The conventional meaning of the word "domestication" suggests not so. Also, "to domesticate" is a transitive verb: humans tamed wild animals in order to use them at home. "Domestication" of humans does not fit the same scheme and appears counterintuitive. Therefore, we propose a new, more general and accurate term - conspecific sympathy - as a shorthand for the evolutionary changes in human behavior, which entails a reduction in reactive aggression, an increase in prosocial behavior, and the physiological and cognitive consequences of such changes. This term covers the entire line of evolutionary development of our species and might be extended to include other hominins, once evidence of their aggression management is uncovered.

Judging by the currently available data, it does not seem that early hominins (Homo erectus and earlier) showed a domesticated phenotype. With regards to extinct hominins, (pro)social behavior has to be inferred from indirect evidence, mostly signs of aggression, like injuries or evidence of violent death, and from the nature of social networks. The most well-studied extinct hominin is Homo Neanderthalis. There is evidence of violent deaths among this species (e.g., one of the individuals from the Sima de los Huesos [292]), but this evidence is very limited. The rate of traumas among Neanderthals fits within the range of modern hunter-gatherer populations [293]. There is evidence that some of traumatic injuries were caused by interpersonal violence [294]. Regarding socialization patterns, we will ignore differences that could be inferred from physical features, like a brain morphology (suggestive of a social brain), as this evidence is really indirect. That said, most researchers would agree that Neanderthal networks were less extended that human networks, being restricted to narrowly local groups (e.g., [295]), although archeological remains from late periods suggest that the social organization of the last Neanderthals might have been not so simple (see [296] for discussion).

Here we should specify that the notion of conspecific sympathy does not imply the complete absence of aggressive behavior - merely the default "liking" of other representatives of the same species upon the first encounter. As we shall see below, the development of conspecific sympathy, connected to the evolution of music, is followed by an increase of proactive aggression towards selected outsiders at later evolutionary stages. 


\subsection{Possible causes for the reduction in reactive aggression: focusing on the environment and hospitality}

An intriguing question is why reactive aggression became reduced during human evolution. As noted, animal domestication was achieved by humans through selective breeding for tameness, because domesticated animals provided resources important for human sustenance. Obviously, no species domesticated humans. Commonly cited factors that might have triggered a reduction in our reactive aggression are as follows [219,297]:

1) the rise of community living;

2) the advent of co-parenting as children demanded more attention during longer periods;

3) changes in human foraging ecology.

All these factors can be confidently expected to have promoted a selection toward greater tolerance for partners and extra-group individuals. To the above-mentioned factors, we wish to add yet another one that we find of particular interest for the evolution of music:

4) climate deterioration beginning ca 110 kya and ending ca 15 kya.

There is statistically robust evidence that the increased vulnerability to climate change was the primary cause for hominin extinctions [298]. On the other hand, harsher environments have been proved to favor prosocial behavior, specifically, intergroup tolerance [299]. Challenges of survival in extreme environments often correspond to the presence of special hospitality codes amongst populations that inhabit such environments. Well-known examples are indigenous ethnicities who live in high altitudes of the Caucasus Mountains [300] or within the Polar Girdle [301]. According to the implicit etiquette rules imposed by hospitality codes, any settled inhabitant is expected to provide shelter, food, and entertainment to a stranger visitor, sometimes even including the custom of lending his own wife. The vast geographic, ethnic, and cultural differences between the Caucasus and Chukotka lead us to believe that such customs are not culture-specific but constitute a sociobiological adaptation to life in extreme environments.

Usually, hospitality codes are authorized by ancient deeply-rooted religious beliefs. Thus, numerous Caucasian ethnicities, such as the Adygeis, the Chechen, the Ingush, and the Nogayans, have proverbs like "Guest is the messenger of God," or "The household that is poor in guests is cursed," and serve their guests by entire families no matter how hard this service is [300]. A traveler can ride through the entire country without a penny, enter any house without asking permission, and get a shelter, a bed, company, food, music, money, and assistance in his business - without being asked a question about his identity and whereabouts. In fact, a special guestroom or guest-house is reserved by each mountain family for this purpose. This custom seems to be very ancient: Xenophon describes it in his treatise "Anabasis" (c.370 BCE).

Remarkably similar is the hospitality of the ethnicities that populate Siberia, Russian Far East, Canada and Greenland. Eskimos and Aleuts [302], Chukchi [303], Yukaghirs [235], Orochi, and Maritime Koryaks [301] have been reported to have a custom of providing a traveller with a room and board, presenting gifts, and even offering host's wife, daughter, or an unmarried female relative (widow) to the guest to sleep with - known in literature as "hospitality prostitution." The earliest account of it was documented by the Russian merchant, Krashennikov (1819), and the missionary, Veniaminov (1823) [301]. Such wide distribution of this custom between ethnically distant ethnicities suggests its environmental origin. Only the Eskimos and Aleuts belong to the 
same language family; although the Chukchi are genetically close to Aleut-Eskimo populations, they occupy the lands much farther to the West, and their language belongs to the different family (Chukotko-Kamchatkan); the Yukaghirs originate from more distant southern territories, and their language relates to Uralic languages; the Orochis constitute the Southern group of Tungusic languages and originate from the Amur River region, further south; finally, the Koryaks inhabit Kamchatka, speak the language related to Chukchi, and are considered to genetically relate to the Sakhalin Nivkhs.

Even wider distribution of the hospitality code that also included "hospitality prostitution" is suggested by the multiple references to the combination of sex and hospitality in sacred Vedic texts [304]. The foot-washing ritual seems to symbolize the transformation of the visitor's semen into the fertilizing force, returned to the host's wife, as a sacred purification of a household and the establishment of a "blood relation" between the honored guest and the host's family. The IndoArian roots of such ancient hospitality ritual might explain its historic prevalence in the Mediterranean area and the Caucasus [305] - probably spread around by the cultural influence of the Ancient Greek civilization. Nevertheless, the customs of Arctic peoples can hardly be explained by the Indo-Arian cultural influences, although the classic Greco-Roman sources come surprisingly close to the explanations of the rationale behind the hospitality customs told by Arctic inhabitants.

Eskimo informants are consistent on connecting hospitality with violence: when two unfamiliar indigenous hunters meet in the tundra, their first impulse is to run away, or, if this is impossible, to kill first before being killed - however, this impulse faces the immediate objection: whenever a hunter does not return home, his clan assumes the murder and starts looking for a potential murderer who might have been within the hunting range [306]. Suspicions and resentments are passed from generation to generation, and vengeance is considered a virtue, which poses a real danger for a murderer and his clan of a retribution from the victim's clan. The most effective protection is seen not in violence but in building a network of relationships to reduce the number of "strangers". Hennigh gives an example of a battle between two Eskimo clans averted by one man running back and forth between two confronting lines, shouting that he did not know which side to join because of seeing relatives in both of them. Since the relationship in tribal societies is defined in terms of kinship, the easiest way of establishing relation to an unrelated person is by offering that person one's own wife. For this reason, the practice of wife-trading usually engaged partners from socially distant and potentially dangerous families (ibid.). ${ }^{31}$

The Indo-European tradition closely follows the logic of lumping hospitality and hostility together - the same Proto-Indo-European root *ghos-ti meant 'stranger', 'guest', and 'host' and evolved into three Latin roots: hostis ('enemy'), hostia ('sacrifice') and hospes ('hospice', 'hospitality', and 'hospital') [305]. The law of hospitality, adopted by the Greco-Roman tradition (aka Greek philoxenos - 'love of strangers', or, xenia), aimed at protecting the host from a potential attack by the stranger through the reciprocity of demonstrating the host's will to protect the stranger (ibid.). Strangers were feared, since their intentions were unknown, so they often appeared as bearers of magical power. Hence, the association of hospitality with honoring the Gods, common for Eastern

\footnotetext{
31 This line of reasoning is highlighted in Bogoraz' anecdote about the Chukchi social organization [303]. During a journey through the Kolyma district, the chief imperial officer Karzin accepted the "hospitality prostitution" offer from a wealthy Chukchi reindeer-breeder. After Karzin's return to Sredne-Kolymsk, his housekeeper threw a feast, to which the very same reindeer-breeder was invited. The latter got drunk and started throwing off one piece of cloth after another until becoming naked. When the host told him to go to sleep, this Chukchi replied: "but you must allow me to sleep with this wife of yours, just as you have slept with mine" (p.607).
} 
Mediterranean traditions of Mesopotamia, Asia Minor, Syria, Palestine, and Egypt. The oldest document capturing this association is the 3,500 BCE text "Teaching of Khety" [307]. Greeks considered the top God of their Pantheon, Zeus, the patron of strangers (Zeus Xenios) and believed that gods often traveled in disguise to check if people observed xenia, rewarding those who did and punishing the offenders.

Homer's epics, regarded by scholars as documentation of the tribal organization of the Archaic Greek culture of the Iron Age, especially the Odyssey, can be regarded as the study of the law of hospitality. The latter is repeatedly used in the Odyssey's fabula as a type-scene - i.e., a recurring situation, narrated according to a fixed pattern. Therefore, Homer's epics present the possibility to abstract and investigate the distinct cultural elements that characterize xenia [308]. The traveler was usually on some mission, to which the host was expected to help (provided the guest chose to disclose his circumstances). The host celebrated the guest's arrival, arranged for his meal, bath, and sleep, offered gifts and a departure meal. In many stories, gods rewarded the host. In essence, the formal recognition of a guest-friendship relationship constituted a ritual of establishing pseudokinship relations between two persons from unrelated clans, sanctioned by the exchange of gifts [309]. The value of this ritual is disclosed in the descriptions of the corruption of hospitality, e.g., the episode of xenoktonos ('slaying of guests') by Cyclops in the Odyssey. Cyclops are characterized as beast-like insolent, lawless, impious, barbaric, and uncivilized ("each one is a lawgiver to his children and wives"), therefore punished by gods.

In this light, it is not surprising that xenia obtained the status of a law in Greek poleis. Plutarch reported in his "Vita" that Solon, the first Greek lawgiver of the 7th century BCE, held xenia as a prime virtue. Plato specified the rules and criteria for implementing xenia in his "Laws". Aristotle, in his "The Athenian Constitution", discussed the obligations that stemmed from the ties, established through hospitality, including military alliances between households. Such alliances must have been responsible for the hereditary implementation of hospitality: host and guest exchanged with "tokens" that thereafter could be passed down between their heirs and close friends, guaranteeing the same treatment as that enjoyed by the original xenia parties [305].

Remarkably close to Greek texts are the sacred Judaic stories. Abraham, Joseph, and Moses observed hospitality customs, similar to xenia, as opposed to Egyptians. The latter are portrayed as inhospitable barbarians disrespectful of God and laws, and punished for this. Even more severe is God's punishment of the tribe of Benjamin whose corruption of hospitality caused a civil war, according to the Book of Judges [309]. Moreover, hospital Jewish patriarchs are presented as humanity-loving, whereas inhospitable Egyptians as misanthropic. The type-scene of a deity, descending to the world disguised as a traveler, receiving hospitality or inhospitality, rewarding or punishing the host, and finally revealing his identity, is pervasive for the literature of the ancient Mediterranean world (ibid.). Caucasian hospitality customs also share most of the xenia traits: the opposition of hospitality and vendetta laws, the dichotomy of war and peace, the connection to deities, the formation of hereditary ties between the host's and the guest's families [300]. Even such details as the practice of reserving a dedicated room to a guest is reminiscent of the Ancient Greek tradition - after all, the Greek term xenia literally meant 'hospitality', 'friendliness', and 'guest room'.

Anthropologists identify the basic function of hospitality as an attempt to establish a relationship between previously unrelated individuals by means of constructing a moral universe to which both host and guest agree to belong, which is embodied in the material and symbolic ritual of 
exchanging of goods and services [310]. Once the relationship is established, hospitality maintains its moral framework by consolidating the recognition that hosts and guests already share (ibid.). For this reason, hospitality is structurally transformative in its nature: it "converts strangers into familiars, enemies into friends, friends into better friends, outsiders into insiders, non-kin into kin" - "across a wide variety of social systems" (p.19). The formative social power of hospitality is particularly instrumental for maintaining social order in societies that lack hierarchical systems of authority. Members of hunting and gathering societies routinely leave one group and join another, which poses a constant dilemma between hostile and hospitable behaviors, and favors hospitality as the primary means of supporting "peace in the feud" [311]. That is why hospitality laws are as important for renewals, growth, and reproduction of heterarchical societies as the institute of marriage [310].

All of the above suggests that the origins of hospitality go way back into our prehistory. Thus, there is no reason to discard the possibility that the intermarriage between the early humans, the Neanderthals, and the Denisovans occurred, as far as humans were concerned, within the "hospitality prostitution" model, defined through the dichotomy of hospitality-hostility - following the rationale described by modern Eskimo hunters (see above).

\subsection{The origins of hospitality and its relation to kinship}

The genetic research during the last few decades brought abundant evidence of widespread admixture between the Neanderthals, the Denisovans, and the early humans by the end of the Paleolithic. All present non-African human populations outside of Oceania have a Neanderthal admixture proportion of 1.8-2.6\% - most of which appears to come from the Neanderthal populations that diverged from the Vindija Neanderthal (Croatia, 52 kya) and Mezmaiskaya-1 Neanderthal (Caucasus, c.60 kya) before their divergence c. 80-100 kya [312]. The earlier Altaic Neanderthal from the Denisova Cave, dated 122 kya, diverged from the Vindija Neanderthal's lineage around 145-130 kya, but did not carry the gene flow to modern humans (ibid.). The fourth recently analyzed Neanderthal genome from Chagyrskaya Cave (Altai, $106 \mathrm{~km}$ west of Denisova Cave, $80 \mathrm{kya}$ ) is closer to the Vindija Neanderthal (52 kya) and European Neanderthals who lived $\sim 50$ kya than to the Altaic Neanderthal (122 kya) [313]. This and the similarities of the artifacts, found in Chagyrskaya Cave, to the artifact assemblages in central and eastern Europe suggests the eastward expansion of the Neanderthals (ibid.). Hublin proposed the eastward expansion of Western Neanderthals (from such "classic" Neanderthal sites as Ehringsdorf) via the Near East towards Central Asia (Teshik-Tash and Obi-Rakhmat), where the warmer climate made eastern Neanderthals differ from Western ones anatomically and culturally [314].

Denisovans are an extinct hominin species or subspecies discovered in the Denisova Cave in Altai in 2008 and named after the cave that was occupied 280-30 kya [315]. The nuclear DNA of the phalanx and tooth of 2 individuals from the same population group showed that Denisovans constituted a sister group to Neanderthals that diverged from them after modern humans diverged from Neanderthals, around $800 \mathrm{kya}$ (ibid.). The analysis of a high-coverage genome (30x) obtained from the same fossil confirmed that date and revealed that Denisovans have contributed to the genomes of modern Melanesians (4-6\%, about twice higher than the Neanderthal DNA in East Asians), Australian Aborigines, and Southeast Asian islanders, while being virtually absent in Europeans [316] and very low in East Asian and American populations [317]. However, there was a significant Denisovan genetic contribution to archaic humans: a 34-kya-old woman from the 
Salkhit Valley in Eastern Mongolia, who had a Denisovan ancestor about 42 kya, and a 40-kyaold Tianyuan man from the Tianyuan Cave near Beijing [318]. Recently, ancient mitochondrial DNA from Denisovans was detected in the sediments from Baishiya Karst Cave on the northeastern edge of the Tibetan Plateau, Gansu, China, indicating the Denisovan presence at about 100, 60, and possibly 45 kya [319]. The presence of a Denisovan EPAS1 gene that aids highaltitude survival in modern-day Tibetans suggests that Denisovans were mountain-dwellers [320]. The nuclear DNA sequence diversity among the Denisovans exceeded that of Neandertals, but was much lower than among present-day humans, which indicates that Denisovans were more successful than Neanderthals in adapting to the harsh Siberian climate - persisting in the Altai region for at least 60,000 years [321].

Admixtures amongst Late Pleistocene hominin groups was likely common when they met, albeit infrequently - only humans had an opportunity for repeated interactions with archaic groups over a wide geographic range due to their greater density of population [322]. Specifically, the Altai region was a contact zone between Denisovan and Neanderthal populations throughout much of the Middle Paleolithic, so that Western Neanderthals had little to no Denisovan ancestry [323]. The Altai region was also the contact zone for the earliest humans who just left Africa to meet and interbreed with the eastern Neanderthals ca 100 kya: there is evidence of the gene flow from early humans to Neanderthals and no such evidence for the European Neanderthals [324]. Modern East Asians are genetically substantially more similar to Neanderthals than modern Europeans - despite the fact that no Neanderthal fossils have been found in East Asia [325]. This suggests that there were hybrid human/Neanderthal populations migrating from Europe eastward. The alternative to the Altai region of possible crossbreeding could be Levant: the human sites at Skhul and Qafzeh and a Neanderthal settlement at Tabun are dated 120 kya [324]. The dating of contexts associated with the Neanderthal remains at Tabun, Dederiyeh, Kebara, and Amud Caves shows that Neandertals still occupied the southern Levant during 80-55 kya [326].

Interbreeding of Neanderthals, Denisovans, and early humans testifies that conspecific sympathy indeed existed ca 110-40 kya, and the interaction between these different species involved the increase in prosocial behavior - perhaps, even favored prosocial behavior on part of the humans. Since we have no evidence of reduction in aggressive behavior amongst Neanderthals and Denisovans, and human populations generally exceeded their populations, it is reasonable to ascribe the interest in inter-specific contacts to humans. It is possible that Neanderthals and Denisovans reached the evolutionary stage of proto-music and proto-language - like humans. This stage was still characterized with relatively high levels of reactive aggression. Then, ongoing interbreeding between all 3 species must have brought these levels down at least in some social contexts. Plausibly, their interaction might have been regulated by some kind of hospitality norms.

What underlies hospitality is the notion of kin, which determines the discrimination between "relatives" and "strangers. Unfortunately, the time of emergence of kinship can hardly be answered with certainty due to the difficulty of defining the material manifestations of kinship in archeological contexts [327]. However, the hominin admixtures during the Middle Paleolithic (particularly, between Neanderthals, Denisovans, and modern humans) presented a fertile ground for the emergence of practice of tracking the ancestral lineage within a community, since different hominins visibly differed in their physical appearance. Accordingly, posterity of Neanderthalhuman and Denisovan-human hybrids certainly differed from non-hybrids, which would have provided the most obvious demonstration of kin relations to all tribe-members, promoting the distinction between a "relative" and "non-relative" within a tribe. 
Gowlett, in his overview of the available data on the genesis of kinship, concluded that there was a good chance for the core elements of kin-like system to have emerged sometime 150-100 kya as a result of "not-quite-total replacement" of older hominin populations [327]. The time period of 45-35 kya in Eurasia brought about the genetic separation between European (e.g., Kostenki man, 36 kya) and Asian (Tianyuan man, $40 \mathrm{kya}$ ) human populations, which likely occurred prior to 40 kya, but was complicated by their subsequent admixtures [328]. The Gravettian culture in West and Central Europe marked the emergence of population structure in distinctly European population that persisted throughout 34-26 kya, overlapping with the Aurignacian culture (associated with the 35-kya-old Goyet Q116-1 individual), which probably represented even earlier structured European population (ibid.). It would be strange if physical and cultural differences between such populations, sustained over thousands of years across vast geographic regions, would not have put in place at least some sense of ethnic identity for their members. Thus, personal ornaments discovered in Gravettian burials might have functioned as "ethnic badges" to differentiate social groups [329]. Population stratification - i.e., a systemic differentiation of allele frequencies between subpopulations as a result of non-random mating - combined with cultural conservation, was likely to promote the discrimination between different population strata. The formation of a system of social identification prototypical of clan membership might have been the outcome of such discrimination.

As Gowlett rightfully emphasizes, the crucial factor that sets apart human kinship versus basic parent-child ties, observed amongst chimpanzees, is that such ties carry little importance in daily affiliations of chimpanzees. This difference seems to have origins in the difference of environmental adaptations: chimpanzees live in large groups in tropical forests, while hominins lived in smaller groups in more open-space environments that required long-distance ranging and seasonal movements [327]. Human hunter/gatherer populations often form relatively large communities, but their members cannot always be together, since food supply rarely permits it. The necessity to account for membership in smaller groups, specializing in specific types of hunting and gathering, makes kin-like affiliations important for human sustenance. Life in harsh climatic conditions further pressurizes hunters and gatherers to form small-group alliances and frequently change membership in them - which is likely to promote hospitality towards new members due to the collective incentive of mutual cooperation. Hazards of long-distance ranging across vast desolate territory make the strategy of helping strangers viable for mutual survival through reciprocal aid, as opposed to the strategy of fighting for limited resources and facing the possibility of death, crippling trauma, and revenge of the stranger's relatives. Long-term commitment to the strategy of default aid, unless the reciprocity is violated by the other party - on the "tit-for-tat" basis - enables ritualization of the display of friendliness and cooperation between unrelated persons into a hospitality code.

The establishment of territorial borders between Neanderthals as "the people of the north", Denisovans as "the people of the east", as Gowlett notes [327], and early human newcomers from Africa as "the people of the south" increased the probability of their regular interaction at the crossroads somewhere around Central Asia and Altai, preparing the ground for the emergence of hospitality and kinship - and posing the need to signal the membership in a specific tribe. Gowlett sees language as a primary tool for labeling individuals within groups and inventing words for classes of relationship. Gowlett believes that the emergence of kinship was hardly possible without language. However, as we demonstrated above, the institution of personal song fulfills the purpose of social identification in a much more intuitive, cross-cultural, and effective way than linguistic 
names. In fact, personal songs do execute the role of "lingua franca" across the enormous range of northeastern Eurasia. That said, as we will discuss in detail in the next section, we also grant language a key role in modifying these social dynamics, particularly during the Upper Paleolithic, and specifically, under the effects of changes in aggression management as features of selfdomestication increased.

It seems that the more logical evolutionary scenario is not the simple 2-stage succession of protokin/proto-language by rudimentary kin/language, as held by the Barnard's model [330], but a much more complex development:

1) Proto-kinship introduces inclusive kinship;

2) Proto-music in the form of timbre-oriented signaling introduces hedonistic and ludic functions while elaborating and ritualizing the expressive means of communicating emotional states, elaborating call systems typical for social animals;

3) Human proto-language (to be distinguished from the "proto-languages" purportedly used by other species) spontaneously expresses one's affective state through interjections, while developing the capacity to convey referential meaning through onomatopoeia;

4) Proto-kinship evolves into rudimentary kinship that introduces the antithesis "us-them" as a family group within a community;

5) Pre-music (transitional form from proto-music to music) in the form of timbre-oriented personal song supports social identification and introduces recreational and inter-personal bonding functions, leading to the emergence of music;

6) Human pre-language (transitional form from human proto-language to full-fledged languages) capitalizes on the bonds, established and supported by personal song, and reinforces deictic referential communication by single words and simplest phrases.

Here we have to underline that the southern border of the Eurasian community of indigenous ethnoses that retain personal song falls on the Altai-Sayan region (Kamasins and Kotts) - the same that housed Neanderthals, Denisovans, and early humans (Fig.5). The institute of personal song very well could constitute an enormously long non-interrupted cultural tradition, sustained by the extremely stable harsh continental climate, geographic isolation by the high altitude, very low density of population, and life full of hazards. Such conditions make the strategy of landholding not viable, causing ongoing migration over large territory by small "packs", each of which has to share this territory with others between the neighboring tribes, helping each other to survive and regulating the mutual use of shared resources [331]. This is the context within which personal song acquired the functionality of regional lingua franca.

\subsection{Kinship and personal song}

As we already pointed out, one of the sociobiological functions of personal song is identifying the ancestral lineage, beneficial for avoiding the incest. This capacity was probably forged by "trial and error," observing the detrimental outcomes of incest in depopulated tribes, and carried even greater importance in lives of hominins compared to modern Siberians due to even smaller population density. This aspect of personal song also complements the "hospitality prostitution", which effectively counters incest by supporting the genetic flow into a kin from strangers. Thus, personal song introduces an important upgrade from territorial animal calls. Contrary to a 
widespread conviction that incest avoidance is peculiar to humans and relies on taboos established by cultural conventions, primatologists have convincingly made a case for apparent constraints on sexual relations within nonhuman primate groups [332]. The origin of incest avoidance cannot be explained by social taboos: at least some forms of psychologically motivated incest avoidance are primate homologies - most clearly, those between mothers and offspring and between maternally related siblings (ibid.). What distinguishes human incest avoidance from that of primates is the widening of a circle of close relatives and their abstraction into the concept of consanguinity that transforms the individual-level reaction of sexual aversion or indifference to a closely related person into the group-level morally-based prohibition. Both "hospitality prostitution" and personal songs support the observation of social restrictions on closely consanguineous sexual relations, which suggests that personal song emerged after the institution of hospitality codes and spontaneous discovery of "hospitality prostitution".

The remarkable similarity of the institute of personal song in large parts of Asia and North America, agreeing with the evidence of gene transfer to their present-day populations, as well as the importance of personal song for the ancestor cult and marriage customs all suggest that personal song was exported from Siberia to Alaska (Fig.5). The decline in global sea levels during the Last Glacial Period created a land bridge, the central portion of which is known as Beringia, which connected Asia and America and remained traversable between 30-12 kya [333]. The closest to this bridge Paleolithic archeological sites on the Asian side are the Buor-Khaya (45 kya) and Yana RHS (31 kya) settlements, both clustered near the Yana Bay of the Laptev Sea, Yakutia [334] and inhabited until $22 \mathrm{kya}-2,200 \mathrm{~km}$ away from the Bering Strait. On the American side, the closest is Swan Point, Alaska (14 kya), $650 \mathrm{~km}$ away from the Bering Strait [335]. However, the first people definitely arrived in America earlier than $14 \mathrm{kya}$, since the oldest pre-Clovis sites are dated $15.5 \mathrm{kya}$, including Monte Verde in Chile [336] and it would take many centuries to migrate all the way from Alaska to Chile. It seems that ca 20 kya would be a plausible date of the beginning of peopling North America.

The genetic studies are quite conclusive in showing that Native Americans have only Ancient North Siberian and East Asian ancestry, which both had gene flow between one another (ibid.). The comparative genetic analysis of the ancient DNA reveals that the first Yana inhabitants were Ancient North Siberians (now extinct) who diverged from Western Eurasians (e.g., Sunghir man, Vladimir, Russia, 32 kya) about 38 kya, soon after the latter split from Ancient East Asians (Tianyuan man, China, 40 kya) [258]. Ancient North Siberians were largely replaced by East Asians (e.g., inhabitants of the Devil's Gate Cave in Primorskoye or the Bianbian Cave in Shandong) between 20-11 kya, giving rise to ancestral Native Americans and Ancient Paleosiberians, represented by a 9.8 kya skeleton from Duvanny Yar, near the Kolyma River (ibid.). The latter represents the closest relative to the ancestral Native American population. His ancestors diverged ca 30 kya from East Asians (ibid.). Ancient Paleosiberians were ancestral to modern Koryaks and Itelmen.

Ancient North Siberians either migrated to southeastern Beringia via the coastal corridor along the Sea of Okhotsk and admixed with Early East Asians, or, admixed in the Baikal region and expanded northwise during the Last glacial Maximum (ibid.). The 24-kya-old Mal'ta boy from south-central Siberia (and even more so the Afonova Gora woman, 17 kya, from the neighboring Altai-Sayan region) had the mitochondrial genome of haplogroup $U$ (that was also found at high frequency among Upper Paleolithic European hunter-gatherers) and the Y chromosome that was basal to modern-day western Eurasians [337]. This testifies to the far north-eastern distribution of 
western populations. About 14-38\% of Native American ancestry may originate through the gene flow from this ancient population (ibid.). The newly sequenced Upper Paleolithic genome from the Ust-Kyakhta-3 site, south to the Lake Baikal, is close to the Duvanny Yar individual and, like the latter, is shifted toward Native American populations [338]. The entire area from Baikal to Yana and Kolyma during 24-9 kya probably was occupied by Ancient North Siberians prior to the ethnogenesis of Ancient Paleosiberians.

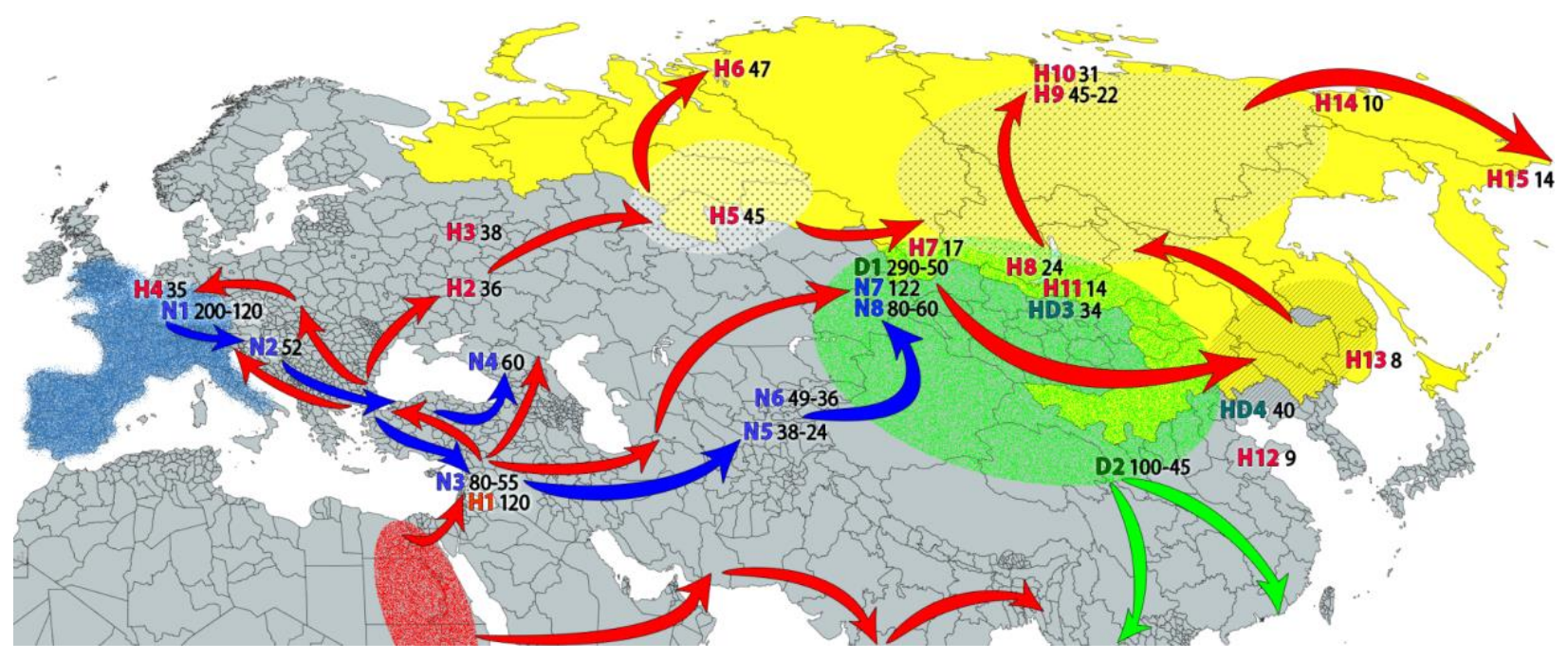

Figure-5. The major migration pathways in Eurasia c.290-7 kya plotted against the geographic location of personal song. The yellow color indicates the areas inhabited by those indigenous ethnicities that retain the tradition of personal song: Nenets, Komi, Mansi, Khanty, Selkups, Kets, Kamasins, Kotts, Enets, Nganasans, Yakuts, Evens, Evenks, Nanai, Udeges, Ulchi, Nivkhs, Negidals, Yukagirs, Itelmens, Chuvans, Koryaks, Kereks, Chukchi, Eskimos, Aleuts, and Ainus (territories of Russia, Mongolia, China, and Japan). The grainy blue filling marks the area in Europe, originally populated by Neanderthals, and the dark blue arrows show their eastward migration. The numbers reflect the sites mentioned in this paper: the colored number reflects the order along the migration route, whereas the black number to the right indicates the date (in kya). N1 - Ehringsdorf, N2 Vindija, N3 - Kebara, N4 - Mezmaiskaya-1, N5 - Teshik-Tash, N6 - Obi-Rakhmat, N7 - Denisova, N8 - Chagyrskaya Cave. Grainy green filling encircles the hypothetical area of Denisovan origin, and green arrows show the possible direction of their migration towards Melanesia. D1 - Denisova, D2 Baishiya Karst, HD3 and HD4 - the early humans with Denisovan ancestry: respectively, Salkhit and Tianyuan. The grainy red filling encircles the area in Africa from where the early human exodus started [339]. H1 - Tabun, H2 - Kostenki, H3 - Sunghir, H4 - Goyet Q116-1, H5 - Ust'-Ishim, H6 Sopochinskaya Karga, H7 - Afonova Gora-3, H8 - Mal'ta MA-1, H9 - Buor-Khaya, H10 - Yana RHS, H11 - Ust-Kyakhta-3, H12 - Bianbian, H13 - Devil's Gates, H14 - Duvanny Yar, H15 - Swan Point. Arrows reflect the regional traditional trading routes. The dotted oval covers the hypothetical location of Ancient North Eurasians, the diagonal lines - Northeast Asians, and the combination of dots and diagonal lines - Ancient North Siberians. This figure is original.

The Sayan mountains, bordering on the Baikal, are part of the Altai-Sayan mountain system, where the Neanderthals, Denisovans and early humans intermarried, where personal song has survived along with the seok clan system, where present-day shares their DNA with the Native Americans, and from where the local indigenous population probably started long journey northeast into the New World about 25 kya. The latter is indicated by 4 shared major mitochondrial DNA haplogroups and founding Y-chromosome haplogroups of contemporary Native American and South Altaian populations [340]. Also, there is no genomic evidence that any population from a 
geographic region other than northeast Asia was an important source of the gene flow to America's first people [336]. Human arrival to Alaska most probably occurred before $12 \mathrm{kya}$, when Beringia became submerged by the waters of the Bering Strait - likely, ca 23-19 kya, when the water level was at its lowest.

The totality of available information supports the scenario that personal song emerged through the intermarriage of different structured populations somewhere around the Altai area, triggering the ongoing feedback between the general reduction in aggression and the development of social structures, music, and language, further promoted by the formation of hospitality codes. Once formed, this cultural tradition was carried to North America sometime around 20 kya.

Accounts, hypothesizing about an accidental invention of music (e.g. [10]), seem to us too general and unrealistic, because human music neither directly brings an immediate biologic benefit, nor has natural prototypes that could serve as the basis for its invention (i.e., it cannot be regarded as a direct emulation of bird songs or animal calls due to significant differences in syntax and semantics). In our view, the evolution of music became possible only once social bonds were potentiated and diversified as a result of our increased prosociality. Again, both the view that complex music evolved to support extensive human social bonding and the view that it was selected to provide increasingly sophisticated credible signals to cope with growing social conflicts of interest make sense only for societies that have achieved a threshold of complexity. Hence, we argue for a reversion of casualty: it is not music that enabled humans to use more complex social structures; it is more complex structures, brought about by our increased prosociality, combined with the global warming, that enabled the stable population growth across vast territories - which together enabled music to evolve towards greater complexity. In the next section we present a detailed account of this view.

\subsection{The contribution of prosocial behavior to music complexity}

In order for music to exercise entrainment and transposability (defining features of music), a music-maker has to already have a friendly disposition towards a partner-performer and a listener. Music performance is not going to entrain someone who is more likely to engage in a fight than in a collective musicking. Hostility is known to obstruct appreciation of music as well: listeners tend to find performance of a disliked musician faulty and meritless [341]. Often such dislike poses a barrier to fair evaluation - a listener simply does not want to listen to the disliked musician. The same avoidance attitude applies to the prospect of joining a disliked audience. Hostility has the power to obstruct entrainment and emotional contagion - two principal ways for music to carry its prosocial action.

It is true that the hedonistic function of music can potentially divert one's state of anger and irritation to some extent by inducing the experience of pleasure, thereby circumventing negative personal emotions, as argued by Perlovsky [186]. Similar effect might carry the reward mechanism in collective musicking, as proposed by Harvey [192]. The experience of togetherness and collective power in inter-tribal competition also can induce positive emotions, as described by Savage et al. [10], which might overcome previous negative emotional states. However, in order for such pacifying effects to take place, the music performance has to occur first - which is impossible without having friendly disposition between the participants in a music act. Moreover, in order for musicking to become a stable cultural tradition, the hostility and aggressiveness between all the engaged parties must be not just temporarily and partially reduced but eradicated. 
Otherwise, prospective music-makers would not be seeking an opportunity to learn music, and the transmission chain will be interrupted.

A good demonstration of this requirement can be found in human interaction with domesticated animals. A dog-owner can conduct mutual activities with his dog because of the pre-existing bonds - whereas a stranger cannot. Although such a dog is domesticated, the rapport of care and support must be established first in order for cooperation to become possible. A dog has to be treated very much like the mother treats her child - understand child's vocalizations and engage in motherese and grooming-like behaviors [342]. In fact, the lexicon of auditory signals used by humans to communicate to dogs - "doggerel" [343] comes remarkably close to human "motherese" [344]. Similarities extend to the animal lullabies in traditional pastoralist societies sharing many common traits with human lullabies and animal spells - with human magic spells, found in human communication to virtually all domestic animals [13]. What unites all of these forms of musicking by means of singing, humming, whistling, and articulating unusual sounds is the framework of establishing an intimate bond between just two parties, which lays down the foundation for building a repertory of meaningful signals as a "mini-culture," confined to a duetic format [345]. It is the sum of multitudes of such "mini-cultures" that in effect generates a musical "macroculture" of a human community.

In practice, the emergence of a continuous musical tradition and the process of evolution of music can be triggered only in a community of music-users that is already consolidated by sympathetic feelings between its members. Only then the transmission of music from one member to another will launch the process of "averaging the beauty", capable of forging musical idioms. Although the transmission chain of an already established song can certainly be sustained between individuals not bound by close relation, a certain threshold of adoption is required to elevate a personal motif from the status of "mini-cultural" duetic convention to the "macro-cultural" convention of an entire community. A personal motif viable for interpersonal use must be adopted by a number of dueting couples, akin to mother/child, in a more-or-less the same semiotic functionality, in order to turn that motif into a musical idiom capable of supporting chain transmission between unrelated people. Such accumulation of duetic uses occurs naturally within a clan, where close bonds unite multiple couples so that each of them has a chance of witnessing and emulating those patterns that proved to be effective in communication. Hence, the formative power of chain transmission is brought to life within the circle of closely related people - i.e., the idiomatic genesis is promoted by kinship. A kin structure secures the reproduction of the same pattern of auditory communication between multiple couples of individuals that are already bonded by close blood relations.

Once the musical idiom is established within a music-user community, it becomes available for combinatorial and compositional creativity of its members, including multiple kins that maintain frequent contacts. Then, unrelated people within a multi-clan community can generate new combinations of conventional musical idioms, and these combinations pass the same route of cultural transmission that we described for a stand-alone musical idiom. This process forges musical equivalents of phraseological units and lays down the syntactic foundation for a music system. The prototype of this syntactic genesis can be found in the animal kingdom. Changes in the dopamine reward system have been regularly attested in domesticated animals $[84,85]$. More importantly, changes in the dopaminergic signaling have been recently claimed to potentiate vocal learning by domesticated birds. In a nutshell, domesticated species of songbirds manifest extensive signs of positive selection in genes, involved in glutamatergic signaling that attenuates stress 
responses and results in changes in dopamine release in the dorsal and ventral striatum, ultimately, leading to more variable vocalizations and greater song complexity [346]. These findings suggest that a reduction in reactive aggression during human evolution might have resulted not only in a potentiation of the effects/functions of music, but in the structuring of music itself, favoring the emergence of more diverse musical compositions.

We have already touched upon this connection in our discussion of the contribution of the numerosity of music-users to the conservation of a musical tradition: prevalence of solitary and duetic musicking promotes conservation of music syntax, whereas prevalence of performance in front of large audiences promotes innovation and competition in creativity and expressivity via musical rhetoric. When musicking is restrained to mostly a narrow family circle, the ease of learning tends to take over the expressivity in music transmission acts. There is little need in trying to intensify or exaggerate a musical expression for long-standing partners, who have already developed their rapport. Family communication tends to be brief and simple, with strong deictic components, since all members are well familiar with each other's habits, needs, and constitutions, which enables them to grasp hints without going into explanations and details.

The ethnomusicological data generally agrees with this generalization. Music traditions of minority ethnicities that maintain traditional lifestyle in sparsely populated territories, where kin and family remain the principal forms of social organization, tend to have music systems that use fewer pitch classes or are timbre-oriented. Such systems are substantially more limited in compositionality and combinatoriality than music systems of large nations, characterized by widespread and complex interactions between multiple populous social and ethnic groups. Thus, in North America, the Arapaho, Blackfoot, Cheyenne, Chippewa, Creek, Crow, Dakota, Flathead, Hidatsa, Kiowa, Mandan, Menomini, Muscogee, Omaha, Paiute, Pawnee, Shoshone, Sioux, Tutelo, Ute, Washo, and Yuchi use pentatonic and tetratonic music systems [347,348]. The Klamath, Modoc, Navajo, Creek and Yuchi use tritonic ${ }^{32}$ and even ditonic songs. In Northeastern Eurasia, ditonic modes are common amongst the Taz, Yukaghirs, Chukchi, Selkups, Yakuts, and Dolgans; tritonic modes amongst the Taz, Nganasans, Nenets, Yakuts, Yukaghits, Yughs, Oroks, Evens, Evenks, Khanty, Altaians, Kets, Kereks, Nivkhs, Nanai, Dolgans, Tofalars, Chukchi, Dolgans, Byriats, Chelkans, and Selkups; and tetratonic modes amongst Nganasans, Nenets, Nivkhs, Evenks, Taz, Nanai, Shoes, Yughs, Yukaghirs, Chukchi, Altaians, Tuvans, Buryats, Tofalars, Telengits, Chelkans, Yakuts, Khanty, Tubalars, Selkups, Eskimos, Kets, Khakas, Kamasins, Kereks, Koryaks, Enets, and Mansi [138]. Ditonic, tritonic, and tetratonic modes are encountered amongst indigenous people of the Volga Plato, such as Mari [349]. Majority of the European Arctic Sami songs are tetratonic and pentatonic [350]. Greenlandian Eskimos have a tritonic tradition [351], whereas Alaskan and Canadian Inuits use pentatonic and tetratonic songs [352]. The tribal peoples of India, such as Juang, Bhuyan, and Adi, especially in the northeast, use predominantly tetratonic, tritonic and ditonic monodic music, in sharp contrast to the rest of India [353]. Ditonic modes occupy an important place in the Vedda music of Sri Lanka and indigenous population of Tierra del Fuego [354]. Tritonic modes prevail in Maori music, followed by tetratonic and ditonic modes [355]. Pentatonic, tetratonic, and occasionally tritonic modes are typical in Polynesia [356]. The Ye-anim, Marind-anim, Kanum-anim, and Gad-ze peoples of New Guinea often use tetratonic modes, and the latter also use ditonic and tritonic ones [357]. Ditonic and tritonic music characterizes traditions of Eastern Polynesia [358].

\footnotetext{
32 The term "tritonic" here is used in reference to the tonal organization that consists of 3 pitch classes, following the paradigm of monotonic, ditonic, tetratonic, pentatonic, hexatonic, heptatonic, and octatonic - and not in reference to the musical interval of a tritone.
} 
This by no means complete list of ethnicities that use ditonic, tritonic, and tetratonic music demonstrates the wide spread of simple and well-conserved music systems, cultivated largely within tribal societies with simple and stable social structure. Noteworthy is the geographic distribution of such music that mostly coincides with the indigenous populations of Asian genetic heritage. Without going into the details of tonal organization that characterizes its simplicity, it is easy to demonstrate how greater number of pitch classes contributes to complexity of music. If an adherent of a ditonic tradition has only 2 possible combinations of tones for constructing motifs and melodies, a tritonic tradition provides a music-user with 6 combinations, tetratonic - with 24, pentatonic - with 120, heptatonic diatonic - with 5,040, and chromatic - with 479,001,600! It is pretty obvious that cultivating atonal dodecaphonic music imposes an immense cognitive burden - in polar opposite to ditonic and tritonic music.

Comparing the number of building elements for music provides a convenient universal method to estimate relative complexity of music. Humans are biologically limited in the amount of information that can be received, processed and remembered. Its threshold has been established at seven "chunks" plus/minus two, where "chunk" is a set of items treated collectively as a single unit [359]. The bottleneck of chunking is determined mostly by working memory, hence the only workaround is to compress more items into a single set, which can be done in series, reaching higher order hierarchical levels - chunking the chunks as long as they are treatable as a single unit. Chunks of a "maximally compressed" code afford the limit of 4 plus/minus one [360].

The semiotic implications of chunking are that in order to support compositional and combinatorial semiosis, a musical phrase cannot have more than 9 elements or 5 meaningful compound units. For the aspect of melody, an element is a dyadic melodic intonation (i.e., a combination of 2 pitchclasses), whereas the simplest compound unit is a melodic motif (usually, 2-5 intonations) [142]. So, the task of consulting the meaning of the constituent intonations for grasping the meaning of a musical phrase without resorting to compression requires the presence of no more than 9 different intonations per phrase. Systemic use of more than 9 intonations per phrase necessitates the "chunking" of chunks - the systemic use of compound structures, i.e., motifs, which introduces hierarchical organization and syntactic rules.

Only ditonic ( 2 possible intonations) and tritonic (6 intonations) music systems support melodies without syntax. Tetratonic music might permit individual users to use no syntax, provided they do not engage more than 9 different intonations per musical phrase. However, for the entire music community, this option is hardly available: the genesis of a repertory of melodic idioms involves all community members, and it is simply inevitable for a music system that affords 24 intonations to sooner or later have a substantial number of users using melodic idioms that exceed 9 intonations. Yet another source of complexity is rhythm - phrases are rarely restricted to a single rhythmic value and, as a rule, use rhythmic figures that consist of 2 or more rhythmic values. But each rhythmic figure constitutes a "chunk" in addition to a melodic "chunk". Even greater complexity emerges in multi-part music, where each of the parts can have its own "chunks", and the harmonic progressions formed by the integration of parts also add chunks - this time harmonic.

This important point explains the preference of indigenous ethnicities of the sparsely populated North for ditonic, tritonic, and tetratonic music in monophonic arrangement and illustrates the observation of Alekseyev that these populations prioritize musical conservation in musicking for oneself (and a few closely related persons) over innovation in musicking for many others [136]. 
This strategy allows them to maintain their music systems virtually intact over long periods of time despite powerful foreign influences and the entropy of communication.

It makes sense to distinguish these simple music systems from more complex ones by adopting the linguistic distinction between esoteric and exoteric languages, which, in turn, relies on the distinction between two basic types of societies: close-knit and open. Ongoing research in the domain of evolutionary linguistics (e.g., [361-365]), suggests that the languages spoken by small, isolated groups, organized in close-knit social networks, show distinctive structural features: large and complex sound systems, intricate morphologies with pronounced irregularities, reduced semantic compositionality, and limited syntactic complexity. Such languages are called esoteric (after [362]) and are characterized by great context-dependency, since sharing considerable amounts of knowledge via esoteric communication requires pointing to objects, their features, and actions that are directly observable for all communicating parties (see [225] for details). Most present-day hunter/gatherer societies follow this socio-linguistic model that especially suits isolated small kin-based groups. In a similar vein, the simplest forms of music systems can be called "esoteric" in reference to their limited availability to those outside of their environmental and cultural frameworks.

In contrast, languages spoken by larger groups that form more complex social networks with higher rates of cultural exchange (i.e., open societies), exhibit quite opposite features, aimed at effective communication of an increasing amount of information to support collective execution of technologically demanding tasks. Beneficial results of such tasks, in turn, promote the accumulation of knowledge to maintain newly discovered methods of sustenance. The need of conveying precise information to people not sharing any common ground with a speaker forges distinctive linguistic features that are better designed for interacting with non-kin - typically addressing such issues as conflicts and alliances. Such languages are sometimes referred to as exoteric languages [225]. Pentatonic and more complex music systems might be considered "exoteric" in a similar sense of their suitability for musical communication beyond one's family via a diverse system of conventional music genres and a repertory of musical idioms, used to convey a set of culturally important musical emotions.

The tendency of both esoteric languages and music to evolve into exoteric ones is largely determined by the effects of a reduction in reactive aggression on music variability and complexity. This is because the central property of music - hierarchical structure - depends to a great extent on cortico-basal ganglia-thalamocortical structures that are shared with language [366]. The same mechanisms of increased neuronal connectivity between cortical and subcortical components that are implicated in syntactic chunking of increasingly higher orders of complexity are also implicated in the growing suppression/inhibition of reactive aggression [367]. As argued by Benítez-Burraco and Progovac [368], a reduction in reactive aggression entails an increased cortical control over subcortical structures, supporting the advance in processing hierarchical structures not only in the domain of language, but also of music - possibly, in the process of their coevolution. Initial grammars were probably used for conveying mostly affective information, much like music - but with the specialization on verbal diatribes to replace physical aggression (see [369] for details). The resulting reduction of harm was likely to further promote the spread of verbal structures, required to express verbal aggression, leading to a greater control of subcortical networks by cortical devices. In turn, this was likely to support more complex hierarchical linguistic structures - engaging reduced aggression and language complexity in a virtuous circle. 
Similar interaction could be hypothesized for the interaction of mechanisms engaged in processing of music structures assigned to expression of anger. Perhaps, the best showcase is the treatment of aggression in Ancient Greek music culture. Violence was considered a very negative factor, jeopardizing the very foundation of civic life: "friendship amongst people" was described by Aristotle as a force that "binds the city together" [370]. Aristotle defined violence as an impulsive action upon the triggered onrush of anger, often against the internal interests of the actor [371]. Greek art music was overall decidedly non-violent and Apollonian in spirit: even the genre of martial exhortation which called to inspire citizens to go to war for the right cause, could hardly be characterized as an expression of anger, according to writings of Callinus and Tyrtaeus. One notable exception was the music of the Dionysiac cult, specifically bacchanals (not dithyrambs that were as dignified as Delphic hymns to Apollo) - performed with cymbals, drums, clappers, and bull-roarers, and accompanied by wine drinking, waving torches, and frenzied dancing [372]. Such music strikingly differed from every other Hellenic musical genre and was strictly seasonal, part of the Orphic mysteries, held annually to celebrate the fertility rite central to the grape industry.

The violent sacrifice, symbolizing the act of cutting Dionysus in pieces, was the tribute that the worshippers paid to savagery and madness against all laws that they normally abided by in their daily life [373]. The purpose of Orphic mysteries and Dionysiac cult was not to cultivate violence and anarchy, but on the contrary, to secure peace - the reverse side of the coin that had Apollo on its front. Characteristically, in Delphi, the festivals for Apollo and Dionysus followed each other every season. The opposite characteristics of Apollonian and Dionysian music were called to serve the same purpose - of ensuring order and harmony. The analysis of the depiction of Dionysiac rites on vases confirms that Dionysus performed the role of a peace-maker and the guarantor of the life-cycle's continuity by providing a controlled outlet for venting out the unwanted destructive energy [374]. Musical structures that characterized bacchanal worked as a "valve" of emotional regulation to discharge one's reactive aggression briefly, once a year, in a place, where this could not destabilize the society, in order to secure friendly disposition toward fellow citizens for the rest of the year. Subsequently, the "primitive" musical form of bacchanals was effectively isolated from the rest of the Ancient Greek music culture that greatly advanced from archaic rhapsode-like folk music [375] to highly sophisticated "Athenian modernism" [376] just in a couple of centuries. This advance in complexity of tonal organization was reflected by the numerical increase of pitchclasses engaged in the practice of music composition: from 4 pitch-classes of rhapsodic music (that corresponded to 4 lyra's strings) to 15 pitch-classes of Systema Ametabolon (see [143]).

The numerical growth of pitch-classes that characterizes the transition from esoteric to exoteric organization seems to coevolve with the progressive increase in complexity of the basic social units. The connection between the evolution of social organization and the growing complexity of music, manifested in the increase in the number of pitch-classes available to a music-maker, used to be well-established for the most part of the 20th century [39,377-380]. Starting from the 1980s, this parallel was largely abandoned in Western ethnomusicology as part of its diversion from comparative and evolutionary studies (due to concerns for political correctness in comparing more and less advanced forms) in favor of studying a single culture, while refusing to draw universalities and generalities across music cultures and restricting research to musical behavior rather than music structures [381]. For these reasons, at present, the numerical axis of the evolution of tonal organization of music is recognized only in Eastern European ethnomusicology (e.g., Sheikin [382]). Nevertheless, the collected data on indigenous music cultures of the former USSR quite convincingly (many music theorists themselves belong to local indigenous ethnicities, e.g., Eduard 
Alekseyev) supports the following cross-cultural outline of the evolution of tonal organization (Fig.6, see the summary in [383]):

1) The development of timbre-oriented modes of vocal (e.g., rasping singing) and/or instrumental (e.g., Jaw harp) music, based on matching of timbre-classes (akin to phonemes in languages),

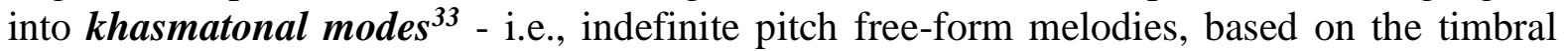
contrasts of 2-3 different registers or sound generators (e.g., nasal versus glottal) within the entire vocal range, connected by abrupt leaping; no syntax; prevalent monophony;

2) A parallel or consecutive to 1) development of ekmelic modes ${ }^{34}$ - i.e., indefinite pitch "gliding" formulaic wavelike melodies, based on the melodic functionality of 1-3(4) "floating" degrees (like pitch-levels of vowels in languages) in the expandable tessitura (not larger than an octave), enclosed within the same register, with possible timbral re-coloring, connected by smooth sliding; no syntax; mostly monophony;

3) Gradual transformation of ekmelic pitch-classes into emmelic (see the footnote No.34) and the emergence of oligotonal modes ${ }^{35}$ - 1-3 pitch classes within a narrow tessitura, not wider than about half-octave, separated by steps,${ }^{36}$ where at least one pitch class is well-defined, stable in tuning, and "consonant" in a sense of its capacity to resolve and terminate melodic motion; mostly heterarchical syntax; possible basic monody with rhythmic or single-pitch musical (proto)-instruments, gradually expanding to multi-pitch instruments (flutes) designs, but using no more than 3 pitch-classes per music-work;

4) Expansion to mesotonal modes ${ }^{37}$ - 4-6 pitch classes within a tessitura not larger than an octave, usually separated by steps, but can include a gap (leaps up to a 4th), with most classes welldefined in pitch (1-2 anchor pitch-classes supported by less-defined auxiliary classes); hierarchical syntax, usually without octave equivalence; genesis of heterophony and ensemble music, use of multiple pitches in instrumental music;

\footnotetext{
33 The term "khasmatonal" (Greek, khasma - 'hollow') was introduced by Wiora [354], in 1959, to refer to such melodies where one tone broke apart from the rest of the tones - separated with a systemically unclosed gap. This term has been adopted for the musicological analysis of those indigenous folk melodies of northeastern Eurasia that are characterized by indefinite pitch with salient leaps (4th or larger), combined with pronounced timbral modulations and/or expressive use of several contrasting registers [136].

34 The term "ekmelic" (Greek, ek - 'away' + melic - 'melodious') was used in music treatises by Cleonides, Gaudentius, and Ptolemy in reference to unclear sounds unsuitable for musical composition - in opposition to the term "emmelic" (Greek, em - 'within'), used by Aristoxenus and his school in reference to sounds with distinct intervallic structure [384]. This term has been adopted for the musicological analysis of those indigenous melodies of northeastern Eurasia that are characterized by indefinite pitch with continuous frequency modulations and/or systemic absence of fixed pitch values between the melodic tones [136]. Ekmelic modes are usually opposed to khasmatonal modes as materialization of, respectively, smoothness/similarity vs. abruptness/contrast [138].

35 The term "oligotonal" (Greek, oligo - 'few') was introduced by Kvitka [380], in 1927, to refer to musical modes that featured fewer than 7 pitch-classes, diatonic as well as non-diatonic (i.e., not conforming to the circle of 5 ths). This term was adopted for the musicological analysis of those indigenous folk melodies of northeastern Eurasia that featured discrete organization of pitch (definite pitch), while using no more than 1-3 pitch-classes, enclosed within a narrow range (about half-octave) and separated by 2nds or 3rds [138].

${ }^{36}$ The percept of intervallic "step" varies depending on the tempo, as demonstrated by Noorden: from minor 2 nd at the slowest perceptible tempo to major 3rd at the fastest [385]. Accordingly, the notion of "step" varies between different cultural traditions. The most widespread are major/minor 2nds in heptatony and major second/minor 3rds in pentatony. Some microtonal traditions can utilize steps smaller than a minor 2nd: e.g., Pamir Falak (see [386]).

37 The term "mesotonal" (Greek, meso - 'middle') was introduced by Starostina [387] in 1973, in distinction to oligotonal modes, to refer to musical modes that used 5-6 pitch classes (of diatonic and non-diatonic relations), separated by steps and small gaps (e.g., can be triad-based, like C-E-G-A, or feature the infra-fix, usually equal to perfect 4th, like C-F-G-A). However, since the majority of oligotonal melodies are characterized by the narrow range of a 3rd, and therefore feature no more than 3 pitch-classes [138], Starostina's classification should be adjusted from her original 5-6 to to 4-6 pitch classes, placing tetratonic modes into mesotony. This adjustment reflects the commonalities of oligotonal cultures with which Starostina was not familiar (she specialized in Russian folk music).
} 
5) Further expansion to multitonal modes $^{38}$ - 7-11 well-defined pitch classes, with or without octave equivalence (more than 7 classes are usually generated by adopting equivalence of the 4th or the 3rd - see [143]), featuring pronounced hierarchy of stability and instability, usually based on the contrast of even and odd degrees in a stepwise scale, 1-2 tonics (one anchor initiates a melody, while another anchor terminates it); expansion of the tessitura to a span larger than octave; complex hierarchical syntax; genesis of multi-part music; rich instrumentarium, including instrumental families;

6) The discovery of diatonic pentatony or heptatony - 5 or 7 pitch-classes of complete octave equivalence in the ambitus of about 2 octaves (maximum, 14 pitch-values of 7 pitch-classes), featuring well-defined interval classes, organized by means of the circle of 5ths; characterized by a single tonic (usually supports transposition of a melody by a fixed interval); introduction of harmonic intervals; genesis of theory of composition, rhetoric, and ethos; rise of orchestral and choral music, advance of multi-part music;

7) Gradual development of a chromatic genus within the diatonic system (Audio-25), supported by the chromatic music theory, followed by the emergence of new semantic conventions; rise of originality and authorship, the advance of organology and instrumental techniques, expansion of instrumental families and development of orchestral/choral music, leading to the extension of the ambitus over 2 octaves; increase in aesthetic appreciation, theatricality, and entertainment; the emergence of mass audiences, passive use of music starts prevailing over active use.

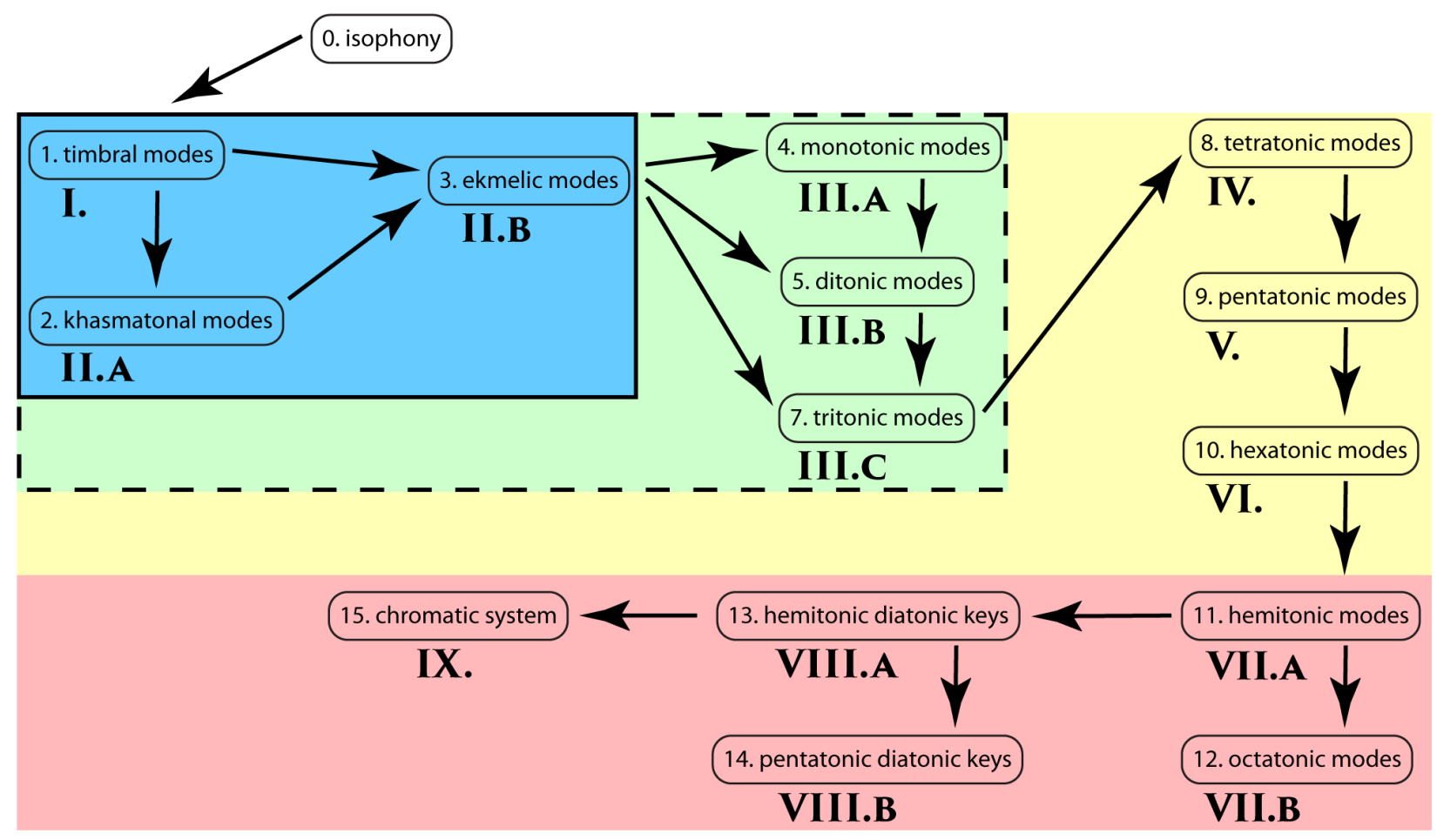

38 The concept of "multitonal" (Latin, multi - 'many') was introduced by Starostina (as polnaya diatonika - 'full diatony') [387] in 1973, in distinction to mesotonal modes, to refer to musical modes that used 7-8 (or more) pitch-classes (octave-equivalent as well as non-octave). In translating the Russian taxonomy of musical modes into English, the term "multitonal" was preferred to "polytonal" to avoid confusion with polytonality [37]. Unfortunately, systematic musicology in English has not elaborated the terminology for categorizing different types of pretonal musical modes commonly encountered in world's music. This is probably the consequence of lack of comparative analysis of indigenous forms of music (noted above). 
Figure-6. The evolution of tonal organization applicable to prehistoric and ancient music, up to ca 5th century BCE. Thin rounded rectangles with the Arabic numbers represent different schemes of tonal organization, from "isophony" that characterizes collective animal vocalizations and is hypothesized to represent proto-musical vocalizations of hominins in Africa to the emergence of chromatic music (chromatic and enharmonic genera of the Ancient Greek music). The arrows indicate the "parent-to-child" relation. The roman numbers show the chronological succession of stages, reflecting the parallel developments. The bold rectangle encloses the schemes based on indefinite pitch (i.e., no pitch-classes), the dashed rectangle - the mixture of discrete (well-defined) and indiscrete (variable) pitch classes. All the other schemes are emmelic (pitch-class based). The blue color indicates the prevalence of timbre-orientation, absence of pitch-classes, and the semiosis based on onomatopoeia. The green color marks the schemes characterized by the oligotonal addition of pitch-classes; the yellow color - their mesotonal addition; and the pink - the multitonal addition and diatonic expansion of the ambitus based on octave equivalence. This figure is original.

This line of structural development is accompanied by semantic development, where "natural" modeling of sounds becomes replaced by "cultural" modeling. Timbral forms of music are based on onomatopoeic imitations of environmental sounds [12]. Ekmelic music adds yet another "natural" source - cross-modal (synesthetic) "linear" representation of native landscapes, wayfaring, and forms of locomotion by means of abstracting their characteristic features and imitating them by the shape of melodic contour [53]. Khasmatonal music enables representation of two or more "natural" prototypes in a single music work [383]. Oligotonal music marks the transition from "natural" to "cultural" models in melody-making, introducing the new level of abstraction of perceptual reality through the genesis of pitch-orientation. Thus, oligotonal modes are still used to represent animals by Khakass, Khanty, Nanai, Taz, Nivkhs, Yukaghirs, and Dolgans, supporting the onomatopoeic traditions, yet they also characterize the Nanai, Taz and Nivkh music for bowed pikolute that is semantically abstract and refers to the sphere of lamentations, funeral rites, underworld and female occupations [138]. Without knowing an appropriate convention, a listener cannot grasp the meaning of this traditional pikolute music. Sheikin and Alekseyev [136] underline that oligotony is the chronologically last of the schemes of tonal organization that maintains timbre as a primary expressive means: oligotonal pitch has inherently weak expressive capacities (due to its very few pitch classes), making timbre and rhythm the principal means of expression and identification of different tunes. In the next round of development, the inclusion of gaps between the mesotonal pitch-classes introduces melodic idiosyncrasy and supports modal creativity on part of music-makers, so that one song can contrast another in its tonal organization. This directs evolution toward multitonal modes, since introduction of gaps in melodies naturally leads to invention of the idea to fill up these gaps - generating extra pitch-classes (i.e., a gapped 3-degree mode promotes a stepwise 4-degree mode). Another typical method of creative expansion of a mode is what Sachs called "chain principle" [388]: upon becoming excited, a performer reproduces a salient motif above the upper margin of a melody, thereby generating an extra step or leap of the same intervallic value, which then becomes an extra pitch-class, once it is used repeatedly.

The succession of stages I-VI (Fig.6) can often be observed within a specific indigenous music culture by comparing songs collected by ethnographers, analyzing historic recordings (that date back to 1890s), reading the earliest ethnographic descriptions of music (dating back to the 18th century), tracking diachronic variants of the same songs, and comparing modern and ancient genres (e.g., Siberian indigenous folk songs about Lenin versus epic poetry). The Chukchi, Yakut, Nanai, Evenk, Nivkh, Orok, Khanty, Mansi, and Selkup cultures retain music created in schemes 1-10 [138]. A number of European cultures also retain traces of earlier stages of tonal organization, back to oligotony: Russia [389], Ukraine [380], Czechia and Slovakia [390], Hungary [391], 
Lithuania [392], Greece [393], and Azerbaijan [394]. All these cases support the model of progressive increase of complexity of tonal organization, expressed in the selection of pitch as the primary aspect of expression, increase of the number of pitch-classes, emergence of tonal hierarchies, abstraction of musical semiosis, and standardization of the incremental divisions for each of the principal aspects of music [143,383].

On the other hand, in the entire stock of literature on historic ethnomusicology, there is not a single account of a music culture that would lose its diatony and become oligotonal or timbre-oriented. The known cases of lost complexity are limited to specific genres or styles of music rather than the entire music culture (including all of its genres). As far as we know, music systems can become simpler only in regard to very complex forms of tonal organization downgraded to less complex, but not from complex to simple forms. Thus, the ancient Chinese music system passed through the reduction of heptatonic diatony that was established 8.6 kya to pentatonic diatony, adopted by the state, starting from the 2nd century BCE and eventually spread over all forms of music, including folk and entertainment [32,383,395-397]. In a similar vein, the ancient Greek music system lost its enharmonic genera, and its chromatic genera were entirely supplanted by much simpler diatonic genera after the 3rd century AD [143].

The divider between the capacity for regressive versus strictly progressive development seems to lie somewhere between the stages of multitonal modes and diatony. The "Rubicon" effect is probably determined by the discovery of standardization of interval-classes and possibly, incremental division of rhythm-classes and metric feet. Once a consensus of music-users embraces the standard of a melodic step or a paradigm of binary metro-rhythmic division, the music community cannot lose the obtained knowledge and the corresponding auditory skill in the same way as humans cannot forget how to walk in a bipedal manner. One standard can be replaced by another standard: e.g., a whole-tone step by a semitonal step, or vice versa - but not abandoned altogether in favor of non-incremental continuous (ekmelic) modulations of pitch. Ekmelic organization is still possible in an incrementally-oriented music culture but only as a peculiar device of expression, reserved for specific genres and styles, in other words, as an exception from a syntactic rule: e.g., glissandos or portamentos in Western classical music.

The principal agent that most probably holds each upgrade of simplicity in place is the social factor. Parallels between tonal organization of music and basic social structures have been reported by a number of ethnomusicologists. Lomax believed that song performance settings universally reflect social structures prevalent in a society: the practice of everyday person-to-person interaction directly influenced the manner of integration of singers in a vocal group [398]. He considered the organization of musical instruments in a traditional ensemble a symbolic representation of the formal organization of society. A clear demonstration of this was provided by Kubik: across many African cultures, families form regular ensembles of vocal and instrumental music, where instrumental parts are associated with ranges of human voices, categorized in terms of blood relations (father, mother, son, daughter, etc.), based on registral differences [399]. Social relations within a family become projected on families of musical instruments (xylophones, slit-drums, double-bells, and hand-drums) as well as the music that is performed on them. There are known traditions where family models are abstracted into a set of pitch-classes, each associated with a specific family member. Thus, Nsenga lamellophones represent boys with one row of tones, while girls - with another row [400]. Shona mbiras are even more sophisticated: the left lower manual represents "old men," the left upper manual_- "young men," and right manual-women [401]. The structures can be hierarchical - reflecting the inter- 
relations between mother, father, son, daughter, and grandmother [402]. Family musicking is exceedingly common in traditional societies across the world. Quite wide-spread is the practice of ensemble performance by the entire village (e.g., gamelan). Such practices are likely to promote the influence of the most common patterns of social organization on the typologies of arrangement of music. Friendly disposition towards all the participants in such ensembles finds a perfect match in the hedonistic nature of a musical mode: music-users develop conventional preferences for certain combinations of tone-classes (i.e., timbre-classes in timbral music, pitch-levels in ekmelic and khasmatonal music, and pitch-classes in pitch-oriented music), based on their perceived euphony. "Nice" combinations of tones might reflect on patterns of assembling groups of "nice" persons to produce these "nice" tones.

Yet another parallel can be drawn between subordination of pitch-classes in a musical mode and social subordination. The aspect of tonicity in music provides a convenient way to reflect the authority figure within a common social structure. Although the notion of "tonicity" was coined in Western music theory, it has been widely adopted to refer to the perceptual impression that a specific pitch-class in a pitch-set is more important than other classes due to its capacity to generate a sense of resolution of tension and provide finality at the end of musical phrases [403]. Experimental studies were able to identify the acoustic parameters that make a pitch-class appear "tonic": greater frequency of use, longer rhythmic values, greater amplitude, and salient position within a musical phrase and a melodic contour [404-406]. Tonicity plays an important role in tonality: musical keys are defined by identifying a pitch-class that features the strongest tonicity "tonic" - and adopting it as the "first degree". All other degrees are assigned the values of relative stability and instability in reference to the "tonic", forming a fixed system of hierarchical relations of subordination and coordination, by which a listener can recognize a specific key by ear while auditioning a music work [407]. "Stability" and "instability" here refer to the capacity of a pitchclass to, respectively, finalize a phrase or require its continuation.

However, in world's music, tonality is just one of many schemes of tonal organization - the majority of traditional music systems do not comply to the specifications of tonality and can be grouped under the umbrella of "modality," characterized by the absence of fixed hierarchies of tonicity [383]. However, they often mark one or more pitch-classes as more important than other pitch-classes. Hence, the description of their tonal organization requires a more sophisticated and context-sensitive approach than mere discrimination of "tonic" from "non-tonic." Following a distinguished music theorist of the early 20th century, Ernst Kurth, Larson proposed a useful model of "musical forces", where the force of tonal "gravity" enables stable tones to attract unstable tones, generating melodic motion - unstable tones gravitate toward stable tones until their melodic momentum runs out of energy and the melodic motion stops, thereby resolving tonal tension [408]. The advantage of this model is that, like Newtonian theory of gravity, it is capable of accounting for a variety of factors and supporting a number of possible schemes of "planetary" arrangement. From a musicological perspective, this model captures specifics of the analyzed melody much better than the rigid scheme of tonality that we know from textbooks on music theory. ${ }^{39}$

\footnotetext{
39 The inadequacy of Western theory of tonality to capture original traits of tonal organization has been demonstrated in numerous cases. Thus, Liszt was baffled over Glinka's March of Chernomor from his opera Ruslan and Liudmila unable to find the "tonic" of this music that otherwise fully satisfied the rules of Western harmony. The scheme of tonality has turned out to be overly "grammarian" - rigidly prescriptive rather than descriptive of the peculiarities of tonal organization that occur in practice of music composition.
} 
The evolution of tonicity constitutes an important part of the progressive development in complexity of music. "Tonal gravity" is a peculiar musical phenomenon, completely absent in speech and in timbral music. Schemes 1-3 (Fig.6) are characterized by the absence of pitch-classes and allow music to end at any point at the discretion of the performer. "Gravity" is formed throughout schemes 4-10 and materializes in the emergence of "tonic" at scheme 11 - this development was thoroughly studied by Alekseyev [136,155]. According to him, the term "tonic" is inapplicable to oligotony since the latter is characterized not by subordination, but by coordination of tones: in practice, melodic motion can stop on any tone, whether it is more or less melodically important, since there is no pitch-related tonal tension that would require resolution. Instead, melodic motion is charged primarily by rhythmic tension (shorter tones require longer tones to terminate a phrase). Monotony forges the "leaning tone," defined by the consistency and accuracy in hitting the same pitch level. Alekseyev qualifies such a function as "anchoring" (accidentally, this very term was proposed by Barucha within the psychoacoustic context in reference to a grouping mechanism that binds an unstable tone with a stable tone that follows it [409]). Ditony further emphasizes the leaning tone by assigning an "auxiliary tone" to support it. The latter can be distinguished by fluctuations in pitch values, shorter durations, and less frequent use in a melody. Tritony introduces a "contrasting tone" that challenges the "leaning tone" by posing an alternative anchor. Another common tritonic scheme is one anchor with 2 auxiliary tones. Both tritonic schemes become integrated in tetratony. The latter promotes the genesis of syntax and hierarchy into tetratonic modes, when music-users sooner or later discover the idea of pairing two auxiliary relations in one mode. This brings to life the antithesis of stable and unstable tones, where the former start specializing in terminating melodic motion, while the latter becomes its subordinate (while the other pair can be subordinated to this one).

All of this development easily finds matches in the line of social development. Monotony corresponds to the emergence of the sense of "self" in a child, ditony - to the mother-child relation, tritony - to the mother/father-child triangle, and tetratony - to the family unit that consists of mother, father, daughter and son. The further development runs in the direction of band-tribechiefdom-state. At its end, Larson's "musical gravity" obtains a socio-political correspondence: "tonic" (ruler) governs other pitch-classes, stable tones (ranks of aristocracy) "dictate" to unstable tones how to behave in a melody - each with varying powers of authority and levels of subordination - and unstable tones (commoners) "obey" their commands. Heterarchical/hierarchical relations of tonal coordination and subordination easily translate into socio-political structures. And this might be more than an analogy. As Blacking points out, the upbringing of children in pre-industrial societies is tied to the musical relationship between children and adults, used by the authority figures to reaffirm their authority and the subordinates to secure their safety [410]. Schemes of tonal organization might capture the specifics of agency, conflict, and cooperation in a particular society.

- The progression of monotony->ditony->tritony->tetratony->mesotony->multitony is as likely to regress as the ontogenetic progression of socio-emotional development: child$>$ mother/child->parents/child->parents/siblings, followed by further expansion of the family - all bonded by the mutual sense of attachment and care. The underlying reason is probably the ease of cumulative integration of simpler structures into more complex ones: the addition of new elements does not break the old connections, keeping them functional.

Kin relations have proved to be stronger than any political powers in Siberia as long as the traditional lifestyle was retained: no matter how the emerging states re-charted territories, clans remained the primary means of social organization there [245]. Citizens stayed loyal not to a state 
but to their clan. The weakest entity in the evolutionary progression of socio-political structures, which commonly disintegrates, is a state. Especially in antiquity, states as a rule were either conquered or ruined by revolts. As vulnerable were complex music systems, elaborated by states - subjected to radical transformations, including regressive ones, such as simplification by means of a political reform (the Ancient Chinese scenario) or decline of participation, leading to oblivion (the Ancient Greek scenario for chromatic music). Citizens of states are not nearly as conservative as clan members. After all, the ties of blood relation still tend to overpower the ties of citizenship, if in conflict, even in modern Western societies.

Yet another source that connects music structures to social structures is the musical parameter of voluminousness: musical modes are cultivated and acquire specialization for a certain number of participants - solo, duo, trio, etc.. Traditional music, designed for musicking-for-oneself and a partner, tends to not support advances in tonal organization. Even if one music-user accidentally comes up with introducing a pitch class in a conventional timbral musical mode, this advance is likely to be ignored by unrelated users and lost. What sustains an innovation here is its usefulness for satisfying a common need within a trusting circle of people, large enough to carry tradition. Thus, if a member of timbre-oriented music community overhears an unknown device of using a falsetto voice to represent a spirit from some visitor, that member might reproduce this device in representing his dialog with a spirit, where his normal voice (speaking for himself) would oppose falsetto (speaking for a spirit) within the same vocalization. This would be a step towards khasmatonal organization. If the entire community finds this invention useful, it might be developed into a khasmatonal mode, characteristic for some religious application. And at this point, the possibility of this mode regressing to a pre-khasmatonal scheme is little - as long as the corresponding rite survives. In contrast, the probability of khasmatonal organization upgrading to the ekmelic one is high, if, for example, the majority of music-users become attracted by the idea of smoothening the timbral contrast and filling the gap in a pitch contour by gliding (perhaps, to reflect the concordance with spirits).

The catalyst in the evolution of tonal development within a personal song culture seems to be the transition from family-based organization to multi-clan tribal and tribal confederations due to the population growth. The need to musically mark memberships in a clan and a tribe, to distinguish relatives from non-relatives, is the likely force that promotes the transformation of "monotonous" stereotypical oligotony into more expressive idiosyncratic mesotony. And once the means of generating contrasts within a musical mode are discovered, they become an object of creativity, promoting aesthetic attitude towards music and supporting social grouping and regrouping in a growing society.

The next milestone is the rise of the state that unifies multiple ethnic, linguistic, and religious groups, redrawing their affiliations according to systemic interactions within professional occupations, casts, markets, etc.. The process of restructuring society and establishing new social boundaries usually involves rationalized standardization of music intervals and families of modes, necessitating the emergence of formal music training and theory [143]. Once set, this "learned" (aka, "civilized") music culture is highly unlikely to revert to clan-based music even when a state is conquered by some militant nomads, as it happened during a century of Mongol rule over China, Persia, Armenia, and Georgia. Even a complete disintegration of a state, such as the Byzantine Empire, did not trigger the regress of its fully-fledged diatonic music system into simpler forms (e.g., mesotony) - nowhere across former Byzantine lands. 
One possible exception here might be the Inca empire. Rodolfo Holzmann made a convincing case for the surviving tritonic Q'eros music to represent the remnants of the Inca tradition [411,412]. This triadic music system is associated with the animal fertility cults, specifically the marking ceremony, and carnival celebrations in Southern Andes [413]. Tritony is more basic than pentatony and seems to constitute a trans-cultural phenomenon in the Andes [414]. Thus, tritonic triadic animal songs are cultivated in Potosí, Bolivia [415]. Tritonic system also survives in nostalgic bagualas songs and religious trumpet music in Northern Argentinian provinces Catamarca, Jujuy, La Rioja, Tucuman, and Salta [416]. The tritonic songs of the Atacama people of Northern Chile are believed to preserve a solid pre-Hispanic autochthonous tradition [417]. If Andean tritony indeed constitutes the Inca legacy, the Inca state would present a rare example of instituting the oligotonal music system - which would complement its highly unusual political and economic organization. However, even this case is unlikely to present a downgrade of tetratony or pentatony. More likely, Incas institutionalized a pre-existing folk tritonic tradition.

Here we should mention an intriguing observation by Fenk-Oczlon that the number of vowels and pitch-classes in a number of native languages and native music systems tend to match - most pronounced in the simplest and the most complex systems, but also observable in the stability of the average correspondence of 5-7 elements between all languages and musics [418]. Noteworthy, the official language of Incas, Quechua (that survived in Q'ero communities), uses a 3-vowel system. Another case of correspondence is between the tritonic chants of the Atacamenos in Chile and the extinct local language of Kunza. The Inuit culture presents the strongest modern case: Inuit language has 3 vowels, while Inuit music is based on the tritonic tradition - still retained in sparsely populated East Greenland and traceable in well-populated South East until a century ago [351]. On the other end of the spectrum, French and German languages feature one of the world's richest vocal systems (17 and 15 vowels), and their musical cultures are also characterized with the maximal number of pitch classes - 12 for well-tempered instruments (piano, organ) and 17 for nontempered instruments (string) and vocals. ${ }^{40}$ Evidently, French and German vocal systems have historically acquired extra vowels in parallel to their music systems, having evolved from diatonic to chromatic organization. This goes to illustrate that music complexity can be increased via iterated learning and cultural evolution, pretty much like language complexity can be increased through a cultural mechanism.

One could hypothesize that the common musilanguage platform bifurcated at some point into 3vowel proto-language and tritonic proto-music, following the paradigm "vowels sing but consonants speak" [420]. Their divergence probably accompanied the establishment of kinship, where music secured emotional communication between close relatives, and language started to support exchanges with referential information. Gradually, the dialogic musicking and solitary musicking-for-oneself became merged to form the collective musicking, extending the sphere of attachment and care to a wider circle of people living together. At this point, the opposition between "us" and "them" must have emerged, and the genesis of hospitality customs reconciled their dichotomy. Music must have been prominent in supporting communication with strangers

\footnotetext{
40 The most obvious demonstration of 12-tone tonal organization is the dodecaphony of the beginning of the 20 th century, developed in Germany, and its further development in serialism that found strong support in France after WWII. However, chromatic organization was prominent even within the traditional tonal music (e.g., Max Reger in Germany, César Franck in France). When chromatic music is performed on instruments other than keyboard and percussive and on vocals, it engages not 12 but 17 pitch classes, since all enharmonic tones are systematically tuned about a comma apart: e.g., $C$ sharp is higher than D flat, D sharp higher than $E$ flat, $F$ sharp higher than $G$ flat, $G$ sharp higher than $A$ flat, and A sharp higher than B flat [419]. This difference might not be obvious to lay-listeners but is observed by performers as part of "good intonation" - quite similar to the variants of basic phonemes of "o" or "a".
} 
who might have lacked the capacity to communicate with a host in a common tongue. Music has been reported as part of entertainment provided for the guest in hospitality customs.

Music also increased bonding within an entire tribe, facilitating the transmission, remodeling, and sophistication of know-hows via increased teaching/learning. Decreased reactive aggression is known to result in tighter and denser in-group networks, favoring prolonged face-to-face interactions and in-group knowledge sharing between adults, as well as between adults and children [421]. This presents enhanced learning opportunities for children (via prolonged exposure to a richer input and increased practicing) and improves teaching behaviors by caregivers (via the enrichment of the input and increased feedback). A prolonged juvenile period, which is typically observed in domestic animals as well as in humans, also is conducive to enhanced and prolonged play behavior. Play supports knowledge acquisition in many different ways: it increases exposure to the input, exercises learning mechanisms involved in the acquisition of abilities, enables the exploration of new constructions, and favors practice. This is how the feedback loop between prosocial behavior and music probably fueled the accumulation of knowledge and promoted cultural complexity, including complexity of music and language.

Both milestones in the evolution of tonal complexity, oligotony and diatony, as discussed above, have to do with the management of prosocial behavior and aggression. The transition from natural onomatopoeic models to cultural abstract models of encoding semantic information involves the substitution of timbre-orientation by pitch-orientation, which is closely related to expansion of musicking beyond the family circle and inclusion of listeners unrelated by blood. Timbral music is close-distance by design. Timbral musical instruments and vocals lose much of their meaningful detail over the distance beyond a few meters. Timbral transmission is severely limited due to the idiosyncrasy of timbral coloration and lack of objective criteria by means of which timbre could be quantized and reproduced. Timbre-oriented personal songs are by definition "personal" - they are peculiar to its original possessor since it is exceptionally difficult to reproduce a unique voice of a specific person. These traits confine timbral forms of music to a narrow family circle. On the other hand, conversion of personal song from timbral to pitch orientation opens doors to varied reproduction of ancestral songs or their elements by the offsprings.

Formation of the diatonic family of modes also involves profound social changes [143]. Most importantly, folk transmission, distinguished by active musical engagement of the entire population, reliance on audition and memorization skills (vigorous ear training), and absence of prescriptive formal music theory and the notion of mistake (creativity boost), becomes supplanted by professionalized music, produced by specially gifted and formally trained individuals according to a strict code of rational rules and fixed by means of some kind of notation [140]. This reorganization requires administration by some authority, political or ecclesiastic, and is limited to the territory controlled by those authorities across the ethnic, linguistic, and religious boundaries - involving all subjects of a state. Music becomes regulated and music-users develop a habit to discipline themselves in following prescriptive rules of performance and listening. Knowledge and veneration of such rules are viewed as the borderline between "civilized us" and "barbarian them". Importantly, the prosocial power of music is recognized only in regard to the "civilized us". Those seen as "barbarians" appear as a potential threat to civilization that justifies military control. Hence, the state-regulated diatonic music turns into a double-edged sword, friendly towards "us" and hostile towards "them". 
Similar development characterizes languages of such civilizations. Languages become normalized: standards of style appear, the grammar becomes codified and fixed, specific instructions about composition of speech are developed, the art of oration and art of literature emerge, leading to formation of new genres of oral and literal speech. Such changes become characteristic of the entire society of speakers of that language. Moreover, some languages become "scholar" (e.g., Sumerian, Sanskrit) and act as lingua franca, both synchronically, across the adjacent geographic region, and diachronically, across many generations (even in case living populations stop speaking such scholastic languages).

The earliest documented music theory - as retrieved from 29 cuneiform tablets from Ugarit, 14th century BCE - is written in the Babylonian cuneiform script (which is the historic variant of another ancient Mesopotamian lingua franca language, closely related to Sumerian - namely, Akkadian), supplied with music notation and lyrics in the Hurrian [422]. Just like the Babylonian music was regulated by hardbound rules for deriving pitch- and interval-classes and their application in specific music genres, Akkadian language developed quite sophisticated grammar and literature. Many Akkadian literary works manifest individual features of style and presence of "Mesopotamian self-consciousness," distinguished by the assumption that "foreigners, given the necessity and the opportunity, would adapt themselves to Mesopotamian culture" [423]. Akkadian was taught through formal schooling as an oral counterpart of the scripted Sumerian by means of drilling lengthy word lists, learning basic operations of measuring and book-keeping, and acquiring skills of composing documents and letters [424]. Noteworthy, a common form of teaching were the improvised dialogues between scribal students or a student and his teacher, master, or parent - teşîtu ('disputes') - which gave rise to the tradition of judicial rhetoric and where inventive invective was particularly appreciated [425]. Diatribe became one of the leading genres of the Accadian literature. Literacy was by no means exclusive to scribes: multiple texts were uncovered in domestic urban quarters, not authored by court scribes - evidently, simplicity and convenience of cuneiform scripting made it available to commoners for private correspondence and household administration [426]. Formal instruction by means of a grammatically developed language under administration of state authorities was bound to normalize that language and spread it along with the best products of a civilization that spoke it. This was how Babylonian sexagesimal division became cross-culturally adopted for measurements of degrees and time - just as Babylonian circle of the 5ths came to serve as the basis of diatony and tonality across many ancient states [427].

While "normalized" music and language consolidated societies of their users across ethnic and social boundaries, generally increasing in-group tolerance and prosociality, by the same token they promoted proactive aggression towards outsiders who did not share the same "norms". This tendency probably originated prior to the rise of first urban civilizations, most likely triggered by the Neolithic revolution, the resulting population growth (due to more efficient sustenance systems), and the need to protect one's property from neighboring hunter/gatherers. Since the beginning of the Holocene, humans have constantly formed relationships and alliances among non-kin groups for achieving collective tasks, including warfare [289,428,429]. As we noted before, such diplomacy stands in contrast with other species - even those that are also believed to have passed through a self-domestication process (bonobos). The latter do not engage in socially coordinated violence (incidentally, this is another reason why the term conspecific sympathy is preferable to the term self-domestication). In fact, one could hypothesize that exoteric languages might have facilitated the surge of premeditated aggression, as they are especially fit for conscious planning, thus potentially contributing to large-scale hostilities and escalated battles, and 
ultimately, to the emergence of cultural institutions around war and peace in complex societies [225].

Overall, it seems that the emergence of different types of music depends on the precise balance between the levels of reactive and proactive aggression. However, it is the "learned" music culture of first civilizations that adopted a permanent proactive aggressive stand towards outsiders. The entire course of development from the emergence of conspecific sympathy to cultivation of proactive aggression with regard to music evolution is laid out below.

\section{A model of music evolution}

Our model builds on the hypothesis that a phenotype similar to the phenotype observed in domesticated animals emerged gradually in our species as a consequence of a progressive decline of reactive aggression, triggered by diverse environmental factors. This allowed the development of increasingly sophisticated forms of music via the changes in our cultural niche, resulting from the behavioral (and perhaps, cognitive) modifications, brought about by increased social interactions. Although this process manifests itself most clearly in artifacts produced by late anatomically modern humans, we expect human music to have a longer evolutionary history within our clade, departing from animal calls. At the same time, because changes in aggression management can affect many distinctive human features, we expect our hypothesis to illuminate the common roots of music and language, their subsequent bifurcation, and potential reciprocal interactions, and more generally, the advent of modern behavior and culture.

Overall, our model encompasses 4 different stages, following musilanguage: proto-music, timbreoriented music, the genesis of pitch orientation, and tonality. It is beyond the scope of our work to discuss the putative musical abilities that the closest species to us might have exhibited, particularly, extinct hominins. However, because we see the evolution of music essentially as a continuum, we have subsumed this long history in the Stage-0 in our model. Stages 1-4 are hypothesized to be specific to our species, although we cannot exclude the possibility that other hominins, particularly Neanderthals, might have gone through stages of increasing complexity similar to Stage-1 and, perhaps, Stage-2 in our model.

\subsection{Stage 0: Musilanguage}

Within the hominin lineage, one can confidently expect that, at the beginning, communication did not essentially differ from animal calls. It might have relied on unintentional impulse-driven single signals, where an utterance reflected a certain affective state in reference to some object, action, or quality, observable to the signaler. This form of communication was called musilanguage by Brown [2] and, when used in group settings, probably engaged the isophonic texture - i.e., brief calls, continuously reproduced by multiple performers with random deviations in timing and pitch [207]. In his most recent update, Brown [227] postulated that cultivation of musilanguage, at first, generated the conventions for affective music-like prosody and, thereafter, formed the intonational prosody similar to language. We share his view.

It is difficult to assign specific stages in the evolutionary development of music (just as it is for language) to specific hominin species, but this starting stage could be associated with the first 
Homo species, perhaps from Homo habilis to Homo erectus. Homo erectus was the first Homo species to have left Africa - Dmanisi Man is securely dated to 1.8 million years ago [430], when the climate was not affected by strong cooling [431]. Plausibly, the migration was caused by the increase in population, in search of new territories. If that is the case, Homo erectus might have practiced collective vocalizations prior to its exodus from Africa and carried this tradition. Homo erectus hunted and gathered in groups, cared for injured and sick group members, while being violent towards non-group conspecifics (might have practiced cannibalism), was highly mobile, possibly used natural fire (at least episodically), possessed a minimal tool technology that barely exceeded the sophistication of tools used by apes and did not progress for about a million years [432]. The survival pressures, brought about by abrupt cooling that took place during the glacial maximum of the Mid-Pleistocene Transition, ca $700 \mathrm{kya}$ [433], seems to have forced Homo erectus to move to warmer areas in Southeast Asia, and reduced its population so that it did not survive past 108 kya in Java, Indonesia [298].

All in all, Homo erectus would be a suitable candidate for forging new forms of vocal communication, different from typical animal calls. These new forms likely included production of loud collective signals to scare off dangerous predators (delivered in the isophonic arrangement) as well as grunting patterns to accompany individual caretaking activities. Both applications are common amongst many mammals and both are related to the increase in friendly behaviors between the participants. At the same time, both applications contain traits that distinguish human musilanguage from animal communication. Animal groups, generally, do not use sounds in a premeditated coordinated manner. And the prolonged use of caretaking sounds had a chance of forging characteristic patterns, perhaps even forming a small set of specialized signals due to the greater duration of Homo's altriciality period, need for helpers during the delivery (because of larger cranial size of Homo fetuses), and the shortening of interbirth intervals that posed the need for childcare of multiple children [434]. The increase of overall duration, frequency of use, and the numerosity of caretaking participants likely stimulated the development of specific patterns of vocalization, more diverse and sophisticated than those used by primates.

\subsection{Stage 1: Proto-music}

At some point, musilanguage must have diverged into two forms of communication: emotional information (proto-music) and referential information (proto-language) necessary for collective execution of important activities (e.g., manufacturing of the essential tools and hunting). It is beyond the scope of this paper to delve into the distinctive features of proto-languages spoken (or signed) by other hominin species (but see [14,435-440] for reviews). As far as our own species is concerned, one could hypothesize that at this early stage, levels of reactive aggression were still high, thereby precluding prolonged contacts with conspecifics, long utterances, and cooperative turn-taking - instead, allowing just brief verbal contacts via short single-word commands, threats, and exclamations $[225,367,369]$. This is what we qualify as human proto-language (in distinction from hypothetical proto-languages of other hominins).

We can safely assume that some denotative words, aimed to describe the world around, were already available during this stage. Such are words referring to specifics of hunting, names for plants and other animals, and kinship terms [441,442]. The latter, in particular, show a notable phylogenetic stability across different languages, because of their frequent use [443]. The accumulation of a variety of referential words, perhaps, was one of the earliest signs of growing differentiation between proto-verbal and proto-musical styles of communication. The latter 
probably evolved through long-chain transmission of the stereotyped musilanguage signals, related to hunting and deterring dangerous predators, provision of individual care, and, possibly, as a safety signal. Each of such applications must have shaped its specialized forms for effective communication of corresponding affective states - in line with semantic specialization of animal calls but with greater diversity due to more complex behaviors. Hominin groups grew in size and social complexity, supporting the genesis of expressive means for conveying the most typical information, preparing the ground for the emergence of two proto-musical genres: aggressive "militant" (prey- and predator-oriented sounds, e.g., hunting and war chants) and loving caretaking (sounds of parental care, e.g., motherese and lullabies). Both types are described in detail by Jordania [205,444].

Genres are known to provide the framework for the development of individualized variants of stereotypical expressions. The emergence of the motherese might have been the result of accumulation of the variety of expressions within the rituals of childcare [436]. The specialization of labor and stratification of a group had capacity to also contribute to the emergence of new types of sounds, different from musilanguage as well as proto-language: e.g., the metrically entrained sounds of collective knapping could have been inadvertently discovered while making stone tools and assigned specific semantic values depending on the behavioral settings (ranging from the enthusiasm of anticipating the possession of a useful tool to the awe from participating in a mysterious ritual) [445]. Such collective "musical" knapping rituals were reported amongst the indigenous population of Queensland, Australia [446]. And archeological evidence for collective rock-knapping was uncovered in Neolithic sites at Sanganakallu-Kupgal, India [447]. It possibly existed earlier.

Yet another likely contributor to separation of language and music was the emergence of a new type of vocalizations based on hitting a pitch and holding it. Homo heidelbergensis that spread over Europe 700-500 kya was the first hominin that had anatomic capacity to sustain a certain pitch level [448]. ${ }^{41}$ This task requires a lower position of the larynx in the vocal tract, which must have emerged to support singing-like vocalizations as they obtained greater importance in social life [451]. Yet another confident proto-musical proxy is the anatomy of the inner ear and the audiometry of hominis species - the current research points to Homo heidelbergensis and Neanderthalis as sharers of basically the same hearing functionality as those found in modern humans [452-455]. Having the capacities to sing and coordinate the vocal apparatus with the heard sounds, early Homo might have accidentally discovered the effect of singing out, liked its sound, and used it repeatedly to experience pleasure - which would acoustically distinguish the protomusical application from proto-verbal.

\footnotetext{
${ }^{41}$ What increases the likelihood of Homo heidelbergensis coining proto-music and proto-language is its largest growth spurt of the prefrontal cortex amongst the entire hominin lineage [449]. Prefrontal cortex is responsible for such executive functions as inhibitory control and goal maintenance, crucial for cooperation, communication, and ability to stick to social norms in the face of conflicting situations. A number of traits in Homo heidelbergensis' lifestyle might have been responsible for this adaptation. Big-game hunting is dangerous and injury-prone; the chances of a kill for a solitary hunter are much lower than for a group of hunters; moving over long distances without help is hard for mothers, children, and injured individuals; taking care of multiple children by their mother alone is hardly possible in a nomadic lifestyle; and meat-sharing at the end of a hunt creates an incentive for everyone to participate in hunting since the exclusion of free-riders from sharing a prey is a universal trait of collective hunting (ibid.). Yet another collective activity, crucial for survival, that should be credited to Homo heidelbergensis is the controlled use of fire in hearths [450].
} 
The unprecedented instability of climate during the last half-million of years could explain the emergence of proto-music from musilanguage. Cool episodes promoted the advance in dwelling technologies, the controlled use of fire [450], and cooperation in hunting. Group cohesiveness and cooperation are known to increase species' ability to sur-vive extreme weather. Coordination of collective activities and teaching technologies to the younger generations required systems of communication and motivation far more sophisticated than those used in the animal kingdom. Also, depopulation during severe cooling was likely to instill closer relations between the tribe members and set favorable conditions for more intimate forms of bonding and dialogic musicking, similar to the interaction between a mother and her child. Subsequent warming, then, stimulated demographic growth and collective interaction. Then, fine-tuning of expressive means of protomusical communication previously established through personalization of relations and extensive use of solitary and dialogic vocalizations would pass through the chain of multiple transmissions. The latter had power to induce some kind of patterning in animal-like signals based on continuous modulations in frequency, amplitude, and time (see 3.1). The ongoing alternations of warm and cold periods were likely to forge and conserve forms of proto-musical communication that featured at least some conventional structural patterns of tonal organization within the most salient and easy-to-process aspects of expression (e.g., dynamic, timbral, and rhythmic patterns, as well as pitch contours).

Structural properties of proto-music might be reconstructed based on what we know about the emergence of the earliest forms of musicking in vocalizations of human infants during their first years of life. In a nutshell, infant's babbling diverges into "verbal" and "musical" types under the pragmatic pressures of effective communication with caretakers [29]. Namely, "verbal" type emerges from vocalizations intended to point at specific objects in the infant's surroundings and to demand specific things and actions. "Musical babbling", in contrast, emerges from instinctive vocalizations that entertain an infant and inform caretakers about the infant's state. Plausibly, Stone Age Homos mixed both, proto-verbal and proto-musical communication in an undifferentiated manner the same way modern human 1-year-olds interact with caretakers. This practice of proto-musicking might have been initiated by pre-human hominin species - perhaps, Homo heidelbergensis in Europe and other locations that were hit by ongoing oscillations of severe cooling and warming, causing contraction and expansion of social groups. It was more likely for proto-music to become different from musilanguage in harsher and more unstable climatic conditions of Europe, especially when cold. In order to keep fire burning during frost and to hunt big prey (a specialty of Homo heidelbergensis) group cooperation was a must. In this scenario, humans either learned proto-music from the northern populations of the Homo genus or, more likely, developed it themselves once they were out of Africa, closer to the cold regions. That said, we cannot discard the possibility that humans might have developed proto-music in Africa as a result of periods of ecological deterioration due to prolonged droughts.

Those few surviving genres of musicking of Arctic indigenous people that are based on sounds as byproducts of specific locomotive activities can suggest what proto-music might have sounded like:

Audio-5: Inuit assalalaa, children's game based on vocalizing on a single breath until exhaustion, while wiggling the entire body. http://chirb.it/wwEpbe

Audio-6: Chukchi pitcheinen dance "Girl-friends", rhythmic breathing vocalizations with falsetto sounds. http://chirb.it/bgC4P4 
Audio-7: Nganasan lullaby, rhythmic alternation between two pitch levels. This music might provide a glimpse into prehistoric lulling. $\quad$ http://chirb.it/PF7gKq

\subsection{Stage 2: Timbre-oriented music}

Timbre-oriented music is the best candidate for the first form of music to have emerged from protomusic. According to what we know about the use of timbral music in northeastern Eurasia, it retains many traits of animal calls: it often directly imitates the sounds made by animals, employs the same coding of auditory attributes for the emotional display (e.g., growling/hissing - for aggression, whimpering/whining - discontent, grunting/purring - pleasure, falsetto submissiveness), and is produced privately, "on impulse", rather than as a deliberate message to another party or for aesthetic appreciation by the audience.

As we already noted, frequent alternation of cooling/warming and/or arid/pluvial episodes almost certainly caused successions of population/depopulation, conducive to the emergence and spread of musical patterns. Timbral patterns were especially prone to benefit from the increased bonding during the cold episodes, because of the close range limitations of the transmission of timbreclasses. Timbre-oriented signals must have provided sonic markers to distinguish group members akin to how we recognize a familiar person by the sound of their voice. In contrast, episodes of demographic growth favored the spread of the established timbral structures across wider territory, preventing a timbral tradition from extinction.

What is most important, the population growth increases the number of song transmissions, both synchronically, between group members, and diachronically, between different generations. Each transmission tends to emphasize those traits of the communicated music that are shared by the majority of the community (see 3.2), so that the structural characteristics of music that are determined by social conventions tend to replace those characteristics that are determined by biological factors. This process must have made proto-music differ from music: the former based on the biologically ingrained semantic encoding (cross-modal correspondences, such as the use of growling timbres with little harmonicity for expression of aggression versus mellow timbres with high harmonicity for expression of submissiveness) as opposed to music based on conventions. The latter might violate the biological cross-modalities (e.g., the use of low register for the expression of love/care versus high register for anger/fear - see [13]).

A similar process of morpho-genesis has been identified for languages. At the earliest stages of evolution, formation of words is hypothesized to proceed on the basis of cross-modal iconicity (quasi-synesthetic connections between the auditory signifiers and the visual signified) - i.e., biologically driven association of vowels and consonants with certain objects and processes, present in speaker's environment and determined by similarity of their physical appearance to the acoustic properties (mostly timbral) of phonemes of the corresponding words [456]. As a language develops, the share of iconic ideophones and phonesthemes gradually diminishes, while the share of words based on purely referential (arbitrary) connection between certain combinations of phonemes and their lexical meaning increases, leaving just a bunch of onomatopoeic words. Overall, sound symbolism is untypical for conversational speech of the overwhelming majority of modern languages [457]. In contrast, symbolism bears a special importance for languages of animistic cultures, where speakers systemically employ ideophones which blurs the distinction between a speech event and a narrated event [458]. The same development characterizes the acquisition of languages in early childhood. If onomatopoeic words are usually the first to be 
learned, later vocabulary acquisitions show great reduction in sound-to-meaning iconic mapping [459].

The transition from biologically ingrained cross-modal matching of acoustic properties of sounds of music and language to learned cultural conventions relies on the broadening and deepening of friendly relations within a user-community. This is especially clear in the ontogenetic development. Infants start their acquisition of language and music only after establishing trust in relationships with their caretakers - i.e., learning to communicate precedes learning to speak and to sing $[56,90,345]$. Changes in loudness of the partials, relative roughness, sharpness and timbral width of child's vocalizations are implicitly adjusted to match the tonal qualities of mother's voice. In essence, intra-personal timbre-matching transpires into intra-personal sharing of semantic values (referential for language and affective for music). This binds the sound of words to their referential meaning and musical timbral classes to certain emotional states. Once such ties are established, both parties (parent and child) can revert the semiotic scheme: hearing a sound and experiencing a primed emotional state or evoking a denotation for a word (passive use) becomes as accessible as experiencing an emotion or wanting to refer to something and retrieving a suitable musical pattern or word from the repertory of known signals.

At this developmental point, the operation of matching becomes universal: person is matched to person, sign to meaning, meaning to sign, meaning to situation, and sign to sign. The latter probably found its origin in the articulatory base of the musilanguage - certain articulations were anatomically easier to combine than others. But once language and music diverged, language headed towards morphology (and more generally, towards increased combinatoriality and compositionality), whereas music - towards musical modes. We can expect the earliest forms of music to utilize "timbral modes" [12] in a manner prototyped by the infant's repertory of vocalizations during the first year of life. Thus, "abandonment cries" can be considered one musical timbral mode, as opposed to "happy cooing" as another timbral mode. Such specialization satisfies the musicological definition of musical mode as a model for abstracting a particular melodic style [460]: each type of infant's call is characterized by the choice of specific timbral markers, melodic contours, rhythms and dynamics, so that different infants as though all improvise variations of the same "crying" or "cooing" mode. The very first cries rely on the biologically ingrained vocal reflexes, but after some period of exploration, an infant usually elaborates more idiosyncratic patterns of expression.

What often confuses scholars is that timbral patterns of timbre-oriented music can be readily interpreted as "defective pitch" patterns by a person habituated to pitch-oriented music [268]. This misunderstanding is provoked by the presence of periodic spectral content in the euphonically timbre-matched sounds. The perceived harmonicity is often experienced as the fundamental frequency by listeners acculturated to pitch-oriented music and by possessors of absolute pitch. A universal developmental phenomenon amongst the children, raised in societies with pitch-oriented music, is that by the age of 3-4 they learn to process previously used timbral modes by reducing their spectrum analysis to frequency analysis and adopting the default strategy of looking for a fundamental frequency in musical sounds [29]. Yet another principal feature of verbal/musical divergence in infant's vocalizations is social: first verbal experiments occur primarily through the dialogic interaction with a caretaker, whereas first musical experiments (babbling) is solitary explored through playing with mouth in order to entertain oneself when alone (ibid.). This solitary hedonism, combined with the euphony of matching timbre-classes into a timbre-set (perceived as a ludic activity akin to construction games), makes the ontogenetic origin of music a fundamentally 
personal experience (solitary musicking based on personal liking of sounds) - contrary to the present consensus of scholars that zooms into collective functions that music acquired at the last stage of its evolution.

The transformation of proto-music into music must have followed the ontogenetic principles - after all, cultural evolution is directly formed through the ontogeny: cultural phenomena can exist in no other way but through the transfer from one generation to another, where a collective of people is always a sum of individuals. A society cannot sing a song. Only an individual can sing a song. And every generational transfer requires an adult expert catering to the psychophysiological limitations of an infant. Thereby, conservation of any cultural convention as a rule relies on the ontogeny.

A plausible scenario of the genesis of timbral music and its conversion to pitch could be as follows. Characteristic auditory patterns of grunting during grooming could have been reused by an individual during eating, based on the shared experience of pleasure - to generate a new characteristic pattern of humming. The use of the latter could further expand to apply to other relatively comfortable activities, such as food gathering or flint knapping. Emotional contagion and disposition to seek pleasure supported the possibility of adoption of such generalized humming as a signal of satisfaction by other group members. Then, the entire group would end up forming a semiotic convention in distinction from other groups. Due to significant anatomical differences in the length of the vocal folds between males and females, adults and children, the same pattern would be reproduced at different pitch levels. At some point, one member would notice this difference and deliberately imitate a differently-sized member, thereby abstracting the humming pattern that this other member originally produced. The reproduction of the same pattern at higher and lower registers side-by-side would make the change in a pitch level salient and thereby reveal the pitch aspect to everyone who witnessed such a reproduction, welcoming creative exploration of this novelty. Some present-day forms of timbre-oriented music give an idea of the way prehistoric timbral music might have exposed the pitch attributes.

Audio-8: Nganasan timbre-oriented personal song, created by parents for their daughter. It uses timbral markers, such as contrasting registers (growling and glottal), connected by gliding in pitch, vibrato and tremolo embellishments, while featuring pronounced changes in pitch levels. Obviously, reproduction of this personal song by its owner, a little girl, is bound to differ in pitch and timbre from this rendition by her father. http://chirb.it/7htOzK

Audio-9: Yakut romantic Jaw Harp duet. A female player keeps imitating the phrases of a male player until they join together in the conclusion. Although the music generally remains timbre-oriented, it contains frequency modulations and contrasts, more noticeable when both performers alternate. Substantial pitch and timbral differences are simply inevitable between two hand-made Jaw Harps. http://chirb.it/rH6bFD

Imitations of timbral music patterns by different people tend to bring out pitch-related differences, which are likely to become salient in chain transmission and attract attention of music-users, prompting attempts of their deliberate reproduction and exploration. The growth of population is particularly prone to intensify the use of pitch as an expressive medium. Since purely timbral qualities are hard to reproduce between individuals of different physique, the pitch continuum (higher-lower) turns out to be more instrumental for reproduction of timbral music than the timbral continuum (lighter-darker). This is especially true for personal song: the greater the demographic, the more important is the structural clarity in reproducing the "same" musical material (i.e., 
securing its identity). Perhaps, for this reason, personal songs of ethnicities whose music remains mostly timbre-oriented (e.g., Nenets, Nganasan) usually contain at least some tones that are welldefined in pitch (engaged as interpersonal markers, unlike timbre).

Geographic locations where climate kept changing the most, triggering demographic oscillations while remaining inhabitable, were the most conducive to the transformation of proto-music into music. According to reconstructions of the paleoclimate, Eurasia greatly exceeded other continents in this respect [461-463]. Therefore, the zone northwest and northeast of Levant (that was readily accessible for migration from Africa), was a plausible place to house the genesis of music. Yet another argument for Eurasian origin of music is the importance of kinship for the known timbreoriented and indefinite-pitch music systems, which probably come the closest to Paleolithic music (see 6.3). Evolution of Homo heidelbergensis into Neanderthals and Denisovans and their spread over Eurasia ca 500-50 kya resulted in interbreeding with early humans around the Altai region, testifying about the presence of conspecific sympathy (see 6.4). Differences in physique between the hybrid posterity and non-hybrid members of the same tribe might have helped distinguish one ancestral lineage from another. The byproduct of such lineage distinction would be the inevitable expansion of a circle of closely related individuals, accompanied by bonding with a greater number of people. This process is expected to have engaged the musical structures already established for the expression of love and care within the genre of motherese. The latter must have prototyped duetic communication between the pairs of closely related individuals. It is plausible that such vocalizations were somehow differentiated to enable the recognition of a specific relative following the model of vocal interaction between mother and child, where each is characterized by the contrasting acoustic patterns of expression. Such vocal communication could have received a proto-instrumental support: the sounds produced on simplest timbre-oriented musical "phonoinstruments" - common utilitarian objects used as knappers, scrapers, rattles, bull-roarers, buzzers, and shakers [464]. Their sounds are easily discoverable through common everyday activities and often inspire the favorite pastime activities of children, as well as adults in indigenous northern societies [465].

The time period ca 200-110 kya, corresponding to the long Riss Glaciation and the subsequent Riss-Würm Interglacial period, marked the gradual rise of symbolic behaviors in different parts of the world, evident in symbolic use of natural objects and manufacturing of decorative artifacts. The emergence of euphonic timbral modes that we regard as the borderline between proto-music and music should be seen as an embodiment of the same trend of appreciation of beauty and the first step towards abstraction and conventionalization of patterns of expression. Similar developments are to be expected in the domain of verbal communication. Deixis probably gave way to more abstracted use of words in the absence of the signified entities. Single-word utterances might have been combined in a pair-wise fashion - analogous to the combination of timbre-classes of music into timbral modes (and paired social relations). The generated compounds directed linguistic evolution away from onomatopoeic-based iconic semiosis toward convention-based symbolic references to objects and events. The increased prosociality and prolonged interpersonal contacts likely boosted the genesis of stable patterns of verbal expression, perhaps, putting in place the germs of grammar: the use of noun-like and verb-like words, morphological means for naming, distinction of commands and terms to refer to basic social bonds, but above all - derogatory expressions, thereby contributing to decline of physical confrontation [225,369]. The replacement of reactive aggression with verbal opened gates to intensive development of syntax by setting a feedback loop between the increases in verbal interaction and complexity of verbal expression. 
Finally, as reactive aggression started to decline as a result of a greater control of subcortical areas by the cortex, the compositionality of music increased. We can expect that other changes, important to both language and music, took place as well: the enhancement of our distinctive crossmodal thinking, that is, our ability to transcend the boundaries of core knowledge systems, which underlies our capacity for merging linguistic items and is also central to the figurative uses of language (e.g., metaphors, metonyms, and particularly, pragmatic inferencing) [368]. This enhancement of our cross-modality could account as well for the violation of the biological crossmodalities, both in the domains of music and language. ${ }^{42}$

\subsection{Stage 3: Pitch-oriented music}

The rise of pitch orientation in music most certainly followed the scenario similar to what we can observe today in the development of music skills during the first couple of years of life. All primordial vocal communication of an infant with caretakers occurs via the acoustic means that characterize timbre-oriented music: modulations in different aspects of timbre, such as darkness/brightness, thickness/thinness, registral coloration and harmonicity. Rhythmo-metric organization plays little role in distinguishing one type of infant call from another (apart from the general correspondence of pleasurable vocalizations to regular metric patterns, usually supported by locomotion - as opposed to metrically irregular calls expressing negative emotional states). Through ongoing dialogic communication with caretakers, an infant acquires the capacity to center on the pitch aspect alone and to continuously control the approximate intervallic size of changes in the pitch contour. This typically takes the form of gradual exploration and mastering of melodic intervals, starting from micro-intervallic steps to wider leaps and is closely related to the broadening of the vocal ambitus available for infant's vocalizations, which is caused by the growth of the vocal folds and lowering of the larynx [162,468-471]. The first distinction occurs between two general intervallic categories:

a) "Steps" - i.e., small-size, relatively smooth, incremental changes in pitch levels to a neighboring level, associated with the expression of "ordinary" intensities of positive and negative emotional states - versus

b) "Leaps" - i.e., large-size, abrupt switches between distant pitch levels, used to express "extraordinary" intensities of positive and, especially, negative emotional states.

Similar distinction plays a role in the prosody of verbal speech. The most likely explanation of the universality of this categorization has to do with the anatomical and neurophysiological properties of human hearing apparatus, determined by the Temporal Coherence Boundary [385] and the resulting segregation of audio streams [472].

Each of these two general categories becomes sub-categorized as a child grows - under the influence of the learned cultural conventions that determine the tonal organization of music observable to an infant. Thus, steps break into 3 types (see [383] for discussion):

a) "normative" - adopted as a reference and a default intervallic size; its exact size varies between different musical systems from about 100 cents for microchromatic traditions, such

\footnotetext{
42 Cross-modality is construed here (and in Section 8) in a wider sense than when applied to music (synesthesia-like perception patterns). We use this term to refer to our species' distinctive enhanced ability to transcend the limits of core cognitive systems (in the sense of Spelke $[466,467]$ ) and to draw relations between different domains. This ability helps us, among other things, (i) to merge concepts and form new ones, (ii) to chunk syntactic pieces and create more complex syntactic structures, and (iii) to metaphorize and metonymize, as in figurative uses of language.
} 
as falak in Pamir (see [386]) to about 200 cents for diatonic traditions that prevail in Western Europe (see [39]);

b) "small" - reduced in size in order to express an important characteristic nuance in an emotional state to distinguish it from another similar state; the size varies from about 25 to 50 cents [473] depending on the intervallic typology of a given music system; thus, each of "chromatic shades" in chromatic and enharmonic genera of Ancient Greek music was differentiated by the intervallic size of 17-33 cents (see [474]) and conveyed a specific semantic quality, ranging from "lugubrious" to "pleasant" (see [475]);

c) "large" - increased in size up to about 500 cents in very fast tempo in order to emphasize the contrasts between the adjacent pitch levels; such adjustment is common for expression of enthusiasm; the most obvious case of enlarging a step are the ditonic formulaic melodies with two pitch-classes that are about 200 cents apart at the start of a song but gradually expand as the performer becomes more excited (see [155]); pitch-classes of tritonic modes are often triadic and can be separated by steps up to 500 cents (see [136]).

Similarly, leaps break into 3 types:

a) "normative" - a reference size for a leap is determined mostly by the frequency of its use in a given musical culture, which usually is the smallest interval class that exceeds the Temporal Coherence boundary (i.e., about 300 cents for very slow music, 400-500 cents for moderate tempo, and 700 cents for fast music) - influenced also by aesthetic preference and specifics of a musical genre (e.g., plainsong is characterized by the minimization of leaps to project calmness; whereas military march, conversely, favors larger intervals, especially, the interval of a 4th, i.e., 500 cents, to project alertness and fitness);

b) "large" - slight exaggeration of the "normative" leap due to the need of expressing an increase in intensity of an emotional state (the genre of romance); typically, large leaps occur infrequently and exceed normative leaps by a normative step, usually forming a 6th (800900 cents), and, unlike normative leaps, require stepwise melodic preparation and termination (see [172]) - in contrast to normative leaps that can "zigzag" sequentially;

c) "extra-large" - strong exaggeration of the leap to express a rare outstanding burst of energy or an overwhelming emotional impulse, often negative, characteristic for genres specializing in expression of ecstatic or exalted states (lamentations), indigenous traditions related to evoking supernatural spirits, and/or singing in wide open landscapes, (e.g., Sami yoiks); the most common interval is octave (1,200 cents), less frequently, 7 th (1,000-1,1000 cents) and 9 th $(1,300-1,400$ cents), which are more common for pentatonic than heptatonic music systems (e.g., Mongolian aizam urtyn duu).

Each of these subcategories can be further subdivided. Thus, in Western classical music theory, 3rds are broken in major and minor varieties; 4ths - in perfect, diminished, or augmented; etc.. Such subdivisions take a long time to emerge, as evident in ontogenetic development - even musically trained children can reliably distinguish such intervallic inflections by ear no earlier than by the 8-9th year of life [476]. The pace for the cultural evolution of intervallic inflections is expected to be a lot slower, since humans did not have any external guidance, unlike modern children guided by adults. It seems that this second-order intervallic inflections were introduced during the late Bronze Age - the first documented intervallic system with varieties of 2nds, 3rd, 6ths, and 7ths was codified in Ancient Babylon (see [477]). The genesis of such inflections requires the adoption of an incremental standard for measuring intervals. 
The earliest pitch-oriented music most likely employed only the basic distinction between steps and leaps, as it is evident from the positioning of holes on the recovered Paleolithic bone pipes, aka "flutes" (see [395]).

In its earliest form, pitch-oriented music is likely to have emerged as part of a grand cultural leap 40-30 kya in caves of Southern Europe, the Balkans, or Levant. Its emergence can be seen as one of the consequences of the culmination of behavioral changes, resulting from long periods of extreme cooling, continuous depopulation and dispersion over wide territories, increase in cooperation, avoidance of incest, and more settled lifestyle in a reliable shelter (a cave with fire). Throughout the span of 40-17 kya, the metapopulation of Europe remained low, with barely noticeable growth rates (illustrated by general reduction of the number of known archeological sites compared to earlier times), lasting until $16.5 \mathrm{kya}$ [478]. By then, the growth rate tripled and the number of small sites nearly quintupled, signifying the intense dispersion of population by smaller groups (ibid.). Such demographic change provided the fertile ground for wider cultivation of personal music and development of pitch organization.

Paleoanthropological evidence indicates that reduced reactive aggression reached its peak by around $50 \mathrm{kya}$, while proactive aggression stayed at its minimum [290]. The beginning of this tendency can be dated by the beginning of the Würm Glaciation, ca 110 kya, when the first signs of behavioral modernity emerged in different geographic locations [479]. The peak of conspecific sympathy, ca $50 \mathrm{kya}$, was probably accompanied by greater frequency and diversity of intra-group contacts, leading to the emergence of the first hierarchical grammars expressing transitivity and the genesis of esoteric languages, similar to the ones spoken by present-day hunter-gatherer societies [225]. The same increase of cooperation and complexity must have affected music practices.

Life in caves played an important role in making pitch the most salient aspect of music. The cave echo prolongs a sustained pitch due to reverberation and resonance, thereby emphasizing the sustained phase in the ADSR envelope ${ }^{43}$ and directing the listener's attention to changes in pitch levels. At the same time, multiple reflections from walls smear and distort many timbral characteristics of a sound that otherwise are clear to the nearby listener - to the extent that the familiar timbre might become hard to recognize at a distance. Echo-caused transformation of timbre certainly contributed to music obtaining great importance in religious rituals, where a transfigured tone could be perceived as a voice of a spirit or a deity. Such attributions are reported in music cultures of those indigenous ethnicities that maintain traditional lifestyle: e.g., they use strange sounds in rituals related to magic [138]. Specifically, "singing caves" are worshiped in the Altai region, where natural sounds, such as wind, water, and echo are regarded as "voices" of "spirit-masters" [480]. A traditional Tuvan style of deep-throat singing, kozhagar kargyraa (Tuvan, "cave bubbling/rasping"), is characterized by unusually low, thick, and dense sound [181].

Audio-10. Tuvan kargyraa. This type of singing could have sounded in Altaian caves during prehistoric times. http://chirb.it/0N94qe

\footnotetext{
43 The Attack-Decay-Sustain-Release (ADSR) envelope is a standard method to describe a sound in electronic music, commonly implemented in envelope generators of synthesizers, samplers, and other electronic musical instruments. The ADSR model is very convenient for analyzing and comparing not only electronic but acoustically generated complex sounds that modulate over time/pitch domains - especially, to describe unusual timbres.
} 
Mysterious sounds coming from dark and labyrinth-like cavities, very different from habitual sounds of timbral music, must have prompted the association of pitch changes with supernatural forces and magic. It hardly can be a coincidence that the earliest sites of rock art in French Paleolithic caves, dedicated exclusively to depiction of animals (common objects of hunting, central to shamanic rituals), are usually located in those parts of the cave that bolster the strongest resonance [481]. Acoustic measurements confirm that the painted walls were indeed intended to reflect sound [482]. Many archeological megalithic sites exhibit a primary acoustic resonance at the average fundamental frequency of an adult male voice $-110 \mathrm{~Hz}$, suggesting that the caves were selected based on their acoustic properties [483]. Numerous Paleolithic caves in France, Spain, and Portugal contain painted stalactites that emit sounds of clear sustained pitch, once hit with a rock. Many such stalactites are chipped, indicating that they were indeed played, and their paint is visible only from a close distance [484], suggesting that the painted marks were intended for those who stood in close proximity and, perhaps, sang along the lithophone music. Dams inspected the French and Spanish caves with "singing rocks" and reported quite sophisticated scales: e.g., the pentatonic Nerja scale C-Eb-F-G-A-C [485]. The chance of their natural origin, without human "tuning," is small. Also, portable "musical rocks" might have been in use around these times [486]. At least starting from 36 kya, cave people in Southern Germany played bone pipes (aka "flutes") [487].

The reverberation in a large cave not only sustains pitch levels but also significantly increases the acoustic phenomenon of "tracing" in melodic intervals: for every pitch change, the decaying tail of the previous pitch overlaps with the attack of a new pitch. Practically, echo generates a momentary harmonic interval. And we know that discrimination of harmonic intervals relies on the inborn capacities of human auditory system [488], which is not limited to humans. A number of animal species can distinguish between different intervals [489], probably due to the crossspecific similarities of the neural paths involved in auditory perception [490]. Echo-driven harmonization of a melodic interval could explain the centrality of harmony and multi-part arrangements, as well as the discretization of pitch values, which are so pronounced in Western musical traditions. The task of collective singing while preserving the same melodic contour, in itself, was likely to promote discretization of pitch classes. Human ear is most sensitive to the acoustic parameter of roughness - i.e., vibrato-like oscillation in amplitude at a certain rate, perceived as acoustic "beating" due to the harmonic constituents of a tone coming in and out of phase by a certain period [491]. Roughness can make even a consonant harmonic interval (e.g., major 3rd) sound dirty or muddy when it is generated in the lowest registers (e.g., by two doublebasses). Maximal roughness is experienced when the frequency discrepancy between phases is 80165 cents, which is evaluated as unpleasant in most of the world's music cultures, except the few ones that adopt the opposite stand and regard it similar to the sound of a bell, their ideal of sonic beauty [45]. Chances are that the cave people, who did not know bells, evaluated semitone-sized harmonic mismatches between the singing partners as unpleasant and therefore tried to avoid them, which would effectively quantize their unisons and seconds. By the same token, they were likely to empirically discover the intervals that delivered the best harmonic fusion: perfect octaves, 4ths and 5ths.

Although Homo-inhabited caves are found all over the world, including China and South Africa, they became much more common in Europe and Western Asia, starting from about 100 kya in association with Neanderthals and early humans (Cro-Magnons) roughly until some 10 kya [492]. Living in a cave becomes relatively safe only when permanently guarded and the fire is always on to divert dangerous predators (cave bears). Although some scholars suggested that regular use of 
fire dated back to 1.6 million years ago in Africa, their claims have been contested [493]. Currently, the consensus leans toward the idea that habitual use of fire characterizes the colonization of regions outside of Africa, especially in Europe, where the temperature often stayed below the freezing point [494]. The European evidence suggests that early hominins migrated to Europe without the habitual use of fire and habituated its use only from about 300 kya [495]. Caves with fire must have provided shelter during the coldest episodes of the Last Glacial Maximum, promoting the cultivation of hospitality among cave dwellers - an ideal environment for the genesis of collective forms of music.

This scenario fits the available archeological data. A wide area from South Germany to Spain is covered by late Paleolithic caves, dated 36-30 kya, that contain the bone pipes (over 120 instruments recovered between 1995 and 2008, often 3 pipes per site), which are the earliest musical instruments capable of generating and sustaining multiple pitches [487]. The typological similarities in their construction [496], in light of the existence of a pan-European cultural network along the Danube Corridor throughout 45-30 kya [497], suggests a wide spread of pitch-oriented music. Importantly, all Paleolithic pipes uncovered so far have been located in Europe [484]. This exclusivity can be explained by the harshest cooling that severely cut populations (extinguishing the Neanderthals), promoting cooperation and hospitality, followed by the steady population growth, conducive to the ongoing accumulation of knowledge [498]. The growth started after the arrival of the first modern humans to Europe [499], reaching the rate of mean $0.1 \%$ per year [500]. From the onset of the Neolithic, the abundance of easily accessible valleys with persistently mild climate turned Europe into the arena of rapid development of technologies and arts. In relation to music, this trend transpired in the invention of different musical instruments, genesis of a variety of instrumental performance techniques, interaction of vocal performance with instrumental, and the emergence of rules that regulated new collective applications of music.

Generation of pitch-oriented music in Europe should be considered one of the markers of cognitive modernity - in contrast to timbre-oriented music that has retained its stronghold at the opposite end of Eurasia, from the Urals to the Russian Far East and Alaska/Canada. The role that Paleolithic "bone flutes" played in formation of pitch- and interval-classes might be analogous to the role that Jaw Harp played in eastern Eurasia in conserving the tradition of timbre-oriented musicking [12]. The earliest uncovered Jaw Harp dates back to 4-3 kya, in China, but dispensable Jaw Harps made of grass and tree twigs, found amongst the indigenous populations of the Russian Far East, might have been used much earlier - absent from archeological records due to their rapid decomposition [501]. The archeological evidence is conclusive that Jaw Harp was brought to Europe after the 13th century AD from Asia [502].

Jaw Harp tradition is based on the unique system of vowel articulations that differs from local languages, yet seems to have influenced linguistic vocal systems, specifically, vowel harmony that characterizes many indigenous languages of northeastern Eurasia, clustered and enclosed in a huge geographic area between Japan and Turkey (see the map in Fig.6 in [501]). Noteworthy, vowel harmony in languages of the Mongolic and, especially, Turkic families exhibits essentially musical traits that make the native speakers sound singing-like. The "musical" features include: matching specific vowels within a word, regularizing the distribution of vowels and consonants, fixing the position of the accent in a word, and stabilizing the short/long opposition of vowels - which generates a binary metric framework. Turkic languages adopt a specific "harmonic quantor," systemically employed to quantize the timbral qualities of speech into "classes" of harmonic relations [503], while superimposing the metric regularity that induces rhythmic relations between 
syllables [504]. In essence, such organization combines timbral modes of the same sort as timbreoriented music with rhythmic modes, cultivated in the same geographic area [501]. Virtually all native words receive such treatment. Moreover, vowel harmony also regulates pitch levels, since back vowels tend to sound higher in pitch than front vowels, while labial vowels - lower than nonlabial (ibid.). Such structural similarities between languages and music autochthonous to the Altai region suggest their very ancient mutual roots in musilanguage.

Pitch-oriented Western Eurasian music, well-differentiated from languages, generally opposes timbre-oriented Eastern Eurasian music that retains many common traits with local languages. This distinction can be characterized by yet another set of general cultural traits, noticed by humanitarian scholars (see e.g., [505-509]):

- "Progressive" and active (constructive) psychological culture of the West. Westerners tend to view the present as "in the process of making for the future" and focus on the development of technological means to restructure nature to satisfy the needs of humans. This is a clearly social and collective task that relies on cooperation and organization, as well as accumulation of knowledge;

- "Regressive" and passive (contemplative) psychological culture of the East. Easterners tend to see progress as something potentially detrimental to valuable and venerated traditions of the past, and therefore, adopt the strategy of curbing one's demands in order to minimize human interference in what appears as "natural" order of things. This is a clearly psychological, and therefore individual, task that relies on voluntary control of emotions and thoughts, as well as preservation of knowledge.

This contrast in cognitive methodology and culture of thinking is by no means hard-cut, but is most pronounced in the philosophy of arts and music - leaving a noticeable imprint on history of arts (e.g., [510-512]. It is hard to miss an obvious correspondence between the constructivist Western cognitive model and the collective nature of pitch-oriented Western music, on the one hand, versus the conservationist Eastern cognitive model and the personal nature of timbreoriented music, cultivated exclusively in northeastern Eurasia, on the other hand. Their antithesis is embodied already on the elementary level of tonal organization of music.

- Timbre-classes can be discriminated only at close distance, when a sound generator operates alone, unmasked by other auditory information - quite similar to the pragmatics of speech, determined by the intelligibility of phonemes. Therefore, timbre-oriented music usually takes the form of a self-communicated monologue (the babbling model) or an intimate close-up dialogue (the motherese model), where both parties can clearly capture and interpret timbral nuances.

- Pitch-classes can be discriminated at long distances, even a few miles away [13], and benefit from the "chorus" effect produced by multiple sound generators [12]. Switches between different pitch levels become emphasized and therefore more intelligible, when generated in sync by vocal or orchestral tutti. Therefore, pitch-oriented music supports collective performance up to enormous sizes (thousands of participants). ${ }^{44}$

\footnotetext{
44 A number of symphonies at the turn of the 19-20th centuries were scored for extremely large settings (Mahler's Symphony No.8 was even nicknamed "Symphony of a Thousand). Older classical music was also performed in largescale settings. Thus, Handel's oratorio "Messiah" has been performed by up to a thousand participants for over 200 years in commemorative concerts in England - documented in a Beulah CD (\#1PD1) "Live at the Crystal Palace: Choirs and bands recorded 1926-1932" (1993). Gigantic recitals were common in the US throughout the 19th century. For
} 
This distinction reveals the fundamental difference between timbre- and pitch-oriented music systems: pitch orientation supports and promotes the growth of a performing group, whereas timbre orientation, in contrast, prevents such growth and effectively conserves musical tradition. Inclusion of more performers necessarily causes modification of musical structures, since each participant inevitably introduces slight deviations from the model known to all participants, even when all are trying to accurately reproduce the very same melody performed in unison - so-called heterophony [207]. Multi-part textures introduce even greater contrasts of parts. Oral transmittance of the same pitch-oriented music also causes substantial changes, creating variants and "offsprings" of a song, often leading to the genesis of new songs, like an ancestry tree that can be analyzed following the genetic approach [274]. The more well-defined in pitch and complex the song, the more it is susceptible to variation. Thus, folk multitonal songs are characterized by high transformation rate, pronounced even over 2-3 generations (see 6.5).

Traditions of timbre-oriented music are characterized by strong conservatism due to their extensive solitary and personal use. Songs "for-oneself", wide-spread throughout northeastern Eurasia, retain their arsenal of expressive means virtually intact over generations [264]. Alekseyev, one of the experts on such music, explains this by the absence of the rhetoric factor: self-communication does not require to convince anyone in the vitality of a communicated emotional state - unlike songs "for-others" that are judged by the other party and therefore must effectively project a specific musical emotion [136]. The need to convince usually motivates a music-maker to seek ways to amplify the desired effect, causing the revision and the update of expressive means (melodic intonations, rhythms, etc.). As a result, emmelic songs that are frequently performed "forothers" cause musical tradition to quickly metamorphosize. The growth of the audiences transpires into the greater contribution of the rhetoric principles to the evolution of music idioms. In addition, performers tend to become more competitive in seeking public appreciation, which triggers the "race" for greater expressivity and technical execution. Once started, such trends usually do not disappear and lead to professionalization of musical occupation and the institution of authorship [514]. That is why even the briefest books on the history of Western music are so voluminous and increasingly so in their coverage of the more recent times, when the pace of innovation and complexity substantially increases [515].

In contrast, dialogic forms of timbre-oriented music are as conservative as "songs-for-oneself". For instance, motherese demonstrates an amazing stereotypicality across various cultures and languages [516]. The reason for this is that ritualization of timbre-based genres relies on biologically ingrained psychoacoustic functionality of expressive patterns of timbre. Thus, motherese ritualizes mother-infant interaction in a way that resembles animal rituals, described by ethologists and known for their stability. Motherese shares many patterns of encoding with animal communication [517]. Auditory, visual, and locomotive cues in parent-child interaction are coordinated based on instinctive impulses rather than learned conventions [518]. Such rituals prove to be much more resilient to change than many forms of pitch-based music.

Yet another important distinction is that pitch domain inherently involves "pitch correction" in collective monophonic performance: the majority of singers with normal singing abilities automatically match their voices in order to achieve the closest possible match to their singing

instance, Patrick Gilmore's concert for the World Peace Jubilee of 1872 engaged 20,000 instrumental and vocal performers [513]. The earliest record of the gigantic performance is the grand procession at Alexandria, held by Ptolemy II in the 3rd century BCE, which involved 600 singers and 30 citharists [372]. 
partners in rendition of the same melody. This "tuning reflex" seems to comprise a biological universal for humans, shared with at least some animals, such as primates and cetaceans [519]. The FMRI study of human singing demonstrates that involuntary pitch-matching response occurs when the frequency mismatch is 25 cents, replaced with voluntary response when the mismatch is 200 cents [520]. Intuitively, singers try to sing known melodies in unison/octave, or, if their singing ranges do not allow for this, in other "perfect" intervals: 5th or 4th [521]. What this means for pitch-oriented music is that formation of intervallic subclasses to a large extent is factored by the cultural preferences of the majority of music-makers within a music-user community.

In collective monophony, pitch values observed by the majority of participants become the tuning standard for everyone. This is how specific scales are born and why they often bear "collective" ethnic or geographic names (e.g., Gypsy, Istrian, Hebraic, etc.). Deviation from such a standard is perceived as a mistake. This situation is very different from timbre-oriented music that knows neither rigid norms for production and reproduction of timbre-classes, nor notions of mistake. In indigenous music, timbre tends to remain idiosyncratic: music-users recognize singers and Jaw Harp players by their unique tone and individualized patterns - not that different from how we recognize a familiar person by the sound of that person's voice and patterns of speech [12]. The uniformity of tuning to a certain pitch standard in the genesis of emmelic music must have come from the harmonic uniformity of the pitch parameter of various instrumental sources, such as "music rocks", whistles, or bone flutes, used along with singing and imitated by singers $[39,136,138]$. The referential authority of pitch in such settings most certainly relied on supernatural justification: a pitch generated by a stalactite would have been interpreted as the voice of a cave spirit, a pitch produced on a flute made of bird bone - as the voice of that bird's totem, etc.. Such attributions are exceedingly common amongst Siberian indigenous ethnicities that retain nomadic lifestyles and practice timbre-oriented music [138].

Pitch correction occurs not only during collective music-making but through successive chains of transmission from person to person that tend to discretize, quantize, and diatonize the initial models [139]. Neural constraints and biases for each of participants in cultural transmission impose structural modifications on musical structures: the smaller the information-processing bottleneck, the larger the pressures to regularize the music material to facilitate its processing in the brain [522]. The cumulative result of a long transmission chain is that a model-melody based on indefinite pitch (i.e., ekmelic intervallic typology) is likely to acquire definite pitch (emmelic intervallic typology). Thus, pitch reference, once set in a sizable community, is likely to override timbre-orientation. This conclusion stands in agreement with the developmental ontogenetic theory of "musical hearing" naturally growing from "verbal hearing," as formulated by Teplov [523] and experimentally confirmed by Leontyev [524].

The most important consequence of switching from timbre- to pitch-orientation is the unleashing of progressive growth in the number of participants in a music act, supported by a music system: from an individual alone to a pair of relatives, then, the basic family, the extended family, the entire clan, the tribe, the tribal coalition, the confederation of tribes, a city-state, nation-state, empire, and federation of states. Throughout this progression, music becomes the tool for sociopolitical unification of an ever-growing body of people to coordinate their joined cultural activities and achieve results greater than possible for a smaller body. The entrainment properties of music and its capacity for emotional contagion secure consolidation of collective interests, attitudes, and motivations to the extent that the entire group starts functioning like a single super-organism. This creates an incentive for group competition of the exponentially growing scale, by far exceeding 
anything known in the animal kingdom. Pitch-oriented music fuels the competition between families, kins, tribes, tribal coalitions, and states.

Emmelic music becomes a double-edge sword: it increases the bonding inside of a group while alienating its outsiders. Such alienation is not congruent to aggression - normally, insiders remain relatively well-disposed towards strangers. However, it is quite common for any social group to develop animosity towards at least one or two outsider-groups, once they are perceived as a potential threat. Regulation of this propensity becomes the prime goal of Stage-4. But before proceeding to its description, we have to introduce a very important notion of intonational typology, without which the qualitative leap between Stages 0-3, on the one hand, and Stage-4, on the other hand, is hard to understand. It is the antithetic relation between the 4th intonational type and 3 earlier types, easily combinable together, which must account for the peculiar combination of minimized reactive and maximized proactive aggressions that characterize Stage-4. Two evolutionary earliest types of intonations are also of interest to linguists, since the phonology of human proto-language was not yet differentiated from intonations of proto-music.

\subsection{Intonational typology of music: from personal to collective}

The transition from timbre-oriented personal to pitch-oriented collective forms of music involves not only conversion of timbre-classes into pitch-classes, followed by the quantitative increase of the latter. The elementary unit of constructing a music composition is not a tone-class but a musical intonation. A tone-class is akin a phoneme of language - it does not carry any semantic value and works like an atom to comprise the material to build structures of music. The choice of material does influence the functionality of music, but in the most general way - e.g., timbre-classes make music private, pitch-classes - collective, rhythm-classes - energetic (impulsive and unpredictable), meter-classes - inertial (patterned and predictable), etc.. However, the primary elementary unit that makes music meaningful (akin to a word in language) is a musical intonation. It is used to build a melody - i.e., the most expressive aspect of music and the most common embodiment of a musical theme. As Teplov thoroughly explains [523], intonation "glues" musical structure to experience, opening doors to semantic interpretation, and mediating between memory and attention: the listener decodes melody by recognizing familiar intonations, while identifying and memorizing new ones. It is the convention of musical communication in a given culture that prompts the listener to connect certain tones, acknowledging the meaningfulness of their relation, as opposed to breaking other tones apart as belonging to different intonations. This intuitive competence plays a key role in experiencing the melodic interval - an interpretative effort of matching the detected change in frequency to the known glossary of meaningful melodic intonations and choosing the closest match based on the context of a melody.

Music-users intuitively build their glossaries of musical intonations peculiar to a given cultural context. Each cultural formation can be characterized by an assortment of specific intonations [378]. And frequency of distribution of these intonations shapes a musical mode [6]. The ultimate selection of pitch-classes for a composition is determined by a set of intonations most relevant to the intended expression. A creator of music conceives music by deciding not which mode to use, but which musical genres and styles are the closest to what he has in mind - then, retrieving the suitable intonations [17]. A recent statistical database study of pools of melodies supports Asafyev's claim that certain styles of music can be defined by their intonation prevalence [525]. Typology of content leads to typology of form - a musical mode becomes a container for storing a set of intonations common for expressing a certain topic, thereby acquiring semantic value and 
formative power for music-making (e.g., major mode makes music happy, while minor mode sad) [526].

Private forms of communication through music can evolve into collective forms only when musical intonations pass through radical transformation. The very first step towards the genesis of pitch orientation (quite obviously) must be the adoption of some kind of a reference pitch-level for relating timbre-classes along the axis of pitch. Russian ethnomusicologists qualify such organization as ekmelic [383]. ${ }^{45}$ It is based on ekmelic (indefinite) intonations, embedded in ekmelic modes. Systemic use of indefinite pitch makes the standard Western term "pitch class" poorly suited to the description of ekmelic modes, even when they appear to sound pitch-oriented rather than timbre-oriented. Only the reference pitch - ekmelic "anchor" - can be conceptualized as a genuine "pitch class". The rest of the mode has to be distinguished as "degrees" (i.e., coordinated pitch levels), following the paradigm of degrees in a musical scale, since the registral order is cardinal for ekmelic modes. Tones of ekmelic music can be classified only in relation to their registral position towards the anchor (higher or lower) and rough qualitative distinctions between steps and leaps (small vs. large). An analyst can distinguish one unstable degree from another by its timbral coloration, registral position, melodic function (complementing or opposing the anchor), and its phrasal position (opening, closing, climax). The intervallic distances between the degrees often fluctuate in size $(<\mathrm{a} 3 \mathrm{rd})$ between the repetitions of the same verse or between different performances of the same song.

Audio-11: Sae Dyige-dyige, Yakut lyric song. Two performances of the same song (consecutive) by the same singer, where the pitch values of degrees shift between both versions, causing the expansion of the ambitus (the same singer sung Russian songs without such a shift). Courtesy of Alekseyev, used by permission. http://chirb.it/g36sC2

Typically, an ekmelic musical mode is restrained to a single vocal register and contains an anchoring degree that varies in pitch value much less than 2-3 other degrees (anchor is "stable" in a sense of greater stability in tuning - vs. "unstable", i.e., volatile degrees). The most common unstable degree is complementary - usually 100-500 cents higher or lower than the anchor, shorter in rhythmic values, and less frequent in the melody. Complementary melodic motion generates easy-going waiving. Less common is the opposing degree, positioned further apart from the anchor and challenging the latter by featuring nearly equal rhythmic values and frequency of use. Alternations of this degree and the anchor sound more aggressive than the auxiliary interaction of the anchor and the complementing degree. The least common is the extreme degree, placed at the maximal distance, near the margin of a vocal register, and acting as a melodic "dissonance" by disrupting the melodic continuity (the other 2 melodic functions are "consonant" - i.e., they support the melodic continuity). ${ }^{46}$ Unstable tones vary a lot in their frequency values for each of their

\footnotetext{
${ }^{45}$ Western ethnomusicologists qualify this as "smooth" pitch (in contrast to "striated" pitch) [527].

46 The term "consonant" here has nothing to do with linguistic consonants. The antithesis of "consonant" and "dissonant" here follows the tradition of opposing the harmonic intervals, whose tones have simple frequency ratios and fuse well, to those intervals, whose tones have complex ratios and do not fuse well $[44,46,490]$. This relationship of concordance vs. disturbance of the contemporaneous combination of tones (harmonic) finds an analog in concordance vs. discordance of the successive combination of tones (melodic). The latter, however, features a very different taxonomy: e.g., the most consonant melodic interval-class - a 2nd (aka "step") - is a dissonant harmonic interval-class (see [383] for the discussion). A note of caution: these distinctions refer to the objective psychoacoustic properties of combinations of tones rather than the aesthetic categories of pleasure and displeasure (which can agree or disagree with the psychoacoustic properties in different musical cultures). Although ekmelic melodic intervals are indefinite, their perception, nevertheless, is governed by the same Temporal Coherence Boundary [385] as the perception of definite intervals.
} 
occurrences in a song and often glide in pitch (very much like pitch levels of vowels in verbal speech).

Audio-12: Nganasan table song. An example of the 4-degree ekmelic mode and ekmelic intonations. http://chirb.it/L7O31C

Ekmelic mode belongs entirely to the domain of personal music. Collective performance would inevitably render unstable degrees unrecognizable: each participant would alter the pitch value of unstable degrees differently, depending on one's emotional state (the greater excitement, the wider intervallic fluctuations), creating irregular irrational clusters. Hardly the resulting clusters of pitches can exert the unifying power over the participants like joined singing of a pitch-based song. The bonding effect in rare collective forms of ekmelic music, if present, comes not from melodic intonations but from extra-musical factors, such as collective synchronous motion or the awareness of a participation in a ritual:

Audio-13: Even sedye, Indigirka region "responsorial" song-dance of totemic nature. This collective genre, exceptional for indigenous Siberia, features stochastic clustering of tones of different pitch values between all the performers. It is mainly the commonality of the dancesteps in a round-dance formation (and the resulting common rhythm) that generates the sense of unity between the participants. http://chirb.it/GEDEqq

However, adding a musical instrument, such as a "singing rock", a whistle or a bone flute, to the performance of an ekmelic song radically changes the format, triggering the process of instinctive pitch-matching. Delegating the function of an anchor to an instrumental timbre that drastically differs from a human voice provides an excellent pitch reference.

Audio-14: Selkup song "Over the lake", performed with the accompaniment of a spoon. The accompanied sections contrast the indefinite solo singing by matching the pitch level of the stricken spoon. http://chirb.it/LsALmp

The importance of instrumental reference for indigenous Siberian music was noticed by Yurii Sheikin, the leading expert on the history of Siberian music [138]. He distinguished between two types of musical intonation: vocal "psychophysiological" - aka, "natural" in a sense of adapting the capacities of a human voice to the task of reproducing the sounds of natural sources (animal calls, wind, water, etc.) - versus what he called "organophonic" intonation - characteristic for a musical instrument or a phono-instrument (spoons, saw). Indigenous people usually perceive instrumental sounds as "voices" of various material or spiritual objects (following animistic beliefs). Once discovered and adopted for cultivation, organophonic intonations penetrate into the domain of vocal music and start influencing psychophysiological intonations. Their interaction occurs in the genres of accompanied solo song. Thus, most indigenous peoples of Western Siberia possess at least one such a genre and hold it as a backbone for their musical tradition [138]. Music users habituate to the sound of such a "backbone genre", so that important vocal and instrumental intonations both become fused into a single musical mode that serves as a model for future attempts to musick within the corresponding genre.

Audio-15: Altaian epic tale "Kan Altyn" with the accompaniment of tovshuur (lute). Instead of using a timbre-oriented model of solo deep throat singing, common in Altai, the singer uses the pitch-classes supported by tovshuur. http://chirb.it/1w6xrz 
The role of the feedback loop between vocal and instrumental music in the evolution of tonal organization has been barely researched, pioneered by such Russian scholars as Beliayev [39], Kushnaryov [528], Alekseyev [136], and Sheikin [138]. To sum up their findings, the transition from stage- 0 to stage- 4 of our model can be seen as 4 shifts between different types of musical intonations, where "intonation" is understood as a preferred method of sound generation, articulation and resonance while connecting two sounds together in a single motif - what Kvitka called "ethnophony" [380]. These 4 types are not mutually exclusive. In some cultures, one type supplants another type, whereas in others they coexist, isolated from newer types by being conserved into their proprietary genre niche [136]. Below we describe each of these types and their typical performance format.

1. Anthropophonic intonation was defined by Alekseyev [529]. It is generated by vocal articulations similar to linguistic phonemes, where different parts of the vocal apparatus are engaged to modulate pitch levels (e.g., glottal, nasal, wheezing, croaking, or subglottal utterances within a selected frequency range). The resultant melodic intonation is not really "pure pitch," which is the product of "true vocal folds," but "dirty pitch," modulated by "false vocal folds" [530]. Such intonation might appear to possess a pitch value or to follow a specific melodic shape, but in reality these outcomes are unintentional byproducts of the production of timbre-classes in a way that is essentially similar to constructing syllables by combining the vowel phonemes (pitch component) with the consonant phonemes (noise component).

Anthropophonic intonation is a principal building material for what we identify as stages 0 and 1 . It is determined by "natural" anatomic factors, such as respiration rhythms, heartbeat, patterns of locomotion, and expression of vocal registers. It can be used to produce "personal" vocalizations (e.g., during grooming) as well as isophonic "collective" vocalizations (during hunting). In the latter case it generates undifferentiated loud noise that satisfies some utilitarian need rather than aesthetic appreciation. Although this intonation requires one sound producer at a time to support musical communication, it can be used "dialogically," in a format of a musical responsorial. This intonation is not combinatorial: one type of intonation cannot be combined with another to form a compound whose expression would combine the expression of both types. However, an anthropophonic intonation can be repeated like linguistic reduplication, possibly numerous times, involving timbral variations. Anthropophonic intonation is usually made of 1-3 timbre-classes, joined together due to the anatomic complementarity of their articulation and commonality of their expressions.

Audio-16: Even throat song. Timbre-oriented music. This song, performed by a woman, does not even sound like a female voice. It is based on the deep throat phonation in combination with the breathing rhythms. Its timbral mode consists of 3 timbre-classes, engaged in 2 intonations that keep alternating. http://chirb.it/vq5H3w

2. Naturophonic intonation - this is how we prefer to rename Sheikin's "psychophysiological intonation" [138] for the sake of terminological consistency based on specifying the tonal source for an intonational type. The term "naturophonic" highlights the propensity to imitate environmental sounds by vocal or instrumental means - using basic musical instruments (e.g., scrapers) and phono-instruments (decoys). Naturophonic and anthropophonic intonations share timbral orientation and lack of pitch coordination or rhythm syncing between multiple sound producers, when engaged in simultaneous musicking (Audio-18). But naturophonic intonation aims at reproduction of natural sound sources (the onomatopoeic model) that are 
external to the sound maker, whereas the anthropophonic intonation follows instinctive impulses to express a certain internal state (the infant-crying model).

Onomatopoeic reproduction can be creative - a natural prototype can be taken just as a general idea for one's explorations. Like anthropophonic intonation, it affords the dialogic arrangement. This intonation is combinatorial. One kind of naturophonic intonations can be combined with another to form a new compound, whose expression will combine the expression of both types - a clear example of this can be found in indigenous Jaw Harp traditions [531]. Although a sequence of such intonations can appear to possess pitch values and comprise a pitch contour, sound makers usually operate within the domain of timbre rather than pitch, constructing music from timbreclasses, based on the capacity of their vocal apparatus (or a musical instrument) to generate sounds similar to a selected natural prototype. Sometimes naturally-occurring human sounds (e.g., stepping) are imitated. The generated sounds are often appreciated aesthetically and can receive a crafty arrangement.

Audio-17: Aleut "The crow-song", solo imitation of a crow. http://chirb.it/3aK7Gm

Audio-18: Koryak dance-song "The ducks", female duet. http://chirb.it/Gv93de

Audio-19: Udege solo vocal imitations of birds. http://chirb.it/0x2O5a

Naturophonic intonation might be subdivided in two types: khasmatonal and ekmelic. Khasmatonal intonation is characterized by the contrast of at least two constituent timbre-classes, connected by the abrupt transition, especially if accompanied by the registral leap (Audio-19). Ekmelic intonation is characterized by the smoothened transition between two (or more) timbreclasses or pitch-levels within the same register (Audio 17). At present, there is not enough data to conclude whether these types are derivative of one another or present a parallel development.

3. Organophonic intonation adopts the sound of a specific musical instrument as a default model for melody-making, including vocal music (Audio-15). This intonation is the first to support the collective performance of music. It often distinguishes one indigenous ethnos from another by manifesting a sonic ideal of the entire music-user community. Modeling can be assigned to a percussive instrument tunable to a single pitch (e.g., tambourine for Chukchi), or to the entire instrumental family (e.g., Javan gongs, or African xylophones). A phono-instrument can be selected as an organophonic model, if it is considered of paramount importance for the community - for example, the sound of a flying arrow in Southern Siberia [138]. The latter case scenario often occurs in societies that retain animistic and totemic beliefs, where a nonmusical prototype can constitute a fetish exercising its powers through music. The very adoption of organophonic prototypes necessarily involves bonding and consolidation of a social group. Certain acoustic properties of organophonic intonations set a standard for collective musicking - very much like the concert pitch convention standardizes orchestral music of today. Organophonic standardization of a music system constitutes an advance towards abstraction of musical signs in comparison to two earlier intonation types.

Sheikin insists that through organophonic model every indigenous musical culture defines its favorite "acoustic medium" and perpetuates it in the tonal organization of some central musical genre, usually ritual (e.g., wedding, burial) and/or epic (heroic epos, tales), which becomes canonized and carefully preserved (ibid., p.38). According to Sheikin, the primary reason for the survival of timbre-oriented music in the extreme North is total absence (or scarcity) of musical instruments capable of generating multiple well-defined pitches (e.g., the Nganasans - see [122]). Once an ethnicity invents or borrows an array of pitch-oriented musical instruments, its music 
system starts shifting towards pitch-based tonal organization and collective music-making (e.g., the Chukchi - see [532]). Often a national tradition accumulates a few organophonic models throughout its history: e.g., Hellenic lyra versus Hellenistic aulos for the Ancient Greeks [474]. Timbre-oriented organophonic intonations (such as the articulation patterns of the tradition of "talking Jaw Harp" - see [28,501]) as well as the ekmelic pitch-oriented ones (e.g., bull-roarers) can coexist with naturophonic intonations, combined within the same music work (Audio-22). Emmelic pitch-oriented organophonic intonations (e.g., string and wind instruments) are usually not mixed with anthropophonic and naturophonic intonations. All organophonic intonations are easily merged with each other to form complex combinatorial and compositional structures.

Audio-20: Musicking on the Chukchi galgasinainan'an (a whistle made of a bird feather's stalk). http://chirb.it/fcr4qs

Audio-21: Musicking on the Itelmen kel-kel (a buzzer that traditionally was believed to tame an evil spirit but now is used as a children's toy). http://chirb.it/hH5h4m

Audio-22: Improvisation on khomus "The autumn sounds". Imitative and creative uses combined. Courtesy of Ivan Alexeyev. Used with his permission. http://chirb.it/6vt6f2

Here we should point out that the genesis of organophonic intonation is directly related to the issue of violence. Sheikin reports numerous accounts of the origin of ethnic organophonic models from rituals, related to killing animals either during hunting or sacrificing rites. Thus, the Mansy and Khanty clappers originally were sticks used for hunting, symbolically representing a crane; the sacred drum-log of Amur/Sakhalin ethnicities was a forest tree, hit to lurk the sacrificial bear into a trap; the Yeniseian tambourines were made from the reindeer's hide as part of the reindeer cult [138]. Even more direct are the ties between violence and such organophonic models as a flying arrow and bowed harp (ibid.). Lavergren argues that all prehistoric instruments were either closely related to hunting, looked like weapons, or constituted byproducts of hunting: e.g., whistles and reeds relate to decoys used for bird-hunting and often imitate bird-calls in indigenous music; bullroarers closely resemble bolas still used by Amazonian Indians to entrap prey's legs; horns are made of animal horns, etc. [533]. Music rituals, still performed before hunting by indigenous populations of the Amur and Baikal region, aim at securing a kill by commanding the prey through the supposed supernatural power of organophonic intonations [138]. The model of attracting the prey by means of the appropriate decoy call probably prototyped many similarities between musical and hunting instruments.

In a way, earliest organophonic intonations might be seen as a magic contract between aggression and friendship: in order to kill a prey for sustenance, a hunter must please and hopefully befriend the master-spirit that patronizes a hunted species. Noteworthy, in many modern hunting indigenous societies, rituals are called to emphasize the "non-aggressive", i.e., anger-free, orderly and harmonious character of a kill. Similar rituals exist amongst pastoral nomadic societies in regard to butchering and sacrificing domestic animals - which is paradoxically combined with attachment and care to these animals. Often, it is hard to draw a line between pastoral and hunting rituals. As Alekseyev notes: "languages of domestication" are generated by borrowing "acoustic traps and snares" (decoy calls, hunting songs, etc.) from hunters and transforming these animal-related sounds into animal-directed commands [534]. The same applies to the musical instruments used by herders to control their animals and scare off predators - here, a perfect example of organophonic wordless singing is kulning [13]. Another example is the modeling of the Croatian vocable-based singing style of tarankanje upon the piercing sound of sopile (shawm or oboe) [535]. 
4. "Metrophonic intonation" is the new term we propose to refer to the musical intonations generated by musical instruments that feature "metric" designs and to vocal imitations of such intonations. "The system of metric temperament" ("metric" - in a sense of providing a unit for measuring) was defined by Beliayev as the method of organological construction, where linear distances between the holes on woodwind instruments or frets of string instruments are placed in correspondence with the relative size of interval-classes common for that music culture [536]. Beliayev inferred this system from comparative measurements of various Eurasian musical instruments that belonged to the same organological class [537,538]. He established that the incrementality and symmetry in the design of physical dimensions of musical instruments influenced the standards of tuning and intervallic typology for many cultures. Those traditions that adopted the metric principles usually came to discover the circle of 5ths and diatony ${ }^{47}$ (as exemplified in legends of discovery the Pythagorean or Chinese lü tuning [32]). Sheikin confirms that metric standards are widespread and sometimes emerge even within music cultures that retain timbre-oriented traditions [138]. However, such standards do not always have the power to influence vocal intonation. ${ }^{48}$ Kvitka discussed the importance of Beliayev's metric theory of organo-construction for studying prehistoric music and pointed to the contribution of "optic symmetry" to the design of neolithic flutes [380]. The same principles apply to the design of Paleolithic flutes [395].

Perhaps, the earliest implementation of metric modeling in a music system was the 4-string design of a lyre, canonized by the Sanskrit hymnody of early Vedic tradition. The latter thereafter spread westward and materialized in the rhapsode tradition of the Archaic Greece, most famous in Homeric epics whose melodic pitch-formula was reconstructed by Martin West [375].

Audio-23: Demodokos' song from the Odyssey, solo accompanied by lyre (ca 8-7th centuries $\mathrm{BCE}$ ). The musical mode consists of 4 pitch-classes corresponding to 4 lyre's strings. Reconstruction by Hagel and Danek [539]. http://chirb.it/yGwq6g

Strings' length or distances between frets and holes are clearly visible to players and listeners, so that the differences in sizes or positions they see perfectly match melodic intervals they hear [39]. The rise of popularity and social prestige of tunable multi-pitch woodwinds and strings enabled the spread of sophisticated math-based tuning systems across the Middle East [474]. Especially harp/lyre helped define the tuning standards [540]. Plato left explicit explanations, why modeling intonation on string instruments is preferable over wind instruments [541]. And once the majority of musical instruments of a given culture adhere to the same metric standard, the entire music culture, including its vocal traditions, absorbs the metrophonic intonations. Mesopotamian harps and Greek lyres most likely served as pan-Eurasian metrophonic models for many musical cultures, including folk music, that looked up to their cultural achievements - from the British Isles to China (see [375]).

\footnotetext{
${ }^{47}$ According to Beliayev, in many folk music cultures, instrument-makers empirically figure out that dividing a string in half generates the octave and in $3 / 4$ - the interval of a 4 th. With these two interval-classes in place, the intervallic value of a 5th can be inferred from an octave by inverting the interval of a 4th. Once the "metric" equivalents of 5th and 4th are established, it is easy to define the value of a whole step by deducting the 4th from the 5 th. Dividing this whole step in halves gives the "metric" value for a semitone. This basic arithmetic is widely used for constructing musical instruments across the Eastern Eurasia.

48 Thus, a drumming log (a sacred instrument for a number of ethnicities of the Russian Far East) is built according to a strict ratio of size and weight, but does not prototype singing intonations. This is in contrast to Siberian indigenous flutes, constructed in observation of proportions of the distances between the holes - in this case, flutes do set the metrophonic model for singing in certain genres.
} 
The most important consequence of the adoption of a metric standard is the emergence of the institute of authored musical composition - metric standards secure accurate reproduction of the same music across different instruments and vocals. This is what separates metrophonic intonation from organophonic one. Rational incrementation in tuning of musical instruments leads to rational incrementation of interval-classes in a way that is most obvious and intuitive to every music-user.

Audio-24: Euripides - First Chorus From the Orestes "I cry, lament so much" (408 BCE) from the 200 BCE papyrus. Instrumental version. Lydian key, chromatic or enharmonic genus [542]. Reconstruction by Halaris. http://chirb.it/C1sk09

Audio-25: The same as above but vocal version. Both versions use the identical intervalclasses. Metrophonic intonation enables the choral performance - Greek tragedies were accompanied by choirs made of 7-15 performers who had to learn a new music for public performance [372]. http://chirb.it/8PBDeD

Standardization of the entire music culture leads to crystallization of a "cover" art-song, i.e., a finalized (often fixed in the musical notation, as Audio-24) and authored musical composition that can be performed by personnel in different settings.

\subsection{Stage 4: Tonality of collective music}

The uniforming power of metrophonic intonation brought to life the idea of interval-classes and the intervallic sets, materialized in the concept of a musical key. Its earliest documented implementation was in the form of the Ancient Greek theory of tonoi. However, if for modern Western musicology, key is synonymous with tonality, for Ancient Greeks tonoi differed both from musical harmonia (which was understood as a specific type of intervallic proportionality between the tones of a fundamental unit of music-making - a tetrachord) and sustēmata (melodic scales) [372]. The notion of tonoi emerged from the need to re-tune a musical instrument in order to play a certain harmonia, which created a confusion between harmonia and sustemata. In an attempt to clarify the taxonomy of tunings available to a performer, theorists introduced classification of octave species similar to our heptatonic scales, but their efforts did not have much effect on practice that was still driven primarily by distinctions between keys [543]. Octave scales did not exist outside of a key, and were not controlled for tonal gravity (Greek hestote), unlike our notion of "tonicity" [544]. Over time, key and scale each formed their own theoretic frameworks. Thus, Greeks distinguished between "modulation according to the scale", and "modulation according to the key" [474].

The notion of musical key underlies the upturn in collective music and, in a way, opposes the notion of "musical mode". In essence, key reflects the voluntary adoption of a set of rational rules, used pre-compositionally to conceive a music work, thereby enabling collective music-making according to the uniform implementation of pitch-classes and interval-classes. Most importantly, musical keys manifest the commitment of the majority of laypeople (as opposed to music theorists and teachers) to produce and consume music according to explicitly prescriptive standards. The existence of keys in a music tradition implies that music-users recognize the use of certain pitchclasses as erroneous. This is in contradistinction to multitonal modes of folk music traditions, which follow informal implicit music theory (descriptive rather than prescriptive) that knows no interval-classes and does not recognize mistakes on part of performers - whatever a performer chooses to express is "right" [545]. The musical key as though unlocks the door to involving large 
masses of people, across kins and clans, to join their efforts toward a single performance goal. This is what generates the notion of "wrongness" - pitch- and an interval-classes that do not conform to the rules of public convention become necessarily "wrong". Without this, music simply cannot be put on service for a state. The political use of music to direct proactive aggression of citizens toward some enemy relies on their familiarity with musical keys, as we shall explain below.

Musical modes usually end up subordinated to musical keys. Tuning practice encourages a single key to incorporate multiple modes in order to minimize retuning (the traces of this can be seen in Western classical music, where a minor key supports 3 modes: natural, harmonic, and melodic). The complex interplay between key and mode originated in the Ancient Greek theoretic thought [546]. The convenience of playing one pre-existing tune in one mode immediately after another tune in a different mode, without stopping and re-tuning an instrument, incentivized a performer to keep the same tuning (i.e., key) for as long as possible and make the necessary adjustments all at once during the modulation from one mode to another. This is how the idea of what we know as "chromatic alteration" was brought to life. Ancient Greeks altered their tonoi either by increasing the number of strings on harp/lyre (which was their organophonic model during the Classical period) and sparing the extra string while playing in a different mode, or, by using the fingerboard to play closed strings, as in lute. Since the number of alterations had to be minimal to make modulation as fluent as possible, performers had to carefully select a string from which to start playing in a new mode and, if necessary, to transpose a pre-existing tune to match the tuning of the strings. This practice subordinated modes to keys: modes became defined in reference to a specific key.

Keys were invented to resolve the confusion between the demands of normative tuning according to a music theory and practical tuning for the convenience of common modulations. Modal structures of two desired tunes had to fit into the set of pitch-classes, produced by normative tuning. The strategy of looking for the least number of alterations caused a performer to look for multiple fits. Manifestation of this conundrum can be found in the opposition of thetic and dynamic nomenclatures in the Ancient Greek theory, as explained in Ptolemy's "Harmonics" [546]. Thetic notation follows strings and not musical "notes" - and defines tones in their ascending order from the lowest to the highest tones of the entire ambitus available to a music-maker. Dynamic notation proceeds by the functions of tones in relation to the principal tone of the music system - the Mese (i.e., our A3). Thus, a tone other than the Mese can be qualified as the dynamic mese, if it relates to the other tones of a melody in the same way as the thetic Mese relates to its neighboring pitchclasses. In this way, pitch-classes can be identified either by "moveable" or "fixed" taxonomic names (similar to what we have today in our choice of absolute or relative solmization naming). A pitch-class moveable by position (locus) becomes renamed by function and thereby "fixed" in the modulations of the genera (e.g., making a diatonic tetrachord into chromatic).

From this, we can see that keys were invented to quantify pitch classes and infer their optimal tuning for a musical instrument and for singing (Classical Hellenic tradition relied on the monodic model of singing to one's own accompaniment on lyra). Such need is not limited to Ancient Greek and Western classical music traditions. It emerges in any octave-equivalent metrophonic musical culture that requires a performer to retune a musical instrument in order to play in a different mode [547]. Tuning always follows a rigid procedure of deriving one pitch value from another (matching the sounds of different strings). Hence, one pitch-class is always singled out, and the entire pitchset is derived from it in a chain progression of tuning. This centrality is neither about "stability" in a sense of minimal variance of frequency values, nor "stability" in a sense of "tonicity" (capacity 
to terminate a musical phrase). This is because playing a tune, tuning an instrument, and bringing music to an end are quite different procedures: the first tone of the tuning procedure can differ from the first degree of a musical mode of a tune and the last tone of a musical phrase. The first tuning tone defines not the hierarchy of tension/relaxation, but the priority in defining all intervalclasses in order to rasterize a pitch contour of a given melody.

Therefore, "keys" are not found in those indigenous folk cultures, where performers do not conceive music in terms of producing intervals of a certain value [548]. This contrast is obvious in the history of Ancient Greek music: during the Helladic period it followed the folk paradigm of rhapsode music, exemplified in Homeric epics and based on the tetratonic prosodic models (without octave equivalence), set by the tuning of lyra that accompanied rhapsodic singing [375]. However, since the Hellenic period this entire tradition was entirely ignored by all ancient writers on music, as though folk music went suddenly out of existence - superseded in importance by the rational music systems of Pythagoras and Aristoxenus. The rhapsodic tuning system was most probably deemed unworthy of attention, because it appeared irrational in comparison to mathbased Pythagorean and Aristoxenian systems. If a Helladic musician intuitively followed some uncodified oral "music theory" in reproducing a conventional melodic model (mode), a Hellenic musician had to calculate "on-the-fly" which suitable tones, modes, and tunings (key) to choose for the needed expression according to the rules of melopoeia - i.e., the "science" of emmelic composition [549]. It was the competence in such science that was respected, once the rational music systems were widely adopted.

- "Intervallic" conceptualization characterizes music cultures that commit to a single standard of quantification, which makes music-making rational and incremental. Intervallic standards are possible only at that stage of social evolution, when society develops science as a professional occupation [550]. Therefore, keys are found in those cultures, where music is professionalized, string instruments occupy the central position, and music theory becomes metrophonic, requiring formal learning [39].

Technically speaking, keys should be regarded as the prerequisite of tonality. The term "tonality" usually refers specifically to Western tonality that became theoretically formulated in the 18th century, reflecting the compositional practices of the 17th century [551]. The term "key" is often understood synonymously with tonality, vaguely distinguished by its specialization in the melodic context and reference to a specific pitch set (e.g., "C Major key" = C-D-E-F-G-A-B) versus tonality's specialization in harmony and reference to a general type of tonal organization [552]. The notion of tonality is more abstract and inclusive of all keys - somewhat like the general idea of "keyness" [551]. Yet another distinction is that tonality implies the presence of hierarchical organization, where one category of pitch-classes is subordinated to another category on a few levels, and all pitch-classes are subordinated to a single "central" tone [553]. It should be brought to attention that "key" differs from "tonality" not only because the latter acts as the container for the former, but also by their relation to gravity. Tonality necessarily requires the I degree to function as "tonic" - to be the most stable tone in a tonal system. Key is not as imperative in this respect. Organically (and historically), key relies on tuning, which can only prioritize a tone rather than turning it into a "tonic". This subtlety is not at play in many cases, but there are cultures where this distinction plays a role: e.g., the so-called "dominant keys" of Spanish flamenco [554]. Such keys can be conceptualized as part of the alternative tonality that followed its own course of development, different from Western tonality - something that can be termed "Mediterranean tonality" (see [554]). Similar cases could be made with other advanced music systems, based on 
diatonic music theory, metrophonic intonation, intervallic classes, and ethos-like semantic qualia ascribed to different keys - for example, Chinese theory of lü [555] can be considered a form of tonality.

Historically, the first society, where science was delegated to the cast of priests, in charge of supervising the use of music according to the set of codified rules, emerged in the 3rd millennium Mesopotamia and stood in clear opposition to folk music of the commoners [140]. Collective performance in Mesopotamia and Egypt included large-size ensembles and certainly differentiated between vocal melody and instrumental accompaniment, as well as between instrumental solo and tutti - as early as in the 25th century BCE, at the Mari court, Syria [556]. Ensembles of the first half of the 3rd millennium BCE included vertical harps, large lyres, small lyres, and singers. After 2,300 BCE, horizontal harps, big drums, kettle-drums, small drums, and clappers were added. The early 2nd millennium BCE saw further expansion, with addition of flutes, cymbals, and lutes, reaching sizes of up to 15-piece orchestra [557].

Collective performance on many different instruments was hardly possible without adoption of keys. The first documented evidence for standardization of the tuning procedure comes from Ancient Babylon, 15th century BCE [477]. But this procedure almost certainly was formulated much earlier, possibly implemented in the tuning of the famous Lyre of Ur, dated by the 26th century BCE [558]. The mathematical reference tablets, employed for tuning in Middle Babylon, were already known by the 26th century BCE [559]. The 28th century BCE relief depicts two lyres played together [557], which definitely required both players to share the knowledge of the same mode/key. The Middle Assyrian tablet VAT 10101 [560] contains a census of Akkadian lovesongs, classified by seven modes (tunings). There is evidence that in Babylonian temples it was customary to perform a variety of songs to accompany the liturgy, with no fewer than 6 different compositions performed by the choir and orchestra [561], which would have required at least an occasional retuning during the same performance session.

Introduction of keys enabled proliferation of orchestras, which in turn, initiated the competition between cities and states for hosting the most prestigious orchestra. Egypt competed with Mesopotamian states in the diversity and numerosity of musical instruments that were used to glorify gods and kings [562]. Early Mesopotamian cities, such as Ebla, Mari, Aleppo, and Ugarit, competed in the wealth and luxury of their music at temple and palace celebrations of military events, religious holidays, funerals, and sport festivals [563]. Possession of good musicians was regarded as a symbol of prestige, so that musicians were routinely exchanged as diplomatic gifts and taken as booty in military campaigns [564].

Not only orchestral music became the epitome of collective music performance, it, as a rule, addressed massive audiences, whether at court or temple festivities - on the scale unthinkable for earlier forms of music [565]. Thus, according to Josephus, king Solomon commissioned 40,000 chordophones (knr and nbl) for the Levites to sing their prayers [427]. Music was turning into a political tool for consolidating citizens against external enemies and illuminating the state power. The government regulated music in the 22th century BCE in Sumer, as evident from the textual descriptions of the music reform undertaken by a Sumerian king Shulgi [560]. Shulgi codified the new theory of music, standardized the technique of playing seven musical instruments, defined new religious and court genres associated with these instruments (prayers, ballads, supplications), delegated the task of disseminating this new music to scribes, and ordered his subjects to perform such music in cult-places [566]. Shulgi put in place professional music administration across his 
kingdom to handle ritual, court, military, and marriage music, keeping on payroll full-time music employees [567]. Other Mesopotamian courts followed this model [568]. From Mesopotamia, it spread to Egypt and later to Greece [569], thereafter adopted by Rome [570] and Byzantium [571573]. Mesopotamian influences reached as far as China through the Silk Road during the Roman times [574] and probably even earlier [575]. Music has become a weapon of political nationbuilding across Eurasia.

Wide adoption of standardized metrophonic modes and keys brought yet another important consequence that contributed to the rise of nationalism and proactive aggressive attitude towards the perceived threats to a nation. Each specific mode/key was believed to carry a particular ethos [Greek, 'character'] - i.e., an abstracted affective state that was supposedly projected by the sound of a specific musical mode/key on those exposed to that sound [576]. Thus, Plato in his "Republics" characterized the ethos of the most popular tonoi: virile and energetic Dorian, headstrong Phrygian, effeminate Lydian. Aristotle classified different types of ethos in respect to their affective properties in his "Politics": diastaltic ('dilated') - as heroic; systaltic ('contracting') - effeminate; and hesychastic ('sticking') - pacified. From our modern view point, Aristotle's ethos was a psychological "phenotype" - a type of human mentality developed under the influence of one's consistent consumption of a preferred type of music: e.g., Mixolydian ethos supposedly made its patrons sad and grave, Dorian - moderate and settled, and Phrygian - enthusiastic.

Half-a-millennium after Plato, views on ethos did not change much. A renowned treatise "Harmonia" by Claudius Ptolemy (fl. AD 127-145), an astronomer and mathematician from Alexandria, identified a semantic framework for each mode/key and specified the genre where that mode was most popular. Thus, stitching two Dorian tetrachords together projected courage, dignity, and sternness, so that the habitual listening to such music led to spiritual equilibrium. Joining two Phrygian tetrachords, in contrast, promoted passionate, even ecstatic states, most suitable for dithyramb. On the other hand, two Lydian tetrachords supposedly made listeners plaintive, mournful, and grieving. Mixing different tetrachords together (e.g. Hyperdorian or Aeolian modes/keys) generated states, intermediate between the ethos values of the constituent tetrachords. The changes of modes in Ancient Greek and Roman music were understood by listeners as changes of mood. Cleonides in his Eisagogé harmoniké ('Introduction into Harmony'), 2nd century AD, summarized the development of music theory of Aristoxenus, covering a span of practice of about 400 years, and defined modulation as: "modulation in melodic composition takes place whenever there is modulation in ethos from the diastaltic to the systaltic or hesychastic types" [38] - following Aristotle's guidelines.

Music was an essential part of life in Hellenic societies. It was performed on all religious and civic occasions, during banquets and friend's gatherings. Every form of Greek poetry was accompanied by music. Music was part of the curriculum in Greek public education, and it was as popular as sport. Just like Olympic games, the music-dedicated Pythian games were held consistently from the 7th century B.C. to 394 B.C., open to the general public. Everyone could compete in the art of singing and playing (including competition of choirs). A citizen of an ancient city was expected to improvise music and partake in a choir, and certainly, to appreciate the merits of music. Therefore, the ethos system was really omnipresent throughout Ancient Eucumene. In "De Instutione Musica," Boethius states that the adherents of Pythagoras used to listen to "certain melodies" before going to bed, to achieve state of relaxation, whereas reserving other "certain melodies" to invigorate themselves and "dispel the stupor and confusion of sleep" after waking [38]. To put it in perspective, we must remind that Pliny, Pausanias, and Diogenes Laertus tell us that Pythagoras' 
teaching was the most popular at his lifetime and at least 200 years after his death, and attracted more pupils than any other contemporary school of philosophy.

Although the term "ethos" is tied to the Ancient Greek civilization, its doctrine was definitely familiar to a number of preceding and extemporaneous civilizations. The roots of the Greek ethos doctrine can be traced to the Mesopotamian origin [577]. The mathematical foundation for "classic" music theories of the entire Near East and Greece seem to have originated from Babylon [578]. We should not forget that Pythagoras, recognized as the father of mathematics, music, and ethics, learned his crafts from Chaldeans (Farmer 1965). Indeed, there is a documental evidence that Babilonians recognized emotional specialization of modes/keys - thus, the qablite mode of Hurrian Hymn-6 was believed to invoke a state of joy in listeners [422].

Greek "classic" ethos theory and music theory were based on the philosophical theory of "harmonia" [576], which underlay music theories within the huge region from Greece to India [579]. The idea of ethos relied on the doctrine of global harmony (aka "music of the spheres") that was central to numerous world civilizations: the belief that celestial bodies (i.e., astronomic aspect), social fabric of states (political aspect), and human mentality (psycho-physiological aspect) were all governed by the same mathematical principles of proportionality that were expressed by the tuning system of musical modes/keys [580]. Hence, a view of music as "the sounding number", common for Antiquity and the Middle Ages [581], and the cross-cultural tradition of pairing music theory with mathematics in education and research [582] were all related to ethos. Thus, Plato's theory of mimesis postulated that musical pitch exercised psycho-emotional power over listeners in the same way as the sun or the moon, therefore engaging both music theory and math to define the "correct" order of things [583]. Along the same lines, tonal organization of music was mathematically expressed and related to social order and cosmological principles in music cultures of the Near and Far East, as well as India [584]. The dialectics of global harmony and disharmony and their connection to ethos are reflected in the music theories of raga [585], yayue [586,587], Western medieval modes [588], and maqam/dastgah [589].

In this cosmological perspective, the possibility that a musician might mistake the proper tuning threatened to upset the world's order, the affairs of a state, and the well-being of people. It was this concern that justified the institution of music administration to supervise the "correct" performance and teaching of music [568]. In the eyes of citizens of ancient civilizations, it was their mission to instill and maintain order in the regions they inhabited. Folk music cultures of neighboring nomadic people were viewed as "barbaric", compared with animal calls, and judged "wrong". Often this was taken as a justification for "correction," including the maltreatment of "barbarians." And this attitude was as strong in the Hellenic world as in remote China [590].

In essence, the institutionalization of metrophonic intonation by state authorities should be held responsible for the emergence of ethos and the ethical consequences of its administration - above all, the perceived need to guard the interests of all members of a civilized community by regulating its music usage and suppressing the intuitive unregulated, "barbaric", uses of music. It is through this dichotomy the grains of nationalism were seeded. We should point out that Plato's evaluation of modes/keys was already "nationalistic": he criticized exclusively non-Greek modes - "barbaric" Phrygian (Phrygia was located in central Anatolia) and "effeminate" Lydian (western Anatolia), while praising Greek Dorian (Doris was located in southern Thessaly - the cradle of archaic Greek tribes). The names of Greek modes/keys likely reflect the place of origin of the music from which they were theorized and hellenized or which they resembled [375]. 
The long-term practice of standardized metrophonic intonation in semantic connection to ethos is prone to put in place the "us-versus-them" mentality, potentially promoting the increase in animosity towards the neighboring states and ethnicities, especially the ones perceived as "uncivilized" or propagating a competing metrophonic model. Already in times of Plato, Spartan and Athenian music cultures supported different models of militant and ecclesiastic music, viewed critically by both sides [591]. These differences, along with the ethnic differences - Athenians descended from Ionian tribes, while Spartans from Dorian - played a role in the Peloponnesian War [592]. The use of music in such political confrontations and conflicts by no means reduced as time went on. Quite the contrary, it kept growing until in the 20th century music became a cultural weapon, deliberately employed by governments, at first in WWII and thereafter in the Cold War [593]. The belief in social powers of ethos proved to be amazingly long-living, trackable as late as in the Baroque era scholarly discourse on music [594]. The rise of nationalism in European music cultures of the 19-20th centuries, where musical traits typical for a nation could be idealized by its nationals and vilified by nationals of the hostile state [595], in essence, revived the philosophy of ethos. For this reason, we think that the entire period from Sumer to the mid-20th century can be qualified as a single evolutionary stage.

The onset of this Stage-4 most probably occurred substantially earlier than 5 kya in Mesopotamia and Anatolia. Ethnographic records, history of folklore, global data on conflict events, and largescale surveys disclose systemic links between pre-industrial societies that relied on animal herding and a culture of honor that emphasized violence, punishment, and revenge [596]. These links are traceable to prehistory and still remain vital amongst the present-day descendants of herders as well as speakers of languages associated with long-living herding traditions. The totality of available data supports the conclusion that this form of economic subsistence has generated a functional psychology that keeps playing a role in shaping conflicts across the globe (ibid.). Noteworthy, the aggressive psychological phenotype, typically associated with culture of honor, is more common amongst pastoral than horticultural societies [597].

This suggests that proactive aggression started escalating as animal-breeding was introduced, and first pastoral societies emerged. The earliest sites of animal husbandry are located in Southeastern Anatolia and Northern Iran, ca 11-10 kya, and related to proto-Indo-European populations [13] the same that were renowned for their hospitality and vendetta codes (see 6.2-6.3). Paradoxically, the tradition of pastoral music has been faceted by the extensive practice of music-like communication of humans to domestic animals, modeled after human motherese and lullabies, devoted to expressing care and instilling trust, and supported by the behavioral code of tolerance, most illustrated in the cow-worshiping cults (ibid.). Obviously, pastoral culture possessed the diametrically opposite side. A pronounced reduction in reactive aggression within a household and a clan of the pastoral society was accompanied by a noticeable increase in proactive aggression toward those outsiders who were deemed a threat of encroachment - especially, theft of domestic animals and misappropriation of grazing land.

Because herd animals constituted the easily alienable property, the mobile livestock economy became characterized by frequent clashes over herd animals. As Orton's analysis of the Late Neolithic herding practices in the Balkans demonstrates, human-animal interaction actively structured the transgenerational organization of communities, where the domestic animals engaged humans in kin-like relationships to the extent that human and animal lineages were linked together, assigned to specific households, and perpetuated over generations [598]. In this respect, herding 
societies resembled the earliest city-states by having a single authority figure and a militant personnel whose duty was to protect all human and "animal subjects" despite their differences. Moreover, animals "belonged" to humans in a way pretty similar to how slaves in ancient states belonged to their masters. The constant necessity to defend one's livestock shaped the behavioral code of pastoral societies essentially in the same way as the constant threat of assault on a slaveowing state - favoring those households that projected a more formidable impression and therefore were not attacked by the neighbors.

The tonal organization of traditional pastoral music also shows similarities with metrophonic music systems of Babylonians and Ancient Greeks. Although indigenous pastoral traditions usually do not feature musical keys, they do employ interval-classes that tend to form as a result of frequent changes in distance during the same session of musicking for the livestock - as evident from the analysis of tonal organization of Nordic kulning [13].

As a final note, we wish to refrain once again that the escalated hostilities and wars, on the one hand, and the need to exchange de-contextualized meanings and know-hows with strangers, on the other hand, forged exoteric languages. The latter should be viewed as a linguistic counterpart of metrophonic music systems. The Fig.-7 below summarizes our 4-stage model.
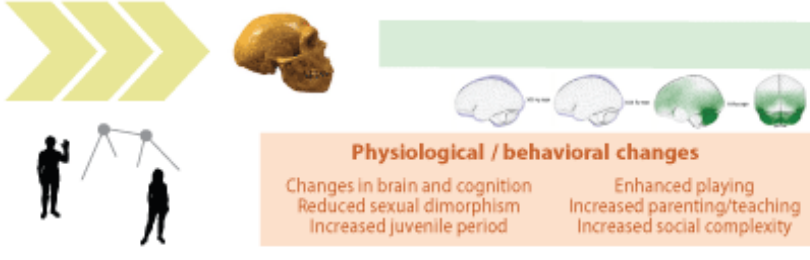

Physiological / behavioral changes Changes in brain and cognition Enhanced playing
Reduced sexual dimorphism Increased parenting/teaching Reduced sexual dimorphism Increased parenting/teaching
Increased juvenile period

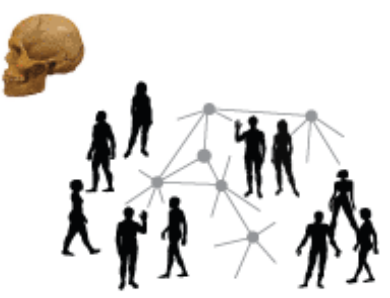

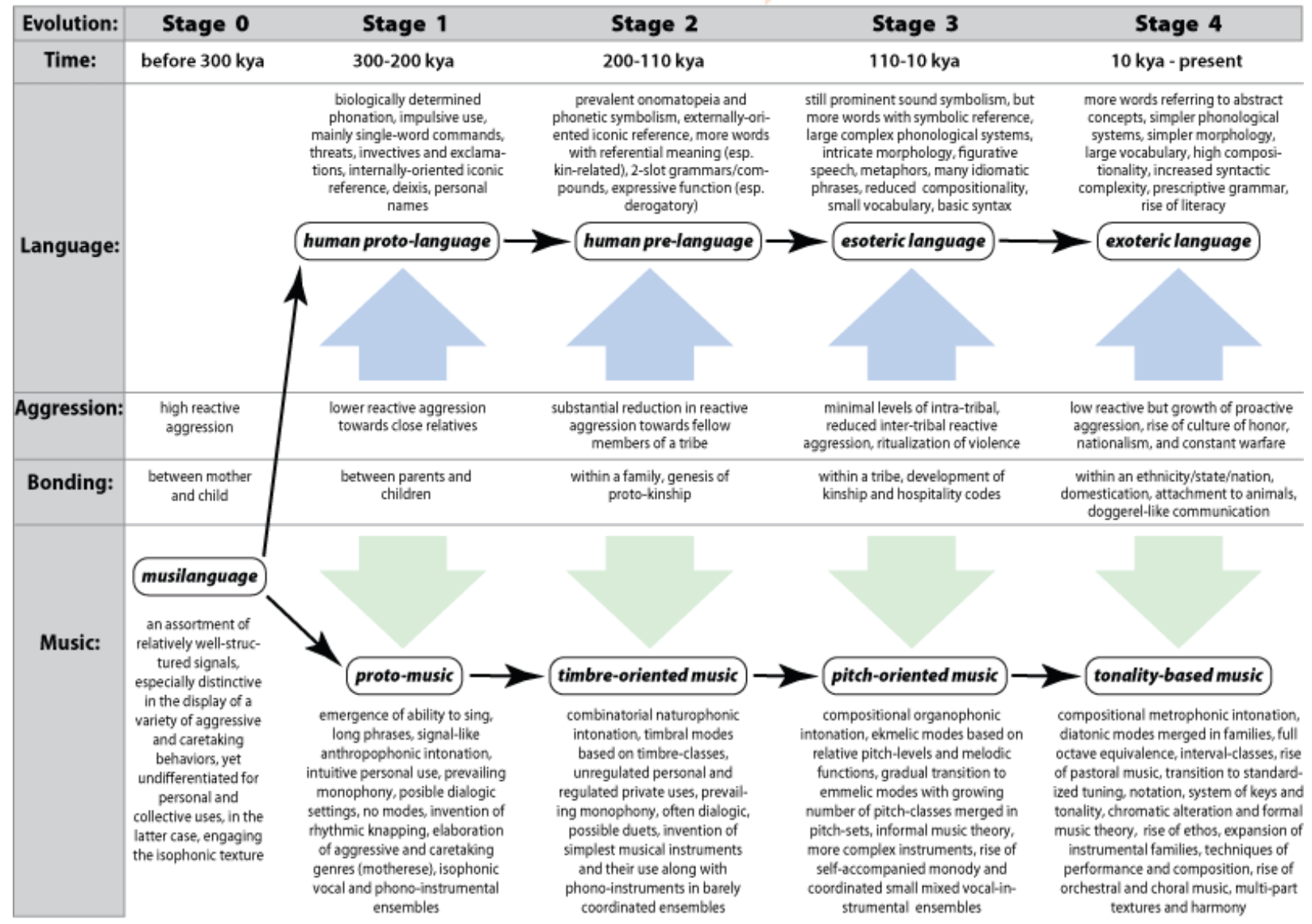


Figure-7. The timeline of the co-evolution of music and language. The figure reflects the evolution of tonal organization of music in regard to the changes in the number of music-users and their friendly/hostile dispositions towards each other. This is the original figure.

The new, 5th, stage might have been marked by the emergence of atonal music or by the recent surge in personal uses of music that followed the mass spread of digital technology (personal computers, I-pods, I-pads, and phones) and the boom of social media. Yet another indicator for the beginning of a new stage is the pronounced tendency of reduction in proactive aggression during the last few centuries [599]. However, it is too early to evaluate their contribution to tonal organization of music, management of aggression, and prosociality.

\section{General summary}

We started by defining music from the structural point of view (see 2.2), identifying its 11 aspects of expression (Fig.1), and pointing out 5 of these aspects that are indispensable for music (pitch, rhythm, meter, dynamics, and timbre). That is, the absence of structural changes in any of them indicates that the analyzed auditory material is not music, while the presence of discernable patterns in all of these 5 aspects indicates that the material under question is very likely music. Two other crucial criteria are the presence of the synchronous emotional reaction to the perceived music structures and the transposability of this reaction over the repeated use of that type of structures.

Then we listed 7 structural features of music that distinguish it from verbal linguistic communication (see 2.3). The first and the most important of them is the multi-channel nature of musical semiosis, where 9 aspects of music expression (melody, harmony, rhythm, meter, articulation, dynamics, texture, and music form) simultaneously employ idiomatic patterns to express affective states, attitudes, characters, and actions. Multiple channels enable music to convey contradictory, vague, and unstable ideas that are hard to verbalize. The second important difference stems from the first: by default, music integrates multiple elements into a single percept, both simultaneously and successively. This is manifested in music's compositeness (number of items simultaneously combined), voluminousness (number of performers engaged in production of the same item), and lengthiness (number of items grouped in succession).

The third marker of music is quite special - a unique combination of high contemplativeness and redundancy (relatively low sequential informational load) with multifunctionality (capacity to divide information in multiple channels and deliver it in parallel streams). This paradoxical design allows music to prolong the exposure of a complex affective state or the process of its metamorphosis, giving enough time to listeners to experience that state.

Thematicity constitutes the fourth uniquely musical trait: people recognize a music-work by its musical theme that conveys an image, character, or musical emotion, central to that work. The other phenomena peculiar to music are absolute pitch (both concert pitch reference and qualia of specific keys), intervallic typology (the structural uniformity within a chosen manner of distinction between different pitch levels), and general orientation towards euphony, manifested in the universality of musical modes - i.e., the cross-cultural practice of matching musical sounds in a 
concordant way to support a desired expression (even when the expression is discordant - e.g., anger or fear). Still, it should be noted that in many cases these distinctions from oral language might be not clear-cut and exist within a continuum.

Structural features specific to music determine the ways it can be used actively (performance) and passively (listening). We reviewed the most common uses of music and broke them into 14 operational types (see 2.4) that represent how humans interact with certain types of music structures across different cultures (Fig.2). In the regressing order of commonality in present-day cultural life, these types are as follows: hedonistic stimulation, emotional communication, emotional regulation, interpersonal bonding, coalition status display, compliance to norms, recreation, self-promotion, physical aid, learning aid, contemplating an event, calling to action, conservation of memories, and personal profiting. We noticed that many of these functions form pairs of dependencies, where one function cannot operate without another function already being operational. We then rearranged the order of functions to place each determined function after the determinant one. Some functions had double and even triple dependencies - which was more pronounced in the beginning of the entire dependency chain. To establish the ultimate order for such intricate connections (see 2.5), we followed the Vygotskian approach to sociogenesis [3,4]: establishing which operational function builds the foundation for the next (higher order) function that is called to support a new mode of interaction with the cultural environment. At the beginning of this progression, we placed the earliest ontogenetic functions (also common for vocal communication in the animal kingdom). Our rationale was that the ontogenetic line of development here would reflect the evolution of music from proto-music and musilanguage (since cultural phenomena can exist in no other way but via the transmission from one generation to another, on the individual basis).

We adopted hedonistic stimulation as a primordial function, because of its fundamental role for all uses of music and presence even amongst numerous animals (2.5). The other functions, ordered according to their dependencies and ontogenetic succession, formed developmental phases, where some functions became coupled within the same phase. Thus, phase-II consisted of two parallel functions: "learning aid" and "interpersonal bonding," both undifferentiated from language, and therefore likely to characterize musilanguage. Phase-III, "recreation," represented the ontogenetic stage of "musical babbling" - the earliest form of differentiation of music and language (opposed to "verbal babbling") and representative of the proto-music in the evolution of music. Phase-IV again consisted of two parallel functions: "physical aid" and "emotional communication." Both of them, as well as their parental function of recreation, might potentially be carried by speech, although not as effectively as music, which putatively joined them into proto-music. However, the next, 5th phase, "emotional regulation," was more of a specialty of music rather than speech intuitively used by children to adjust their emotional state and the state of imaginary protagonists of their pretend-games according to the demands of a social situation. This prompted us to regard this phase as transitional from proto-music to music. Phase-VI, "compliance to norms," closed our model of the early childhood period, characterized by the greatest uniformity of features across different populations (due to its reliance on the biologically ingrained mechanisms of processing music).

Phase-VII, "coalition status display," ontogenetically corresponded to the common adolescent trend of seeking membership in a peer-related social group outside of a family circle, while culturally corresponding to the tradition of "personal song" (see 5). This let us qualify phase-VII as intermediary between the genetically-controlled and culture-controlled functions. From there 
on, phases became decidedly culture-specific. Phase-VIII again consisted of two parallel functions, "conservation of memories" and "self-promotion," inherently connected to both sides of the "coalition status display": indigenous personal song (e.g., singing the personal songs of one's parents or the so-called "boasting" personal songs) as well as modern peer culture. Both functions appeared no longer cross-cultural and involved abstraction: "conservation of memories" retrieved memories of something currently absent, whereas "self-promotion" aimed at showing off one's musical skills to win public prestige, which demanded abstraction of musical techniques. PhaseIX also implemented two functions: "contemplating an event" and "personal profiting." Contemplation required further advance in abstraction: music idioms had to refer to objects, properties, and events that the listener had never actually experienced. "Personal profiting" had to do with the higher order abstraction of expressive means (profiting from music is related to professionalization of music-making, competition, and originality, focusing on means of expression per se). Phase-X, "calling to action," presented the highest order of abstraction for music, when a specific music idiom was utilized in a manner similar to words in spoken languages - as a command for a specific action (e.g., military calls) that completely relied on the knowledge of conventions unique to a given culture.

Once we formulated the succession of operational functions, it became clear that most disagreements between scholars of the evolution of music had been caused by focusing on the functions grouped at the beginning, middle, or end of the entire succession chain, while ignoring the other groups. This, combined with the lack of clear structural definition of music, had made scholars often talk past each other. Using a few examples, we demonstrated the absolute necessity to consider specific structural attributes of music in the scope of its entire evolutionary development for any discussions related to musical behaviors, skills, musicality, etc..

The next problem that we approached was the issue of structural stability of music idioms at different developmental phases in the evolution of music (see 3.1). We identified 10 phases. They all were characterized by the gradual expansion of the sphere of music usage from private ("duetic" and "solitary" of phases I-III) to collective, at first family-oriented (IV-VI), then family-andfriends (VII-VIII), and eventually an entire community (IX-X). The clear trend of increasing numerosity posed the question of its contribution to the evolution of music. In quest for an answer, we looked into the specifics of transmission of music.

Experimental linguistic studies of multi-generational learning and communication have established that human communication is distinguished from animal communication by the presence of combinatoriality and compositionality. This has been corroborated by experiments involving the transmission of artificial languages via iterated learning, as well as by the analysis of the emergence of new languages (e.g., village sign languages). This tendency equally applies to music systems. The experimental demonstration of how continuous sliding sounds become broken into discrete recombinable elements at the end of a transmission chain comes remarkably close to the transformation of ekmelic (i.e., based on indefinite pitch) music systems into emmelic (definite pitch), observed by ethnomusicologists in numerous indigenous music cultures of Siberia and the Russian Far East. The process of "natural selection" for clear-cut elements is fueled by the dialectics of the diametrically opposite goals of reception and transmittance of sounds. The receiver tends to simplify a perceived pattern in sake of easier learning, while the transmitter - to complicate it to increase its expressivity. The outcome of this repetitive interaction in every link of a transmission chain is the increased compressibility of the encoding and the regularization of the variables. Consequently, new structures are synthesized by the end of a transmission chain. 
However, the voluminosity of music makes its compressibility and regularization more complex compared to language. The greater the number of participants in a music act, the greater advantage a transmitter obtains over the receivers, which prioritizes expressivity over learnability. The manifestation of this tendency is most obvious in patterns of music consumption within professionalized music cultures: large audiences often appreciate those music works that well exceed the capacity of an individual listener to fully comprehend them. The reverse holds true as well: in the smallest settings of 1-2 participants, expressivity is sacrificed to learnability, since there is no pressure to impress the audience or convince anyone of the actuality of music emotions. The systematic increase in the number of receivers usually leads to the emergence of "music rhetoric" - i.e., the art of emotional persuasion of listeners by musical means, which necessarily focuses on the expressivity of music at the expense of its learnability. As a result, music transmission in cultures with predominant personal and private musicking tends to feature high compressibility and regularization that effectively conserve music structures, keeping them simple while severely limiting (or even preventing) their diachronic transformation. This makes music communication egalitarian: all members of such communities, disregarding their differences in musical skills, actively engage in production of music. Music transmission in cultures, characterized by mass consumption of music, in contrast, promotes high rates of innovation and complexity, which leads to segregation of the community into those who are musically capable to follow new complexities and those incapable to do so. Subsequently, music communication becomes elitarian: the active production of music is reserved for the musical elite, while the rest of the society become passive consumers.

Yet another important formative feature of music transmission is that it, as a rule, introduces and amplifies cultural biases (see 3.2). Members of music communities tend to favor one structural feature over another due to the combination of factors, such as the ease of mental processing, the popularity of a feature, and social prestige associated with it. Once again, the extent of the formative power of cultural bias depends on the numerosity of participants in a music act. Personal and private forms of musicking are characterized by stronger biases, most evident in cases of appropriation of a musical model from a foreign culture. It is not uncommon to observe a radical distortion of an intervallic typology of an original tune, once it was adopted and passed through the transmission chain in a musical tradition that cultivates a different music system (e.g., a popular heptatonic song can become pentatonic). More populous music communities tend to be less "corrective" in regularizing foreign models according to the native standards, perhaps, due to greater variance in musical preferences between numerous members, which transpires in more pluralistic attitudes and greater attention to idiosyncratic features of the original.

Our overview of the recent experimental studies of the effect of multi-generational transmission on structural features of music confirms the presence of bias-based cultural distortions introduced into completely stochastic models (3.1-3.2). The pronounced discretization of elements and their grouping into culturally familiar patterns has been found in the domains of pitch and rhythm. Moreover, the experimental format of self-transmission (equivalent to the settings of solitary musicking-for-oneself in indigenous cultures) was found to deliver more idiosyncratic rhythmometric organization than in person-to-person chains, probably due to the absence of the "averaging effect." This suggests that metric regularity (comparable to prosodic feet in poetry) that is common for most forms of music of today is, at least to some extent, a product of strong demographic expansion. 
To address the limitations of experimental settings adopted by researchers on transmission of music (they tested only transmission by oneself and person-to-person), which oversimplify the real-life cultivation of music, we formulated and characterized 5 different formats of transmission (see 3.3), known to ethnomusicologists: "circular" (self-communication), "linear" (person-toperson), "tree" (person-to-group), "inverted tree" (group-to-person), and "hybrid" (group-togroup). Then we modeled the complexity of the interplay between these formats and the operational functions of music, defined earlier, using the example of the melodic intonation of a descending 3rd, characteristic for lullabies. We tracked its transformation into the so-called "universal chant" of children, cross-culturally employed in playing and teasing, and its artistic arrangement in the 1938 jazz hit-song "A-Tisket-A-Tasket" that portrayed a cute little girl.

The entire transformation involved the increasing number of participants via 19 transmission links that engaged 11 operational functions, typical for folk traditions within present-day Western societies (Fig.3). Some of these functions were used repeatedly, with variation in use. Some functions changed their transmission format. This was accompanied by the gradual reduction of active uses (production) and the increase of passive uses (consumption) as the number of participants grew. In parallel, utilitarian functions (emotional communication and regulation) gave way to aesthetic (appreciation of music) and social functions (prestige). The interplay between the functional and quantitative changes transpired in the progressive expansion of the initial "microculture" of a dyadic relation between mother and child until its transformation into a "macroculture" of thousands of people within a complex modern society. The emergence of the musical idiom, embodied in the theme of the jazz composition "A-Tisket-A-Tasket," came as a sort of "natural selection" of a series of successful "adaptations" of the initial lulling musical intonation to new uses with new participants, accompanied by "extinctions" of those variants that failed to do so. Our model of the genesis of a musical idiom illustrates the formative power of transmission formats, numerosity of participants, and functional typology in shaping music structures and their conventional meanings.

The complexity of music transmission in real-life conditions is responsible for the difficulty of identifying typologies of music structures (music lexicons) and rules of their combinations (music grammars). Unlike languages, music follows marginally fewer rules that are generally not strict. The reason for this is that music specializes in prolonged exposure of a musical emotion - in contrast to languages optimized for quick delivery of specific referential information. Optimization for prolonged exposure lowers the demand for accurate and timely encoding/decoding. Musical semiosis focuses not on the micro-level of musical elements but on the macro-level of sections of music form: combinatorial rotations of intonations and motifs do not really change the meaning of a musical phrase, whereas compositional rotations of sections in a music form drastically alter the expression of a music work (e.g., from happy to sad). ${ }^{49}$ Syntactic elements of music just "color" the musical theme with hues and tints, rhetorically elaborating and emphasizing a character of music to make it more convincing for the listeners - rather than adding up radically new pieces of information. That is why only those structural elements of music that are common for themes possess well-defined syntactic and semantic features (often comparable to words of language e.g., the melodic idiom of "fanfare"). The supportive musical elements are much vaguer. Long

\footnotetext{
${ }^{49}$ For example, Grieg's piano miniature "Homeward" op.62 No.6 is composed in the ABA form, where "A" is happy and " $\mathrm{B}$ " is sad. The entire piece sounds positive, expressing how a protagonist misses home while conducting a happy life. Replacing the sections would invert this expression and project suffering, only temporarily relieved by a hope for something better - as in another piano miniature by Grieg - "Homesickness" op.57 No.6. Note the difference in titles assigned by the composer.
} 
transmission chains increase the discrepancy between thematic and non-thematic elements, since participants with less developed musical skills tend to focus only on the theme, disregarding all supportive structures. The more of such participants are engaged in a chain, the greater the difference between the syntactic elements, specializing in themes, and the rest of the elements.

Yet another peculiarity of music syntax is its overall propensity to integrate elements consecutively - forming phrases much longer than verbal phrases - and concurrently, across different aspects of expression and multiple parts (which finds no analogs in languages). This aspect of musical combinatoriality seems to originate from the inherent cross-modal (synesthetic) connections of music: its melodic, rhythmo-metric, textural, articulatory, and tempo features are readily perceived in terms of specific patterns of physical motion (running, walking, leaping) and visible (big, small, far, close) or tactile properties (firm, soft, sharp, dull) of common environmental objects. Many musical elements have clear onomatopoeic nature and require little or no knowledge of cultural conventions for their encoding/decoding. Most music idioms are iconic and contain at least one structural feature that is shared with their typical semantic value (e.g., ascending intonation increase in energy), which greatly simplifies implicit learning of music syntax. Therefore, in contrast to language, learnability tends to be overall less important for music transmission compared to expressivity (for private as well as collective forms of music).

However, transmissions of music with fewer participants seem to result in the less transparent signal-mappings, promoting greater iconicity and smaller referentiality. Perhaps, for this reason, music cultures that are predominantly personal and private (family-oriented) manifest the "deictive" traits - in order to learn their music grammar, an outsider must be physically present in a music community to observe all objects and events that can supply the cross-modal features of music structures. This is in contrast to music cultures, characterized by great numerosity of participants in music acts, professionalized music, and codified music theory. Music elements in such music systems can be learned by outsiders distantly and formally.

General iconicity of music is yet another reason for syntactic and semantic vagueness of music elements: music-users routinely figure out semantic values of unfamiliar, forgotten, or unusually combined ("metaphorical") music elements with the help of the "textual" context, prompted by the familiar thematic idioms and music genres. The latter are especially important for "ritualizing" specific uses of music that specialize in certain semantic content. Thus, regular repetitions of descending minor 3rds with the accent on the higher tone in the binary meter produce the lulling impression, whether this pattern (musical idiom) is used in a lullaby, in a symphony, or in a pop song. This is a result of long-term habituation to the sound of this pattern in the context of a "music act" of a mother lulling a child. Once musical idioms are forged through conventionalization of a music genre, they remain quite "literal" (e.g., if a comedian uses the lulling idiom sarcastically, there is no way to figure this out by listening to the sound recording of his singing alone). Perhaps, this higher idiomaticity of music (in comparison to language) is called to increase music's intelligibility by compensating for music's propensity to simultaneously merge structures of different aspects of expression, which inevitably makes the meaning of a music work rather vague, hardly definable in words. As a result, even well-defined music idioms (e.g., fanfare) are more homonymic than words of language. Here we have to underline that inherent semantic and structural vagueness of music elements does not make music fundamentally meaningless and indiscrete. Native music-users as a rule have no problem intuitively learning the syntax and semantics of their traditional music. 
Armed with the structural definition of music, its functions, and transmission formats, we critically examined the current putative views on the origins and evolution of music (see 4). We identified two opposing types of theories: exaptationist accounts that held music for a non-functional byproduct of some pre-evolved ability - versus adaptationist accounts that viewed music as a social tool of enhancing human evolutionary fitness by establishing bonds and consolidating social groups. The adaptationist theories, in turn, featured two approaches: more prevalent "external," which emphasized the power of music to promote group coherence by attuning the affective states and behavioral goals of individual members, and less common "internal" approach that focused on the power of music to support individual management of emotions and personal well-being. Some theories conflated both approaches. We sided with the adaptationist critique of the exaptationist theories and with the combined external/internal adaptationist approach to music. However, we lined up the evidence against the conviction shared by most adaptationist, as well as exaptationist, theories - namely, that music could be defined as a collectivist tool designed to instill interpersonal bonding. Such a definition would fall short of explaining a long history of battlefield music designed to scare the enemy, the wide global geographic distribution of monophonic music (greatly exceeding that of the multi-part music - see Fig.4), and the solitary format of the earliest ontogenetic form of active musicking (music babbling during the 2 nd year of life - a universal developmental phenomenon).

Based on the available ethnomusicological knowledge of traditions of personal music, the ontogeny of the acquisition of singing abilities in early infancy, and the research on transmission of music, we concluded that the prosocial power of music was not its fundamental property but just one of the options in the array of its operational functions - a capacity that emerged at a later point of evolutionary development of music. It was not music that made humans social. Exactly in reverse, it was prosociality that enabled humans to make music. The gist of this can be observed in the fact that human newborns first establish the bond with their mothers, then learn to communicate by glances, mimics, gestures, touch, and sound - and only thereafter start musicking. The duetic vocalizations between an infant and a mother lay down the model for all subsequent phases of child's musical development, musical operational functions, and transmission formats. This way, the entire realm of evolution of music is built upon the initial pre-musical rapport, trust, and care, without which neither music nor language are possible. In order for two prehistoric hunters to attempt to speak or make music together, they first and foremost had to already have a friendly disposition towards one another - and this disposition had to be passed on to their posterity over a geographic area large enough for the new method of communication to have chances of surviving over multiple demographic bottlenecks. The emergence of conspecific sympathy must have preceded the emergence of music and language.

The cross-cultural tradition of personal song that survives amongst indigenous populations of northeast Eurasia, North America, and Greenland throws light on what the intermediate stage between animal communication and human music might have been like (see 5). Akin to "territorial" and "species-specific" songs of many animals, personal song marks the membership in an ethnic community and a place of origin for an individual member. The latter is especially informative for marriages, since in most indigenous societies of this wide region there is a custom of taking a wife from a neighboring ethnic community, which probably reflects the empirical realization of the negative consequences of incest.

Personal song also reveals its owner's kin, parentage, age, and serves as a personal identifier, similar to a passport. The set of taboos thoroughly guards against the "illegitimate" use of someone 
else's personal song. The victim of the abuse (or his close relatives) is entitled to punish the offender, who is likely to be ostracized by the entire community (which might bring dire consequences for those living in harsh Nordic conditions). Even more fearful for the indigenous populations appears the prospect of being punished by the supernatural forces believed to patronize a kin of the song-owner. Collective enforcement, retained until the middle of the 20th century, makes personal song the "credible signal" par excellence, exceeding the extent of "honesty" in animal calls. The latter are commonly abused and exploited by other animals, generating the "arm race" between the honest and dishonest signaling. Honest signalers have to keep renewing their signals to curb dishonest uses. In contrast, the tradition of human personal song is renowned for remarkable conservation and longevity. Many personal songs become perpetuated through heroic epos or via attribution to deities or important spirits. Indigenous users remember the accidentally heard personal songs for decades and know personal songs of their relatives and ancestors up to few generations.

Timbre-oriented personal song is a very likely candidate for how the proto-music might have sounded, explaining how the latter linked musilanguage with music. On one hand, personal song shares with animal communication the functionality of identifying species/ethnos, regional subspecies/kins, and behavioral type/temperament, useful for marking territory and for mating. Like calls of some birds and primates, personal song utilizes combinatorial morphology: novel songs can be generated by recombining elements of pre-existing songs (ancestral or territorial). On the other hand, although many ethnicities do not consider "personal song" music per se, their personal songs are often acoustically indistinguishable from their proprietary musical genres (e.g., Samoyedic boasting drinking songs or autobiographical songs). Many personal songs are actually "composed" by parents after days of deliberation and weeks of observing the behavior of their newborn to satisfy the goal of capturing the child's character in music.

Long history of enforcing the "honest" use of personal song might explain the inherent honesty of musical communication in indigenous traditions, where a person is believed to speak for himself, whether producing an original piece of music or reproducing a pre-existing song (e.g., impersonating an epic protagonist in a musical tale). In the latter case, listeners believe that the singer temporarily becomes possessed by the impersonated character. Such a belief system might have provided a solid foundation for the default "honesty" in later evolutionary development of collective forms of folk singing. The collective format per se precludes deliberate "lying" through music, since the latter expresses emotions, attitudes, and ideas, shared by all participants. Using music to make the audience experience an emotional state that the performer does not actually experience is a relatively novel historic development, peculiar to the Western classical music and closely related to the emergence of commercial forms of popular music.

Increased prosocial behavior distinguishes humans as a biological species (see 6.1). Neurological mechanisms regulating aggression make humans less emotionally reactive and more tolerant of strangers in comparison to extant primates and, presumably, extinct hominins. Human prosocial traits resemble the suite of features associated with domestication. Animals, domesticated by humans via selection for tameness, differ from their wild cousins. The "domestication syndrome" seems to be rooted in the endocrine and neurophysiological differences from wild animals. What distinguishes human domestication-like traits is that they must have emerged naturally, without the agency of another species (unlike the animal domestication by humans). For this reason, we prefer to replace the term "self-domestication" with conspecific sympathy: i.e., the default tendency to "like" other representatives of the same species upon the first encounter. This tendency 
seems to have been absent in the earliest hominins, emerging possibly in the Neanderthals. And it is still in the process of evolutionary development, determined by the phenotypes of the aggression management. Two pronounced evolutionary patterns are the reduction in reactive aggression, followed by the increase in proactive aggression (which seems to be on decline during the last few centuries).

The origins of conspecific sympathy remain a puzzle (see 6.2). Numerous possible causes have been named: the rise of community living, co-parenting, and collective foraging. To them, we would like to add the climate deterioration: vulnerability to climate change is one of the causes of hominid extinctions, and harsher environments are known to promote intergroup tolerance. Ethnographic accounts link life in harsh ecosystems with the presence of hospitality codes that oblige settled inhabitants to provide shelter, food, and entertainment to strangers, no matter how hard this might be. Such hospitality codes, usually supported by religious beliefs in a reward for hosting and a punishment for violation of hospitality, have been reported in a vast area of the Northern hemisphere, suggesting their environmental origin rather than cultural heritage.

Moreover, historic documents describe very similar codes, supported by similar religious beliefs, across Italy, Greece, Asia Minor, Palestine, Egypt, and India, tracing it to the Indo-European sources, reflected in sacred Vedic texts. Interestingly, the latter contain references to the ritual of wife-lending by hosts to their honorable guests. Similar practice is known amongst the Polar Girdle peoples (Eskimos, Aleuts, Chukchi, Yukaghirs, Orochi, and Maritime Koryaks) as "hospitality prostitution." The indigenous informants usually explain this and another closely related custom of "wife-trading" by the need to protect oneself from violence: the surest way to avoid aggression is to become related to a potential aggressor, which is the easiest to achieve by marriage - hence the tradition of offering one's wife to the most dangerous unrelated men. The strategy of protecting oneself by building a network of relationships, thereby reducing the number of "strangers" capable of assault, finds remarkable similarities in ancient Greco-Roman and Judaic literature. The paradoxical lumping of hospitality and hostility together is reflected in the etymology of many Indo-European words and in the hospitality law of philoxenos ('xenia'), aimed to protect one from the likelihood of stranger's assault by the reciprocity of demonstrating the host's will to protect the stranger. The dichotomy between the simultaneously present laws of hospitality and vendetta is observed in cultures from the Mediterranean and the Caucasus to Kamchatka and can be traced to Antiquity, possibly going back into prehistory.

The notion of hospitality relies on the distinction between "related" and "non-related" conspecifics, defined by kinship. This distinction is closely associated with the aggression inhibition towards the closest relatives. The lack of archeological evidence makes it hard to date the emergence of kinship (see 6.3). Many non-human primates recognize parent-child relations in their mating and somehow distinguish between "us" and "them." What separates them from human relationships is a small contribution of parental ties to daily life and social affiliations. The deciding factor seems to be a demographic influence of the environment: non-human primates live in large groups in forests, whereas early humans lived in smaller groups in open-space environments that required long-distance ranging - like the indigenous populations of the Polar Girdle. Even if such communities grow in size, their members naturally break in smaller packs to hunt and gather, since food supply rarely permits the engagement of the entire community. Membership in the ranging groups promotes kin-like affiliations. Harsh climate further pressurizes rangers to frequently form small-group alliances according to skills, experience, and specialization, and rotate in their membership. Rotation requires affability towards newcomers. This could be the mechanism for 
the genesis of conspecific sympathy and prosociality. In hard-to-survive conditions, facing the threat of extinction, land-holding and fighting for limited resources are ineffective long-term strategies, prone to further reduce population, cripple effective hunters, and create vendetta. The adoption of the "tit-for-tat" strategy and the default aiding attitude would make a more viable strategy of survival here.

The institute of personal song presents an ideal tool for transforming the rudimentary kinship into the fully-fledged family with ancestral lineage (see 6.4). The capacity of a personal song to mark a specific individual in the community is invaluable for the formation of strict codes of hospitality. The latter is modeled upon the projection of blood relation onto outsiders. Accordingly, personal song is instrumental in widening the circle of relatives, while distinguishing between close and distant relationships. This is the key feature that sets human understanding of incest apart from that of non-human primates who recognize only a mother/child relationship.

Siberian personal song, especially its implementation in the hard-to-reach Taimyr (populated with Nganasan, Nenets, Enets, and Selkup ethnicities), might constitute a part of the uninterrupted cultural tradition from at least the Neolithic times, as the archeological, paleobotanic, and paleolingusitic data suggests. There is evidence of human presence in the Ob' River area and the Yenisei Bay ca 47 kya. The Ust'-Ishim man is the oldest Homo sapiens that retained the complete genome, the analysis of which allowed the geneticists to place him in the first wave of humans that migrated out of Africa eastward. Modern indigenous Siberians share 38\% of their ancestry with the Ust'-Ishim man.

We can track personal song back to ca $20 \mathrm{kya}$, using the geomusicological and paleoclimatic data (Fig.5). North American and northeast Eurasian indigenous populations share remarkable similarities in the usage of personal song, combined with the solid evidence of gene transfer from Siberia to Alaska. The comparative genetic analysis of the ancient DNA reveals that the Yana RHS inhabitants (31 kya, near the coast of Laptev Sea, in Yakutia), diverged from the Western Eurasians about 38 kya and likely migrated towards North America along the coastline. The alternative route for the West-to-East migration was via the tundra corridor that connected Western Europe to Beringia and provided a route for the animals to escape from the harsher Western cooling to the milder climates of Southern Siberia and the Amur/Aldan region. Human hunters followed their prey. After the Ancient North Eurasian population (e.g., Ust'-Ishim man) split into Western (e.g., Sunghir man) and Eastern Eurasians (e.g. Tianyuan man), ca 40 kya, the Ancient East Asians (e.g., Devil's Gate Cave inhabitants) repopulated the northeast between 20-11 kya, forming the new admixed population of the Ancient North Siberians. Their descendants, Ancient Paleosiberians (ancestral to modern Koryaks and Itelmen) are represented by the Duvanny Yar individual, near the Kolyma river (ca 10 kya) - the closest ancient relative to the ancestral Native Americans. Ancient Paleosiberians migrated towards Beringia, crossing it most likely between 23-19 kya, when the water level was at its lowest, and definitely before $12 \mathrm{kya}$, when Beringia became submerged. The presence of human traces in the pre-Clovis sites across North and South America, the oldest of which are dated $15.5 \mathrm{kya}$, as far as in Chile, suggests that the crossing of Beringia occurred at least a few millennia earlier.

The Altai-Sayan mountain system lies at the crossroads of a number of the evolutionary important distributions: it marks the southern border of populations that have used personal song (the Kamasins and Kotts), it is the probable place of origin of the Turkic vowel harmony [600] that resembles timbral modes of the Altaic indigenous music, the motherland of the ancestral 
population that started migrating to the Northeast ( $25 \mathrm{kya}$ ), the stronghold of the peculiar clan system of seok, and the place where Neanderthals, Denisovans, and early humans met and interbred, with the gene flow going in all directions (Fig.5). We argue that the genesis of kinship, personal song, and cross-cultural hospitality codes accompanied the formation of different structured populations at the Altai-Sayan region.

Once the social infrastructures and the interrelations between the structured populations across Eastern Asia and North America were settled, the development of local musical and linguistic forms of communication (specifically, esoteric languages) became possible (see 6.5). The interpersonal uses of music probably instilled friendly personal relations, stimulating the development of languages, making them at last autonomous from music. However, languages might have retained connection with music through their vowel systems. For music, the evolution went towards conversion of timbre orientation into pitch orientation, followed by gradual increase in the number of pitch-classes in musical modes. The earliest pitch-oriented modes probably contained 2-3 pitch classes, which corresponded to linguistic vocal systems that contained 2-3 vowels. Examples of such correspondence can be found in the tritonic Andean traditions of the northern Argentinian religious trumpet music and bagualas songs, Atacama songs of northern Chile, and the Q'eros music that is believed to be a remnant of the Inca civilization - corresponding to 3-vowel systems of Quechua and Kunza. Another example is the correspondence between the Inuit 3-vowel languages and their tritonic music tradition. Notably, both examples belong to the ethnicities of the Paleo-Siberian descent (related to personal song).

In Soviet/Russian ethnomusicology, the following progression of schemes of tonal organization has been established by etic as well as emic comparative analysis of music works (Fig.6):

1. Timbre-oriented modes - a multitude of timbre-classes (akin to linguistic phonemes), interconnected according to some mutual feature(s) within the entire vocal range of a voice;

2. Khasmatonal modes - indefinite pitch contours in 2-3 contrasting registers within the entire vocal range of a voice, connected by abrupt leaps;

3. Ekmelic modes - 1-3(4) pitch levels of variable stability in frequency values, enclosed within 1 register, < octave, smoothly connected by steps of indefinite pitch, of varying size;

4. Oligotonal modes - timbrally marked definite pitch-classes within 1 register $\leq 1 / 2$ octave, separated by discrete steps: monotonic - 1 definite pitch-class, ditonic - 2 pitch-classes (1 definite, 1 variable), tritonic - 3 pitch-classes ( 2 definite, 1 variable, or vice a versa);

5. Mesotonal modes - definite pitch-classes, abstracted from timbre, enclosed within 1 register $\leq$ octave, separated by discrete steps and small gaps, melodies can contain leaps: tetratonic - 4 pitch-classes, pentatonic - 5 pitch-classes, hexatonic - 6 pitch-classes;

6. Multitonal modes - well-defined pitch-classes, enclosed within the ambitus of 1-11/2 octave, separated by steps (gapless), melodies with steps and leaps: non-diatonic heptatonic - 7 pitchclasses, some octave-equivalent; octatonic - 8 pitch-classes, non-octave equivalent;

7. Diatonic modes - fully octave-equivalent pitch classes, defined through the circle of 5ths and interval-classes, enclosed within the ambitus of about 2 octaves, using a single tonic: heptatonic - 7 pitch-classes with chroma, pentatonic - 5 pitch-classes with chroma;

8. Chromatic modes - diatonic pitch-classes can be altered according to specific rules, alterations are octave equivalent, expansion of the ambitus $>2$ octaves.

The entire line of development is characterized by the increase in complexity due to the discretization of the growing number of building elements. This is accompanied by the expansion 
of the overall frequency range available for music-making, by gradual transition from heterarchical to hierarchical organization with the increasing number of levels, by the genesis of interval-classes, and by the growing complexity of textures: monophony -> monody -> heterophony -> polyphony (for European chromatic music of the Middle Ages). From the semiotic perspective, the progression of all 8 types of modes corresponds to greater and greater levels of abstraction that require knowledge of cultural conventions. This is in contrast to the prevalence of onomatopoeia in timbre-oriented modes, which supports cross-cultural communication. The landmarks in the evolution of musical modes are the following:

- The genesis of pitch orientation occurs in ekmelic modes.

- Pitch-classes emerge in ditonic emmelic modes.

- The transition from indefinite to definite intervallic typology occurs in tritonic modes.

- Syntactic organization is formed in tetratonic modes.

- Interval-classes and subclasses emerge in diatonic heptatonic and pentatonic modes, enabling the development of multi-part forms of music and theory of composition.

The hypothesis of gradual increase in complexity of music by means of adding up more pitch classes is supported by the biologically rooted limitations in short-term memory. Its informational threshold has been established at 7+/-2 "chunks". For music processing, this means that no more than 9 elements can be combined in a meaningful compositional unit, which is a musical phrase. Systemic usage of more than 9 elements necessarily requires compression of information, achieved by "chunking" the chunks - i.e., engaging the combinatorial morphology, hierarchical organization of elements, and syntactic rules. Ditonic and tritonic music systems support music without syntax. Tetratonic music might be restrained to the elementary structures alone within separate genres and styles, but likely to exceed the elementary threshold within the entire music culture, because tetratonic music systems afford 24 melodic intonations. Hence, the borderline between music with and without syntax lies within tetratony: once the 4th pitch class is discovered and added to a music system, the latter is bound to generate hierarchical structures and music syntax.

We propose to call the timbral, ekmelic, khasmatonal and oligotonal music cultures esoteric following the terminology, adopted in regard to languages spoken by small groups that are organized in close-knit social networks (most present-day hunter/gatherer societies). This is in distinction from the exoteric music cultures, characterized by hierarchical tonal organization and centrality of syntax - in line with exoteric languages spoken by large groups that are organized as open societies.

Our model of the progressive development of tonal organization in music generally agrees with the ethnomusicological data. No ethnicity is known to use an exclusively ditonic music system. Ditonic genres and styles are encountered in tritonic and tetratonic (sometimes pentatonic) music cultures of indigenous populations of North and South America, northeastern Eurasia and Volga Plato, northern India, Sri Lanka, Australia, New Zealand, East Polynesia, and New Guinea. This suggests that the current tritonic traditions have evolved from the ditonic ones, similarly, the tetratonic traditions from the tritonic ones and so on - until they reach 7. This developmental pattern has been established for many indigenous cultures of Siberia and the Far East, most notably for Chukchi, Yakut, Nanai, Evenk, Nivkh, Orok, Khanty, Mansi, and Selkup traditions. A number of European nations (Slavic, Baltic, Turkic) that currently cultivate Western classical music also retain traces of tritonic and tetratonic traditions, conserved within specific genres (epic, religious, children). On the other hand, as of today, no evidence of systemic "simplification" of tonal 
organization has been revealed in the analysis of music and compositional practices of folk prediatonic music cultures. Well-documented examples of the "regressive" complexity are the abandonment of heptatonic diatony by Chinese traditional music in favor of pentatonic diatony sometime during 6,600-200 BCE, completed in the Middle Ages, and the extinction of the enharmonic and chromatic genera of Ancient Greek music that left only diatonic genus functional by the 3rd century AD. But both examples relate to quite complex diatonic and very complex chromatic music systems. Nothing similarly regressive is known for simpler forms of tonal organization.

It looks like the primary factor that makes the progressive development of pre-diatonic stages onedirectional is the co-evolutionary growth of complexity in social organization: from parent/child to family, from family to kin, from kin to tribe, and thereafter to tribal coalition. This evolutionary development is fueled by the reduction in reactive aggression within a social network. Family and kin-related structures are exceedingly stable and resilient to pressures (usually damageable only through the destruction of the entire structure). The first historic socio-political structure that is characterized by fragility is the city-state (whose structure corresponds to the diatonic hierarchy) - it can be damaged by internal or external upheavals and lose the obtained complexity of organization.

Family- and kin-related structures seem to directly influence the numerosity and functionality of pitch-classes in a musical mode through the process of acquisition of music skills in childhood, accompanied with progressive broadening of the scope of social interaction and developing attachments to a wider circle of people. Yet another possible connection between social structures and schemes of tonal organization comes from the practice of family and community performance, so common in pre-industrial societies. Thus, duetting with one's mother might support the model of the auxiliary ditonic mode, "trioing" with both parents - the auxiliary tritonic mode, etc.. What makes such structures non-regressible is the incorporation of every simpler scheme into the more complex one while retaining the functionality of the incorporated scheme. Thus, the formation of triangular relations between the child, mother, and father in no way cancels the previously formed dyadic mother-child relations. Similarly, tritonic modes absorb the functionality of ditonic modes, and emmelic modes of the ekmelic modes. The same correspondence exists in the numerosity of the performance settings: e.g., rules for trio performance incorporate rules of duet performance, rules for quartet - rules of trio, etc..

The threshold of this inclusiveness seems to lie somewhere between the notions of "chamber" and "orchestral" music. Orchestral setting requires a special arrangement of music, different from chamber, and is more demanding than chamber music. Noteworthy, the orchestral music was brought to life by the Bronze Age states. Correspondingly, the latter were very much prone to disintegration - evident in the cross-cultural wave of the Late Bronze Age Collapse that rolled over entire Eurasia. Potential instability of the state under external and internal political pressures (in contrast to great stability of clan-organized communities) finds its correspondence in relative volatility of music systems, instituted through statehood. Such systems are characterized by constant changes, including upgrades and downgrades of certain genres, styles, techniques, and theories. However, the downgrades of such music systems are brickwall-limited to the diatonic framework - there is no evidence of an advanced chromatic, microchromatic, hemiolic, hypermodal, or diatonic music system regressing to a simpler non-diatonic hexatonic, pentatonic, tetratonic, etc., music system. The emergence of interval-based processing of pitch seems to hold the diatonic foundation firmly in place, once it has been established. Even century-long conquests 
of states that cultivated diatony by nomadic conquerors with pre-diatonic music traditions (e.g., Mongol rule in China, Persia, Georgia, and Armenia) did not revert music cultures of conquered states to pre-diatonic stages. This clear borderline separates the diatony from all simpler schemes of tonal organization.

Based on the totality of the arguments presented above, we propose a new model of co-evolution of music and language under the effects of the evolutionary changes in aggression management. This model provides a rationale for the changes in the cultural history of our species which underlie the genesis and development of languages and music. We are convinced that the evolution of music systems and languages goes in parallel, and they can be conceived as two different products of the same biological/cultural process, determined by the increased feedback loop between the reduction of reactive aggression and the sophistication of languages and music.

We adopt the musilanguage as the starting point for the hominin communication's breakaway from typical forms of vocal communication amongst non-human primates and identify 4 stages to follow musilanguage. We do not view each stage as a clear-cut "monolithic" order of things. As environmental and social conditions are always in the process of change, we can confidently assume that the cultural features, characteristic of each stage (including language types and musical types), were also variable within a particular stage. Also, we should expect some variations in the chronology of the geographical distribution of our stages. It would be unrealistic to expect that each stage started at the same time worldwide. This view is in line with current evidence of modern human behavior appearing in different regions at different points of time. It is beyond the scope of this paper (and beyond our expertise) to review the evidence of the nature and timing of changes in human behavior and culture in different world regions throughout history. We focus on the continent of Eurasia, since we believe that the paleoecological changes there were the most conducive to the genesis of music and language, and define our stages based on the data related solely to our species (mentioning other hominins only in reference to the timeline of cultivation of musilanguage and proto-music/language, as well as the emergence of kinship). However, we reserve the possibility that other close hominins, particularly, Neanderthals and Denisovans, will fit in the first stages of our model, particularly if evidence of their management of reactive aggression emerges.

\section{Stage 0: musilanguage - before 300 kya (see 7.1)}

This pre-human stage probably did not essentially differ from animal communication, relying on the single-signal "monologic" display of the signaler's affective state and the deictic reference to something observable to the signaler. The likely distinction of musilanguage from animal calls was the presence of conventional acoustic forms of expression, reserved for specific applications, such as emitting loud collective signals to fend off dangerous predators and individual grunting patterns to accompany caretaking activities. The latter might have featured a repertory of relatively well-structured signals distinguishing different types of caretaking. The entire genus Homo is characterized by longer altriciality and short interbirth intervals, which certainly promoted elaboration and specialization of communication patterns engaged in childcare of multiple children. As such children aged, they were likely to keep using the acquired expression patterns, perhaps also in non-children-related activities, passing the established repertories of expression to the next generations. We mark the end of musilanguage by the time of the emergence of Homo sapiens, since the latter was endowed with cognitive innovations necessary for transcending the musilanguage stage [601]. Because of the lack of reliable information on social life of earlier 
hominins, we chose the tentative date of 300 kya to terminate this stage until more information on prosociality of human ancestors becomes available.

\section{Stage 1: proto-music - 300-200 kya (see 7.2)}

Stage-1 starts with the emergence of our species, roughly 300 kya. However, since the levels of reactive aggression were still high, this stage might relate to earlier hominins, particularly, Neanderthals (and, perhaps, Denisovans), who shared with humans the basic capacity to sing and possessed culture, including some signs of symbolic behavior [438,496,602]. Discovery of singing and development of a taste for it would constitute the clear cut-off point to distinguish proto-music from musilanguage - as well as from proto-language. Another likely source for emergence of proto-music would be the accidental discovery of rhythmic knapping during collective manufacturing of stone tools. Naturally entrained, knapping would obtain a metrically regular rhythm that could acquire specific semantic values and function as a musical sign to convey emotional information through the ritual use.

Aggressive and caretaking applications of musilanguage might have also gone through ritualization and turned into proto-musical genres to promote the development of individualized variants of stereotypical expressions, enriching the proto-musical lexicon. The emergence of motherese very well could be the outcome of such ritualization. Yet another sphere of crystallization of patterns of tonal organization could be the nearly universal developmental phenomenon of musical babbling (it typically occurs during the second year of life). Similar ludic activities of playing with one's mouth and respiratory apparatus is a highly probable candidate for forging proto-music. Motherese and musical babbling together very well might have supported the continuity of proto-musical tradition, necessary to transform it into music. However, this assumption should not concern Neanderthals, because we can apply the paradigms (like motherese and babbling), extracted from modern human societies only to humans of the past and not to the other hominins.

Because reactive aggression was still high, the formation of proto-linguistic patterns of communication must have followed the interpersonal bonds, established by proto-musical communication. Angry disposition must have prevented patient and cooperative turn-taking and listening to long utterances, limiting communication to single-word commands, threats, and exclamations. We expect the words referring to hunting, gathering, and especially, parent/child relations to have directed verbal communication away from music-like one, the more so as sustenance technologies and kinship relationships continued to evolve to more complex forms.

\section{Stage 2: timbre-oriented music - 200-110 kya (see 7.3)}

This stage is mostly human, although, as noted, we cannot rule out the possibility that other hominins, especially Neanderthals - the people of the North - generated some rough forms of this type of music. Timbre-based distinctions between different registers and types of modulations, such as observed in animal vocalizations and cries of human infants, constitute the most likely domain for transformation of proto-music into music. The deciding factor here must be the transition from the instinctive use of expressive means, determined by biologically ingrained reflexes and specific to certain types of stimuli, to the deliberate use of conventional means of auditory expression, capable of violating the biological encoding schemes. A milestone in this direction would be the genesis of timbral musical modes - i.e., a set of timbre-classes joined 
together according to the performer's idea of a good match, most suitable for expressing a specific semantic content. The model for tone-matching could come from social interaction, at first, between mother and child, and thereafter, between other closely related persons - both communicating parties are known to intuitively imitate the expressive attributes of each other's vocalizations. The first timbral modes might have combined those timbre-classes that were consistently engaged between a specific mother-child pair. Musical intonations made of such timbre-classes could be thereafter adopted by a wider community through the practice of childcare of multiple children.

Similarly, pre-linguistic communication might have evolved from proto-linguistic one, following the course similar to music - euphonically coupling the already established "words" (as it is observed in the use of reduplicatives in the earliest stages of acquisition of speech). Single-word utterances could be combined in a pair-wise fashion, prototyping rudimentary two-slot grammars that regulated the use of compounds, either proto-nouns or proto-verbs, to characterize objects, persons, and events. That said, we find it plausible that these early grammars were heavily used for creating colorful derogatory expressions. This would have provided a resource for replacing physical aggression with less costly verbal aggression, thereby reducing the levels of reactive aggression. The tendency to integrate words was probably brought to life by a common underlying mechanism (i.e., cortico-subcortical networks), supporting inhibition of aggression and language computation - two core dimensions of language evolution. The same tendency must have been at work in the music domain, forging the basic forms of joining anthropophonic and naturophonic (see 7.5) intonations together, eventually producing timbral modes.

Semiotically, music and language both were initially determined by the physical convenience of articulating sounds (anthropophonic intonation), thereafter exploring the onomatopoeic expression (naturophonic intonation). However, the need to effectively convey an emotional state probably pushed music towards longer exposures of the same subject of expression and reliance on vowellike articulations to support singing out, thereby promoting the integration of sounds into a long musical phrase through matching mutual acoustic attributes (timbral mode). Language, on the contrary, probably went in the direction of increasing phonemic contrasts and reliance on consonant-like articulations, emphasizing the margins of words to facilitate their accurate recognition, while conveying less of emotional information and more of propositional content. Both tendencies moved music and language away from onomatopoeic semiosis to greater abstraction of expressive means. The usage of such patterns that bore no or little resemblance to environmental sounds, objects, and events would have required the knowledge of conventions and, therefore, in itself presented a binding force to forge user-communities.

We assign a crucial role in the consolidation of social groups to the paleoenvironment. Frequent alternation of cooling and warming as well as arid and pluvial periods certainly caused significant fluctuations in group sizes. The depopulation periods set the conditions for the increase in codependence of group members, strengthening their bonds and stimulating personal and interpersonal uses of music. Furthermore, the dangers of incest posed the necessity to introduce some form of marking an individual and their posterity - which was best satisfied by something similar to personal song. During the periods of demographic growth, patterns of communication established during the depopulation periods had a better chance of survival, spread over larger territories and passed on to a greater number of people. 
Stage-2 might have spanned between roughly 200 and $110 \mathrm{kya}$, a time period corresponding to the long Riss Glaciation and the subsequent Riss-Würm Interglacial period. Eurasia seems to make a more likely place for the emergence of the tradition of tonally organized "private music" because of its harsher climatic transitions, emergence of species adapted for life in cold north, and the evidence of interbreeding between different genera of homo. The flow of genes in all directions manifests the presence of conspecific sympathy between different populations, suggesting the existence of at least some basic notion of kin and ancestral lineage. After all, the hybrid posterity definitely looked different from the rest of the tribe, yet was evidently cared for (otherwise, the differently-looking individuals would not have been able to survive on their own and pass their genome on).

\section{Stage 3: the genesis of pitch orientation - 110-10 kya (see 7.4)}

This stage might have started by $110 \mathrm{kya}$, when the first evidence of behavioral modernity appeared in many parts of the world, especially in Europe. In our view, the low levels of reactive aggression, achieved at the peak of this stage, around $50 \mathrm{kya}$, should have further increased the frequency and diversity of contacts between people, at least within a kin. Since increased prosociality results in an extended juvenile period, one could expect an extended period of learning and socializing for children, with more opportunities to acquire musical and linguistic features from adults and through play. Overall, these changes favored the emergence of more sophisticated grammar (e.g., the first hierarchical grammars expressing transitivity), triggering an accelerated feedback loop between the early forms of grammar and growing conspecific sympathy. This was likely to support the ongoing complexification of grammars, in turn, contributing to further reduction of reactive aggression.

The most confident proxy of the languages spoken during Stage-3 are the esoteric languages of the present-day hunter-gatherer societies, living in small isolated close-knit communities with high proportions of native speakers. Such languages typically exhibit larger sound inventories and complex phonotactics, opaque morphologies (with more irregularities and morpho-phonological constraints), limited semantic transparency (with abundance of idioms and idiosyncratic speech), reduced compositionality, and unsophisticated syntactic devices, with reduced embedding and frequent parataxis.

For music, the primary achievement towards the end of this stage was the emergence of crosscultural pitch orientation. The evidence for this comes from over hundred bone pipes (aka flutes) uncovered in caves (often 3 pipes per cave) across a wide region from South Germany to Spain, dated 36-30 kya. The typological similarities in their construction suggest the ongoing cultural interaction along the Danube Corridor as part of a pan-European cultural network that existed ca 45-30 kya. Such clustering of musical instruments designed to generate multiple (at least 3) pitch classes, confined to Europe and separated by 35-20 thousands years from the earliest bone flutes in East Asia, suggests that the genetic divergence of Western and Eastern Eurasians, dated by ca 40 kya, must have been accompanied by the divergence of tonal organization of music. This conclusion is further supported by the fact that all surviving indigenous timbre-oriented traditions characteristic of the music culture of an entire ethnos (e.g., Nenets, Nganasan) are located in northeastern Eurasia. Jaw Harp, world's most popular timbre-oriented instrument, probably originated around the Amur region, spread all over Asia, and was imported to Europe during the late Middle Ages. Jaw Harp seems to represent the organological model of prehistoric East Eurasia versus bone pipe that represents the pitch-oriented music of West Eurasia. 
Pitch orientation most likely emerged as a result of the interaction of at least 3 factors. Between approximately 110-10 kya, caves became a common living place, especially in Europe and Western Asia. Caves with fire (the habitual use of which was introduced in Europe about 300 kya) provided an ideal shelter and environment for cultivating collective forms of music. Listening to musical sounds in dark labyrinths with strong reverberation surely distorted the timbral characteristics of familiar sounds due to the comb-filtering reflections from the walls, while emphasizing the resonant harmonic spectrum and extending the decaying tails of sounds, so that every change of pitch level generated a harmonic dyad (the overlap between the echo of the previous pitch and the onset of the new pitch). As a result, pitch changes became more salient, attracting attention to the fundamental frequency and causing timbral melodies to be tracked in their pitch aspect. This, in effect, converted timbral modes into pitch modes. Conversion was facilitated by the use of reference pitch, provided by bone flutes and stalactites tuned to specific pitch (e.g., the Paleolithic Nerja scale). The oldest bone flutes (Aurignacian) were uncovered in caves of Southern Germany. Reference pitch constituted the second factor for the rise of pitch orientation. Many megalithic sites resonate at the frequency of $110 \mathrm{~Hz}$, which is the average frequency of a male voice, suggesting that these caves were selected for their "musicality". Socalled "singing caves" are known in Tuva (Altai), where the local indigenous population cultivates timbre-based deep-throat singing. One of its styles is called "cave rasping." Such resonanceoriented singing as well as timbre-oriented singing to a reference pitch forged a new type of musical intonation - organophonic (see 7.5).

The third factor was probably the intuitive "pitch correction" amongst the partakers in collective singing of the same melody. They instinctively avoid mismatches in the order of about 25 cents and voluntarily correct mismatches about 200 cents, ending up by singing in perfect harmonic intervals (most commonly, unison and octave, more seldom 5th or/and 4th). Collective monophonic singing tends to spontaneously negotiate the intonation standard shared by the majority of participants. On the other hand, long chain transmission of indefinite-pitch melodies was experimentally shown to induce the discretization of pitch values. The same tendency affects rhythmic organization, causing quantization of duration values. For this reason, reproduction of the same timbral melody in a sizable community (provided that pitch changes are salient and noticed) is likely to replace unquantifiable timbre-classes with quantifiable pitch-classes and put in place a new form of categorization of melodies, based on the distinction between melodic leap and step. Once established, this categorization is likely to lead to the recognition of distinctions between normative, small, and large steps and leaps. The metapopulation of Europe started growing from $17 \mathrm{kya}$ on, which sets the likely time frame for the onset of the tendency to discretize pitch-oriented melodies into pitch-classes according to the discrimination of sizes of steps and leaps.

\section{Stage 4: collective music - 10 kya-present (see 7.6)}

With the advent of the Holocene, the steady demographic growth promoted inter-group contacts and extensive social networks (trade and intermarriage), increasing the need in exchanging decontextualized meanings and in know-hows with strangers. This favored the emergence of exoteric languages. The latter are known to expand vocabularies, increase syntactic complexity, introduce compositionality, and enhance semantic transparency. By contrast, their phonology exhibits simpler sound combinations and more regular morphologies as compared to esoteric languages. Similar traits characterize the earliest documented music theories of antiquity: in comparison to traditional folk music, they standardize intervallic typology and tuning, while prescribing rules for combining pitch-classes, which reduces the variety of musical modes, musical intonations, and the 
number of possible inflections of pitch-classes. Such standardization usually occurs via political reforms, administered by the government or religious authorities - in sharp contrast to the previous state of music organization. Rationalization of rules and uniformity of pitch- and interval-classes promote the use of larger music ensembles, invention of more musical instruments, development of their performance techniques, creation of multi-part music, and genesis of cyclic music forms with contrasting sections. All of these leads to the exponential growth in complexity of tonal organization, posing the need in music education and theory of music composition. Formal knowledge of rules of using music and language becomes the criterion of distinguishing cultural "outsiders" from the "insiders" ("us" versus "them").

We have hypothesized that the advance of exoteric-like languages and music systems can be linked to the revitalization of proactive aggression. The latter exists amongst other primates (notably chimpanzees), while being absent in those few that have gone through the reduction of reactive aggression, like bonobos. In contrast to chimpanzees and bonobos, present-day humans combine low reactive aggression with relatively high proactive aggression. Our contention is that the growth of proactively aggressive behaviors might have been facilitated by the advent of exoteric-like music and languages. The latter exceed esoteric-like languages in capacity to support conscious planning and knowledge-sharing with other members of the same complex society, thereby promoting the emergence of cultural institutions relevant to warfare and enmity towards a singledout party. Music's capacity to consolidate a social group then becomes a political weapon.

Instrumental for this development is the transformation of organophonic intonation into metrophonic. The earliest organophonic intonations came from phono-instruments, such as the arrow, and from the "accidentally discovered" instruments, like buzzers and whizzers - all characterized by indefinite pitch. Invention of reeds and whistles made pitch more defined. More sophisticated instruments, like flutes or xylophones, supported discrete well-defined pitch, compatible with a greater range of semantic expressions and more culture-specific. Organophonic intonation started supporting coordination in pitch and time, allowing multiple participants to collectively carry out complex melodic structures. However, different musical instruments considerably varied in their intervallic typology, so that the musical modes based on one organophonic model differed from those based on another organophonic model - even if both models belonged to the same musical culture. Metrophonic intonation removed this discrepancy by adhering to a single standard for defining interval-classes by means of measuring distances between the placement of holes on wind instruments and frets on string instruments. The uniformity of interval-classes secured collective performance of massive scales, up to hundreds of performers. The extent of numerosity of the ensemble performance was determined by the precision of "metric" calculations.

Basic metrophonic intonation is often intuitively inferred by instrument-makers in village cultures across Eastern Eurasia and sustained through person-to-person informal instruction. However, such implicit music theory is descriptive rather than prescriptive and is not institutionally reinforced across the variety of local styles and genres. As a result, imprecise metrophonic intonation is limited to small ensembles, often to specific instruments, and can significantly vary between different locations - even within a small country. It is only math-based formal music theory, supported by musical notation, professionalization of music performance and teaching, and some form of administration of music altogether enable metrophonic intonation to penetrate all forms of music, including vocal, and reorganize all previous types of intonation. Such a reform manifests itself through the replacement of the repertory of multitonal or diatonic modes by the 
unified standardized system of keys, defined by the standard procedure of (re-)tuning a musical instrument. Keys "lock" the precise metrophonic standards adopted by music-users within an entire community as a norm (i.e., "correct" as opposed to "erroneous" use of interval-classes). Unfortunately, musical keys and modes are often confused due to the lack of taxonomic clarity in the majority of textbooks on music theory. However, a deeper look in the historical musicological literature reveals that the system of keys, similar to that of Western classical music today, existed in Ancient Greece, Mesopotamia, Persia, India, and China.

Metrophonic modes and keys supported the cross-cultural rise of orchestral and choral music, starting from the 3rd millennium BCE, in service for the state, in temples, palaces and outdoor public festivities. Massive performances gathered mass audiences that mixed societal strata. Ancient Mediterranean societies, especially Ancient Greek, were characterized by wide engagement of the general public in musical activities. Ancient Greeks enjoyed public music education and tutorship, participated in choral and solo competitions that were regularly held in major cities, as well as in religious festivals, and were expected to improvise songs at private parties. Standardized music became the tool of political consolidation of citizens of ancient states across kins, clans, and casts, forging a national identity along the lines of opposing the selfproclaimed "civilized" values to supposedly "uncivilized" neighboring cultures. The presence of uniformed metrophonic intonation acted as a divider and most obvious auditory identifier of "us versus them".

Structural features of melopoeia - the "science" of making good music (audible to all citizens wellversed in music) - were semantically supported by the doctrine of ethos. The sound of a specific musical mode/key was believed to trigger a certain affective state in those who heard it - so that frequent habitual listening supposedly could change one's personality type to that projected by a mode/key. The discourse on social pros and cons of certain modes occupied an important place in ancient communities. Scholars, from Plato and Aristotle to Ptolemy, left thorough descriptions of structural differences and ethic qualia, ascribed to each of the common modes. Modes were named after ethnicities that supposedly cultivated or favored them, where positive constructive traits were reserved for Greek tribes (Dorian and Ionian), whereas negative, potentially destructive traits were identified as foreign (Phrygian and Lydian). Obvious nationalistic bias consolidated Greeks against the foreign metrophonic traditions. Even greater resentment was documented in relation to the non-metrophonic music of the nomadic neighbors. Similar antithesis of civilized "us" against barbaric "them", distinguished by the "appropriate" arrangement of music and corresponding ethic concepts synonymous to Greek "ethos", was present in a number of countries of ancient Near and Far East, as well as in India.

Metrophonic music cultures were instrumental in supporting and promoting proactive aggression, directing it toward the prospective negative influence of outsiders. This marriage of metrophony with ethos proved to be amazingly long-living. Thus, during the Middle Ages, under the Christian influence, the system of musical modes and their ethic qualia became transformed, yet retained its fundamental property of consolidating congregations over the supposed "virtuous" forms of music versus "harmful" forms. Music structures and genres constituted very much the issue of suppression throughout the history of Christianity: e.g., repudiation of paganism (Fathers of the Church against chromatic and enharmonic music of the antiquity), prosecution of heresy (e.g., Spanish inquisition's stand against Jewish, Gypsy, and Moorish musical influences), religious wars (e.g., the crusade against mannerist polyphony patronized by the Avignon Papacy during Western Schism), and anti-hedonistic censorship (e.g., Puritan prosecution of operatic and organ 
forms of music). The flourishment of nationalistic music throughout the 18-19th centuries all-over Europe that spread into other continents should be regarded as the revival of the union of ethos and metrophonic music, set to glorify the traits of one nation and vilify those of another nation in the most audible way. The 20th century practice of government's use of music as a propaganda tool in cultural warfare (e.g., Western avant-garde versus Communist "people's music") also grows from the same foundation. For this reason, we time the 4th stage as continuing until the present.

\section{Testing the model}

Although hypotheses about the origins and evolution of music are difficult to verify, since they are based on indirect evidence from diverse sources, we expect our model to be testable, because it generates specific predictions in specific domains that can be subjected to empirical inquiry.

One important domain of verification is that of disorders. Cognitive conditions that affect the ability to socialize differ in their influence on aggressive behavior. Also, low levels of reactive aggression affect the manifestation of physical and behavioral traits. Thus, such traits are found attenuated in autism spectrum disorder (ASD) which features high levels of reactive aggression [603]. Conditions like Williams Syndrome (WS) [604], in contrast, are characterized by friendly, prosocial behavior. Interestingly, Masataka [605] has shown that young people with ASD display more interest in dissonant music than neurotypical controls. By contrast, people with WS, known for their marked interest in music, often fail to properly identify interval violations [606] or pitch differences between two contiguous tones [607]. Such people seem to be more sensitive to the global patterns of music, particularly, to the emotional aspects of music, manifesting heightened auditory sensitivities, a propensity towards musical activities, and marked responses to expressivity (see [608] for review). At the same time, despite their different, at times opposite socialization profiles, ASD and WS show similar memory advantages for vocalise [609].

Another putative arena for testing some of our predictions is Tourette's syndrome [367,369]. This condition is typically associated with high levels of reactive aggression, which often materializes as tics and profane language. Conditions like Tourette's syndrome might provide a window into earlier stages of human evolution under the effect of changes in aggression management and particularly, Stage-2 in our model. Noteworthy, patients display differences in brain activity during music processing [610], whereas musical intervention (in the form of music performance, listening to music, or mental imagery of music) reduces the severity of the symptoms [611]. Overall, we are interested in knowing more about musical abilities by people affected by these and similar disorders.

A second important domain is neurobiology. The basic backbone of our proposal that a reduction in reactive aggression might have contributed to the complexity of language and music relies on the fact that the same cortical-subcortical circuits inhibit aggression and control syntactic chunking. The reduction of aggression and the emergence of early expressive forms of language aimed for verbal aggression might reinforce this mechanism [367]. We hypothesize that the same virtuous feedback loop could be involved in the evolution of music, where music genres, dedicated to expression of the musical emotion of anger, have occupied an important place from the earliest known civilizations to the present. Accordingly, fMRI studies aimed to investigate the contribution of aggression-specific brain circuits to music processing and the influence of changes in aggression levels on music processing will be very welcome. 
A third promising direction is comparative ethology/cognition. In animals, differential sensitivity to specific aspects of music have been shown to depend on stress conditions. For instance, in dogs, low-pitch musical tracks increase alertness and ultimately, stress levels [612]. Likewise, birdsongs significantly influence the behavior of conspecific listeners, often promoting social bonding or aggression in a way similar to music. Rothenberg and colleagues [613] have suggested that this effect might be driven by principles, similar to those that make music effective in inducing emotional responses in humans. Because of the analogies between the involved brain circuits, animal models might be useful for studying the effects of reduced aggression levels on human musical abilities. Particularly promising is the comparison of wild and domesticated varieties of animals.

A fourth domain where our conclusions can be evaluated and corrected is cultural anthropology. Of particular interest for our hypothesis would be mapping musical diversity onto the esotericexoteric continuum of human societies, for which some information is available (e.g., the Standard Cross-Cultural Sample). Knowing more about the relationships between exo/esotericity and musical features should enable one to make more robust inferences about the features of music in the past. Additional support to our hypothesis would come from the discovery of correlations between the dominant types of music performed in present-day human societies (either esoteric or exoteric) and physiological and behavioral manifestations of changes in the aggression levels. If reduced reactive aggression and increased prosociality (or conspecific sympathy) generally correlate with such traits as reduced sexual dimorphism [288], we would predict that these traits are heightened in human groups cultivating exoteric types of music.

An interesting direction for research could be the comparative study of languages, music and aggression levels in those traditional societies that retain a hunter/gathering lifestyle without the use of domesticated animals (e.g., Australian Aborigines) as opposed to those that heavily relied on animal husbandry from ancient times (e.g., Turkic peoples of Siberia and Middle Asia). According to our model, members of esoteric language/music communities should exhibit more marked features of conspecific sympathy as compared to exoteric language/music users, and such communities without the history of animal domestication are likely to feature higher levels of reactive aggression. Eventually, as candidates for mammal domestication seem to have been positively selected recently in Europe [291], one could expect as well some genetic differences between esoteric and exoteric communities. But this idea needs careful examination.

An important place in our model occupies the cross-cultural phenomenon of personal song. Many of our claims can be verified by establishing the exact geographic distribution of this tradition and its affiliation with specific genetic markers. Its connection with the ancient Western Eurasian and northeast Asian human populations that we draw, with its possible Neanderthal origins, might be proved or disproved by the geomusicological and genetic correlations. Perhaps, the most intriguing question is whether personal song exists in Africa. Our conclusions about the place of personal song in the evolution of human musicking from animal communication can be verified by experimental studies of perception of personal songs by indigenous populations habitual to its use - especially, their ability to recognize and interpret the morphological changes within a specific song.

Experimental psychoacoustic approach can verify our characterization of the poorly known types of music: timbre-oriented, ekmelic, khasmatonal, and oligotonal. Most information about their 
perception comes from the ethnomusicological sources, anecdotal accounts, and extrapolations of the findings from the lab experiments of perception of isolated acoustic attributes, mostly of the Western classical music. Such findings can be very far from the real-life perception of nonWestern forms of music by indigenous populations. Invaluable for our understanding of prediatonic forms of music would be the empirical study of their perception by those subjects for whom such music is native and who maintains traditional lifestyle in relative isolation from the Western influences. An example of this approach is the study of harmonic hearing by McDermott and colleagues, conducted as a field-research in the Amazonian region in Bolivia [614]. Even more reliable is the method of verifying the analysis of music structures by means of generating a sample of music according to the inferred principles of tonal organization and playing it back to indigenous users of the analyzed music for their validation of the sample's "authenticity" - pioneered by Simha Arom in Central Africa [615]. Yet another promising direction is the computer-based statistical analysis of the distribution of specific traits of intervallic typology in large databases of recordings of indigenous music - coupled with the cross-comparison of the geographic, linguistic, instrumentation, and genre meta-data [616].

The sixth prospective venue of research is the experimental studies of structural changes in chain transmission of music across specific groups of music-users, as implemented by Lumaca and Baggio - controlling for the semantic aspect of music stimuli [139,157]. This would parallel the rich research on language transmission in experiments involving artificial languages and iterated learning. Patterns of timbral, ekmelic and oligotonal music can be investigated in the same way as the transmission of discrete pitch structures. Equally important would be to compare the longchain transmission across large groups of actors between music and language. The existing experimental studies of invented languages for practical reasons keep the number of participants and generations small. It would be very interesting to see what would happen in an experiment involving hundreds of subjects and extending over weeks. Also, the tree and inverted tree transmission formats should be experimentally tested in comparison to the person-to-person and self-communication.

Finally, it might be worth exploring the approach proposed by Vygotsky and Luria in the late 1920s. In 1929 they launched a large-scale longitudinal study of verbal and musical abilities of 150 pairs of mono- and dizygotic twins, 6-14 years old, in an attempt to differentiate the impact of genetic and cultural factors on ontogenetic development [617]. The coefficient of verbal abilities was found 0.89 for dizygotic twins versus 0.96 for monozygotic twins, whereas the coefficient of musical abilities, respectively, 0.67 versus 0.93 . Vygotsky argued that since the most substantial developmental difference between early age mono- and dizygotic twins was their genetic inheritance, the difference in rating a specific psychological function between the mono- and dizygotic twins would express the share of genetic contribution to it [115]. The nearly quadruple advantage of the delta for musical skills over that of verbal skills, according to Vygotsky, indicated the greater hereditary determination for music. Similar approach could be applied to measuring levels of aggression and friendliness and establishing their correspondence to specific verbal and musical skills.

\section{REFERENCES}


[2] Brown S. The "Musilanguage" Model of Language Evolution. In: Brown S, Merker B, Wallin NL, editors. Orig. Music, Cambridge, MA: MIT Press; 2000, p. 271-300. https://doi.org/10.1037/e533412004-001.

[3] Vygotsky LS. The collected works of L.S. Vygotsky. Child Psychology. vol. 5. New York: Plenum Press; 1987.

[4] van der Veer R, Valsiner J. Understanding Vygotsky: A quest for synthesis. Hoboken, NJ: Blackwell Publishing; 1991.

[5] Mazel L. On certain aspects of Asafyev's concept [О некоторых сторонах концепции Б.В. Асафьева]. In: Prudnikova I, editor. Essays Theory Anal. Music Статьи По Теории И Анализу Музыки, Moscow: Soviet Composer; 1982, p. 277-307.

[6] Tull JR, Asafyev B. B.V. Asaf'ev's Musical form as a process: translation and commentary. vol. 3 volumes. Ann Arbor, Mich.: University Microfilms International [Publisher]; 2000. https://doi.org/610363518.

[7] Asafyev B. Selected Works [Избранные труды]. vol. 1. Moscow: Academy of Science of the USSR; 1952.

[8] Zemtsovsky I. Asafyev and methodological foundations of intonational analysis of the folk music [Б.В.Асафьев и методологические основы интонационного анализа народной музыки]. In: Kolovskii OP, editor. Crit. Musicol. Критика И Музыкознание, vol. 2, Leningrad: Muzyka; 1980, p. 184-98.

[9] Mehr SA, Krasnow MM, Bryant GA, Hagen EH. Origins of music in credible signaling. Behav Brain Sci 2020:1-41. https://doi.org/10/ghj9f3.

[10] Savage PE, Loui P, Tarr B, Schachner A, Glowacki L, Mithen S, et al. Music as a coevolved system for social bonding. Behav Brain Sci 2020:1-36. https://doi.org/10/ghhxnd.

[11] List G. Speech melody and song melody in Central Thailand. Ethnomusicology 1961;5:16-32. https://doi.org/10/fhj755.

[12] Nikolsky A, Alekseyev EYe, Alekseev IYe, Dyakonova VE. The overlooked tradition of 'personal music' and its place in the evolution of music. Front Psychol 2020;10:3051. https://doi.org/10/gmnfww.

[13] Nikolsky A. The pastoral origin of semiotically functional tonal organization of music. Front Psychol 2020;11:1358. https://doi.org/10/gmnfx2.

[14] Masataka N. Music, evolution and language. Dev Sci 2007;10:35-9. https://doi.org/10/fjks68.

[15] Ravignani A, Thompson B, Filippi P. The evolution of musicality: What can be learned from language evolution research? Front Neurosci 2018;12:20. https://doi.org/10/gc2pbk.

[16] Nikolsky A. Non-communicative Music Grammars of the 20th Century. Atlanta, Georgia: ATLOSCon; 2019. https://doi.org/10.6084/m9.figshare.14850543.

[17] Nazaikinsky YV. Style and genre in music [Стиль и жанр в музыке]. Tbilisi, Georgia: Tbilisi State Conservatoire; 2013.

[18] Nikolsky A. A new method of modal multifactorial analysis of tonal organization in music. Front Psychol 2020;11. https://doi.org/10/gmnfzv.

[19] Mertens P. The Prosogram: Semi-Automatic Transcription of Prosody Based on a Tonal Perception Model. Proc. 2nd Int. Conf. Speech Prosody, 2004, p. 549-52.

[20] Davis RF. Ma'lüf: Reflections on the Arab Andalusian Music of Tunisia. Lanham, MD: Scarecrow Press; 2004.

[21] Espy WR. The Garden of Eloquence: A Rhetorical Bestiary. New York, NY: Harper \& Row; 1983.

[22] Margulis EH. On Repeat: How Music Plays the Mind. Oxford, New York: OUP USA; 2014.

[23] Sheikin YI, Dobzhanskaya OE, Ignatyeva TI, Nikiforova VS. Sounding landscapes in the intonational acoustic cultures of indigenous peoples of the Arctic: the formulation of a problem and methodological approaches [Звучащие ландшафты в интонационно-акустической культуре коренных народов Арктики: постановка проблем. In: Dobzhanskaya OE, Ignatyeva TI, editors. Sound. Landsc. Arct. Звучащие Ландшафтты Арктики, Novosibirsk, Russia: Nauka; 2019, p. 7-34.

[24] Tagg P. Music's Meaning: A Modern Musicology for non-musos. Larchmont, N.Y.: Mass Media's Scholar's Press; 2012.

[25] Nikolsky A. The Correspondence Between Composition in Fine Arts and Music throughout History. vol. 7. 2016. https://doi.org/10.6084/m9.figshare.15081477.

[26] Mazel L. Structuring of musical works [Строение музыкальных произведений]. Moscow: Muzyka; 1979.

[27] Réti R. The Thematic Process in Music. New York, NY: Macmillan Publishers; 1951.

[28] Nikolsky A, Alekseyev EYe, Alekseev IYe, Dyakonova VE. What the "Talking Jew's Harp" is saying: Jew's harp and personal song as the foundation of timbre-oriented musical systems [О чем говорит «говорящий варган»: варган и личная песня как основание темброво-ориентированных музыкальных систем]. Lang Folk Indig Peoples Sib 2019;1:5-32. https://doi.org/10/gmnfvw.

[29] Nikolsky A. Emergence of the distinction between "verbal" and "musical" in early childhood development. In: Masataka N, editor. Orig. Lang. Revisit. Differ. Music Emergence Neurodiversity Autism, Singapore: Springer Nature; 2020, p. 139-216. https://doi.org/10.1007/978-981-15-4250-3_7.

[30] Ohala JJ, Eukel BW. Explaining the intrinsic pitch of vowels. In: Channon R, Shockey L, editors. Honor Ilse Lehiste, vol. 60, Dordrecht: Foris; 1987, p. 207-15. https://doi.org/10.1121/1.2003351.

[31] Barbour JM. Tuning and Temperament: A Historical Survey. New York: Dover Publications; 2004.

[32] Daniélou A. Music and the Power of Sound: The Influence of Tuning and Interval on Consciousness. Rochester, Vt: Inner Traditions; 1995.

[33] Barbieri P, Mangsen S. Violin intonation: a historical survey. Early Music 1991;19:69-88. https://doi.org/10/cxgscw. 
[34] Friberg A. A quantitative rule system for musical performance. Royal Institute of Technology, 1995.

[35] Duffin RW. How Equal Temperament Ruined Harmony (and Why You Should Care). New York: Norton; 2007.

[36] Steblin R. A History of Key Characteristics in the Eighteenth and Early Nineteenth Centuries. 2nd ed. Rochester, NY: University of Rochester Press; 2002.

[37] Nikolsky A. Taxonomy of Modal Music with an Example of Modal Analysis of Ekmelic Music. Front Psychol 2015;6. https://doi.org/10/gmnfzz.

[38] Strunk WO, Treitler L. Source Readings in Music History. New York: Norton; 1998.

[39] Beliayev VM. Viktor Mikhailovich Beliayev [Виктор Михайлович Беляев]. Moscow: Soviet Composer; 1990.

[40] Kholopov Y. Mode [Лад]. Encycl Music Музыкальная Энциклопедия 1976;3:130-43.

[41] Yavorskii B. The construction of musical speech. Data and notes [Строение музыкальной речи. Материалы и заметки]. vol. 1. Moscow: Aralov, G.; 1908.

[42] Bytchkov YN. On systemic nature of modal organization in music [О системном характере ладовой организации в музыке]. Gnessin Russian Academy of Music [Российская академия музыки имени Гнесиных], 1987.

[43] Blum S. Rousseau's Concept of "Sisteme musical" and the Comparative Study of Tonalities in NineteenthCentury France. J Am Musicol Soc 1985;38:349-61. https://doi.org/10/djqzq3.

[44] Tenney J. A History of Consonance and Dissonance. New York, USA: Excelsior; 1988.

[45] Messner GF. Do they sound like bells or like howling wolves? Interferential diaphony in Bistritsa: an investigation into a multi-part singing tradition in a middle-western Bulgarian village. Frankfurt Am Main, Germany: Peter Lang; 2013.

[46] Cazden N. The Definition of Consonance and Dissonance. Int Rev Aesthet Sociol Music 1980;11:123-68. https://doi.org/10/ccqc4r.

[47] Patel AD. Music, biological evolution, and the brain. In: Levander C, Henry C, editors. Emerg. Discip. Shap. New Fields Sch. Inq. Humanit., Houston, TX: Rice University Press; 2010, p. 91-144.

[48] Loui P, Patterson S, Sachs ME, Leung Y, Zeng T, Przysinda E. White matter correlates of musical Anhedonia: Implications for evolution of music. Front Psychol 2017;8:1664. https://doi.org/10/gb2z3w.

[49] Haiman J. Decorative morphology in Khmer. In: Williams JP, editor. Aesthet. Gramm. Sound Mean. Lang. Mainl. Southeast Asia, Cambridge: Cambridge University Press; 2013, p. 61-82. https://doi.org/10.1017/CBO9781139030489.005.

[50] Levitin D. The World in Six Songs: How the Musical Brain Created Human Nature. 2nd ed. London: Penguin Books Limited; 2019.

[51] Perlovsky L. The Cognitive Function of Music. Part II. Interdiscip Sci Rev 2014;39:162-86. https://doi.org/10/gmnfjj.

[52] Dissanayake E. Ritual and Ritualization: Musical Means of Conveying and Shaping Emotion in Humans and Other Animals. In: Brown S, Volgsten U, editors. Music Manip. Soc. Uses Soc. Control Music, Oxford; New York: Berghahn Books; 2005, p. 31-56.

[53] Nikolsky A. The commonalities between melodic line, geometric line, and environmental topography in traditional cultures of Northern Siberia: "landscape aesthetics" as a model of musical genesis. Front Psychol 2016;7. https://doi.org/10/gmnfz2.

[54] Stefanija L. Functions of Music: A Survey of Research Vocabularies. Muzikos Funkc Tyrimų Terminol Apžv Lith 2007;7:6-17.

[55] Savage PE, Brown S, Sakai E, Currie TE. Statistical universals reveal the structures and functions of human music. Proc Natl Acad Sci U S A 2015;112:8987-92. https://doi.org/10/f7j74k.

[56] Trevarthen C. Human biochronology: on the source and functions of 'musicality'. In: Haas R, Brandes V, editors. Music Works Contrib. Biol. Neurophysiol. Psychol. Sociol. Med. Musicol., Vienna, New York: Springer; 2009, p. 221-65.

[57] Brown S. "How does music work?" toward a pragmatics of musical communication. In: Volgsten U, Brown S, editors. Music Manip. Soc. Uses Soc. Control Music, New York, NY: Berghahn Books; 2005, p. 1-27.

[58] Schäfer T, Sedlmeier P. From the functions of music to music preference. Psychol Music 2009;37:279-300. https://doi.org/10/ch3pkt.

[59] Hagen EH, Bryant GA. Music and dance as a coalition signaling system. Hum Nat 2003;14:21-51. https://doi.org/10/drnd8k.

[60] Schäfer T, Tipandjan A, Sedlmeier P. The functions of music and their relationship to music preference in India and Germany. Int J Psychol 2012;47:370-80. https://doi.org/10/gms2vc.

[61] van Goethem A, Sloboda J. The functions of music for affect regulation. Music Sci 2011;15:208-28. https://doi.org/10.1177/1029864911401174.

[62] Hargreaves DJ, North AC. The Functions of Music in Everyday Life: Redefining the Social in Music Psychology. Psychol Music 1999;27:71-83. https://doi.org/10.1177/0305735699271007.

[63] Schubert E. The fundamental function of music. Music Sci 2009;13:63-81. https://doi.org/10/bsjp6j.

[64] Boer D, Fischer R. Towards a holistic model of functions of music listening across cultures: A culturally decentred qualitative approach. Psychol Music 2012;40:179-200. https://doi.org/10/dxwn8v.

[65] Alekseyev EYe. Folklore in the context of modern culture: thoughts on the future of folk song [Фольклор в контексте современной культуры: рассуждения о судьбах народной песни]. Moscow: Soviet Composer; 1988. 
[66] Nettl B. The Study of Ethnomusicology: Thirty-one Issues and Concepts. Champaign, IL: University of Illinois Press; 2005.

[67] Schäfer T, Sedlmeier P, Städtler C, Huron D. The psychological functions of music listening. Front Psychol 2013;4:511. https://doi.org/10.3389/fpsyg.2013.00511.

[68] Merriam AP. The Anthropology of Music. Evanston, IL: Northwestern University Press; 1964.

[69] Zemtsovsky I. Song as a historic phenomenon [Песня как исторический феномен]. In: Gusev V, editor. Pop. Song Probl. Study Народная Песня Проблемы Изучения, vol. 6, Leningrad: State Academy of Theatrical Arts; 1983, p. 22-35.

[70] Watanabe S. Title How animals perceive music?: comparative study of discriminative and reinforcing properties of music for infrahuman animals. CARLS Ser. Adv. Study Log. Sensib., vol. 2, Tokyo: Centre for Advanced Research on Logic and Sensibility The Global Centers of Excellence Program, Keio University; 2008, p. 5-16.

[71] Howell S, Schwandt M, Fritz J, Roeder E, Nelson C. A Stereo Music System as Environmental Enrichment for Captive Chimpanzees. Lab Anim 2003;32:31-6. https://doi.org/10/fv94bt.

[72] Bowman A, Scottish Spca null, Dowell FJ, Evans NP. "Four Seasons" in an animal rescue centre; classical music reduces environmental stress in kennelled dogs. Physiol Behav 2015;143:70-82. https://doi.org/10/2h6.

[73] Huo X, Wongkwanklom M, Phonraksa T, Na-Lampang P. Effects of playing classical music on behavior of stabled horses. Vet Integr Sci 2021;19:259-67.

[74] Alworth LC, Buerkle SC. The effects of music on animal physiology, behavior and welfare. Lab Anim 2013;42:54-61. https://doi.org/10.1038/laban.162.

[75] Snowdon CT. Animal Signals, Music and Emotional Well-Being. Animals 2021;11:2670. https://doi.org/10.3390/ani11092670.

[76] Brent L, Weaver D. The physiological and behavioral effects of radio music on singly housed baboons. J Med Primatol 1996;25:370-4. https://doi.org/10/fp3wv5.

[77] Videan E, Fritz J, Howell S, Murphy J. Effects of two types and genres of music on social behavior in captive chimpanzees (Pan troglodytes). J Am Assoc Lab Anim Sci JAALAS 2007;46:66-70.

[78] Granot R. Music, Pleasure, and Social Affiliation: Hormones and Neurotransmitters. Routledge Companion Music Cogn., London: Routledge; 2017, p. 101-12.

[79] Salimpoor VN, Zatorre RJ. Neural interactions that give rise to musical pleasure. Psychol Aesthet Creat Arts 2013;7:62-75. https://doi.org/10/f4q7cj.

[80] Hefer M, Weintraub Z, Cohen V. Musical cognition at birth: a qualitative study. Early Child Dev Care 2009;179:769-83. https://doi.org/10/fdknf7.

[81] Fais L, Leibowich J, Hamadani L, Ohira L. Infant movement as a window into language processing. Gesture 2010;10:222-50. https://doi.org/10/b9rknp.

[82] Nieminen S, Istók E, Brattico E, Tervaniemi M, Huotilainen M. The development of aesthetic responses to music and their underlying neural and psychological mechanisms. Cortex 2011;47:1138-46. https://doi.org/10/cczqbc.

[83] Clark MS, Pataki SP, Carver VH. Some thoughts and findings on self-presentation of emotions in relationships. Knowl. Struct. Close Relatsh. Soc. Psychol. Approach, Hillsdale, NJ, US: Lawrence Erlbaum Associates, Inc; 1996, p. 247-74.

[84] Komiyama T, Iwama H, Osada N, Nakamura Y, Kobayashi H, Tateno Y, et al. Dopamine Receptor Genes and Evolutionary Differentiation in the Domestication of Fighting Cocks and Long-Crowing Chickens. PLOS ONE 2014;9:e101778. https://doi.org/10/f6ns3v.

[85] Sato DX, Rafati N, Ring H, Younis S, Feng C, Blanco-Aguiar JA, et al. Brain Transcriptomics of Wild and Domestic Rabbits Suggests That Changes in Dopamine Signaling and Ciliary Function Contributed to Evolution of Tameness. Genome Biol Evol 2020;12:1918-28. https://doi.org/10/gg9gxc.

[86] Chekalin E, Rubanovich A, Tatarinova TV, Kasianov A, Bender N, Chekalina M, et al. Changes in Biological Pathways During 6,000 Years of Civilization in Europe. Mol Biol Evol 2019;36:127-40. https://doi.org/10.1093/molbev/msy201.

[87] Ruan C, Zhang Z. Laboratory domestication changed the expression patterns of oxytocin and vasopressin in brains of rats and mice. Anat Sci Int 2016;91:358-70. https://doi.org/10/f8wzft.

[88] Neumann ID. Involvement of the brain oxytocin system in stress coping: interactions with the hypothalamopituitary-adrenal axis. Prog Brain Res 2002;139:147-62. https://doi.org/10.1016/s0079-6123(02)39014-9.

[89] Trut L, Oskina I, Kharlamova A. Animal evolution during domestication: the domesticated fox as a model. BioEssays News Rev Mol Cell Dev Biol 2009;31:349-60. https://doi.org/10/c5f3fw.

[90] Malloch S, Trevarthen C. Communicative musicality: exploring the basis of human companionship. New York: Oxford University Press, USA; 2009.

[91] Papoušek M. Intuitive parenting: a hidden source of musical stimulation in infancy. In: Deliège I, Sloboda J, editors. Music. Begin. Orig. Dev. Music. Competence, Oxford, UK: Oxford University Press; 1996, p. 88-112. https://doi.org/10.1093/acprof:oso/9780198523321.003.0004.

[92] Tafuri J, Villa D. Musical elements in the vocalisations of infants aged 2-8 months. Br J Music Educ 2002;19:73-88. https://doi.org/10/bgwqhk.

[93] Perlovsky L. Sapience, consciousness, and the knowledge instinct (prolegomena to a physical theory). In: 
Mayorga RV, Perlovsky LI, editors. Artif. Sapience Princ. Methods Wise Syst., London: Springer London; 2008, p. 33-60. https://doi.org/10.1007/978-1-84628-999-6_3.

[94] Berlyne DE. A Theory of Human Curiosity. Br J Psychol Gen Sect 1954;45:180-91. https://doi.org/10/bwk4rj.

[95] Kang MJ, Hsu M, Krajbich IM, Loewenstein G, McClure SM, Wang JTY, et al. The wick in the candle of learning: Epistemic curiosity activates reward circuitry and enhances memory. Psychol Sci 2009;20:963-73. https://doi.org/10/d5mngm.

[96] Dowling WJ. Cognitive Processes in the Perception of Art. Adv Psychol 1984;19:145-63. https://doi.org/10/dt9bsj.

[97] Moog H. The musical experience of the pre-school child. London: Schott Music; 1976.

[98] Holahan JM. Toward a Theory of Music Syntax: Some Observations of Music Babble in Young Children. Music Child Dev., New York, NY: Springer New York; 1987, p. 96-106. https://doi.org/10.1007/978-1-46138698-8_5.

[99] Hargreaves DJ. The development of artistic and musical competence. In: Deliège I, Sloboda J, editors. Music. Begin., Oxford: Oxford University Press; 1996, p. 145-70. https://doi.org/10.1093/acprof:oso/9780198523321.003.0006.

[100] Fitch WT. The biology and evolution of music: a comparative perspective. Cognition 2006;100:173-215. https://doi.org/10/d84vv8.

[101] Campbell PS. Songs in their heads: music and its meaning in children's lives. Oxford: Oxford University Press; 1998.

[102] Bjørkvold JR. The muse within: creativity and communication, song and play from childhood through maturity. London: Harper Collins Publishers; 1992.

[103] Barrett MS. Musical narratives: A study of a young child's identity work in and through music-making. Psychol Music 2011;39:403-23. https://doi.org/10/cs9x3f.

[104] Koops LH. "Now can I watch my video?": Exploring musical play through video sharing and social networking in an early childhood music class. Res Stud Music Educ 2012;34:15-28. https://doi.org/10/ghts9k.

[105] Burton S. An Exploration of Preschool Children's Spontaneous Songs and Chants by. Vis Res Music Educ 2002;2:1-16.

[106] Barrett MS. Meme Engineers: children as producers of musical culture. Int J Early Years Educ 2003;11:195212. https://doi.org/10/fj8gjq.

[107] Schubert E, McPherson GE. Underlying mechanisms and processes in the development of emotion perception in music. In: McPherson GE, editor. Child Music. Handb. Music. Dev., Oxford: Oxford University Press; 2015, p. 221-43.

[108] Franco F, Chew M, Swaine JS. Preschoolers' attribution of affect to music: A comparison between vocal and instrumental performance. Psychol Music 2017;45:131-49. https://doi.org/10/gmnfpd.

[109] Eerola T, Vuoskoski JK. A review of Music and Emotion Studies: Approaches, Emotion Models, and Stimuli. Music Percept 2013;30:307-40. https://doi.org/10/gmnfn9.

[110] Welch GF. Singing and vocal development. In: McPherson G, editor. Child Music. Handb. Music. Dev., New York, London: Oxford University Press; 2006, p. 311-30. https://doi.org/10.1093/acprof:oso/9780198530329.003.0016.

[111] Adachi M, Trehub SE. Children's Expression of Emotion in Song. Psychol Music 1998;26:133-53. https://doi.org/10/bgvwms.

[112] Adachi M, Trehub SE. Canadian and Japanese preschoolers' creation of happy and sad songs. Psychomusicology Music Mind Brain 2011;21:69-82. https://doi.org/10/gmnfpb.

[113] Saarikallio S. Emotional self-regulation through music in 3-8-year-old children. In: Louhivuori J, Eerola T, Saarikallio S, Himberg T, Eerola P-S, editors. Proc. 7th Trienn. Conf. Eur. Soc. Cogn. Sci. Music ESCOM, Jyväskylä, Finland: ESCOM; 2009, p. 459-62.

[114] Lewis MD, Granic I. Phases of Social-Emotional Development from Birth to School Age. Dev. Relat. Mind Brain Educ., Dordrecht: Springer Netherlands; 2010, p. 179-212. https://doi.org/10.1007/978-90-481-366678.

[115] Vygotsky LS. Lectures on Pedology [Лекции по педологии]. Izhevsk, Russia: Udmurt State University Publishing Press; 2001.

[116] Zaporozhets AV. Selected Works on Psychology [Избранные психологические труды]. vol. 1. Moscow: Pedagogika; 1985.

[117] Minks A. From Children's Song to Expressive Practices: Old and New Directions in the Ethnomusicological Study of Children. Ethnomusicology 2002;46:379-408. https://doi.org/10/drvnb3.

[118] Campbell PS. The Child-song Genre: A Comparison of Songs by and for Children. Int J Music Educ 1991;17:14-23. https://doi.org/10/bdnq5k.

[119] Loewy JV. The Musical Stages of Speech: A Developmental Model of Pre-Verbal Sound Making. Music Ther 1995;13:47-73. https://doi.org/10/gmnfn4.

[120] Vensten-Tagrina ZV. The phenomenon of personal song in traditional culture of Chukchi [Феномен личной песни в традиционной культуре чукчей]. State Russian pedagogical university named after Gertsen, 2008.

[121] Ojamaa T, Ross J. Relationship between texts and tunes in the Siberian folksongs. In: Parncutt R, Kessler A, Zimmer F, editors. CIM04 Conf. Interdiscip. Musicol., Graz, Austria: Graz University; 2004, p. 134-5.

[122] Dobzhanskaya O. The singers and Songs of the Avam Tundra: Musical Folklore of Nganasan [Певцы и 
песни авамской тундры. Музыкальный фольклор нганасан]. Norilsk, Russia: Apeks; 2014.

[123] Monelle R. The musical topic: hunt, military and pastoral. Indianapolis, IN: Indiana University Press; 2006.

[124] AudienceNet. Music Consumer Insight Report. London: International Federation of the Phonographic Industry; 2018.

[125] Clarke EF. Rhythm and Timing in Music. Psychol. Music, Cambridge, MA: Academic Press; 2007, p. 473500. https://doi.org/10.1016/b978-012213564-4/50014-7.

[126] Leedy D. Intonation. New Grove Dict Music Music 2001. https://doi.org/10.1093/gmo/9781561592630.article.53762.

[127] Hockett CF. The origin of speech. Sci Am 1960;203:89-96.

[128] Scott-Phillips T, Blythe R. Why is combinatorial communication rare in the natural world, and why is language an exception to this trend? J R Soc Interface R Soc 2013;10:20130520. https://doi.org/10/gmw7nt.

[129] Zuidema W, de Boer B. The evolution of combinatorial phonology. J Phon 2009;37:125-44. https://doi.org/10/c5xx45.

[130] Verhoef T, Kirby S, de Boer B. Emergence of combinatorial structure and economy through iterated learning with continuous acoustic signals. J Phon 2014;43:57-68. https://doi.org/10/gcpb5f.

[131] Sandler W, Meir I, Padden C, Aronoff M. The emergence of grammar: Systematic structure in a new language. Proc Natl Acad Sci 2005;102:2661-5. https://doi.org/10/bbpkzw.

[132] Dachkovsky S, Stamp R, Sandler W. Constructing Complexity in a Young Sign Language. Front Psychol 2018;9:2202. https://doi.org/10/gnt2xz.

[133] Tamariz M, Kirby S. The cultural evolution of language. Curr Opin Psychol 2016;8:37-43. https://doi.org/10/ggjk28.

[134] Kirby S, Tamariz M, Cornish H, Smith K. Compression and communication in the cultural evolution of linguistic structure. Cognition 2015;141:87-102. https://doi.org/10/f7jcnn.

[135] Smith K, Wonnacott E. Eliminating unpredictable variation through iterated learning. Cognition 2010;116:4449. https://doi.org/10/d7rxt5.

[136] Alekseyev EYe. Musical intonation in the earliest forms of folklore. The aspect of pitch [Раннефольклорное интонирование: звуковысотный аспект]. Moscow: Soviet Composer; 1986.

[137] Grauer VA. New perspectives on the Kalahari debate: A tale of two "genomes." Farming Archaeol Anthropol Hunt-Gatherers 2007;2:article 4:1-14. https://doi.org/10/gmnfjg.

[138] Sheikin YI. The history of music culture of Siberian ethnicities: a comparative historical investigation [История музыкальной культуры народов Сибири: сравнительно-историческое исследование]. Moscow: Eastern Literature, Russian Academy of Science; 2002.

[139] Lumaca M, Baggio G. Cultural Transmission and Evolution of Melodic Structures in Multi-generational Signaling Games. Artif Life 2017;20:143-62. https://doi.org/10.1162/ARTL.

[140] Nikolsky A. Opposition of folk and palace/temple music in Antiquity: on the origin of art music in light of historical ethnomusicology. Front Psychol 2016;7. https://doi.org/10/gmnfz6.

[141] Shanahan D, Albrecht J. Examining the Effect of Oral Transmission on Folksongs. Music Percept 2019;36:273-88. https://doi.org/10/gf8nkf.

[142] Mazel L. On melody [О мелодии]. Moscow: Gos Muz Izdat [State Musical Publishing]; 1952.

[143] Nikolsky A. Evolution of Tonal Organization in Music Optimizes Neural Mechanisms in Symbolic Encoding of Perceptual Reality. Part-2: Ancient to Seventeenth Century. Front Psychol 2016;7:211. https://doi.org/10/gmdd4n.

[144] Panteli M, Purwins H. A Quantitative Comparison of Chrysanthine Theory and Performance Practice of Scale Tuning, Steps, and Prominence of the Octoechos in Byzantine Chant. J New Music Res 2013;42:205-21. https://doi.org/10/ggbfrx.

[145] Boyd R, Henrich J. On Modeling Cognition and Culture: Why cultural evolution does not require replication of representations. J Cogn Cult 2002;2:87-112. https://doi.org/10.1163/156853702320281836.

[146] List G. The Reliability of Transcription. Ethnomusicology 1974;18:353-77. https://doi.org/10/bwcc4r.

[147] Alekseyev EYe. Folkloric notation. Theory and practice [Фольклористическая нотация. Теория и практика]. Boston, MA: Alekseyev; 2011.

[148] Ambrazevičius R, Wiśniewska I. Chromaticisms or Performance Rules? Evidence from Traditional Singing Pitch transcriptions. J Interdiscip Music Stud 2008;2:19-31.

[149] Yarman O. A Comparative Evaluation of Pitch Notations in Turkish Makam Music: Abjad Scale \& 24-Tone Pythagorean Tuning - 53 Equal Division of the Octave as a Common Grid. J Interdiscip Music Stud 2007;1:43-61. https://doi.org/10/gmnfpw.

[150] Jakobson R. Language in literature. Cambridge MA: Belknap Press; 1987.

[151] Wong PCM, Chan AHD, Roy A, Margulis EH. The Bimusical Brain Is Not Two Monomusical Brains in One: Evidence from Musical Affective Processing. J Cogn Neurosci 2011;23:4082-93. https://doi.org/10/brbcg7.

[152] Verhoef T, Ravignani A. Melodic Universals Emerge or Are Sustained Through Cultural Evolution. Front Psychol 2021;12:668300. https://doi.org/10/gmw3kq.

[153] Ravignani A, Delgado T, Kirby S. Musical evolution in the lab exhibits rhythmic universals. Nat Hum Behav 2016;1:1-7. https://doi.org/10/gfr9gz.

[154] Ravignani A, Thompson B, Grossi T, Delgado T, Kirby S. Evolving building blocks of rhythm: How human cognition creates music via cultural transmission. Ann N Y Acad Sci 2018;1423:176-87. 
https://doi.org/10/ggbm5p.

[155] Alekseyev EYe. Problems in the genesis of musical mode (on the example of Yakut folksong): analysis [Проблемы фрормирования лада (на материале якутской народной песни): исследование]. Moscow: Muzyka; 1976.

[156] Ma W, Fiveash A, Thompson WF. Spontaneous emergence of language-like and music-like vocalizations from an artificial protolanguage. Semiotica 2019;2019:1-23. https://doi.org/10.1515/sem-2018-0139.

[157] Lumaca M, Haumann NT, Vuust P, Brattico E, Baggio G. From random to regular: neural constraints on the emergence of isochronous rhythm during cultural transmission. Soc Cogn Affect Neurosci 2018;13:877-88. https://doi.org/10/gd73qc.

[158] Langlois JH, Roggman L. Attractive faces are only average. Psychol Sci 1990:115-21. https://doi.org/10/fn99mh.

[159] Trujillo LT, Jankowitsch JM, Langlois JH. Beauty is in the ease of the beholding: a neurophysiological test of the averageness theory of facial attractiveness. Cogn Affect Behav Neurosci 2014;14:1061-76. https://doi.org/10/gg2hvs.

[160] Bruckert L, Bestelmeyer P, Latinus M, Rouger J, Charest I, Rousselet GA, et al. Vocal Attractiveness Increases by Averaging. Curr Biol 2010;20:116-20. https://doi.org/10/bpjrtx.

[161] Reigado J, Rocha A, Rodrigues H. Vocalizations of infants (9--11 months old) in response to musical and linguistic stimuli. Int J Music Educ 2011;29:241-55. https://doi.org/10/ftpdjh.

[162] Hargreaves DJ. The Developmental Psychology of Music. Cambridge, UK: Cambridge University Press; 1986.

[163] Newton M. Savage Boys and Wild Girls: A History of Feral Children. London: Faber and Faber; 2002.

[164] Kapoor DS. Socialization and Feral Children. Rev Int Sociol 1973;31:195.

[165] Stendler CB. Critical Periods in Socialization and Overdependency. Child Dev 1952;23:3-12. https://doi.org/10/cgg65w.

[166] Curtiss S, Fromkin V, Krashen S, Rigler D, Rigler M. The Linguistic Development of Genie. Language 1974;50:528-54. https://doi.org/10/bx7rwx.

[167] Fromkin V, Krashen S, Curtiss S, Rigler D, Rigler M. The development of language in Genie: A case of language acquisition beyond the "critical period." Brain Lang 1974;1:81-107. https://doi.org/10/dj8n8p.

[168] Rymer R. Genie: A Scientific Tragedy. Harmondsworth: Penguin; 1994.

[169] Spangler G, Johann M, Ronai Z, Zimmermann P. Genetic and environmental influence on attachment disorganization. J Child Psychol Psychiatry 2009;50:952-61. https://doi.org/10/dt6x49.

[170] Corval R, Mesquita A, Soares I. Links Between Reactive Attachment Disorder, Caregiving and Temperament: A Differential Susceptibility Perspective. J Genet Psychol 2020;181:405-12. https://doi.org/10.1080/00221325.2020.1756206.

[171] Niego A, Benítez-Burraco A. Revisiting the case for 'feral' humans under the light of the human selfdomestication hypothesis 2021. https://doi.org/10.31234/osf.io/wh9qe.

[172] Huron D. Tone and Voice: A Derivation of the Rules of Voice-Leading from Perceptual Principles. Music Percept 2001;19:1-64. https://doi.org/10/c7xtfn.

[173] McAuley JD. Tempo and Rhythm. In: Riess JM, Fay R, Popper A, editors. Springer Handb. Audit. Res., New York: Springer; 2010, p. 165-99. https://doi.org/10.1007/978-1-4419-6114-3_6.

[174] Harrison D. Rhetoric and Fugue: An Analytical Application. Music Theory Spectr 1990;12:1-42. https://doi.org/10/gm22pc.

[175] Pacholczyk J. Early Arab Suite in Spain: An Investigation of the Past Through the Contemporary Living Traditions. Rev Musicol 1993;16:358-66. https://doi.org/10/gmnf3g.

[176] Moreno A. Bossa Nova::Novo Brasil The Significance of Bossa Nova as a Brazilian Popular Music. Lat Am Res Rev 1982;17:129-41.

[177] Maddieson I, Coupé C. Human spoken language diversity and the acoustic adaptation hypothesis. J Acoust Soc Am 2015;138:1838-1838. https://doi.org/10/gnsgpd.

[178] Everett C, Blasi DE, Roberts SG. Climate, vocal folds, and tonal languages: Connecting the physiological and geographic dots. Proc Natl Acad Sci 2015;112:1322-7. https://doi.org/10/gdm7vb.

[179] Roberts SG. Robust, Causal, and Incremental Approaches to Investigating Linguistic Adaptation. Front Psychol 2018;9:166. https://doi.org/10/gc4mjp.

[180] Seliutina IYa, Urtegeshev AYu, Ryzhkova TP, Dambyra ID, Kechil-ool SV. Pharyngealization as a typological feature of phonological systems [Фарингализация как типологический признак фонологических систем]. Novosibirsk, Russia: Omega-Print; 2014.

[181] Kyrgys ZK. Tuvan throat singing [Тувинское горловое пение]. Novosibirsk, Russia: Nauka; 2002.

[182] Wallin NL, Merker B, Brown S. The Origins of Music. Cambridge, MA: MIT Press; 1999.

[183] Schulkin J. Reflections on the musical mind: an evolutionary perspective. Princeton, NJ: Princeton University Press; 2013.

[184] Tomlinson G. A million years of music: the emergence of human modernity. New York, NY: Zone Books; 2015.

[185] Perlovsky L, Nikolsky A, editors. The Evolution of Music. Lausanne: Frontiers Media SA; 2020. https://doi.org/10.3389/978-2-88966-286-9.

[186] Perlovsky L. Music, Passion, and Cognitive Function. Cambridge, MA: Academic Press; 2017. 
[187] Honing $\mathrm{H}$, editor. The Origins of Musicality. Cambridge, MA: The MIT Press; 2019.

[188] Asma S. Music and the Evolution of Embodied Cognition. In: Carroll J, Clasen M, Jonsson E, editors. Evol. Perspect. Imaginative Cult., Berlin: Springer International Publishing; 2020, p. 163-81. https://doi.org/10.1007/978-3-030-46190-4_9.

[189] Fink B, Bläsing B, Ravignani A, Shackelford TK. Evolution and functions of human dance. Evol Hum Behav 2021;42:351-60. https://doi.org/10/gmnfzb.

[190] Andreoni F. Music and Anthropogenic Climate Change: An Evolutionary Perspective 1. vol. 2. 2020.

[191] Patel AD. Music as a Transformative Technology of the Mind: An Update. In: Honing H, editor. Orig. Music., Cambridge, MA: The MIT Press; 2019, p. 113-26. https://doi.org/10.7551/mitpress/10636.003.0009.

[192] Harvey AR. Music, evolution, and the harmony of souls. Oxford, UK: Oxford University Press; 2017. https://doi.org/10.1093/acprof:oso/9780198786856.001.0001.

[193] Jackendoff R. Parallels and Nonparallels between Language and Music. Music Percept 2009;26:195-204. https://doi.org/10/fk7m2x.

[194] Ravignani A, de Boer B. Joint origins of speech and music: testing evolutionary hypotheses on modern humans. Semiotica 2021:169-76. https://doi.org/10/gmnfx9.

[195] Haspelmath M. Human Linguisticality and the Building Blocks of Languages. Front Psychol 2020;0:3056. https://doi.org/10/ggjtqd.

[196] Cross I, Fitch WT, Aboitiz F, Iriki A, Jarvis ED, Lewis J, et al. Culture and Evolution. In: Arbib MA, editor. Lang. Music Brain Mysterious Relatsh., Cambridge, MA: MIT Press; 2013, p. 541-62.

[197] Fitch WT. The Evolution of Language. Cambridge, UK: Cambridge University Press; 2010.

[198] Bowling DL. A vocal basis for the affective character of musical mode in melody. Front Psychol 2013;4:464. https://doi.org/10/gbfpwb.

[199] Dunbar RIM. Bridging the bonding gap: the transition from primates to humans. Philos Trans R Soc B Biol Sci 2012;367:1837-46. https://doi.org/10/f34tg7.

[200] Dunbar RIM. On the evolutionary function of song and dance. In: Bannan N, editor. Music Lang. Hum. Evol., Oxford UK: Oxford University Press; 2012, p. 201-14.

https://doi.org/10.1093/acprof:osobl/9780199227341.003.0008.

[201] Harvey AR. Music and the meeting of human minds. Front Psychol 2018;9. https://doi.org/10/gdkwx6.

[202] Merker B. Synchronous chorusing and human origins. Music Sci 2000;3:59-73. https://doi.org/10/gg5vp6.

[203] Miller DG. Registers in Singing: Empirical and Systematic Studies in the Theory of the Singing Voice. University of Groningen, 2000.

[204] Farrokh K. The Armies of Ancient Persia: The Sassanians. Illustrated edition. Barnsley, South Yorkshire: Pen and Sword Military; 2017.

[205] Jordania J. Why Do People Sing?: Music in Human Evolution. Tbilisi, Georgia: Logos; 2011.

[206] Jordania J. Who Asked the First Question? The Origins of Human Choral Singing, Intelligence, Language and Speech. Tbilisi, Georgia: Logos; 2006.

[207] Nikolsky A. General typology of music texture in the evolutionary earliest forms of music. Commentary on "The 'Musilanguage' Model of Language Evolution." Front Psychol 2018;9:75. https://doi.org/10/gmnfrt.

[208] Agamennone M. Polifonie. Procedimenti, tassonomie e forme: una riflessione "a più voci." Venice: Edizioni II Cardo; 1996.

[209] Nikolsky A, Perlovsky L. Editorial on the Research Topic "The Evolution of Music." The latest important developments. Front Psychol 2020;11:2733. https://doi.org/10/gkztdq.

[210] Saygun AA. Bela Bartok's Folk Music Research in Turkey. Budapest: Akademiai Kiado; 1976.

[211] Saygun AA. Halkevlerinde musiki [Community music]. vol. 6. Ankara, Turkey: Ulusoğlu Basımevi; 1940.

[212] Rouget G. Musical Efficacy: Musicking to Survive-The Case of the Pygmies. Yearb Tradit Music 2011;43:89121. https://doi.org/10/gmnfjt.

[213] Alekseyev EYe. Is there polyphony among the Yakuts? [Есть ли у якутов многоголосие]. Sov Muzyka 1967;5:97-105.

[214] Nettl B. An ethnomusicologist contemplates universals in musical sound and musical culture. In: Wallin NL, Merker B, Brown S, editors. Orig. Music, Cambridge MA: MIT Press; 2000, p. 463-72.

[215] Nettl B. Nettl's Elephant: On the History of Ethnomusicology. Champaign, IL: University of Illinois Press; 2010.

[216] Corballis MC. Crossing the Rubicon: Behaviorism, Language, and Evolutionary Continuity. Front Psychol 2020;11:653. https://doi.org/10/gmnfzr.

[217] Pereira AS, Kavanagh E, Hobaiter C, Slocombe KE, Lameira AR. Chimpanzee lip-smacks confirm primate continuity for speech-rhythm evolution. Biol Lett 2020;16:20200232. https://doi.org/10/ggxrjd.

[218] Cowley SJ, Kuhle A. The rise of languaging. Biosystems 2020;198:104264. https://doi.org/10/gmnfzs.

[219] Hare B, Wobber V, Wrangham R. The self-domestication hypothesis: evolution of bonobo psychology is due to selection against aggression. Anim Behav 2012;83:573-85. https://doi.org/10/fx4xdq.

[220] van der Schyff D, Schiavio A. Evolutionary musicology meets embodied cognition: Biocultural coevolution and the enactive origins of human musicality. Front Neurosci 2017;11:519. https://doi.org/10/gb4r6v.

[221] Heyes C. Cognitive Gadgets. Cambridge, MA: Harvard University Press; 2018. https://doi.org/10.4159/9780674985155.

[222] Dediu D, Levinson SC. On the antiquity of language: The reinterpretation of neandertal linguistic capacities 
and its consequences. Front Psychol 2013;4. https://doi.org/10/gh3x88.

[223] Dediu D, Cysouw M, Levinson SC, Baronchelli A, Christiansen MH, Croft W, et al. Cultural Evolution of Language. In: Richerson PJ, Christiansen MH, editors. Cult. Evol., The MIT Press; 2015, p. 303-32. https://doi.org/10.7551/mitpress/9780262019750.003.0016.

[224] Sinha C. Language and other artifacts: socio-cultural dynamics of niche construction. Front Psychol 2015;6:1601. https://doi.org/10.3389/fpsyg.2015.01601.

[225] Benítez-Burraco A, Progovac L. A four-stage model for language evolution under the effects of human selfdomestication. Lang Commun 2020;73:1-17. https://doi.org/10/gmnfx7.

[226] Benítez-Burraco A. Evolutionary linguistics can help refine (and test) hypotheses about how music might have evolved. Behav Brain Sci 2021:1-8. https://doi.org/10.31234/OSF.IO/KNHDQ.

[227] Brown S. A joint prosodic origin of language and music. Front Psychol 2017;8:1894. https://doi.org/10/gchkg7.

[228] Novik YS. Rite and folklore in Siberian shamanism: an experiment in correlation of structures [Обряд и фольклор в сибирском шаманизме: Опыт сопоставления структур]. Moscow: Eastern Literature, Russian Academy of Science; 2004.

[229] Zemtsovsky I. On creative nature of folklore [О творческой природе фольклора]. In: Kriukov AN, editor. Stylist. Trends Sov. Music 1960-70s Стилевые Тенденции В Советской Музыке 1960-70 Годов, Leningrad: Lenuprizdat; 1979, p. 137-47.

[230] Dahlhaus C. Nineteenth-Century Music. Berkeley, CA: University of California Press; 1989.

[231] Nikolsky A. ¿Cómo funciona la emoción musical? [How Emotion Can Be the Meaning of a Music Work]. In: Cascudo Teresa, editor. Música Cuerpo Estud. Music., Baleares, Spain: Calanda Ediciones Musicales; 2015, p. 241-62. https://doi.org/10.13140/RG.2.1.2737.0008.

[232] Levitin DJ. The World in Six Songs: How the Musical Brain Created Human Nature. New York, USA: Plume Books; 2009.

[233] Trehub SE. Music as a dishonest signal. Behav Brain Sci 2008;31:598-9. https://doi.org/10/ddjhz4.

[234] Juslin PN, Västfjäll D. Emotional responses to music: The need to consider underlying mechanisms. Behav Brain Sci 2008;31. https://doi.org/10/c4nv8d.

[235] Jochelson W. The Yukaghir and the Yukaghirized Tungus. vol. 13. Leiden, The Netherlands: E.J. Brill Itd.; G.E. Stechert; 1910.

[236] Marler P. Three models of song learning: Evidence from behavior. J Neurobiol 1997;33:501-16. https://doi.org/10/fb9zws.

[237] Catchpole CK, Slater PJB. Bird Song: Biological Themes and Variations. Cambridge, MA: Cambridge University Press; 2008.

[238] Mitani JC, Marler P. A phonological analysis of male gibbon singing behavior. Behaviour 1989;109:20-45. https://doi.org/10/bnd52c.

[239] Niemi J, Lapsui A, Lapsui MM. Network of songs: individual songs of the Ob'Gulf Nenets: music and local history as sung by Maria Maksimovna Lapsui. Helsinki: Societe Finno-Ougrienne; 2004.

[240] Alcock J. Animal Behavior: An Evolutionary Approach. Sunderland, MA: Sinauer Associates; 2009.

[241] Temeles EJ. The role of neighbours in territorial systems: when are they "dear enemies"? Anim Behav 1994;47:339-50. https://doi.org/10/bk9cx3.

[242] Schmidt I, Zimmermann A. Population dynamics and socio-spatial organization of the Aurignacian: Scalable quantitative demographic data for western and central Europe. PLOS ONE 2019;14:e0211562. https://doi.org/10/c2s7.

[243] Carto SL, Weaver AJ, Hetherington R, Lam Y, Wiebe EC. Out of Africa and into an ice age: on the role of global climate change in the late Pleistocene migration of early modern humans out of Africa. J Hum Evol 2009;56:139-51. https://doi.org/10/bdnfn6.

[244] Maier A. Population and Settlement Dynamics from the Gravettian to the Magdalenian. Mitteilungen Ges Fuer Urgesch 2017;26:83-102.

[245] Naumov IV. The History of Siberia. London: Routledge; 2006.

[246] Sagalayev AM, Oktiabr'skaya IV. Traditional worldview of Turks of Southern Siberia. Sign and ritual. [Традиционное мировоззрение тюрков Южной Сибири. Знак и ритуал]. Novosibirsk, Russia: Nauka; 1990.

[247] Frolov BA. Primitive Graphics of Europe [Первобытная графика Европы]. Moscow: Nauka; 1992.

[248] Tabarev AV. Human exploration of the Pacific coasts at the turn of the Pleistocene and Holocene [Освоение человеком тихоокеанских побережий на рубеже плейстоцена и голоцена]. Novosibirsk, Russia: The Institute of archaeology and ethnography of Russian Academy of Science; 2004.

[249] Okladnikov AP. Ancient Population of Siberia and Its Cultures. vol. 1. New York, NY: The Peabody Museum; 1959.

[250] Okladnikov AP, editor. The correlation between the ancient cultures of Siberia and the adjacent territories. The collection of works [Соотношение древних культур Сибири и сопредельных территорий. Сборник трудов]. Novosibirsk, Russia: Nauka; 1975.

[251] Okladnikov AP, Derevianko AP. The distant past of Primorye and Priamurye [Далекое прошлое Приморья и Приамурья]. Vladivostok, Russia: The Far-Eastern Book Publishers; 1973.

[252] Okladnikov AP, Shunkov VI. The history of Siberia from the ancient times to the present in 5 vol.: Ancient Siberia [История Сибири с древнейших времен до наших дней в пяти томах: Древняя Сибирь]. vol. 1. 
Leningrad: Nauka; 1968.

[253] Khlobystin LP. Ancient History of the Taymyr Arctic Circle Regions and the Issue of Formation of North Eurasian Cultures [Древняя история Таймырского Заполярья и вопросы формирования культур севера Евразии]. Saint Petersburg: Dmitrii Bulanin Publishers; 1998.

[254] Khlobystin LP. Taymyr: the archaeology of northernmost Eurasia. Washington DC: Arctic Studies Center, National Museum of Natural History, Smithsonian Institution; 2005.

[255] VV P, AN T, EY P, PA N, KE K, RN P. Paleoanthropology. Early human presence in the Arctic: Evidence from 45,000-year-old mammoth remains. Science 2016;351:260-3. https://doi.org/10/bbvr.

[256] Fu Q, Li H, Moorjani P, Jay F, Slepchenko SM, Bondarev AA, et al. Genome sequence of a 45,000-year-old modern human from western Siberia. Nature 2014;514:445-9. https://doi.org/10/f6mv4x.

[257] Wong EHM, Khrunin A, Nichols L, Pushkarev D, Khokhrin D, Verbenko D, et al. Reconstructing genetic history of Siberian and Northeastern European populations. Genome Res 2017;27:1-14. https://doi.org/10/gm39fz.

[258] Sikora M, Pitulko VV, Sousa VC, Allentoft ME, Vinner L, Rasmussen S, et al. The population history of northeastern Siberia since the Pleistocene. Nature 2019;570:182-8. https://doi.org/10/c6wf.

[259] Dobzhanskaya OE. The Living Has Sound; The Dead Is Silent. Anthropol Archeol Eurasia 2016;55:7-21. https://doi.org/10/gf67hg.

[260] Dobzhanskaya O. Ritual musical folklore of Samoyedic peoples as an object of study: main results and research perspectives [Обрядовый музыкальный фольклор самодийских народов как объект изучения: основные результаты и перспективы исследования]. In: Kargin AS, editor. Congr. Congr. Proc. Second Russ. Congr. Folk. Study От Конгресса К Конгрессу Материалы Второго Всероссийского Конгресса Фольклористов, vol. 3, Moscow: State Republican Center of Russian Folklore; 2011, p. 300-11.

[261] Tarasov P, Kvavadze EV, Webb T, Dorofeyuk NI, Bykova GV, Volkova VS, et al. Last glacial maximum biomes reconstructed from pollen and plant macrofossil data from northern Eurasia. J Biogeogr 2000;27:609_ 20. https://doi.org/10/bqk77h.

[262] Hoffecker JF, Elias SA. Human Ecology of Beringia. New York: Columbia University Press; 2007.

[263] Austin MH. The American Rhythm: studies and reexpressions of amerindian songs. Boston, MA: Houghton Mifflin; 1930.

[264] Alekseyev EYe, Nikolayeva N. Models of Yakut vocal folklore [Образцы якутского песенного фольклора]. Yakutsk: Academy of Sciences of the USSR; 1981.

[265] Levin TC. Tuva, Among the Spirits: Sound, Music, and Nature in Sakha and Tuva. New York: Smithsonian Institution; 1999.

[266] List G. Stability and Variation. Ethnomusicology 1987;31:18-34. https://doi.org/10/b8v4pq.

[267] Tischler H. The Evolution of the Harmonic Style in the Notre-Dame Motet. Acta Musicol 1956;28:87. https://doi.org/10/d3kms5.

[268] Fales C. The Paradox of Timbre. Ethnomusicology 2002;46:56-95. https://doi.org/10/bwbncq.

[269] Novik YS. Mythological representations of voice in folklore and beliefs of Siberian реорlеs [Мифологические представления о голосе в фольклоре и верованиях народов Сибири]. In: Ivanov VV, editor. Eurasian Space Sound Word Image Евразийское Пространство Звук Слово Образ, Moscow: Languages of Slavic Cultures; 2003, p. 134-56.

[270] Wiora W. On the Method of Comparative Melodic Research. J Int Folk Music Counc 1957;9:55-8. https://doi.org/10/d858c6.

[271] Zemtsovsky I. Tracing Vesnyanka from Tchaikovsky's Piano Concerto: Historic morphology of a folk song [По следам веснянки из фортепианного концерта П. Чайковского]. Moscow: Muzyka; 1987.

[272] Sipos J. A musical map of different Turkic-speaking peoples, based on field work from 1936 until the present. Bull Int Kodály Soc 2012;37:9-18.

[273] Savage PE. Measuring the cultural evolution of music: With case studies of British-American and Japanese folk, art, and popular music. Tokyo University of the Arts, 2016.

[274] Jan S. "The Two Brothers": Reconciling Perceptual-Cognitive and Statistical Models of Musical Evolution. Front Psychol 2018;9:344. https://doi.org/10/gf7sxs.

[275] Niemi J. Evaluating parameters of structural analysis in indigenous Siberian singing. In: Niemi J, editor. Perspect. Song Indig. Peoples North. Eurasia Perform. Genres Music. Syntax Sound, Tampere, Finland: Tampere University Press; 2009, p. 88-121.

[276] Wilkins AS, Wrangham RW, Fitch WT. The "Domestication Syndrome" in Mammals: A Unified Explanation Based on Neural Crest Cell Behavior and Genetics. Genetics 2014;197:795-808. https://doi.org/10/f6bjcb.

[277] Sánchez-Villagra MR, Schaik CP van. Evaluating the self-domestication hypothesis of human evolution. Evol Anthropol Issues News Rev 2019;28:133-43. https://doi.org/10/gh3x99.

[278] Lord KA, Larson G, Coppinger RP, Karlsson EK. The History of Farm Foxes Undermines the Animal Domestication Syndrome. Trends Ecol Evol 2020;35:125-36. https://doi.org/10/ggfwhj.

[279] Zollikofer CPE, Ponce de León MS. The evolution of hominin ontogenies. Semin Cell Dev Biol 2010;21:44152. https://doi.org/10/bxxjhp.

[280] Shea BT. Heterochrony in human evolution: The case for neoteny reconsidered. Am J Phys Anthropol 1989;32:69-101. https://doi.org/10/bj5rr6.

[281] Leach HM. Human Domestication Reconsidered. Curr Anthropol 2003;44:349-68. https://doi.org/10/b6rsxr. 
[282] Somel M, Franz H, Yan Z, Lorenc A, Guo S, Giger T, et al. Transcriptional neoteny in the human brain. Proc Natl Acad Sci 2009;106:5743-8. https://doi.org/10/dgw9kf.

[283] Plavcan JM. Sexual Size Dimorphism, Canine Dimorphism, and Male-Male Competition in Primates: Where Do Humans Fit In? Hum Nat 2012;23:45-67. https://doi.org/10/ggwbpr.

[284] Fukase $\mathrm{H}$, Kondo O, Ishida $\mathrm{H}$. Size and placement of developing anterior teeth in immature Neanderthal mandibles from Dederiyeh Cave, Syria: Implications for emergence of the modern human chin. Am J Phys Anthropol 2015;156:482-8. https://doi.org/10/f67qhh.

[285] Stringer C. The origin and evolution of Homo sapiens. Philos Trans R Soc B Biol Sci 2016;371:20150237. https://doi.org/10/gfsqs5.

[286] Sánchez-Villagra MR, Geiger M, Schneider RA. The taming of the neural crest: a developmental perspective on the origins of morphological covariation in domesticated mammals. R Soc Open Sci 2016;3:160107. https://doi.org/10/gcx732.

[287] Shilton D, Breski M, Dor D, Jablonka E. Human Social Evolution: Self-Domestication or Self-Control? Front Psychol 2020;11:134. https://doi.org/10/gh23n8.

[288] Gleeson BT, Kushnick G. Female status, food security, and stature sexual dimorphism: Testing mate choice as a mechanism in human self-domestication. Am J Phys Anthropol 2018;167:458-69. https://doi.org/10/gd5db5.

[289] Wrangham RW. Two types of aggression in human evolution. Proc Natl Acad Sci 2018;115:245-53. https://doi.org/10/gctvgc.

[290] Cieri RL, Churchill SE, Franciscus RG, Tan J, Hare B. Craniofacial Feminization, Social Tolerance, and the Origins of Behavioral Modernity. Curr Anthropol 2014;55:419-43. https://doi.org/10/gcx737.

[291] Benítez-Burraco A, Chekalin E, Bruskin S, Tatarinova T, Morozova I. Recent selection of candidate genes for mammal domestication in Europeans and language change in Europe: a hypothesis. Ann Hum Biol 2021. https://doi.org/10/gmnfzk.

[292] Sala N, Arsuaga JL, Pantoja-Pérez A, Pablos A, Martínez I, Quam RM, et al. Lethal Interpersonal Violence in the Middle Pleistocene. PLOS ONE 2015;10:e0126589. https://doi.org/10/4w6.

[293] Frayer D, Estabrook V. Trauma in the Krapina Neandertals: Violence in the Middle Palaeolithic? In: Knüsel C, Smith M, editors. Routledge Handb. Bioarchaeology Hum. Confl., London: Taylor and Francis; 2013, p. 6789.

[294] Trinkaus E. Neandertals, early modern humans, and rodeo riders. J Archaeol Sci 2012;39:3691-3. https://doi.org/10/gf7qkx.

[295] Gamble C. The Palaeolithic societies of Europe. Cambridge, UK: Cambridge University Press; 1999.

[296] Sykes RW. Kindred: Neanderthal Life, Love, Death and Art. London, UK; Oxford ; New York: Bloomsbury Sigma; 2020.

[297] Pisor AC, Surbeck M. The evolution of intergroup tolerance in nonhuman primates and humans. Evol Anthropol Issues News Rev 2019;28:210-23. https://doi.org/10/gmnf2d.

[298] Raia P, Mondanaro A, Melchionna M, Di Febbraro M, Diniz-Filho JAF, Rangel TF, et al. Past Extinctions of Homo Species Coincided with Increased Vulnerability to Climatic Change. One Earth 2020;3:480-90. https://doi.org/10/fdnq.

[299] Spikins P, French JC, John-Wood S, Dytham C. Theoretical and Methodological Approaches to Ecological Changes, Social Behaviour and Human Intergroup Tolerance 300,000 to 30,000 BP. J Archaeol Method Theory 2021;28:53-75. https://doi.org/10/gmnf2g.

[300] Ostapuschenko AA, Petrova YA. Hospitality of the peoples of the North Caucasus as one of the elements of ethnic culture. Sci Alm Black Sea Reg Ctries 2019;20:22-8. https://doi.org/10/gm499x.

[301] Czaplicka M. Aboriginal Siberia, A study in Social Anthropology. Oxford, UK: Clarendon Press; 1914.

[302] Kjellstroem R. Eskimo Marriage: An Account of Traditional Eskimo Courtship and Marriage. vol. 80. Stockholm, Sweden: Nordiska museet; 1973.

[303] Bogoraz-Tan VG (Bogoras. The Chukchee: material culture [part 1], religion [part 2], social organization [part 3] $1904 ;$ XI.

[304] Jamison SW. Sacrificed Wife/Sacrificer's Wife: Women, Ritual, and Hospitality in Ancient India. 1st edition. New York: Oxford University Press; 1996.

[305] O'Gorman K. Dimensions of Hospitality: Exploring Ancient and Classical Origins. In: Lashley C, Lynch P, Morrison AJ, editors. Hosp. Soc. Lens, Amsterdam, Boston, Heidelberg: Elsevier; 2007, p. 17-32.

[306] Hennigh L. Functions and Limitations of Alaskan Eskimo Wife Trading. Arctic 1970;23:24-34. https://doi.org/10.14430/arctic315110/gm5fwh.

[307] Matthews VH. Old Testament Parallels: Laws and Stories from the Ancient Near East. 3rd edition. New York: Paulist Press; 2007.

[308] Reece S. The Stranger's Welcome: Oral Theory and the Aesthetics of the Homeric Hospitality Scene. Ann Arbor: University of Michigan Press; 1993.

[309] Jipp JW. Divine Visitations and Hospitality to Strangers in Luke-Acts. Brill; 2013. https://doi.org/10.1163/9789004258006_004.

[310] Selwyn T. An anthropology of hospitality, 2001, p. 18-37. https://doi.org/10.1016/B978-0-7506-5431-9.500068.

[311] Gluckman M. Custom and Conflict in Africa. New York, NY: Barnes \& Noble; 1956. 
[312] Prüfer K, de Filippo C, Grote S, Mafessoni F, Korlević P, Hajdinjak M, et al. A high-coverage Neandertal genome from Vindija Cave in Croatia. Science 2017;358:655-8. https://doi.org/10.1126/science.aao1887.

[313] Mafessoni F, Grote S, Filippo C de, Slon V, Kolobova KA, Viola B, et al. A high-coverage Neandertal genome from Chagyrskaya Cave. Proc Natl Acad Sci 2020;117:15132-6. https://doi.org/10/gg22k7.

[314] Hublin J-J. Climatic Changes, Paleogeography, and the Evolution of the Neandertals. In: Akazawa T, Aoki K, Bar-Yosef O, editors. Neandertals Mod. Hum. West. Asia, Boston, MA: Springer US; 1998, p. $295-310$. https://doi.org/10.1007/0-306-47153-1_18.

[315] Reich D, Green RE, Kircher M, Krause J, Patterson N, Durand EY, et al. Genetic history of an archaic hominin group from Denisova Cave in Siberia. Nature 2010;468:1053-60. https://doi.org/10/c2z.

[316] Meyer M, Kircher M, Gansauge M-T, Li H, Racimo F, Mallick S, et al. A high-coverage genome sequence from an archaic Denisovan individual. Science 2012;338:222-6. https://doi.org/10/q8p.

[317] Qin P, Stoneking M. Denisovan Ancestry in East Eurasian and Native American Populations. Mol Biol Evol 2015;32:2665-74. https://doi.org/10/f7sz54.

[318] Massilani D, Skov L, Hajdinjak M, Gunchinsuren B, Tseveendorj D, Yi S, et al. Denisovan ancestry and population history of early East Asians. Science 2020;370:579-83. https://doi.org/10/gm52zr.

[319] Zhang D, Xia H, Chen F, Li B, Slon V, Cheng T, et al. Denisovan DNA in Late Pleistocene sediments from Baishiya Karst Cave on the Tibetan Plateau. Science 2020;370:584-7. https://doi.org/10/ghhtqb.

[320] Zhang X, Witt KE, Bañuelos MM, Ko A, Yuan K, Xu S, et al. The history and evolution of the DenisovanEPAS1 haplotype in Tibetans. Proc Natl Acad Sci 2021;118. https://doi.org/10.1073/pnas.2020803118.

[321] Sawyer S, Renaud G, Viola B, Hublin J-J, Gansauge M-T, Shunkov MV, et al. Nuclear and mitochondrial DNA sequences from two Denisovan individuals. Proc Natl Acad Sci U S A 2015;112:15696-700. https://doi.org/10/gfb79m.

[322] Slon V, Mafessoni F, Vernot B, de Filippo C, Grote S, Viola B, et al. The genome of the offspring of a Neanderthal mother and a Denisovan father. Nature 2018;561:113-6. https://doi.org/10/cs64.

[323] Peter BM. 100,000 years of gene flow between Neandertals and Denisovans in the Altai mountains. 2020.

[324] Kuhlwilm M, Gronau I, Hubisz MJ, de Filippo C, Prado-Martinez J, Kircher M, et al. Ancient gene flow from early modern humans into Eastern Neanderthals. Nature 2016;530:429-33. https://doi.org/10/bhjt.

[325] Sankararaman S, Mallick S, Patterson N, Reich DE. The Combined Landscape of Denisovan and Neanderthal Ancestry in Present-Day Humans. Curr Biol 2016;26:1241-7. https://doi.org/10/f8kcz7.

[326] Been E, Hovers E, Ekshtain R, Malinski-Buller A, Agha N, Barash A, et al. The first Neanderthal remains from an open-air Middle Palaeolithic site in the Levant. Sci Rep 2017;7:2958. https://doi.org/10.1038/s41598-01703025-z.

[327] Gowlett J a. J. Kinship (Early Human), the Archaeological Evidence for. Int. Encycl. Anthropol., American Cancer Society; 2018, p. 1-19. https://doi.org/10.1002/9781118924396.wbiea2343.

[328] Yang MA, Fu Q. Insights into Modern Human Prehistory Using Ancient Genomes. Trends Genet 2018;34:184-96. https://doi.org/10/gdpd3z.

[329] Zilhão J. The Upper Palaeolithic of Europe. In: Renfrew C, Bahn PG, editors. Camb. World Prehistory, vol. 3, Cambridge, UK: Cambridge University Press; 2014, p. 1753-85. https://doi.org/10.1017/cho9781139017831.105.

[330] Barnard A. The Co-Evolution of Language and Kinship. Early Hum. Kinsh., Malden, MA: John Wiley \& Sons, Ltd; 2008, p. 232-43. https://doi.org/10.1002/9781444302714.ch13.

[331] Funk DA. Economic activities and materialized culture [Хозяйственные занятия и материализованная культура]. In: Funk DA, Tomilov NA, editors. Turk. Peoples Sib. Тюркские Народы Сибири, Мoscow: Nauka; 2006, p. 186-204.

[332] Wilson RA. Rethinking Incest Avoidance: Beyond the Disciplinary Groove of Culture-First Views. Biol Theory 2021;16:162-75. https://doi.org/10/gm5t4j.

[333] Lambeck K, Rouby H, Purcell A, Sun Y, Sambridge M. Sea level and global ice volumes from the Last Glacial Maximum to the Holocene. Proc Natl Acad Sci 2014;111:15296-303. https://doi.org/10.1073/pnas.1411762111.

[334] Pitulko V, Pavlova E, Nikolskiy P. Revising the archaeological record of the Upper Pleistocene Arctic Siberia: Human dispersal and adaptations in MIS 3 and 2. Quat Sci Rev 2017;165:127-48. https://doi.org/10/gf9xjz.

[335] Holmes CE. Tanana River Valley Archaeology circa 14,000 to 9000 B.P. Arct Anthropol 2001;38:154-70.

[336] Willerslev E, Meltzer DJ. Peopling of the Americas as inferred from ancient genomics. Nature 2021;594:35664. https://doi.org/10/gkn9d5.

[337] Raghavan M, Skoglund P, Graf KE, Metspalu M, Albrechtsen A, Moltke I, et al. Upper Palaeolithic Siberian genome reveals dual ancestry of Native Americans. Nature 2014;505:87-91. https://doi.org/10/f23mmt.

[338] Yu H, Spyrou MA, Karapetian M, Shnaider S, Radzevičiūtè R, Nägele K, et al. Paleolithic to Bronze Age Siberians Reveal Connections with First Americans and across Eurasia. Cell 2020;181:1232-1245.e20. https://doi.org/10.1016/j.cell.2020.04.037.

[339] Bar-Yosef O. Neanderthals and Modern Humans Across Eurasia. Dyn. Learn. Neanderthals Mod. Hum., vol. 1, 2013, p. 7-20.

[340] Schurr TG. Tracing Human Movements from Siberia to the Americas: Insights from Genetic Studies. In: Frachetti MD, Spengler RNI, editors. Mobil. Anc. Soc. Asia Am., Cham: Springer International Publishing; 2015, p. 23-47. https://doi.org/10.1007/978-3-319-15138-0_4. 
[341] Washburne C, Derno M, editors. Bad music: the music we love to hate. New York: Routledge; 2004.

[342] Snowdon CT. Expression of emotion in nonhuman animals. In: Davidson RJ, Scherer KR, Goldsmith HH, editors. Handb. Affect. Sci., Oxford, UK: Oxford University Press; 2003, p. 457-80.

[343] Hirsh-Pasek K, Treiman R. Doggerel: motherese in a new context. J Child Lang 1982;9:229-37. https://doi.org/10/cdc7zc.

[344] Mitchell RW. Americans' Talk to Dogs: Similarities and Differences With Talk to Infants. Res Lang Soc Interact 2001;34:183-210. https://doi.org/10/b2zq2s.

[345] Trevarthen C. The musical art of infant conversation: Narrating in the time of sympathetic experience, without rational interpretation, before words. Music Sci 2008;12:15-46. https://doi.org/10/cxw457.

[346] O'Rourke T, Martins PT, Asano R, Tachibana RO, Okanoya K, Boeckx C. Capturing the Effects of Domestication on Vocal Learning Complexity. Trends Cogn Sci 2021;25:462-74. https://doi.org/10/gmnf2c.

[347] Nettl B, Levine VL, Keillor E. Amerindian music. Grove Music Online 2001;1. https://doi.org/10.1093/GMO/9781561592630.ARTICLE.45405.

[348] Nettl B, Myers H. Folk Music in the United States: An Introduction. Wayne State University Press; 1976.

[349] Nettl B. Cheremis Musical Styles. Indiana University Press; 1960.

[350] Szomjas-Schiffert G. Traditional Singing Style of the Lapps. Yearb Int Folk Music Counc 1973;5:51-61. https://doi.org/10/dkm437.

[351] Olsen PR. Acculturation in the Eskimo Songs of the Greenlanders. Yearb Int Folk Music Counc 1972;4:32-7. https://doi.org/10/ftc6pb.

[352] Johnston TF. Eskimo Music by Region: A Comparative Circumpolar Study. Ottawa: University of Ottawa Press; 1976.

[353] Bhattacharya S. Ethno-musicology and India. Calcutta: Indian Publications; 1968.

[354] Wiora W. Older than Pentatony. In: Rejeczky B, editor. Stud. Memoriae Bela Bartok Sacra, London: Boosey and Hawkes; 1959, p. 183-206.

[355] McLean M, McLean AP of E and H of A of M and PMM. Maori Music. Auckland University Press; 1996.

[356] McLean M. Weavers of Song: Polynesian Music and Dance. Honolulu, HI: University of Hawai'i Press; 1999.

[357] Kunst J. Music in New Guinea: three studies. 'S-Gravenhage: Martinus Nijhoff.; 1967.

[358] McLean M. Music, Lapita, and the Problem of Polynesian Origins. Mervyn McLean; 2014.

[359] Miller GA. The magical number seven, plus or minus two: some limits on our capacity for processing information. Psychol Rev 1994;101:343-52. https://doi.org/10/d66f6q.

[360] Mathy F, Feldman J. What's Magic about Magic Numbers? Chunking and Data Compression in Short-Term Memory. Cognition 2012;122:346-62. https://doi.org/10/ds3s2z.

[361] Bolender J. Prehistoric cognition by description: A Russellian approach to the upper paleolithic. Biol Philos 2007;22:383-99. https://doi.org/10/d4w63f.

[362] Wray A, Grace GW. The consequences of talking to strangers: Evolutionary corollaries of socio-cultural influences on linguistic form. Lingua 2007;117:543-78. https://doi.org/10/d9mz43.

[363] Lupyan G, Dale R. Language Structure Is Partly Determined by Social Structure. PLOS ONE 2010;5:e8559. https://doi.org/10/bxx7rm.

[364] Trudgill Peter. Sociolinguistic typology: social determinants of linguistic complexity. Oxford, UK: Oxford University Press; 2011.

[365] Nettle D. Social scale and structural complexity in human languages. Philos Trans R Soc B Biol Sci 2012;367:1829-36. https://doi.org/10/gfzkv7.

[366] Asano R. The evolution of hierarchical structure building capacity for language and music: a bottom-up perspective. Primates 2021. https://doi.org/10/gmkz22.

[367] Benítez-Burraco A, Progovac L. Reconstructing prehistoric languages. Philos Trans R Soc B Biol Sci 2021;376:20200187. https://doi.org/10/gnbvgd.

[368] Benítez-Burraco A, Progovac L. Language evolution: examining the link between cross-modality and aggression through the lens of disorders. Philos Trans R Soc B Biol Sci 2021;376:20200188. https://doi.org/10/gns57p.

[369] Progovac L, Benítez-Burraco A. From Physical Aggression to Verbal Behavior: Language Evolution and SelfDomestication Feedback Loop. Front Psychol 2019;10:2807. https://doi.org/10/gnbvgg.

[370] Bargeliotes L. Relevant Hellenic Factors Favoring Effective Dialogue and Peaceful Coexistence. Am J Econ Sociol 2009;68:205-30. https://doi.org/10/dmrmf8.

[371] Foley M. Of Violence and Rhetoric: An Ethical Aporia. Q J Speech 2013;99:191-9. https://doi.org/10/gnss7w.

[372] West ML. Ancient Greek music. New York, London: Oxford University Press; 1992.

[373] Robertson N. Orphic Mysteries and Dionysiac ritual. In: Cosmopoulos M, editor. Greek Mysteries Archaeol. Ritual Anc. Greek Secret Cults, London: Routledge; 2002.

[374] Isler-Kerényi C. New Contributions of Dionysiac Iconography to the History of Religions in Greece and Italy. In: Casadio G, Johnston PA, editors. Mystic Cults Magna Graecia, Dallas, TX: University of Texas Press; 2009, p. 61-72. https://doi.org/10.7560/719026-006.

[375] West ML. The Singing of Homer and the Modes of Early Greek Music. J Hell Stud 1981;101:113-29. https://doi.org/10/dmwpnn.

[376] Csapo EG. The politics of the new music. In: Murray P, Wilson P, editors. Music Muses Cult. Mousikē Class. Athen. City, Oxford, UK: Oxford University Press; 2004, p. 207-48. 
[377] Wiora W. The four ages of music. New York: W.W. Norton \& Co.; 1965.

[378] Szabolcsi B. History of Melody. Budapest: Barrie \& Rockliff; 1965.

[379] Sachs C. The wellsprings of music. The Hague, Netherlands: Martinus Nijhoff; 1962.

[380] Kvitka KV. Selected Works [Избранные труды]. vol. 1. Moscow: Soviet Composer; 1971.

[381] Myers H. Ethnomusicology: Historical and Regional Studies. New York, NY: Norton; 1993. https://doi.org/papers3://publication/uuid/02C6B958-898E-4EBB-AA11-89FCCA77E3B7.

[382] Sheikin YI. History of world musical culture: from archaic times to the 16th century AD. [История мировой музыкальной культуры: от древних времен до XVI века н.э.]. Yakutsk, Russia: The Ministry of culture and spiritual development of Sakha Republic; 2017.

[383] Nikolsky A. Evolution of tonal organization in music mirrors symbolic representation of perceptual reality. Part1: Prehistoric. Front Psychol 2015;6. https://doi.org/10/f7wvp8.

[384] Lippman EA. Musical Thought in Ancient Greece. New York: Da Capo Press; 1964.

[385] Noorden L van. Temporal Coherence in the Perception of Tone Sequences. vol. 3. Eindhoven, Holland: Institute for Perceptual Research; 1975.

[386] Nikolsky A. Can folk music be chromatic?: "natural" microtonal modes, chromatic degrees and alterations. Front Psychol 2016;7:18. https://doi.org/10/gmnfzf.

[387] Starostina T. Modal systematization of Russian traditional song [Ладовая систематика русской народной песни]. Harmony Probl. Sci. Methodol. Гармония Проблемы Науки И Методики, vol. 1, Moscow: Muzyka; 1973, p. 85-105.

[388] Sachs C. Primitive and medieval music: a parallel. J Am Musicol Soc 1960;13:43-9.

[389] Rudneva A. Russian traditional musical works: essays on the theory of folklore [Русское народное музыкальное творчество: очерки по теории фольклора]. Moscow: Kompozitor; 1994.

[390] Goshovsky VL. At the origins of Slavic folk music [У истоков народной музыки славян]. Moscow: Soviet Composer; 1971.

[391] Kodály Z. Folk music of Hungary. New York, NY: Da Capo Press; 1987.

[392] Leisiö T. On old-Lithuanian modalities: A hypothesis on five stylistic strata based on proto-Indo-European pentatonic roots. In: Astrauskas R, editor. Ethn. Relat. Music. Folk., Vilnius: Lietuvos Muzikos Akademija; 2002, p. 22-51.

[393] Katsanevaki AN. Archaic elements in the vocal musical tradition of the mountain populations of northern Greece: Results of research conducted among the Vlach-speaking and Greek-speaking populations of the mountain region of Northern Pindus, Greece. Muzikol Časopis Muzikološkog Instituta Srp Akad Nauka Umet 2005;5:207-44.

[394] Sipos J. Azeri Folksongs: At the Fountain-head of Music. Budapest: Akademiai Kiado; 2004.

[395] Nikolsky A. Tonal Organization in Tuning of Paleolithic and Neolithic Pipes. Front Psychol 2015;6:14. https://doi.org/10.6084/m9.figshare.13636313.

[396] Chou W-C. Chinese historiography and music: some observations. Music Q 1976;62:218-40. https://doi.org/10/ddgb9k.

[397] Petrunina MO. On formation of the modal pitch system in folk songs of China [О формировании звуковысотно-ладовой системы народных песен Китая]. Bull Kemerovo State Univ Cult Arts 2009:71-8.

[398] Lomax A. Universals in Song. World Music 1977;19:117-29.

[399] Kubik G. Africa and the Blues. Jackson, MS: University Press of Mississippi; 1999.

[400] Davidson M. Some music for the Lala Kankobele. Afr Music J Afr Music Soc 1970;4:103-13. https://doi.org/10/gmnfnx.

[401] Berliner PF. The Soul of the Mbira: Music and Traditions of the Shona People of Zimbabwe. 1993.

[402] Arom S, Voisin F. Theory and Technology in African Music. In: Stone RM, editor. Garland Encycl. World Music Afr., vol. 1, New York, NY: Garland Publishing; 1997, p. 254-70.

[403] Seeger C. Studies in Musicology, 1935-1975. University of California Press; 1977.

[404] Huron D. Sweet Anticipation: Music and the Psychology of Expectation. Cambridge, MA: MIT Press; 2006.

[405] Krumhansl CL, Cuddy LL. A Theory of Tonal Hierarchies in Music. In: Riess Jones M, Fay RR, Popper AN, editors. Music Percept. Springer Handb. Audit. Res. 36, vol. 36, Springer New York; 2010, p. 51-87. https://doi.org/10.1007/978-1-4419-6114-3.

[406] Krumhansl CL. Cognitive foundations of musical pitch. New York: Oxford University Press; 1990. https://doi.org/10.1121/1.404005.

[407] Lerdahl F. Genesis and Architecture of the GTTM Project. Music Percept 2009;26:187-94. https://doi.org/10/c6cd29.

[408] Larson S. Musical Forces: Motion, Metaphor, and Meaning in Music. Bloomington, IN: Indiana University Press; 2012.

[409] Bharucha JJ. Melodic anchoring. Music Percept 1996;13:383-400. https://doi.org/10/gmnfg3.

[410] Blacking J. Venda Children's Songs: A Study in Ethnomusicological Analysis. Chicago: University of Chicago Press; 1967.

[411] Holzmann R. Q'ero, pueblo y música: un estudio etnomusicológico basado en 33 piezas del repertorio vocal e instrumental de los q'eros. Lima: Prólogo; 1986.

[412] Holzmann R. Cuatro Ejemplos de Música Q'ero (Cuzco, Perú). Lat Am Music Rev Rev Música Latinoam 1980;1:74-91. https://doi.org/10/c94hkf. 
[413] Wissler H. From Grief and Joy We Sing: Social and Cosmic Regenerative Processes in the Songs of Q'Eros, Peru. Florida State University, 2009.

[414] Romero RR. Sonidos Andinos. Una antología de la música campesina del Perú. Pontificia Universidad Católica del Perú. Instituto Riva-Agüero. Centro de Etnomusicología Andina; 2002.

[415] Arnold DY, Yapita JDD. Rio de vellon, rio de canto: Cantar a los animales, una poetica andina de la creacion (Coleccion Academia). La Paz, Bolivia: Facultad de Humanidades y Ciencias de la Educacion; 1998.

[416] Kuss M, editor. Music in Latin America and the Caribbean: An Encyclopedic History Volume 1: Performing Beliefs: Indigenous Peoples of South America, Central America, and Mexico. Denver, TX: University of Texas Press; 2004.

[417] Danneman M. Autochthonous Musical Culture in Chile. World Music 1984;26:68-77.

[418] Fenk-Oczlon G. What Vowels Can Tell Us about the Evolution of Music. Front Psychol 2017;8:1581. https://doi.org/10/gbxtvj.

[419] Ogolevets A. The Basics of Harmonic Language [Основы гармонического языка]. Moscow: Muzgiz; 1941.

[420] Kolinsky R, Lidji P, Peretz I, Besson M, Morais J. Processing interactions between phonology and melody: Vowels sing but consonants speak. Cognition 2009;112:1-20. https://doi.org/10/bmvx62.

[421] Benítez-Burraco A, Kempe V. The emergence of modern languages: Has human self-domestication optimized language transmission? Front Psychol 2018;9:551. https://doi.org/10/gmjzpj.

[422] Dumbrill R. The Musicology and Organology of the Ancient Near East. London: Tadema Press; 1998.

[423] Foster BR. The Person in Mesopotamian Thought. In: Radner K, Robson E, editors. Oxf. Handb. Cuneif. Cult., Oxford, UK: Oxford University Press; 2011, p. 117-39.

[424] Michalowski P. Literacy, Schooling and the Transmission of Knowledge in Early Mesopotamian Culture. In: Egmond WSV, Soldt WHV, editors. Theory Pract. Knowl. Transf. Stud. Sch. Educ. Anc. East Beyond, Leiden: Nederlands Institut Voor Het Nabije Oosten; 2012, p. 39-5819.

[425] Hallo WW. The world's oldest literature: studies in Sumerian belles-lettres. Leiden, The Netherlands: Koniklijke Brill NV; 2010.

[426] Veldhuis N. Levels of literacy. In: Radner K, Robson E, editors. Oxf. Handb. Cuneif. Cult., Oxford, UK: Oxford University Press; 2011, p. 68-89.

[427] Franklin JC. The Global Economy of Music in the Ancient Near East. In: Westenholz JG, editor. Sounds Anc. Music, Jerusalem: Bible Lands Museum; 2007, p. 27-37.

[428] Wrangham RW, Glowacki L. Intergroup Aggression in Chimpanzees and War in Nomadic Hunter-Gatherers. Hum Nat 2012;23:5-29. https://doi.org/10/ggkwt2.

[429] Choi JK, Bowles S. The coevolution of parochial altruism and war. Science 2007;318:636-40. https://doi.org/10/cwtwng.

[430] Ferring R, Oms O, Agustí J, Berna F, Nioradze M, Shelia T, et al. Earliest human occupations at Dmanisi (Georgian Caucasus) dated to 1.85-1.78 Ma. Proc Natl Acad Sci 2011;108:10432-6. https://doi.org/10/cgp7d3.

[431] Leroy SAG, Arpe K, Mikolajewicz U. Vegetation context and climatic limits of the Early Pleistocene hominin dispersal in Europe. Quat Sci Rev 2011;30:1448-63. https://doi.org/10/dkr9jc.

[432] Boaz NT, Ciochon RL. Dragon Bone Hill: An Ice-Age Saga of Homo erectus. Oxford, UK: Oxford University Press; 2004.

[433] Clark PU, Archer D, Pollard D, Blum JD, Rial JA, Brovkin V, et al. The middle Pleistocene transition: characteristics, mechanisms, and implications for long-term changes in atmospheric pCO2. Quat Sci Rev 2006;25:3150-84. https://doi.org/10/bk9cm4.

[434] Antón SC, Leigh SR. Growth and life history in Homo erectus. In: Nelson AJ, Krovitz GE, Thompson JL, editors. Patterns Growth Dev. Genus Homo, Cambridge: Cambridge University Press; 2003, p. 219-45. https://doi.org/10.1017/CBO9780511542565.009.

[435] Jackendoff R. Possible stages in the evolution of the language capacity. Trends Cogn Sci 1999;3:272-9. https://doi.org/10/cfj4bj.

[436] Falk D. Prelinguistic evolution in early hominins: Whence motherese? Behav Brain Sci 2004;27:491-503. https://doi.org/10/dvbfj5.

[437] Pagel M. Q\&amp;A: What is human language, when did it evolve and why should we care? BMC Biol 2017;15:64. https://doi.org/10/ffs8.

[438] Mithen SJ. The Singing Neanderthals: The Origins of Music, Language, Mind, and Body. Cambridge, MA: Harvard University Press; 2005.

[439] Kraus N, Slater J. Music and language. In: Aminoff MJ, Boller F, Swaab DF, editors. Handb. Clin. Neurol., vol. 129, Amsterdam, Netherlands: Elsevier; 2015, p. 207-22. https://doi.org/10.1016/B978-0-444-626301.00012-3.

[440] Rohrmeier M, Zuidema W, Wiggins GA, Scharff C. Principles of structure building in music, language and animal song. Philos Trans R Soc B Biol Sci 2015;370:20140097. https://doi.org/10/f637t2.

[441] Bancel P, L'Etang AM, Bengtson J. Back to Proto-Sapiens (part 2): The global kinship terms papa, mama, and kaka. In: Milicic B, Jones D, editors. Kinsh. Lang. Prehistory Hage Renaiss. Kinsh. Stud., Salt Lake City: University of Utah Press; 2015, p. 38-45.

[442] de l'Etang M, Bancel P, Ruhlen M. Back to Proto-Sapiens (Part 1). In: Jones D, Milicic B, editors. Kinsh. Lang. Prehistory Hage Renaiss. Kinsh. Stud., Salt Lake City: University of Utah Press; 2015, p. 29-37. 
[443] Rácz P, Passmore S, Sheard C, Jordan FM. Usage frequency and lexical class determine the evolution of kinship terms in Indo-European. R Soc Open Sci 2019;6:191385. https://doi.org/10.1098/rsos.191385.

[444] Jordania J. Music and emotion: Humming in the beginning of human history. In: Tsurtsumia R, editor. Fourth Int. Symp. Tradit. Polyphony Tbilisi Ga., New York: Nova Science; 2008, p. 41-9. https://doi.org/2008-26427.

[445] Montagu J. How old is music? Galpin Soc J 2004;57:171-82.

[446] Duncan-Kemp AM. Where Strange Paths Go Down. Brisbane, Australia: W. R. Smith \& Paterson; 1952.

[447] Boivin N, Brumm A, Lewis H, Robinson D, Korisettar R. Sensual, material, and technological understanding: exploring prehistoric soundscapes in south India. J R Anthropol Inst 2007;13:267-94. https://doi.org/10/dpghp2.

[448] Wurz S. Interpreting the fossil evidence for the evolutionary origins of music. South Afr Humanit 2010;21:395417.

[449] Dubreuil B. Paleolithic public goods games: Why human culture and cooperation did not evolve in one step. Biol Philos 2010;25:53-73. https://doi.org/10/cjsf5q.

[450] Goren-Inbar N, Alperson N, Kislev ME, Simchoni O, Melamed Y, Ben-Nun A, et al. Evidence of Hominin Control of Fire at Gesher Benot Ya'aqov, Israel. Science 2004;304:725-7. https://doi.org/10/csvv2s.

[451] Maclarnon A, Hewitt G. Increased breathing control: Another factor in the evolution of human language. Evol Anthropol Issues News Rev 2004;13:181-97. https://doi.org/10/bgpnct.

[452] Martínez I, Rosa M, Quam R, Jarabo P, Lorenzo C, Bonmatí A, et al. Communicative capacities in Middle Pleistocene humans from the Sierra de Atapuerca in Spain. Quat Int 2013;295:94-101. https://doi.org/10/f4z6p4.

[453] Martínez I, Rosa M, Arsuaga JL, Jarabo P, Quam R, Lorenzo C, et al. Auditory capacities in Middle Pleistocene humans from the Sierra de Atapuerca in Spain. Proc Natl Acad Sci U S A 2004;101:9976-81. https://doi.org/10/drxtvc.

[454] Bowling DL. The Biological Basis of Emotion in Musical Tonality. Duke University, 2012. http://hdl.handle.net/10161/5522.

[455] Conde-Valverde M, Martínez I, Quam RM, Rosa M, Velez AD, Lorenzo C, et al. Neanderthals and Homo sapiens had similar auditory and speech capacities. Nat Ecol Evol 2021;5:609-15. https://doi.org/10/fxrg.

[456] Ahlner F, Zlatev J. Cross-modal iconicity: A cognitive semiotic approach to sound symbolism. Sign Syst Stud 2010;38:298-348. https://doi.org/10/ggvc8n.

[457] Marks LE. The unity of the senses: interrelations among the modalities. New York: Academic Press; 1978.

[458] Nuckolls JB. To be or not to be ideophonically impoverished. In: Chiang WF, Chun E, Mahalingappa L, Mehus S, editors. Proc. Elev. Annu. Symp. Lang. Soc. - Austin Tex. Linguist. Forum, Austin, Texas: Texas Linguistic Forum; 2004, p. 131-42.

[459] Monaghan P, Shillcock RC, Christiansen MH, Kirby S. How arbitrary is language? Philos Trans R Soc Lond B Biol Sci 2014;369:20130299. https://doi.org/10/f6p6g7.

[460] Powers HS, Wiering F. Mode. The term. Medieval modal theory. Modal theories and polyphonic music. New Grove Dict Music Music 2001. https://doi.org/10.1093/gmo/9781561592630.article.43718.

[461] Ehlers J, Gibbard PL. Quaternary Glaciations - Extent and Chronology. Part I: Europe. vol. 1. Amsterdam: Elsevier; 2004.

[462] Ehlers J, Gibbard PL. Quaternary Glaciations - Extent and Chronology. Part III: South America, Asia, Africa, Australasia, Antarctica. vol. 3. Amsterdam: Elsevier; 2004.

[463] Cox CB, Healey IN, Moore PD. Biogeography: an ecological and evolutionary approach. Hoboken, NJ: Wiley; 2016.

[464] Yesipova MV. Phono-instruments (Ethnophones). Music Instrum Музыкальные Инструменты 2008:633.

[465] Sheikin YI. Musical culture of peoples of Northern Asia [Музыкальная культура народов Северной Азии]. Yakutsk: Yakutskii scientific center; 1996.

[466] Spelke ES. What makes us smart? Core knowledge and natural language. Lang. Mind Adv. Study Lang. Thought, Cambridge, MA, US: MIT Press; 2003, p. 277-311.

[467] Spelke ES, Kinzler KD. Core knowledge. Dev Sci 2007;10:89-96. https://doi.org/10/fhpxzt.

[468] Rutkowski J. The Nature of Children's Singing Voices: Characteristics and Assessment. In: Roberts BA, editor. Phenom. Sing., St. John's, NF: Memorial University Press; 1997, p. 201-9.

[469] McKernon PE. The development of first songs in young children. New Dir Child Adolesc Dev 1979:43-58. https://doi.org/10/fjxcqx.

[470] Van Puyvelde M, Vanfleteren P, Loots G, Deschuyffeleer S, Vinck B, Jacquet W, et al. Tonal synchrony in mother-infant interaction based on harmonic and pentatonic series. Infant Behav Dev 2010;33:387-400. https://doi.org/10/cwqzpk.

[471] Davidson L. Songsinging by young and old: A developmental approach to music. In: Aiello LC, Sloboda J, editors. Music. Percept., Oxford, UK: Oxford University Press; 1994, p. 99-130.

[472] Cambouropoulos E. Voice And Stream: Perceptual And Computational Modeling Of Voice Separation. Music Percept 2008;26:75-94. https://doi.org/10/bmdsbr.

[473] Jordan DS. Influence of the diatonic tonal hierarchy at microtonal intervals. Percept Psychophys 1987;41:482-8. https://doi.org/10/dzxkwk.

[474] Hagel S. Ancient Greek Music: A New Technical History. New York: Cambridge University Press; 2009.

[475] Franklin JC. Hearing Greek Microtones. Anc Greek Music Perform Vienna Wien Stud Beih 2005;29:9-50. 
[476] Seredinskaya VA. The development of the inner hearing during the ear-training classes [Развитие внутреннего слуха в классах сольфеджио]. Moscow: Muzgiz; 1962.

[477] Dumbrill R. The Archaeomusicology of the Ancient Near East. Victoria, Canada: Trafford Publishing; 2005.

[478] Bocquet-Appel J-P, Demars P-Y, Noiret L, Dobrowsky D. Estimates of Upper Palaeolithic meta-populations size in Europe from archaeological data. J Archaeol Sci 2005;32:1656-68. https://doi.org/10/cdmkb5.

[479] Mcbrearty S, Brooks AS. The revolution that wasn't: a new interpretation of the origin of modern human behavior. J Hum Evol 2000;39:453-563. https://doi.org/10/dw4fdk.

[480] Levin TC, Suzukei V. Where Rivers and Mountains Sing: Sound, Music, and Nomadism in Tuva and Beyond. Bloomington, IN: Indiana University Press; 2006.

[481] Reznikoff I. Sound resonance in prehistoric times: A study of Paleolithic painted caves and rocks. J Acoust Soc Am 2008;123:3603-3603. https://doi.org/10/fmngs5.

[482] Díaz-Andreu M, García B. Acoustics and Levantine rock art: auditory perceptions in La Valltorta Gorge (Spain). J Archaeol Sci 2012;39:3591-9. https://doi.org/10/gmnfhn.

[483] Devereux P. Ears and Years: Aspects of Acoustics and Intentionality in Antiquity. In: Scarre C, Lawson G, editors. Archaeoacoustics, Cambridge, UK: McDonald Institute for Archaeological Research; 2006, p. 23-30.

[484] Morley I. The Prehistory of Music: Human Evolution, Archaeology, and the Origins of Musicality. Oxford: Oxford University Press; 2013.

[485] Dams L. Palaeolithic lithophones: descriptions and comparisons. Oxf J Archaeol 1985;4:31-46. https://doi.org/10/d8xr5s.

[486] Blake EC. Stone "Tools" as Portable Sound-Producing Objects in Upper Palaeolithic Contexts: The Application of an Experimental Study. University of Cambridge, 2011.

[487] Conard NJ, Malina M, Münzel S. New flutes document the earliest musical tradition in southwestern Germany. Nature 2009;460:737-40. https://doi.org/10/bh2wr6.

[488] Virtala P, Huotilainen M, Partanen E, Fellman V, Tervaniemi M. Newborn infants' auditory system is sensitive to Western music chord categories. Front Psychol 2013;4. https://doi.org/10/ggwt7j.

[489] Hoeschele M. Animal Pitch Perception: Melodies and Harmonies. Comp Cogn Behav Rev 2017;12:5-18. https://doi.org/10/gmnfzd.

[490] Lots IS, Stone L. Perception of musical consonance and dissonance: An outcome of neural synchronization. J R Soc Interface 2008;5:1429-34. https://doi.org/10/d9vp9s.

[491] Oxenham AJ. The Perception of Musical Tones. In: Deutsch D, editor. Psychol. Music, San Diego, CA: Academic Press; 2013, p. 1-33. https://doi.org/10.1016/B978-0-12-381460-9.00001-8.

[492] Kempe DRC. Living Underground: A History of Cave and Cliff Dwelling. London: Herbert Press; 1988.

[493] James SR, Dennell RW, Gilbert AS, Lewis HT, Gowlett JAJ, Lynch TF, et al. Hominid Use of Fire in the Lower and Middle Pleistocene: A Review of the Evidence [and Comments and Replies]. Curr Anthropol 1989;30:126. https://doi.org/10/d9crf3.

[494] Gowlett JAJ. The early settlement of northern Europe: Fire history in the context of climate change and the social brain. Comptes Rendus - Palevol 2006;5:299-310. https://doi.org/10/d9kq2q.

[495] Roebroeks W, Villa P. On the earliest evidence for habitual use of fire in Europe. Proc Natl Acad Sci 2011;108:5209-14. https://doi.org/10/dz3ht6.

[496] D'Errico F, Henshilwood C, Lawson G, Vanhaeren M, Tillier A-M, Soressi M, et al. Archaeological Evidence for the Emergence of Language, Symbolism, and Music - An Alternative Multidisciplinary Perspective. J World Prehistory 2003;17:1-70. https://doi.org/10/b7tq3n.

[497] Higham T, Basell L, Jacobi R, Wood R, Ramsey CB, Conard NJ. Testing models for the beginnings of the Aurignacian and the advent of figurative art and music: The radiocarbon chronology of Geißenklösterle. J Hum Evol 2012;62:664-76. https://doi.org/10/f32kgd.

[498] Powell A, Shennan S, Thomas MG. Late Pleistocene demography and the appearance of modern human behavior. Science 2009;324:1298-301. https://doi.org/10/b9d65f.

[499] Mellars P, French JC. Tenfold Population Increase in Western Europe at the Neandertal-to-Modern Human Transition. Science 2011;333:623-7. https://doi.org/10/frhx2w.

[500] Hassan FA. Demographic archaeology. New York: Academic Press; 1981.

[501] Nikolsky A. "Talking Jew's Harp" and Its Relation to Vowel Harmony as a Paradigm of Formative Influence of Music on Language. In: Masataka N, editor. Orig. Lang. Revisit. Differ. Music Emergence Neurodiversity Autism, Singapore: Springer Nature; 2020, p. 217-322. https://doi.org/10.1007/978-981-15-4250-3_8.

[502] Kolltveit G. Jew's harps in European archaeology. Oxford UK: Archaeopress; 2006.

[503] Vinogradov VA. Typology of vowel harmony in languages of Africa and Eurasia [Типология сингармонических тенденций в языках Африки и Евразии]. In: Zhurinsky AN, editor. Probl. Afr. Linguist. Typology Comp. Anal. Descr. Lang. Проблемы Африканского Языкознания Типология Компаративистика Описание Языков, Moscow: Nauka; 1972, p. 125-63.

[504] Vinogradov VA. Vowel harmony and word phonology [Сингармонизм и фонология слова]. In: Yunusaliyev B, Yudakhin K, editors. Turkological Res. Тюркологические Исследования, Frunze, the USSR: Ilim; 1970, p. 106-17.

[505] Abayev NV. Chan-Buddhism and cultural-psychological traditions in medieval China [Чань-буддизм и культурно-психологические традиции в средневековом Китаe]. Novosibirsk, Russia: Nauka; 1989.

[506] Simonton DK, Ting S-S. Creativity in Eastern and Western Civilizations: The Lessons of Historiometry. Manag 
Organ Rev 2010;6:329-50. https://doi.org/10/dzqvjw.

[507] Nisbett R. The Geography of Thought: How Asians and Westerners Think Differently...and. New York, London: Simon and Schuster; 2010.

[508] Morris MW, Leung K. Creativity East and West: Perspectives and Parallels. Manag Organ Rev 2010;6:31327. https://doi.org/10/b4gtrn.

[509] Hall DL, Ames RT. Anticipating China: Thinking through the Narratives of Chinese and Western Culture. Albany, NY: State University of New York Press; 1995.

[510] Thrasher AR. The Sociology of Chinese Music: An Introduction. Asian Music 1981;12:17-53. https://doi.org/10/b4jsth.

[511] Lee SE. A History of Far Eastern Art. Upper Saddle River, NJ: Prentice-Hall; 1964.

[512] Elkins J. Chinese Landscape Painting as Western Art History. Hong Kong: Hong Kong University Press; 2010.

[513] Vrabel J, O'Connor TH. When in Boston: A Time Line and Almanac. Boston: Northeastern University Press; 2004.

[514] Zemtsovsky I, Kunanbayeva A. Homo Lyricus, or lyrical song in ethnomusicological stratigraphy of "folk culture" [Homo Lyricus, или лирическая песня в этномузыковедческой стратиграфии «фольклорной культуры»]. In: Ivanova TG, editor. Class. Folk. Today Proc. Conf. 90 Years Anniv. BNPutilovs Birthd., SanktPetersburg: Dmitri Bulanin; 2011, p. 199-249.

[515] Berger K. Bach's Cycle, Mozart's Arrow. Los Angeles, CA: University of California Press; 2008.

[516] Saint-Georges C, Chetouani M, Cassel R, Apicella F, Mahdhaoui A, Muratori F, et al. Motherese in interaction: at the cross-road of emotion and cognition? (A systematic review). PloS One 2013;8:e78103. https://doi.org/10/gmnfxr.

[517] Tonna M, Marchesi C, Parmigiani S. The biological origins of rituals: An interdisciplinary perspective. Neurosci Biobehav Rev 2019;98:95-106. https://doi.org/10.1016/j.neubiorev.2018.12.031.

[518] Dissanayake E. Antecedents of the temporal arts in early mother-infant interaction. In: Wallin NL, Merker B, Brown S, editors. Orig. Music, Cambridge, MA: MIT Press; 2000, p. 389-410.

[519] Mercado El, Mantell JT, Pfordresher PQ. Imitating sounds: A cognitive approach to understanding vocal imitation. Comp Cogn Behav Rev 2014;9:1-57. https://doi.org/10/gmnfhp.

[520] Zarate JM, Wood S, Zatorre RJ. Neural networks involved in voluntary and involuntary vocal pitch regulation in experienced singers. Neuropsychologia 2010;48:607-18. https://doi.org/10/dzt2nj.

[521] Tallmadge WH. Folk Organum: A Study of Origins. Am Music 1984;2:47-65. https://doi.org/10/drqvrr.

[522] Lumaca M, Ravignani A, Baggio G. Music Evolution in the Laboratory: Cultural Transmission Meets Neurophysiology. Front Neurosci 2018;12:246. https://doi.org/10/gdf795.

[523] Teplov B. The psychology of musical abilities [Психология музыкальных способностей]. Moscow: Academy of Pedagogical Sciences of Russia; 1947.

[524] Leontyev AN. The Development of mind. Selected works of Aleksei Nikolaevich Leontyev. Kettering, Ohio: Bookmasters, Inc.; 2009.

[525] Zivic PHR, Shifres F, Cecchi GA. Perceptual basis of evolving Western musical styles. Proc Natl Acad Sci U S A 2013;110:10034-8. https://doi.org/10/f44r7w.

[526] Skrebkov S. Intonation and mode [Интонация и лад]. Sov Muzyka 1967:89-94.

[527] During J, During E. De l'espace lisse au temps troué: à propos des musiques nomades. In: Criton $P$, Chouvel J-M, editors. Deleuze Musique, Paris: Centre de documentation de la musique contemporaine (CDMC); 2015, p. 327-30.

[528] Kushnaryov C. Matters of history and theory of Armenian monodic music [Вопросы истории и теории армянской монодической музыки]. Moscow: Gos Muz Izdat [State Musical Publishing]; 1958.

[529] Alekseyev EYe. Speaking and Singing: Prolegomena to Anthropophonics [Пение и говорение. Основы антропофоники]. Moscow: The Institute of History of Arts at the Ministry of Culture of the Russian Federation; 1993.

[530] Lindestad P-Å, Södersten M, Merker B, Granqvist S. Voice Source Characteristics in Mongolian "Throat Singing" Studied with High-Speed Imaging Technique, Acoustic Spectra, and Inverse Filtering. J Voice 2001;15:78-85. https://doi.org/10/b7gmtq.

[531] Alekseyeva GG. Khomus, play your song for them! [Заиграй им, хомус, свою песню]. In: Grigoryeva A, editor. Music Russ. Music. Creat. Music. Life Repub. Russ. Fed. Музыка России Музыкальное Творчество И Музыкальная Жизнь Республик Российской Федерации, vol. 8, Moscow: Soviet Composer; 1986, p. 321-32.

[532] Sheikin YI. Musical culture of Chukchi [Музыкальная культура чукчей]. Novosibirsk, Russia: Nauka; 2018.

[533] Lawergren B. The origins of musical instruments and sounds. Anthr Int Z Für Völker-U Sprachenkunde 1988;83:31-45.

[534] Alekseyev EYe. The pragmatics, histrionics and rituals in sound imitations of peoples of Northern Siberia [Прагматическое, игровое, ритуальное в звукоподражаниях народов Севера Сибири]. In: Dorokhova E, Zhulanova N, Pashina O, editors. Voice Ritual Proc. Conf. May 1995 Голос И Ритуал Материалы Конференции Май 1995, Moscow: The State Institute of Art Studies at the Ministry of Culture of the Russian Federation; 1995, p. 33-5.

[535] Boersma P, Kovačić G. Spectral characteristics of three styles of Croatian folk singing. J Acoust Soc Am 
2006;119:1805-16. https://doi.org/10/dcxqnw.

[536] Beliaev V. The Formation of folk modal systems. J Int Folk Music Counc 1963;15:4-9. https://doi.org/10/bmtgff.

[537] Beliayev VM. The handbook for measuring folk musical instruments [Руководство для обмера народных музыкальных инструментов]. Moscow: State musical publishing [Госмузиздат]; 1931.

[538] Beliayev VM. On musical folklore and ancient writing systems [О музыкальном фольклоре и древней письменности]. Moscow: Soviet Composer [Советский композитор]; 1971.

[539] Danek G, Hagel S. Homer-Singen. Wien Humanistische BI 1995;37:5-20.

[540] Lawergren B. Mesopotamia, Musical Instruments. Musik Gesch Ggw Allg Enzykl Musik 1997;6:143-71.

[541] Barker A. Text and Sense at Philebus 56A. Class Q 2009;37:103. https://doi.org/10/czdvzp.

[542] Pohlmann E, West ML. Documents of Ancient Greek Music: The Extant Melodies and Fragments. Oxford: Oxford University Press; 2001.

[543] Solomon JD. Towards a history of tonoi. J Musicol 1984;3:242-51.

[544] Gombosi O. Key, Mode, Species. J Am Musicol Soc 1951;4:20-6.

[545] Nikolsky A. Chromatic alteration as expression of aesthetic emotion: from the Ancient doctrine of ethos to the emergence of the notion of musical error. Front Psychol 2016;7. https://doi.org/10/gmnfz3.

[546] Solomon JD. Ptolemy Harmonics: Translation and Commentary. Leiden, The Netherlands: Brill Academic Pub; 2000.

[547] Kholopov Y. Musical-theoretic systems [Музыкально-теоретические системы]. Moscow: Kompozitor; 2006.

[548] Kvitka KV. Selected Works [Избранные труды]. vol. 2. Moscow: Soviet Composer; 1973.

[549] Mathiesen TJ. Greek music theory. In: Christensen T, editor. Camb. Hist. West. Music, Cambridge, UK: Cambridge University Press; 2008, p. 109-35.

[550] Rubtsov F. The foundations of modal morphology of Russian traditional songs [Основы ладового строения русских народных песен]. In: Dunayevskii MA, editor. Essays Music. Folk. Статьи По Музыкальному Фольклору, Moscow: Soviet Composer; 1973, p. 8-81.

[551] Hyer B. Tonality. In: Christensen T, editor. Camb. Hist. West. Music Theory, Cambridge, UK: Cambridge University Press; 2008, p. 726-52.

[552] Hyer B. Key. New Grove Dict Music Music 2001. https://doi.org/10.1093/gmo/9781561592630.article.50818.

[553] Thompson WF. Tonality in music: a general theory. San Marino, CA: Everett Books; 1999.

[554] Nikolsky A. Hemiolic musical mode as post-Hellenistic development of the Ancient Greek chromatic system. Front Psychol 2016;7. https://doi.org/10/gmnfz5.

[555] Kuttner FA. A Musicological Interpretation of the Twelve Lüs in China's Traditional Tone System. Ethnomusicology 1965;9:22-38. https://doi.org/10/bbt6dc.

[556] Marcetteau M. A Queen's Orchestra at the Court of Mari: New Perspectives on the Archaic Instrumentarium in the Third Millennium. In: Dumbrill R, Finkel I, editors. Proc. Int. Conf. East. Archaeomusicology ICONEA Br. Mus. Dec. 4-6 2008, London: Iconea Publications; 2008, p. 67-75.

[557] Krispijn T. Musical Ensembles in Ancient Mesopotamia. In: Dumbrill R, Finkel I, editors. Proc. Int. Conf. East. Archaeomusicology Held Br. Mus. Dec. 4-6 2008, London: Iconea Publications; 2010, p. 125-50.

[558] Rowan D. The Universal Lyre: Three Perspectives. Am Harp J 2013;24:55-63.

[559] Hilprecht H. The Babylonian Expedition of the University of Philadelphia. vol. 20, part 1. Philadelphia: University of Pennsylvania, the Department of Archaeology; 1906.

[560] West ML. The Babylonian musical notation and the Hurrian melodic texts. Music Lett 1994;75:161-79. https://doi.org/10/fb8jwk.

[561] Löhnert A. Manipulating the Gods: Lamenting in Context. In: Radner K, Robson E, editors. Oxf. Handb. Cuneif. Cult., Oxford: Oxford University Press; 2011, p. 402-17.

[562] Duchesne-Guillemin M. Music in Ancient Mesopotamia and Egypt. World Archaeol 1981;12:287-97. https://doi.org/10/fr5n66.

[563] Mirelman S. New developments in the social history of music and musicians in Ancient Iraq, Syria, and Turkey. Music Patronage, Burlington, VT: Ashgate; 2012, p. 3-14.

[564] Ziegler N. Les musiciens et la musique d'après les archives de Mari. Antony, France: Societé pour l'Etude du Proche-Orient Ancient (SEPOA); 2007.

[565] Bryce T. Life and society in the Hittite world. Oxford UK: Oxford University Press; 2004.

[566] Klein J. Three Šulgi Hymns: Sumerian Royal Hymns Glorifying King Šulgi of Ur. Bar-llan University Press; 1981.

[567] Michalowski P. Love or Death? Observations on the Role of the Gala in Ur III Ceremonial Life. J Cuneif Stud 2006;58:49-61. https://doi.org/10/gmnfxc.

[568] Ziegler N. Music: the work of professionals. In: Radner K, Robson E, editors. Oxf. Handb. Cuneif. Cult., Oxford: Oxford University Press; 2011, p. 288-312.

[569] Crickmore L. The tonal systems of Mesopotamia and Ancient Greece: Some similarities and differences. In: Dumbrill R, Marcetteau M, editors. Archaeomusicological Rev. Anc. East, vol. 1, London: 2009, p. 1-16.

[570] Landels JG. Music in Ancient Greece and Rome. London: Routledge; 2002.

[571] Wellesz E. A History of Byzantine Music and Hymnography. Oxford, UK: Clarendon Press; 1961.

[572] Gertsman YV. Byzantine musicology [Византийское музыкознание]. Leningrad: Muzyka; 1988.

[573] Gertsman YV. Ancient music cognition [Античное музыкальное мышление]. Leningrad: Muzyka; 1986. 
[574] Lawergren B. Harps on the Ancient Silk Road. In: Agnew N, editor. Conserv. Anc. Sites Silk Road, Los Angeles, CA: The Getty Conservation Institute; 2010, p. 117-24.

[575] Barinova E. Ethnocultural contacts of China with ethnicities of Central Asia in Antiquity and Middle Ages [Этнокультурные контакты Китая с народами Центральной Азии в древности и средневековье]. Moscow: Russian Academy of Science; 2013.

[576] Mathiesen TJ. Harmonia and Ethos in Ancient Greek Music. J Musicol 1984;3:264-79.

[577] Crickmore L. A possible Mesopotamian origin for Plato's World Soul. Hermathena 2009;186:5-23.

[578] Friberg J. Seven-sided star figures and tuning algorithms in Mesopotamian, Greek, and Islamic texts. Arch Für Orientforsch 2011;52:121-55.

[579] Franklin JC. Harmony in Greek and Indo-Iranian cosmology. J Indo-Eur Stud 2002;30:1-25.

[580] James J. The Music of the Spheres: Music, Science, and the Natural Order of the Universe. New York: Copernicus; 1995.

[581] Barbera A. Arithmetic and geometric divisions of the tetrachord. J Music Theory 1977;21:294-323. https://doi.org/10/bg22vj.

[582] McCartin JB. Mysteries of the Equilateral Triangle. Ruse, Bulgaria: Hikari Ltd; 2010.

[583] Crickmore L. The musicality of Plato. Hermathena 2006:19-43.

[584] Manuel P, Blum S. Classical aesthetic traditions of India, China, and the Middle East. In: Gracyk T, Kania A, editors. Routledge Companion Philos. Music, Abingdon, Oxfordshire: Routledge; 2011, p. 245-56.

[585] Katz J. Music and Aesthetics: An Early Indian Perspective. Early Music 1996;24:407-20.

[586] Thrasher AR. Sizhu instrumental music of south China: Ethos, theory and practice. vol. 84. Leiden, Boston: Brill; 2008.

[587] Henderson JB. The Development and Decline of Chinese Cosmology. Taipei, Taiwan: Windstone Press; 2011.

[588] Meier B. Rhetorical Aspects of the Renaissance Modes. J R Music Assoc 1990;115:182-90. https://doi.org/10/bfkh4f.

[589] Pacholczyk J. Music and Astronomy in the Muslim World. Leonardo 1996;29:145-50. https://doi.org/10/b5wng9.

[590] Yü Y. Trade and expansion in Han China: a study in the structure of Sino-barbarian economic relations. Berkeley, CA: University of California Press; 1967.

[591] Rocconi E. Sounds of war, sounds of peace: for an ethnographic survey of ancient Greek music in Platonic writings. In: Eichmann R, Hickmann E, editors. Music. Perceptions - Past and Present: On Ethnographic Analogy in Music Archeology, Leidorf: Rahden; 2010, p. 117-29.

[592] Roberts JT. The Plague of War: Athens, Sparta, and the Struggle for Ancient Greece. Oxford; New York: Oxford University Press; 2017.

[593] Street J. Music and Politics. Cambridge, UK: John Wiley \& Sons; 2013.

[594] Shestakov VP. From Ethos to Affect. History of musical aesthetics from Antiquity to the 18th century [OT Этоса к Афрфекту. История музыкальной эстетики от античности до XVIII века]. Moscow: Muzyka; 1975.

[595] Bohlman PV. The music of European nationalism: cultural identity and modern history. Santa Barbara, CA: ABCE-CLIO; 2004.

[596] Cao Y, Enke B, Falk A, Giuliano P, Nunn N. Herding, Warfare, and a Culture of Honor: Global Evidence. National Bureau of Economic Research; 2021. https://doi.org/10.3386/w29250.

[597] Linquist S. Which evolutionary model best explains the culture of honour? Biol Philos 2016;31:213-35. https://doi.org/10/f3rjf2.

[598] Orton D. Both subject and object: herding, inalienability and sentient property in prehistory. World Archaeol 2010;42:188-200. https://doi.org/10/bvqpd2.

[599] Pinker S. Enlightenment Now: The Case for Reason, Science, Humanism, and Progress. Illustrated edition. New York, NY: Viking; 2018.

[600] Gadzhiyeva NE. Turkic languages [Тюркские языки]. In: Tenishev ER, editor. Lang. World Turk. Lang. Языки Мира Тюркские Языки, Bishkek, Kyrgyzstan: Russian Academy of Science, Kyrkyzstan; 1997, p. 1734.

[601] Boeckx C, Benítez-Burraco A. The shape of the human language-ready brain. Front Psychol 2014;5:282. https://doi.org/10/gbfwzm.

[602] Mellars P. Symbolism, language, and the Neanderthal mind. In: Mellars P, Gibson KR, editors. Model. Early Hum. Mind, Cambridge, UK: McDonald Institute for Archaeological Research; 1996, p. 15-32.

[603] Benítez-Burraco A, Lattanzi W, Murphy E. Language impairments in ASD resulting from a failed domestication of the human brain. Front Neurosci 2016;10:373. https://doi.org/10/gmnfzq.

[604] Niego A, Benítez-Burraco A. Williams Syndrome, Human Self-Domestication, and Language Evolution. Front Psychol 2019;10:521. https://doi.org/10/gngt5k.

[605] Masataka N. Neurodiversity, giftedness, and aesthetic perceptual judgment of music in children with autism. Front Psychol 2017;8:1595. https://doi.org/10/gbxtvh.

[606] Deruelle C, Schön D, Rondan C, Mancini J. Global and local music perception in children with Williams syndrome. Neuroreport 2005;16:631-4. https://doi.org/10/cpqvrs.

[607] Kitamura Y, Kita Y, Okumura Y, Kaga Y, Okuzumi H, Ishikawa Y, et al. Discrepancy between musical ability and language skills in children with Williams syndrome. Brain Dev 2020;42:248-55. https://doi.org/10/f8qh. 
[608] Thakur D, Martens MA, Smith DS, Roth E. Williams Syndrome and Music: A Systematic Integrative Review. Front Psychol 2018;9:2203. https://doi.org/10/gngt56.

[609] Weiss MW, Sharda M, Lense M, Hyde KL, Trehub SE. Enhanced Memory for Vocal Melodies in Autism Spectrum Disorder and Williams Syndrome. Autism Res Off J Int Soc Autism Res 2021;14:1127-33. https://doi.org/10/gngt6c.

[610] Günther W, Müller N, Trapp W, Haag C, Putz A, Straube A. Quantitative EEG analysis during motor function and music perception in Tourette's syndrome. Eur Arch Psychiatry Clin Neurosci 1996;246:197-202. https://doi.org/10.1007/BF02188953.

[611] Bodeck S, Lappe C, Evers S. Tic-reducing effects of music in patients with Tourette's syndrome: Selfreported and objective analysis. J Neurol Sci 2015;352:41-7. https://doi.org/10/f27mhw.

[612] Amaya V, Descovich K, Paterson MBA, Phillips CJC. Effects of Music Pitch and Tempo on the Behaviour of Kennelled Dogs. Animals 2021;11:10. https://doi.org/10/gngt6f.

[613] Rothenberg D, Roeske TC, Voss HU, Naguib M, Tchernichovski O. Investigation of musicality in birdsong. Hear Res 2014;308:71-83. https://doi.org/10.1016/j.heares.2013.08.016.

[614] McDermott JH, Schultz AF, Undurraga EA, Godoy RA. Indifference to dissonance in native Amazonians reveals cultural variation in music perception. Nature 2016;535:547-50. https://doi.org/10/bmk3.

[615] Arom S, Fernando-Marandola N, Marandola F. An Innovative Method for the Study of African Musical Scales: Cognitive and Technical Aspects. In: Spyridis C, Georgaki A, Kouroupetroglou G, Anagnostopoulou C, editors. Proc. 4th Sound Music Comput. Conf. Lefkada Greece, Athens, Greece: University of Athens; 2007, p. 107-16.

[616] Moelants D, Cornelis O, Leman M. Exploring African tone scales. In: Hirata K, Tzanetakis G, Yoshii K, editors. Proc 10th Int. Soc. Music Inf. Retr. Conf. ISMIR, Kobe, Japan: 2009, p. 489-94.

[617] Luria AR. On changeability of psychological functions in the process of child's development [Об изменчивости психических функций в процессе развития ребенка]. Vopr Psychol 1962;3:15-22. 